\title{
Recommended nuclear data for medical radioisotope production: diagnostic positron emitters
}

\author{
F. T. Tárkányi ${ }^{1} \cdot$ A. V. Ignatyuk ${ }^{2} \cdot$ A. Hermanne ${ }^{3} \cdot$ R. Capote $^{4}$ (i) $\cdot$ B. V. Carlson ${ }^{5} \cdot$ J. W. Engle ${ }^{6} \cdot$ M. A. Kellett $^{7}$.

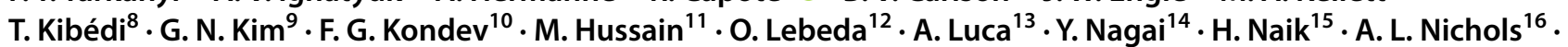 \\ F. M. Nortier $^{6} \cdot$ S. V. Suryanarayana ${ }^{15} \cdot$ S. Takács $^{1} \cdot$ M. Verpelli $^{4}$
}

Received: 16 August 2018 / Published online: 7 January 2019

(c) The Author(s) 2019

\begin{abstract}
An IAEA coordinated research project that began in 2012 and ended in 2016 was primarily dedicated to the compilation, evaluation and recommendation of cross-section data for the production of medical radionuclides. One significant part of this work focused on diagnostic positron emitters. These particular studies consist of 69 reactions for direct and indirect or generator production of ${ }^{44} \mathrm{Sc}\left({ }^{44} \mathrm{Ti}\right),{ }^{52 \mathrm{~m}} \mathrm{Mn}\left({ }^{52} \mathrm{Fe}\right),{ }^{52 \mathrm{~g}} \mathrm{Mn},{ }^{55} \mathrm{Co},{ }^{61} \mathrm{Cu},{ }^{62} \mathrm{Cu}\left({ }^{62} \mathrm{Zn}\right),{ }^{66} \mathrm{Ga},{ }^{68} \mathrm{Ga}\left({ }^{68} \mathrm{Ge}\right),{ }^{72} \mathrm{As}\left({ }^{72} \mathrm{Se}\right),{ }^{73} \mathrm{Se},{ }^{76} \mathrm{Br}$, ${ }^{82} \mathrm{Rb}\left({ }^{82} \mathrm{Sr}\right),{ }^{82 \mathrm{~m}} \mathrm{Rb},{ }^{86} \mathrm{Y},{ }^{89} \mathrm{Zr},{ }^{90} \mathrm{Nb},{ }^{94 \mathrm{~m}} \mathrm{Tc},{ }^{110 \mathrm{~m}} \mathrm{In}\left({ }^{110} \mathrm{Sn}\right),{ }^{118} \mathrm{Sb}\left({ }^{118} \mathrm{Te}\right),{ }^{120} \mathrm{I},{ }^{122} \mathrm{I}\left({ }^{122} \mathrm{Xe}\right),{ }^{128} \mathrm{Cs}\left({ }^{128} \mathrm{Ba}\right)$, and ${ }^{140} \mathrm{Pr}\left({ }^{140} \mathrm{Nd}\right)$ medical radionuclides. The resulting reference cross-section data were obtained from Padé fits to selected and corrected experimental data, and integral thick target yields were subsequently deduced. Uncertainties in the fitted results were estimated via a Padé least-squares method with the addition of a $4 \%$ assessed systematic uncertainty. Experimental data were also compared with theoretical predictions available from the TENDL-2015 and TENDL-2017 libraries. All of the numerical reference cross-section data with their corresponding uncertainties and deduced integral thick target yields are available on-line at the IAEA-NDS medical portal www-nds.iaea.org/medicalportal and also at the IAEA-NDS web page www-nds.iaea.org/ medical/positron_emitters.html.
\end{abstract}

Keywords IAEA coordinated research project - Diagnostic medical isotopes · Positron emitters · Cross-section evaluation · Uncertainty estimation $\cdot$ Padé fit $\cdot$ Bayesian inference $\cdot$ Recommended $\sigma$ and yield data

R. Capote

r.capotenoy@iaea.org

1 Institute for Nuclear Research, Hungarian Academy of Sciences, Debrecen, Hungary

2 Institute of Physics and Power Engineering (IPPE), Obninsk, Russia

3 Vrije Universiteit Brussel, Brussels, Belgium

4 NAPC, Nuclear Data Section, International Atomic Energy Agency, 1400 Vienna, Austria

5 Instituto Tecnológico de Aeronáutica, São José dos Campos, Brazil

6 Los Alamos National Laboratory (LANL), Los Alamos, USA

7 Laboratoire National Henri Becquerel (LNHB), CEA Saclay, Paris, France
8 Australian National University (ANU), Canberra, Australia

9 Kyungpook National University, Daegu, Republic of Korea

10 Argonne National Laboratory (ANL), Lemont, USA

11 Government College University, Lahore, Pakistan

12 Nuclear Physics Institute, Rez, Prague, Czech Republic

13 National Institute of Physics and Nuclear Engineering "Horia Hulubei”, Măgurele, Romania

14 Japan Atomic Energy Agency (JAEA), Tokaimura Naka, Ibaraki-ken, Japan

15 Bhabha Atomic Research Centre (BARC), Trombay, Mumbai, India

16 University of Surrey, Guildford, UK 


\section{Introduction}

The importance of positron-emitting radionuclides in molecular imaging (Positron Emission Tomography, PET) has constantly increased over the years, especially to follow metabolic processes and to quantify radiation dose in internal radiotherapy. Nuclear data identified with these radionuclides are important for the optimisation of their production routes and medical applications. Judicious selection of the projectile energy range will maximise the yield of the product and minimise that of any radioactive impurities. Several charged-particle and neutron production routes exist for the production of such radionuclides. The International Atomic Energy Agency (IAEA) initiated and supported a Coordinated Research Project (CRP) from 1997 to 2001 with the primary aim of establishing a reference nuclear reaction database for the most important gamma-ray and positron emitters and associated monitor reactions in order to optimise their production [1]. No uncertainties in the recommended data were produced at that time. The list of positron emitters that was included in this earlier effort is shown in Table 1. The reference cross-section data and integral thick target yields were made available in a hard-copy technical document, and later became accessible on the medical portal of the IAEA Nuclear Data Section (IAEA-NDS) with further updates from 2001 to 2007 [2].

Over the previous two decades new experimental data have been measured for the earlier evaluated reactions, and numerous new and potentially suitable candidate PET-isotopes have appeared in the literature along with pre-clinical studies. Therefore, a new CRP was initiated at the end of 2012 in order to redefine the production routes and upgrade the production data for the previously studied radionuclides, and to complement the database with the equivalent results for emerging prospective radionuclides. An additional goal of the working programme was to provide uncertainties for the recommended cross sections of all the reactions studied [3]. The results of this evaluation work are summarised for production routes applicable to diagnostic positron emitters, including generator systems for short-lived radionuclides.

\section{Evaluation method}

The evaluation process was performed in a similar manner to previous studies [1, 6], and includes the following steps that will be discussed in more detail below:

- thorough compilation of experimental data (Section "Thorough compilation of experimental data");

- undertake new measurements if required (Section "New CRP measurements");

- correct and normalise earlier experimental data (decay data, enriched target abundances, monitor data, recognised systematic errors) (Sections "Status of earlier experimental data" and "Correction of earlier experimental data");

- compare with theoretical predictions (Section "Comparisons with theoretical predictions");

- critical comparison of all experimental datasets, and rejection of unreliable and erroneous sets (Section "Critical comparisons and selection of the most reliable experimental data");

- least-square fit of selected experimental datasets to derive mean values and corresponding uncertainties of the resulting recommended reference data (Section "Data fitting and resulting uncertainties- Padé fit of selected experimental data");

- calculate integral yield as a function of the incident particle energy (Section "Integral yields for thick targets as a function of particle energy").

Table 1 Earlier evaluated nuclear reactions for the production of diagnostic PET isotopes (1995-1999), and also made available as an IAEA nuclear database $[1,2]$

\begin{tabular}{|c|c|c|c|c|c|c|c|}
\hline $\begin{array}{l}\text { PET radionu- } \\
\text { clide }\end{array}$ & Half-life & Decay $(\%)$ & $\begin{array}{l}\text { End-point energy } \\
(\mathrm{keV})\end{array}$ & $\begin{array}{l}\text { Reaction } \\
\text { product }\end{array}$ & Half-life & Decay $(\%)$ & Production reaction \\
\hline${ }^{11} \mathrm{C}$ & $1221.8 \mathrm{~s}$ & $99.7669 \beta^{+}$ & 960.4 & ${ }^{11} \mathrm{C}$ & $1221.8 \mathrm{~s}$ & $99.7669 \beta^{+}$ & ${ }^{14} \mathrm{~N}(\mathrm{p}, \boldsymbol{\alpha}){ }^{11} \mathrm{C}$ \\
\hline${ }^{13} \mathrm{~N}$ & $9.965 \mathrm{~min}$ & $99.8036 \beta^{+}$ & 1198.5 & ${ }^{13} \mathrm{~N}$ & $9.965 \mathrm{~min}$ & $99.8036 \beta^{+}$ & ${ }^{16} \mathrm{O}(\mathrm{p}, \alpha){ }^{13} \mathrm{~N}$ \\
\hline${ }^{15} \mathrm{O}$ & $122.24 \mathrm{~s}$ & $99.9003 \beta^{+}$ & 1732.0 & ${ }^{15} \mathrm{O}$ & $122.24 \mathrm{~s}$ & $99.9003 \beta^{+}$ & $\begin{array}{l}{ }^{15} \mathrm{~N}(\mathrm{p}, \mathrm{n}){ }^{15} \mathrm{O} \\
{ }^{14} \mathrm{~N}(\mathrm{~d}, \mathrm{n}){ }^{15} \mathrm{O}\end{array}$ \\
\hline${ }^{18} \mathrm{~F}$ & $109.77 \mathrm{~min}$ & $96.73 \beta^{+}$ & 633.5 & ${ }^{18} \mathrm{~F}$ & $109.77 \mathrm{~min}$ & $96.73 \beta^{+}$ & $\begin{array}{l}{ }^{18} \mathrm{O}(\mathrm{p}, \mathrm{n}){ }^{18} \mathrm{~F} \\
{ }^{\mathrm{nat}} \mathrm{Ne}(\mathrm{d}, \mathrm{x}){ }^{18} \mathrm{~F}\end{array}$ \\
\hline${ }^{68} \mathrm{Ga}$ & $67.71 \mathrm{~min}$ & $88.91 \beta^{+}$ & 1899.1 & ${ }^{68} \mathrm{Ge}$ & $270.95 \mathrm{~d}$ & $100 \mathrm{EC}$ & $\begin{array}{l}{ }^{69} \mathrm{Ga}(\mathrm{p}, 2 \mathrm{n}){ }^{68} \mathrm{Ge} \\
{ }^{\text {nat }} \mathrm{Ga}(\mathrm{p}, \mathrm{xn}){ }^{68} \mathrm{Ge}\end{array}$ \\
\hline${ }^{82} \mathrm{Rb}$ & $1.2575 \mathrm{~min}$ & $95.43 \beta^{+}$ & 3378 & ${ }^{82} \mathrm{Sr}$ & $25.35 \mathrm{~d}$ & $100 \mathrm{EC}$ & $\begin{array}{l}{ }^{85} \mathrm{Rb}(\mathrm{p}, 4 \mathrm{n}){ }^{82} \mathrm{Sr} \\
{ }^{\mathrm{nat}} \mathrm{Rb}(\mathrm{p}, \mathrm{xn}){ }^{82} \mathrm{Sr}\end{array}$ \\
\hline
\end{tabular}

Decay data as tabulated have been taken from NuDat (NuDat 2.6 or 2.7), a user-friendly form of ENSDF [4, 5] 


\section{Thorough compilation of experimental data}

Detailed searches for published experimental cross sections were made, including the following sources: primary publications in journals, EXFOR database of IAEA-NDS [7], IAEA INIS database [8], evaluated libraries (ENDF) [9], bibliographies of Brookhaven National Laboratory (Burrows and Dempsey [10], Holden et al. [11], Karlstrom and Christman [12]), reports of the International Atomic Energy Agency (Dmitriev [13], Gandarias-Cruz and Okamoto [14]), Landolt Börnstein Series [15], Landolt Börnstein New Series [16], Tobailem et al. [17], Albert et al. [18], Münzel et al. [19], PhD theses, other relevant evaluations, private communications, etc. All experimental references are cited in each of the subsections that describe specific reaction paths.

The cut-off for inclusion of new data was set at June 2016, and therefore some results published in the final year of this compilation exercise were not added into the already completed fits but are still shown among the datasets retrieved. Duplications of original data published in the numerous review papers on production and/or use of medical radionuclides are explicitly mentioned in this study.

\section{New CRP measurements}

Additional experiments were performed by various CRP participants as crucial support in defining the excitation functions of the ${ }^{45} \mathrm{Sc}(\mathrm{d}, 3 \mathrm{n}){ }^{44} \mathrm{Ti},{ }^{\text {nat }} \mathrm{Ni}(\mathrm{p}, \mathrm{x}){ }^{52} \mathrm{Fe},{ }^{55} \mathrm{Mn}(\mathrm{p}, 4 \mathrm{n}){ }^{52} \mathrm{Fe}$, ${ }^{50} \mathrm{Cr}(\alpha, 2 \mathrm{n}){ }^{52} \mathrm{Fe},{ }^{58} \mathrm{Ni}(\mathrm{p}, \alpha){ }^{55} \mathrm{Co},{ }^{61} \mathrm{Ni}(\mathrm{p}, \mathrm{n}){ }^{61} \mathrm{Cu},{ }^{60} \mathrm{Ni}(\mathrm{d}, \mathrm{n}){ }^{61} \mathrm{Cu}$, ${ }^{n a t} \mathrm{Ga}(\mathrm{p}, \mathrm{x}){ }^{68} \mathrm{Ge},{ }^{\text {nat }} \mathrm{Ge}(\mathrm{p}, \mathrm{xn}){ }^{72} \mathrm{As}$, ${ }^{\text {nat }} \mathrm{Ge}(\mathrm{d}, \mathrm{xn}){ }^{72} \mathrm{As}$, ${ }^{89} \mathrm{Y}(\mathrm{d}, 2 \mathrm{n}){ }^{89} \mathrm{Zr},{ }^{93} \mathrm{Nb}(\mathrm{p}, \mathrm{x}){ }^{90} \mathrm{Nb},{ }^{92} \mathrm{Mo}(\alpha, \mathrm{x}){ }^{94 \mathrm{~m}} \mathrm{Tc}$, ${ }^{110} \mathrm{Cd}(\mathrm{p}, \mathrm{n}){ }^{110 \mathrm{~m}} \mathrm{In},{ }^{107} \mathrm{Ag}(\alpha, \mathrm{n}){ }^{110 \mathrm{~m}} \mathrm{In},{ }^{\text {nat }} \mathrm{Sb}(\mathrm{p}, \mathrm{xn}){ }^{1{ }^{18}} \mathrm{Te}$ and ${ }^{141} \operatorname{Pr}(\mathrm{d}, 3 \mathrm{n}){ }^{140} \mathrm{Nd}$ reactions. Details of these studies have been reported individually and separately in other dedicated publications (see references for specific reactions given below).

\section{Status of earlier experimental data}

Large sets of experimental data are available for some reactions, but only one or two relevant measurements exist for other reactions. Investigation of the published data permits some general remarks and conclusions to be made:

- Early investigations from 1945 to 1970 were mostly dedicated to the study of nuclear reaction mechanisms, and were performed on accelerators possessing somewhat limited technology of that time. The information on decay data and estimated uncertainties adopted for these experiments are poor in most cases.

- Production of medical radionuclides used in clinical practice would normally involve monoisotopic or at least enriched targets. However, production cross sections are sometimes determined by evaluators from data obtained with natural targets over limited energy ranges (up to the threshold of the next contributing reaction) in order to derive suitable data for subsequent evaluation. These data are in many cases more reliable due to the higher quality of the targets employed (with respect to thickness and uniformity).

- Nowadays, excitation functions are commonly measured over a broader energy range by means of the stacked-foil technique. This method possesses significant advantages in irradiation time and the determination of good relative values because of the fixed number of bombarding particles in each sample. However, long stacks suffer from an accumulation of effects caused by uncertainties in foil thicknesses that result in a possible increasing energy shift throughout the stack. The precise energy in each foil can be controlled by simultaneous measurements of the excitation function of reference monitor reactions over the whole energy range, but this is very rarely undertaken.

- Another possibility is to irradiate a large number of targets simultaneously in conjunction with rotating wheels in which different energy absorbers with well-measured thicknesses are inserted before each target. The number of bombarding particles incident on each target is the same and well controlled, although one disadvantage is the much longer irradiation time needed when compared with stacked-foil irradiations.

- Overwhelming parts of the datasets exhibit consistent and realistic behaviour, but in some cases points in a given set may disagree significantly from the observed trend and with other data without any obvious reason (although a most probable cause is an incorrect estimation of the real target thickness). These clearly discrepant data points were not considered as valid data during the fitting process.

Knowledge of the energy and energy distribution of the incident particle beam is important in reducing the uncertainty of the energy values of the data points. This information is preferably obtained by prior measurement, However, these incident beam parameters are rarely known for production machines in which the use of high-intensity beams causes changes in target quality (i.e., surface density and uniformity of target atoms). Gas targets are especially sensitive to density reduction caused by the heat generated from high-current beams.

Essentially two methods were used in these experiments to determine the number of incident particles: direct collection of the total charge in a Faraday cup, or indirectly by means of the reference data from a series of monitor reactions. Some experiments involve only the activity of a single 
monitor foil inserted in front of the target stack compared with the activity of the same foil target measured in a Faraday cup at the same energy. An additional factor of uncertainty is the constancy over time of the number of incident particles, especially when the half-life of the radionuclide investigated is comparable to or shorter than the irradiation time. Not all laboratories have the instrumentation needed to monitor and quantify the beam intensity on the target during the irradiation.

Other factors are the method of detection of the different types of radiation emitted in order to quantify the product nuclei: X-rays, gamma rays, alpha and beta particles, and neutron emissions involve the use of detectors that possess very different energy resolution and efficiency. Nevertheless, recent developments in detector technology have resulted in greater reliability and commensurate reductions in the uncertainties. Compilations and evaluations of the measured results and assessment of the quality of the reported work from different laboratories require all these factors be taken into account, which requires detailed investigations of all of the original publications.

\section{Correction of earlier experimental data}

Where possible, published cross sections that rely on outdated decay data were corrected by taking new decay data into account by means of NuDat [4] (with the Evaluated Nuclear Structure Data File (ENSDF) [5] as the data source). This form of correction was also carried out with respect to the decay data associated with the adopted monitor reactions. As any correction with respect to updated half lives has a non-linear impact on the well-known activation equation used to determine the cross section (primarily factors related to time), caution has to be taken when applying such adjustments. They are only possible if timing information is available in the original publication. The correction for other linear factors can be more easily performed, but also requires knowledge of the decay data used by the original authors.

Excitation functions measured over a broad energy range that show often relatively small uncertainties are sometimes significantly different from more reliable data determined over a shorter energy range. These higher energy data were normalised in such cases to the well measured data to produce reference data in a broad energy range. The same method was also adopted in the case of systematic energy shifts observed in the stacked-foil technique, which can be linearly corrected with respect to data measured on accelerators with high-energy definition.

Fitting procedures require reliable uncertainties in the experimental data selected for such statistical analyses. Unfortunately, the uncertainties in the cross-section values and the beam energy are not always appropriately provided as a significant part of the published experimental data.
Therefore, missing cross-section uncertainties were estimated on the basis of the measurement methodology and the experience of the compilers/evaluators.

The experimental data for a given reaction measured with similar methods and comparable technology in different laboratories often have significantly different quoted uncertainties. Some studies report uncertainties that are unrealistically low because all contributing statistical or systematic effects have not been taken into account, or are incorrectly estimated. Therefore, such values were corrected to more realistic average uncertainties to avoid incorrect weighting in the fitting procedure.

\section{Comparisons with theoretical predictions}

Experimental data were compared with theoretical predictions of activation by charged-particle reactions, as assembled and made available in the online TENDL-2015 and TENDL-2017 libraries [20]. Both of these libraries are based on the reaction modelling adopted in the TALYS code [21]. The TENDL libraries are derived from both default and adjusted TALYS calculations, and occasionally from other sources.

The aim of the comparisons was to obtain a general impression of the shape of each excitation function over a broad energy range, including the magnitude of the maximum cross-section value and the effective threshold of reaction in cases where there were contradicting data. These predictions also permitted extrapolation of the excitation function in cases where experimental data were only available over a short and limited energy range as input for the Bayesian least-square fit (e.g., for the Padé fit). All TENDL predictions are shown along with the experimental data in the various figures of the excitation functions. Recently published results of evaluations for different activation products obtained from fitting by adjustment of theoretical codes to a compilation of existing experimental results (including some from this coordinated research project) have not been considered here, as the Bayesian non-model evaluation is the preferred evaluation method.

\section{Critical comparisons and selection of the most reliable experimental data}

Corrected and analysed experimental data were visually compared in figures that also included the theoretical predictions.

\section{Measurement of radioactivity}

The main sources of error when determining absolute activity are faulty estimates of the detector efficiency (especially in the low-energy region), self-absorption of low-energy 
gamma rays, geometry deviations between point source calibrations and the activated spot size on targets, dead-time and pile-up corrections, and the adoption of incorrect decay data.

\section{Determination of the energy scale}

Errors in the energy scale are introduced by improper estimation of the energy of the primary beam, uncertainties in the effective thickness of the individual targets, the cumulative effect of the stacked-foil technique, and the ill-defined impact of absorbers introduced to vary the energy of the incoming beam. Accelerators used for data measurements in nuclear physics usually have the necessary facilities to measure the energy of the beams precisely.

\section{Estimation of uncertainties of the cross sections and energy scale}

Despite the existence of the JCGM guide for the expression of uncertainty in measurements that experimentalists are strongly advised to follow [22], we have found that no such recommended systematic procedures have been undertaken to estimate properly the uncertainty of the measured cross sections and their energy scale. Various factors contribute to the assessment of the uncertainty associated with crosssection measurements. Unfortunately, authors in many original publications present only the total uncertainty in their tables of cross sections, without discussing or defining the estimated uncertainties of the contributing processes (i.e., no sufficiently detailed uncertainty budget is given).

A number of noteworhy observations were made during the course of the evaluation process:

- data from different authors often show striking systematic disagreements over the whole energy range;

- data below the reaction threshold were frequently reported;

- sometimes the data were extensively scattered, without any explanation;

- specific laboratories carried out systematic investigations, and as a consequence generated good results for many reactions.

Due to a general lack of information reported in the original publications and earlier compilations, the quality of the data could not be assessed in most cases, nor reasons identified for disagreements with other publications apart from a few exceptions. The most likely sources of disagreement or reasons for discrepancies among the experimental data were as follows:

Beam current. While relying on monitor reactions, the main problem originates from the use of outdated monitor cross sections. Another source of error was improper use of monitors, especially an incorrect estimation of the energy of the bombarding particle in a region where the excitation function curve has a steep slope (which will lead to an under- or overestimation of the beam flux).

Determination of the number of target nuclei. Although difficult to determine the number of target nuclei with high precision, an uncertainty below five percent can be easily achieved. The main challenges in the case of thin solid targets are uncertainties associated with the chemical state caused by surface oxidation, non-uniformity in the thickness of the foil, and improper estimation of the shape or dimensions influencing the thickness derived from weighing. Furthermore, well-known density reductions along the beam due to the heat effect play a very important role in the case of gas targets.

On the basis of emerging inconsistencies and trends, contradictory and scattered data were rejected from the analyses. Such an extensive selection process takes into account many factors, of which a few cannot be formulated in a mathematical manner, but rather are based on invaluable experienced, yet subjective, judgements by the evaluators.

\section{Data fitting and resulting uncertainties- Padé fit of selected experimental data}

Previous evaluations of the experimental cross-section data for diagnostic radionuclides were usually fitted by the spline method. Such a procedure is based on a piecemeal approximation of the data between specified points (knots of the spline) based on individual interpolating polynomials. These polynomials match in such a way that the zeroeth, first and second derivatives are continuous at the knots, and are usually selected by the second (quadratic interpolation) or third order (cubic interpolation). A continuous and smooth fit is obtained with minimum twisting (oscillating behaviour) of the fitting curve, which arises from the conditions for continuity. A particular feature of the spline method is that the fit in a selected interval is independent from the data in other intervals.

The spline method is well known (e.g., see Ref. [23] and references therein), and has been applied in nuclear data evaluations. Some known shortcomings relate to the following requirements and inadequacies:

- knots have to be selected by a user, which makes the fit time consuming with partially arbitrary results;

- cubic splines are not always adequate for complex-shaped curves.

A more general class of analytical function is the rational function defined as the ratio of two polynomials. Such an approximation was proposed by Padé over one hundred 
and twenty years ago [24], and has become one of the most important interpolation techniques of statistical mathematics $[25,26]$. As a rational function, the Padé approximant can be expressed by a set of real polynomial coefficients, or by a set of real coefficients of the pole expansion

$p_{L}(z)=c+\sum_{l} \frac{a_{l}}{z-\eta_{l}}+\sum_{k} \frac{\alpha_{k}\left(z-\varepsilon_{k}\right)+\beta_{k}}{\left(z-\varepsilon_{k}\right)^{2}+\gamma_{k}^{2}}$,

where $z=x+i y$ are complex variables, and $L$ is the order of the polynomial representation of the Padé approximant (therefore all coefficients depend implicitly on $L$ ) [25, 26]. This equation is also called the resonance expansion, in which $\varepsilon_{k}$ and $\gamma_{k}$ are the energy and the total half-width of the $k$ th resonance, respectively, while $\alpha_{k}$ and $\beta_{k}$ are the partial widths and interference parameters. The first sum corresponds to the real poles, while the second sum relates to the complex poles.

Effective codes for practical applications of the Padé approximation were developed by the IPPE, Obninsk group [27]. The simplest version of these codes permits analyses of up to 500 experimental points, with the number of parameters $L \leq 40$ and the ratio limit of analysed functions up to $f^{\max } / f^{\min } \leq 10^{6}$. A more detailed description of the method can be found in Ref. [27], and some important questions of application are presented in Refs. [28, 29].

Padé approximations are also very convenient for calculations of the data uncertainties and the corresponding covariance matrices. The fitting procedure is always based on a minimisation of the deviation functional

$\chi^{2}=(N-L)^{-1} \sum_{j=1}^{N}\left(p_{L}\left(x_{j}\right)-f_{j}\right)^{2} / \sigma_{j}^{2}$,

where $f_{j}$ are the available experimental data, $\sigma_{j}$ are their total uncertainties (including both systematic and statistical components) and $N$ is the number of analysed points. Such minimisation is carried out iteratively by means of the discrete optimization approach. Minimal deviation for a given $L$ is computed by assessing and selecting $L$ points from the available $N$ points $(L<N)$, and then determining the corresponding approximants from Eq. (2). Once this process has been completed, $L$ is changed and the iteration is repeated until an overall minimum is found from all discrete possibilities available. Some additional details of the method are considered in our earlier paper that focused on the evaluation of charged-particle monitor reactions [30].

Along with a consistent consideration of the statistical uncertainties of experimental data, the Padé method allows the determination of some systematic uncertainties that are usually underestimated by their authors, and also establishes some implicit correlations of the data. The averaged deviation of the full experimental dataset from the approximating function is regarded as the systematic uncertainty, while the variances of deviations around the averaged values are regarded as the statistical uncertainties. An optimal description of all data is achieved by the traditional iteration procedure of minimizing the mean squares deviations with the statistical and systematic uncertainties.

Only total uncertainties are determined in the majority of the experimental studies, and reasonable reconstructions of the corresponding systematic uncertainties are judged to be impossible to achieve in many of these cases. The method described above provides estimates of the systematic uncertainties on the basis of general statistical criteria which are valid for a reasonable number of studies. However, for a small number of the experimental measurements, underestimation of the systematic uncertainties is highly probable. Such underestimations also occur in those cases whereby the same, very similar, or other components of the same experimental equipment are used in a range of different studies, since any related correlations have been neglected.

After analysing the complete set of available data, we have come to the conclusion that realistic total uncertainties cannot be defined as less than $4 \%$ for each of the reactions considered. Therefore, an additional systematic uncertainty of $4 \%$ has been introduced as part of each systematic uncertainty derived from statistical analyses of all the recommended cross sections.

\section{Integral yields for thick targets as a function of particle energy}

Integral thick target yields as a function of energy were calculated from the recommended cross section data. We have quantified the production rates for radionuclides whose half-lives are short relative to the length of irradiation. This rate is also known as the "physical yield", or "instantaneous production rate", since the effect of decay of the radionuclide is small compared with the activity being created. Two other yields are also defined on the IAEA medical portal [2], namely the activity of a fixed $1 \mathrm{~h}$ and $1 \mu \mathrm{A}$ irradiation, and the saturation activity at EOB for a $1 \mu \mathrm{A}$ irradiation.

The activity of a fixed $1 \mathrm{~h} / 1 \mu \mathrm{A}$ irradiation is meaningful and can be used in practice for longer-lived radionuclides where the activity is increasing linearly with irradiation time. Saturation activity is used in the case of short half-life radionuclides, when a constant activity is obtained for even relatively short irradiations. The definition of these parameters can be found in Bonardi [31], and Otuka and Takács [32]. Thick target yields for the different production routes leading to a given radionuclide are summarised within a figure at the end of every subsection. 
Medically relevant radionuclides can be obtained in many cases from the decay of a parent with a different half-life (indirect production, or generator couple). Two separate figures are shown in such cases, corresponding to the yields at EOB for the shorter-lived daughter and the longer-lived parent (often orders of magnitude lower). Depending on the relative half-lives, either time-dependent partial equilibrium (indirect production in which mother and daughter have comparable half-lives), or total equilibrium (long-lived parent/short-lived daughter generators) is obtained.

\section{Results for charged-particle reactions}

The list of reactions evaluated in the present studies consists altogether of 69 charged-particle reactions for the production of 23 radionuclides of interest for PET imaging, including 11 generator systems for short-lived medically interesting radioisotopes (Table 2). There are 39 proton reactions, 16 deuteron reactions, one reaction for ${ }^{3} \mathrm{He}$, and 13 reactions for $\alpha$ particles. Energies of incident particles cover the range from a few $\mathrm{MeV}$ up to $100 \mathrm{MeV}$. Every subsequent subsection contains a summary of the most frequent use of each of the 23 medically relevant radionuclides, and the literature references found for each production route (given in both the text and figures), selected data (text and figures) and the characteristics of the Padé fit (text and figures). As mentioned previously, the physical yields are included in one or two additional figures at the end of each subsection if indirect and/or generator production is being considered.

Half-lives and limited decay-scheme data for the different radionuclides discussed in the following subsections can be found in Table 2. The $\gamma$-ray energies in $\mathrm{keV}$ and the corresponding absolute emission probabilities (absolute intensities, $P_{\gamma}(\%)$ ) used to identify and quantify the activity of a given radionuclide in the experimental studies (and $\beta^{+}$decay fraction instead of the intensity of the $511 \mathrm{keV}$ annihilation radiation) are listed, and have also been included within each of the primary subsections of this Section.

Reactions for radionuclides present in Table 1 but not considered during the course of this CRP will be evaluated in a similar manner as part of a future series of IAEA-sponsored studies and will be published elsewhere.

Table 2 Evaluated nuclear reactions and decay data adopted for production of diagnostic PET radionuclides (2012-2016) [2]. Reaction thresholds for natural targets estimated approximately from the plots, and are given in bold

\begin{tabular}{|c|c|c|c|c|c|c|c|c|}
\hline $\begin{array}{l}\text { PET } \\
\text { radionu- } \\
\text { clide }\end{array}$ & Target & Reaction & $\begin{array}{l}\text { Reaction } \\
\text { threshold } \\
(\mathrm{MeV})\end{array}$ & $\begin{array}{l}\text { Reaction } \\
\text { product }\end{array}$ & Half-life $^{\mathrm{a}}$ & $\begin{array}{l}\text { Decay }(\%)^{\mathrm{a}} \\
E_{\gamma}(\mathrm{keV}), P_{\gamma}(\%)\end{array}$ & $\begin{array}{l}\text { Order of Padé } \\
\text { polynomial L }\end{array}$ & Comment \\
\hline \multirow[t]{5}{*}{${ }^{44} \mathrm{Sc}$} & ${ }^{44} \mathrm{Ca}$ & ${ }^{44} \mathrm{Ca}(\mathrm{p}, \mathrm{n}){ }^{44} \mathrm{Sc}$ & 4.537 & ${ }^{44} \mathrm{Sc}$ & $3.97 \mathrm{~h}$ & $\mathrm{EC}+\beta^{+} 100\left(\beta^{+} 94.27\right)$ & 19 & Direct \\
\hline & ${ }^{44} \mathrm{Ca}$ & ${ }^{44} \mathrm{Ca}(\mathrm{d}, 2 \mathrm{n}){ }^{44} \mathrm{Sc}$ & 6.965 & & & $\gamma: 1157.020,99.9$ & 9 & Direct \\
\hline & ${ }^{43} \mathrm{Ca}$ & ${ }^{43} \mathrm{Ca}(\mathrm{d}, \mathrm{n}){ }^{44} \mathrm{Sc}$ & 0 & & & & 5 & Direct \\
\hline & ${ }^{45} \mathrm{Sc}$ & ${ }^{45} \mathrm{Sc}(\mathrm{p}, 2 \mathrm{n}){ }^{44} \mathrm{Ti}$ & 12.65 & ${ }^{44} \mathrm{Ti}$ & $59.1 \mathrm{y}$ & EC 100 & 7 & Generator \\
\hline & ${ }^{45} \mathrm{Sc}$ & ${ }^{45} \mathrm{Sc}(\mathrm{d}, 3 \mathrm{n}){ }^{44} \mathrm{Ti}$ & 15.25 & & & From decay of ${ }^{44} \mathrm{Sc}$ & 6 & Generator \\
\hline \multirow[t]{5}{*}{${ }^{52 \mathrm{~m}} \mathrm{Mn}$} & ${ }^{\text {nat }} \mathrm{Ni}$ & ${ }^{\text {nat }} \mathrm{Ni}(\mathrm{p}, \mathrm{x})^{52} \mathrm{Fe}$ & 40 & ${ }^{52} \mathrm{Fe}$ & $8.275 \mathrm{~h}$ & $\mathrm{EC}+\beta^{+} 100\left(\beta^{+} 55.49\right)$ & 11 & Generator \\
\hline & ${ }^{55} \mathrm{Mn}$ & ${ }^{55} \mathrm{Mn}(\mathrm{p}, 4 \mathrm{n}){ }^{52} \mathrm{Fe}$ & 35.00 & & & From decay of ${ }^{52 \mathrm{~m}} \mathrm{Mn}$ & 17 & Generator \\
\hline & ${ }^{50} \mathrm{Cr}$ & ${ }^{50} \mathrm{Cr}(\alpha, 2 \mathrm{n}){ }^{52} \mathrm{Fe}$ & 16.90 & & & & 9 & Generator \\
\hline & ${ }^{52} \mathrm{Cr}$ & ${ }^{52} \mathrm{Cr}(\mathrm{p}, \mathrm{n}){ }^{52 \mathrm{~m}} \mathrm{Mn}$ & 5.60 & ${ }^{52 \mathrm{~m}} \mathrm{Mn}$ & $21.1 \mathrm{~min}$ & $\mathrm{EC}+\beta^{+} 98.22\left(\beta^{+} 96.6\right)$ & 14 & Direct \\
\hline & ${ }^{52} \mathrm{Cr}$ & ${ }^{52} \mathrm{Cr}(\mathrm{d}, 2 \mathrm{n}){ }^{52 \mathrm{~m}} \mathrm{Mn}$ & 8.02 & & & $\begin{array}{l}\text { IT } 1.78 \\
\gamma: 1434.092,98.2\end{array}$ & 8 & Direct \\
\hline \multirow[t]{2}{*}{${ }^{52} \mathrm{Mn}$} & ${ }^{52} \mathrm{Cr}$ & ${ }^{52} \mathrm{Cr}(\mathrm{p}, \mathrm{n}){ }^{52 \mathrm{~g}} \mathrm{Mn}(\mathrm{m}+)$ & 5.60 & ${ }^{52 \mathrm{~g}} \mathrm{Mn}$ & $5.591 \mathrm{~d}$ & $\mathrm{EC}+\beta^{+} 100\left(\beta^{+} 29.4\right)$ & 9 & Direct \\
\hline & ${ }^{52} \mathrm{Cr}$ & ${ }^{52} \mathrm{Cr}(\mathrm{d}, 2 \mathrm{n}){ }^{52 \mathrm{~g}} \mathrm{Mn}(\mathrm{m}+)$ & 8.02 & & & $\begin{array}{l}\gamma: 744.233,90.0 \\
935.544,94.5\end{array}$ & 8 & Direct \\
\hline \multirow[t]{3}{*}{${ }^{55} \mathrm{Co}$} & ${ }^{58} \mathrm{Ni}$ & ${ }^{58} \mathrm{Ni}(\mathrm{p}, \alpha){ }^{55} \mathrm{Co}$ & 1.358 & ${ }^{55} \mathrm{Co}$ & $17.53 \mathrm{~h}$ & $\mathrm{EC}+\beta^{+} 100\left(\beta^{+} 76\right)$ & 10 & Direct \\
\hline & ${ }^{54} \mathrm{Fe}$ & ${ }^{54} \mathrm{Fe}(\mathrm{d}, \mathrm{n}){ }^{55} \mathrm{Co}$ & 0 & & & $\gamma: 931.1,75$ & 13 & Direct \\
\hline & ${ }^{56} \mathrm{Fe}$ & ${ }^{56} \mathrm{Fe}(\mathrm{p}, 2 \mathrm{n}){ }^{55} \mathrm{Co}$ & 15.71 & & & $1316.6,7.1$ & 8 & Direct \\
\hline \multirow[t]{3}{*}{${ }^{61} \mathrm{Cu}$} & ${ }^{61} \mathrm{Ni}$ & ${ }^{61} \mathrm{Ni}(\mathrm{p}, \mathrm{n}){ }^{61} \mathrm{Cu}$ & 3.07 & ${ }^{61} \mathrm{Cu}$ & $3.339 \mathrm{~h}$ & $\mathrm{EC}+\beta^{+} 100\left(\beta^{+} 61\right)$ & 12 & Direct \\
\hline & ${ }^{60} \mathrm{Ni}$ & ${ }^{60} \mathrm{Ni}(\mathrm{d}, \mathrm{n}){ }^{61} \mathrm{Cu}$ & 0 & & & $\gamma: 282.956,12.2$ & 16 & Direct \\
\hline & ${ }^{64} \mathrm{Zn}$ & ${ }^{64} \mathrm{Zn}(\mathrm{p}, \alpha){ }^{61} \mathrm{Cu}$ & 0 & & & $\begin{array}{l}656.008,10.8 \\
1185.234,3.7\end{array}$ & 12 & Direct \\
\hline \multirow[t]{4}{*}{${ }^{62} \mathrm{Cu}$} & ${ }^{\text {nat }} \mathrm{Cu}$ & ${ }^{63} \mathrm{Cu}(\mathrm{p}, 2 \mathrm{n}){ }^{62} \mathrm{Zn}^{\mathrm{b}}$ & 13.48 & ${ }^{62} \mathrm{Zn}$ & $9.193 \mathrm{~h}$ & $\mathrm{EC}+\beta^{+} 100\left(\beta^{+} 8.2\right)$ & 16 & Generator \\
\hline & ${ }^{\text {nat }} \mathrm{Cu}$ & ${ }^{63} \mathrm{Cu}(\mathrm{d}, 3 \mathrm{n}){ }^{62} \mathrm{Zn}^{\mathrm{b}}$ & 15.986 & & & $\gamma: 548.35,15.3$ & 12 & Generator \\
\hline & ${ }^{\mathrm{nat}} \mathrm{Ni}$ & ${ }^{n a t} \mathrm{Ni}(\alpha, \mathrm{xn})^{62} \mathrm{Zn}$ & 18.5 & & & $596.56,26$ & 10 & Generator \\
\hline & ${ }^{62} \mathrm{Ni}$ & ${ }^{62} \mathrm{Ni}(\mathrm{p}, \mathrm{n}){ }^{62} \mathrm{Cu}$ & 4.818 & ${ }^{62} \mathrm{Cu}$ & $9.67 \mathrm{~min}$ & $\mathrm{EC}+\beta^{+} 100\left(\beta^{+} 97.83\right)$ & 12 & Direct \\
\hline
\end{tabular}


Table 2 (continued)

\begin{tabular}{|c|c|c|c|c|c|c|c|c|}
\hline $\begin{array}{l}\text { PET } \\
\text { radionu- } \\
\text { clide }\end{array}$ & Target & Reaction & $\begin{array}{l}\text { Reaction } \\
\text { threshold } \\
(\mathrm{MeV})\end{array}$ & $\begin{array}{l}\text { Reaction } \\
\text { product }\end{array}$ & Half-life $^{\mathrm{a}}$ & $\begin{array}{l}\text { Decay }(\%)^{\mathrm{a}} \\
E_{\gamma}(\mathrm{keV}), P_{\gamma}(\%)\end{array}$ & $\begin{array}{l}\text { Order of Padé } \\
\text { polynomial L }\end{array}$ & Comment \\
\hline & ${ }^{62} \mathrm{Ni}$ & ${ }^{62} \mathrm{Ni}(\mathrm{d}, 2 \mathrm{n}){ }^{62} \mathrm{Cu}$ & 7.192 & & & $\begin{array}{l}\gamma: 875.66,0.147 \\
1172.97,0.342\end{array}$ & 5 & Direct \\
\hline \multirow[t]{2}{*}{${ }^{66} \mathrm{Ga}$} & ${ }^{66} \mathrm{Zn}$ & ${ }^{66} \mathrm{Zn}(\mathrm{p}, \mathrm{n}){ }^{66} \mathrm{Ga}$ & 6.049 & ${ }^{66} \mathrm{Ga}$ & $9.49 \mathrm{~h}$ & $\mathrm{EC}+\beta^{+} 100\left(\beta^{+} 57\right)$ & 13 & Direct \\
\hline & ${ }^{63} \mathrm{Cu}$ & ${ }^{63} \mathrm{Cu}(\alpha, n){ }^{66} \mathrm{Ga}$ & 7.980 & & & $\begin{array}{l}\gamma: 833.5324,5.9 \\
1039.220,37.0\end{array}$ & 13 & Direct \\
\hline \multirow[t]{4}{*}{${ }^{68} \mathrm{Ga}$} & ${ }^{68} \mathrm{Zn}$ & ${ }^{68} \mathrm{Zn}(\mathrm{p}, \mathrm{n}){ }^{68} \mathrm{Ga}$ & 3.758 & ${ }^{68} \mathrm{Ga}$ & $67.71 \mathrm{~min}$ & $\mathrm{EC}+\beta^{+} 100\left(\beta^{+} 88.91\right)$ & 20 & Direct \\
\hline & ${ }^{65} \mathrm{Cu}$ & ${ }^{65} \mathrm{Cu}(\alpha, \mathrm{n}){ }^{68} \mathrm{Ga}$ & 6.183 & & & $\gamma: 1077.34,3.22$ & 10 & Direct \\
\hline & ${ }^{\text {nat }} \mathrm{Ga}$ & ${ }^{\text {nat }} \mathrm{Ga}(\mathrm{p}, \mathrm{xn}){ }^{68} \mathrm{Ge}$ & 10.5 & ${ }^{68} \mathrm{Ge}$ & $270.95 \mathrm{~d}$ & EC 100 & 11 & Generator \\
\hline & ${ }^{69} \mathrm{Ga}$ & ${ }^{69} \mathrm{Ga}(\mathrm{p}, 2 \mathrm{n}){ }^{68} \mathrm{Ge}$ & 11.366 & & & From decay of ${ }^{68} \mathrm{Ga}$ & 8 & Generator \\
\hline \multirow[t]{4}{*}{${ }^{72} \mathrm{As}$} & ${ }^{75} \mathrm{As}$ & ${ }^{75} \mathrm{As}(\mathrm{p}, 4 \mathrm{n}){ }^{72} \mathrm{Se}$ & 30.57 & ${ }^{72} \mathrm{Se}$ & $8.40 \mathrm{~d}$ & EC 100 & 8 & Generator \\
\hline & ${ }^{\text {nat }} \mathrm{Br}$ & ${ }^{\text {nat }} \mathrm{Br}(\mathrm{p}, \mathrm{x}){ }^{72} \mathrm{Se}$ & 50 & & & From decay of ${ }^{72} \mathrm{As}$ & 10 & Generator \\
\hline & ${ }^{\text {nat }} \mathrm{Ge}$ & ${ }^{\text {nat }} \mathrm{Ge}(\mathrm{p}, \mathrm{xn})^{72} \mathrm{As}$ & 5 & ${ }^{72} \mathrm{As}$ & $26.0 \mathrm{~h}$ & $\mathrm{EC}+\beta^{+} 100\left(\beta^{+} 87.8\right)$ & 18 & Direct \\
\hline & ${ }^{\text {nat }} \mathrm{Ge}$ & ${ }^{\text {nat }} \mathrm{Ge}(\mathrm{d}, \mathrm{xn})^{72} \mathrm{As}$ & 8 & & & $\begin{array}{l}\gamma: 629.92,8.07 \\
833.99,81\end{array}$ & 10 & Direct \\
\hline \multirow[t]{2}{*}{${ }^{73} \mathrm{Se}$} & ${ }^{75} \mathrm{As}$ & ${ }^{75} \mathrm{As}(\mathrm{p}, 3 \mathrm{n}){ }^{73} \mathrm{Se}$ & 22.024 & ${ }^{73} \mathrm{Se}$ & $7.15 \mathrm{~h}$ & $\mathrm{EC}+\beta^{+} 100\left(\beta^{+} 65.4\right)$ & 9 & Direct \\
\hline & ${ }^{72} \mathrm{Ge}$ & ${ }^{72} \mathrm{Ge}(\alpha, 3 n){ }^{73} \mathrm{Se}$ & 27.60 & & & $\begin{array}{l}\gamma: 67.07,70 \\
361.2,97.0\end{array}$ & 8 & Direct \\
\hline \multirow[t]{3}{*}{${ }^{76} \mathrm{Br}$} & ${ }^{76} \mathrm{Se}$ & ${ }^{76} \mathrm{Se}(\mathrm{p}, \mathrm{n}){ }^{76} \mathrm{Br}$ & 5.821 & ${ }^{76} \mathrm{Br}$ & $16.2 \mathrm{~h}$ & $\mathrm{EC}+\beta^{+} 100\left(\beta^{+} 55\right)$ & 8 & Direct \\
\hline & ${ }^{77} \mathrm{Se}$ & ${ }^{77} \mathrm{Se}(\mathrm{p}, 2 \mathrm{n}){ }^{76} \mathrm{Br}$ & 13.336 & & & $\gamma: 559.09,74$ & 9 & Direct \\
\hline & ${ }^{75} \mathrm{As}$ & ${ }^{75} \mathrm{As}(\alpha, 3 \mathrm{n}){ }^{76} \mathrm{Br}$ & 25.84 & & & $\begin{array}{l}657.02,15.9 \\
1853.67,14.7\end{array}$ & 10 & Direct \\
\hline \multirow[t]{2}{*}{${ }^{82} \mathrm{Rb}$} & ${ }^{\mathrm{nat}} \mathrm{Rb}$ & ${ }^{\text {nat }} \mathrm{Rb}(\mathrm{p}, \mathrm{xn}){ }^{82} \mathrm{Sr}$ & 30 & ${ }^{82} \mathrm{Sr}$ & $25.35 \mathrm{~d}$ & EC 100 & 13 & Generator \\
\hline & ${ }^{85} \mathrm{Rb}$ & ${ }^{85} \mathrm{Rb}(\mathrm{p}, 4 \mathrm{n}){ }^{82} \mathrm{Sr}$ & 31.52 & & & $\begin{array}{l}\text { From decay of }{ }^{82} \mathrm{Rb}: \\
T_{1 / 2}=1.2575 \min \\
\mathrm{EC}+\beta^{+} 100\left(\beta^{+} 95.43\right) \\
\gamma: 776.52,15.08\end{array}$ & 9 & Generator \\
\hline \multirow[t]{2}{*}{${ }^{82 \mathrm{~m}} \mathrm{Rb}$} & ${ }^{82} \mathrm{Kr}$ & ${ }^{82} \mathrm{Kr}(\mathrm{p}, \mathrm{n}){ }^{82 \mathrm{~m}} \mathrm{Rb}$ & 5.250 & ${ }^{82 \mathrm{~m}} \mathrm{Rb}$ & $6.472 \mathrm{~h}$ & $\mathrm{EC}+\beta^{+} 100\left(\beta^{+} 21.2\right)$ & 9 & Direct \\
\hline & ${ }^{82} \mathrm{Kr}$ & ${ }^{82} \mathrm{Kr}(\mathrm{d}, 2 \mathrm{n}){ }^{82 \mathrm{~m}} \mathrm{Rb}$ & 7.593 & & & $\begin{array}{l}\gamma: 554.35,62.4 ; \\
619.11,37.98 ; \\
698.37,26.3 ; \\
776.52,84.39 ; \\
827.83,21.0 ; \\
1044.08,32.07 ; \\
1317.43,23.7 ; \\
1474.88,15.5\end{array}$ & 5 & Direct \\
\hline \multirow[t]{3}{*}{${ }^{86} \mathrm{Y}$} & ${ }^{86} \mathrm{Sr}$ & ${ }^{86} \mathrm{Sr}(\mathrm{p}, \mathrm{n}){ }^{86} \mathrm{Y}$ & 6.093 & ${ }^{86} \mathrm{Y}$ & $14.74 \mathrm{~h}$ & $\mathrm{EC}+\beta^{+} 100\left(\beta^{+} 31.9\right)$ & 9 & Direct \\
\hline & ${ }^{88} \mathrm{Sr}$ & ${ }^{88} \mathrm{Sr}(\mathrm{p}, 3 \mathrm{n}){ }^{86} \mathrm{Y}$ & 25.86 & & & $\gamma: 627.72,32.6$ & 8 & Direct \\
\hline & ${ }^{85} \mathrm{Rb}$ & ${ }^{85} \mathrm{Rb}(\alpha, 3 \mathrm{n}){ }^{86} \mathrm{Y}$ & 25.84 & & & $\begin{array}{l}1076.63,82.5 \\
1153.05,30.5\end{array}$ & 8 & Direct \\
\hline \multirow[t]{2}{*}{${ }^{89} \mathrm{Zr}$} & ${ }^{89} \mathrm{Y}$ & ${ }^{89} \mathrm{Y}(\mathrm{p}, \mathrm{n}){ }^{89} \mathrm{Zr}$ & 3.656 & ${ }^{89} \mathrm{Zr}$ & $78.41 \mathrm{~h}$ & $\mathrm{EC}+\beta^{+} 100\left(\beta^{+} 22.74\right)$ & 11 & Direct \\
\hline & ${ }^{89} \mathrm{Y}$ & ${ }^{89} \mathrm{Y}(\mathrm{d}, 2 \mathrm{n}){ }^{89} \mathrm{Zr}$ & 5.972 & & & $909.15,99.04$ & 9 & Direct \\
\hline \multirow[t]{2}{*}{${ }^{90} \mathrm{Nb}$} & ${ }^{93} \mathrm{Nb}$ & ${ }^{93} \mathrm{Nb}(\mathrm{p}, \mathrm{x}){ }^{90} \mathrm{Nb}$ & 29.08 & ${ }^{90} \mathrm{Nb}$ & $14.60 \mathrm{~h}$ & $\mathrm{EC}+\beta^{+} 100\left(\beta^{+} 51.2\right)$ & 9 & Direct \\
\hline & ${ }^{89} \mathrm{Y}$ & ${ }^{89} \mathrm{Y}(\alpha, 3 \mathrm{n}){ }^{90} \mathrm{Nb}$ & 28.04 & & & $\begin{array}{l}\gamma: 132.716,4.13 \\
141.178,66.8 \\
1129.224,92.7\end{array}$ & 16 & Direct \\
\hline \multirow[t]{2}{*}{${ }^{94 \mathrm{~m}} \mathrm{Tc}$} & ${ }^{92} \mathrm{Mo}$ & $\left.{ }^{92} \mathrm{Mo}(\alpha, \mathrm{x})\right)^{94 \mathrm{~m}} \mathrm{Tc}$ & 16.26 & ${ }^{94 \mathrm{~m}} \mathrm{Tc}$ & $52.0 \mathrm{~min}$ & $\mathrm{EC}+\beta^{+} 100\left(\beta^{+} 70.2\right)$ & 12 & Direct \\
\hline & ${ }^{94} \mathrm{Mo}$ & ${ }^{94} \mathrm{Mo}(\mathrm{p}, \mathrm{n}){ }^{94 \mathrm{~m}} \mathrm{Tc}$ & 5.092 & & & $\begin{array}{l}\gamma: 871.05,94.2 \\
1522.1,4.5 \\
1868.68,5.7\end{array}$ & 9 & Direct \\
\hline \multirow[t]{5}{*}{${ }_{110 \mathrm{~m}} \mathrm{In}$} & ${ }^{\text {nat In }}$ & ${ }^{\text {nat }} \operatorname{In}(p, x n){ }^{110} \mathrm{Sn}$ & 30 & ${ }^{110} \mathrm{Sn}$ & $4.154 \mathrm{~h}$ & EC 100 & 17 & Generator \\
\hline & ${ }^{108} \mathrm{Cd}$ & ${ }^{108} \mathrm{Cd}(\alpha, 2 \mathrm{n}){ }^{110} \mathrm{Sn}$ & 17.76 & & & $\gamma: 280.459,97.06$ & 10 & Generator \\
\hline & ${ }^{110} \mathrm{Cd}$ & ${ }^{110} \mathrm{Cd}(\mathrm{p}, \mathrm{n}){ }^{110 \mathrm{~m}} \mathrm{In}$ & 4.703 & ${ }^{110 \mathrm{~m}} \mathrm{In}$ & $69.1 \mathrm{~min}$ & $\mathrm{EC}+\beta^{+} 100\left(\beta^{+} 61.3\right)$ & 11 & Direct \\
\hline & ${ }^{110} \mathrm{Cd}$ & ${ }^{110} \mathrm{Cd}(\mathrm{d}, 2 \mathrm{n}){ }^{110 \mathrm{~m}} \mathrm{In}$ & 7.011 & & & $\gamma: 2129.40,2.15$ & 5 & Direct \\
\hline & ${ }^{107} \mathrm{Ag}$ & ${ }^{107} \mathrm{Ag}(\alpha, \mathrm{n}){ }^{110 \mathrm{~m}} \mathrm{In}$ & 7.867 & & & $\begin{array}{l}2211.33,1.74 \\
2317.41,1.285\end{array}$ & 9 & Direct \\
\hline \multirow[t]{2}{*}{${ }^{118} \mathrm{Sb}$} & ${ }^{115} \mathrm{Sn}$ & ${ }^{115} \mathrm{Sn}(\alpha, \mathrm{n}){ }^{118} \mathrm{Te}$ & 8.262 & ${ }^{118} \mathrm{Te}$ & $6.00 \mathrm{~d}$ & EC 100 & 7 & Generator \\
\hline & ${ }^{116} \mathrm{Sn}$ & ${ }^{116} \mathrm{Sn}(\alpha, 2 n){ }^{118} \mathrm{Te}$ & 18.15 & & & From decay of ${ }^{118} \mathrm{Sb}$ : & 6 & Generator \\
\hline
\end{tabular}


Table 2 (continued)

\begin{tabular}{|c|c|c|c|c|c|c|c|c|}
\hline $\begin{array}{l}\text { PET } \\
\text { radionu- } \\
\text { clide }\end{array}$ & Target & Reaction & $\begin{array}{l}\text { Reaction } \\
\text { threshold } \\
(\mathrm{MeV})\end{array}$ & $\begin{array}{l}\text { Reaction } \\
\text { product }\end{array}$ & Half-life $^{a}$ & $\begin{array}{l}\text { Decay }(\%)^{\mathrm{a}} \\
E_{\gamma}(\mathrm{keV}), P_{\gamma}(\%)\end{array}$ & $\begin{array}{l}\text { Order of Padé } \\
\text { polynomial L }\end{array}$ & Comment \\
\hline & ${ }^{\text {nat }} \mathrm{Sb}$ & ${ }^{\text {nat }} \mathrm{Sb}(\mathrm{p}, \mathrm{x}){ }^{118} \mathrm{Te}$ & 30 & & & $T_{1 / 2}=3.6 \mathrm{~min}$ & 12 & Generator \\
\hline & ${ }^{\text {nat }} \mathrm{Sb}$ & ${ }^{\text {nat }} \mathrm{Sb}(\mathrm{d}, \mathrm{x}){ }^{118} \mathrm{Te}$ & 34 & & & $\mathrm{EC}+\beta^{+} 100\left(\beta^{+} 73.5\right)$ & 4 & Generator \\
\hline & & & & & & $\gamma: 1229.33,2.5$ & & \\
\hline \multirow[t]{2}{*}{${ }^{120} \mathrm{I}$} & ${ }^{120} \mathrm{Te}$ & ${ }^{120} \mathrm{Te}(\mathrm{p}, \mathrm{n}){ }^{120} \mathrm{I}$ & 6.451 & ${ }^{120} \mathrm{I}$ & $81.6 \mathrm{~min}$ & $\mathrm{EC}+\beta^{+} 100\left(\beta^{+} 68.2\right)$ & 18 & Direct \\
\hline & ${ }^{122} \mathrm{Te}$ & ${ }^{122} \mathrm{Te}(\mathrm{p}, 3 \mathrm{n}){ }^{120} \mathrm{I}$ & 23.68 & & & $\begin{array}{l}\gamma: 601.1,5.51 \\
1523.0,10.9\end{array}$ & 7 & Direct \\
\hline \multirow[t]{3}{*}{${ }^{122} \mathrm{I}$} & ${ }^{124} \mathrm{Xe}$ & ${ }^{124} \mathrm{Xe}(\mathrm{p}, \mathrm{x}){ }^{122} \mathrm{Xe}$ & 18.60 & ${ }^{122} \mathrm{Xe}$ & $20.1 \mathrm{~h}$ & EC 100 & 5 & Generator \\
\hline & ${ }^{127} \mathrm{I}$ & ${ }^{127} \mathrm{I}(\mathrm{p}, 6 \mathrm{n}){ }^{122} \mathrm{Xe}$ & 45.12 & & & $\gamma: 350.065,7.80 ;$ and & 7 & Generator \\
\hline & ${ }^{127} \mathrm{I}$ & ${ }^{127} \mathrm{I}(\mathrm{d}, 7 \mathrm{n})^{122} \mathrm{Xe}$ & 47.74 & & & $\begin{array}{l}\text { from decay of }{ }^{122} \mathrm{I} \text { : } \\
T_{1 / 2}=3.63 \mathrm{~min} \\
\mathrm{EC}+\beta^{+} 100\left(\beta^{+} 78\right) \\
\gamma: 564.119,18\end{array}$ & 12 & Generator \\
\hline${ }^{128} \mathrm{Cs}$ & ${ }^{133} \mathrm{Cs}$ & ${ }^{133} \mathrm{Cs}(\mathrm{p}, 6 \mathrm{n}){ }^{128} \mathrm{Ba}$ & 44.16 & ${ }^{128} \mathrm{Ba}$ & $2.43 \mathrm{~d}$ & $\begin{array}{l}\text { EC } 100 \\
\gamma: 273.44,14.5 ; \text { and } \\
\text { from decay of }{ }^{128} \mathrm{Cs} \text { : } \\
T_{1 / 2}=3.66 \mathrm{~min} \\
\mathrm{EC}+\beta^{+} 100\left(\beta^{+} 68.8\right) \\
\gamma: 442.901,26.8\end{array}$ & 18 & Generator \\
\hline \multirow[t]{3}{*}{${ }^{140} \mathrm{Pr}$} & ${ }^{141} \mathrm{Pr}$ & ${ }^{141} \operatorname{Pr}(\mathrm{p}, 2 \mathrm{n}){ }^{140} \mathrm{Nd}$ & 10.68 & ${ }^{140} \mathrm{Nd}$ & $3.37 \mathrm{~d}$ & EC 100 & 10 & Generator \\
\hline & ${ }^{141} \mathrm{Pr}$ & ${ }^{141} \operatorname{Pr}(\mathrm{d}, 3 \mathrm{n}){ }^{140} \mathrm{Nd}$ & 13.02 & & & From decay of ${ }^{140} \mathrm{Pr}$ : & 9 & Generator \\
\hline & ${ }^{\text {nat }} \mathrm{Ce}$ & ${ }^{\text {nat }} \mathrm{Ce}\left({ }^{3} \mathrm{He}, \mathrm{xn}\right){ }^{140} \mathrm{Nd}$ & 16 & & & $\begin{array}{l}T_{1 / 2}=3.39 \mathrm{~min} \\
\mathrm{EC}+\beta^{+} 100\left(\beta^{+} 51\right) \\
\gamma: 306.9,0.147 \\
1596.1,0.49\end{array}$ & 9 & Generator \\
\hline
\end{tabular}

${ }^{a}$ Decay data as tabulated have been taken from NuDat (NuDat 2.6 or 2.7), a user-friendly form of ENSDF [4, 5]

${ }^{\mathrm{b}}$ Only contributing reaction in natural $\mathrm{Cu}$ targets at these energies 


\section{Production of ${ }^{449} \mathrm{Sc}\left(T_{1 / 2}=3.97 \mathrm{~h}\right)$ and long-lived ${ }^{44} \mathrm{Ti}$ parent $\left(T_{1 / 2}=59.1 \mathrm{y}\right)$}

Applications: ${ }^{44} \mathrm{Sc}$ (av. $E_{\beta+}=632.0 \mathrm{keV}, 94.27 \%$ intensity) has emerged as an attractive radiometal candidate for PET imaging by means of e.g., DOTA-functionalised biomolecules. ${ }^{44}$ Sc-labelled PET radiopharmaceuticals appear of interest for molecular imaging of medium-lasting physiological processes. Also forms a theranostic pair with therapeutic ${ }^{47} \mathrm{Sc}$.

${ }^{44} S c(3.97 h): \beta^{+}(94.27 \%)$, and $E_{\gamma}(\mathrm{keV})\left(P_{\gamma}(\%)\right): 1157.020$ (99.9).

${ }^{44} \mathrm{Ti}(59.1 \mathrm{y})$ : detected by means of radiation emitted by daughter ${ }^{44} \mathrm{Sc}$.

${ }^{44} \mathrm{Ca}(\mathrm{p}, \mathrm{n}){ }^{44} \mathrm{Sc},{ }^{44} \mathrm{Ca}(\mathrm{d}, 2 \mathrm{n}){ }^{44} \mathrm{Sc}$ and ${ }^{43} \mathrm{Ca}(\mathrm{d}, \mathrm{n}){ }^{44} \mathrm{Sc}$ direct reactions and ${ }^{45} \mathrm{Sc}(\mathrm{p}, 2 \mathrm{n}){ }^{44} \mathrm{Ti}-{ }^{44} \mathrm{Sc},{ }^{45} \mathrm{Sc}(\mathrm{d}, 3 \mathrm{n}){ }^{44} \mathrm{Ti}-{ }^{44} \mathrm{Sc}$ generator production routes were evaluated.

\section{${ }^{44} \mathrm{Ca}(p, n){ }^{449} \mathrm{Sc}$}

The six experimental datasets available in the literature are shown in Fig. 1 [33-38], together with the TENDL calculations. Three sets were rejected Cheng et al. [34] and Mitchell [35] (too high values near the threshold), and Krajewski et al. [37] (strange overall shape)), while the remaining four datasets were used in the statistical fitting procedure. Both the selected data and their experimental uncertainties are shown in Fig. 2 together with the Padé fit $(L=14, N=49$, $\mathrm{X}^{2}=1.63$ ) and estimated uncertainty in percentages, including $4 \%$ systematic uncertainty (right-hand scale).
Fig. 1 Six experimental datasets for the ${ }^{44} \mathrm{Ca}(\mathrm{p}, \mathrm{n})^{44} \mathrm{Sc}$ reaction available in the literature [33-38], and TENDL calculations

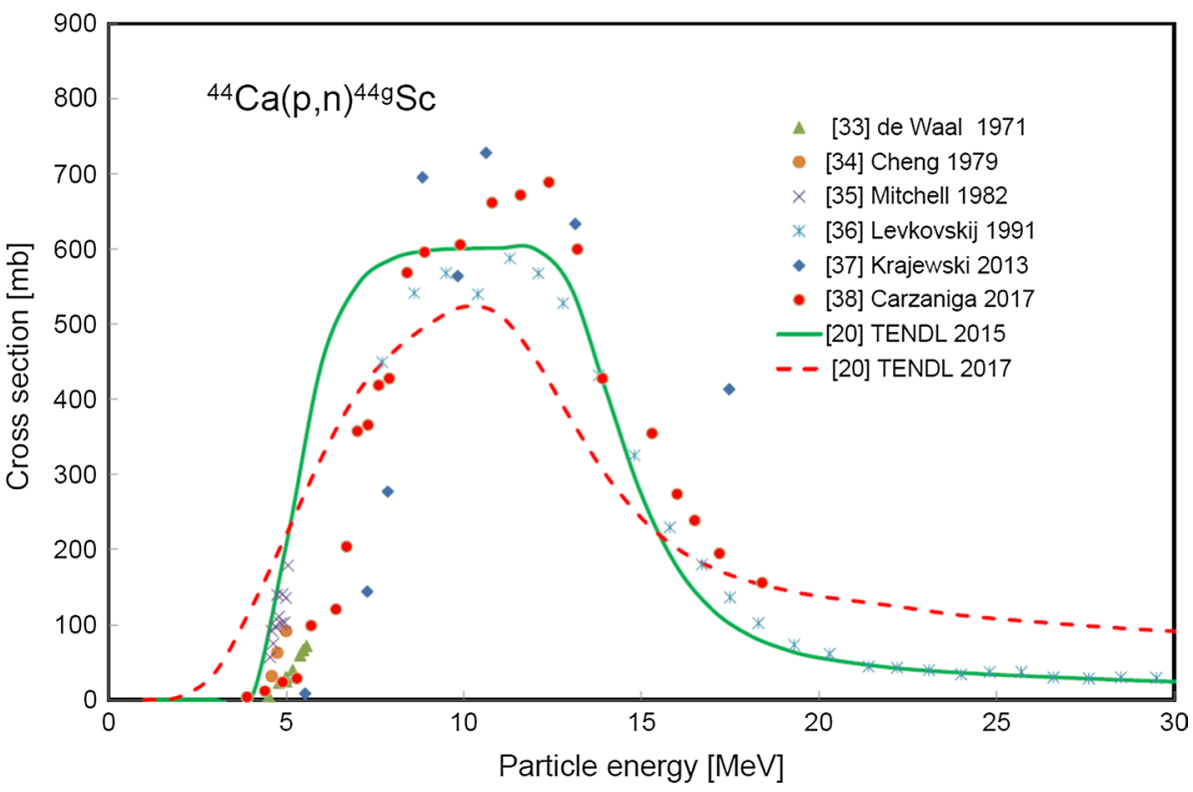


Fig. 2 Three selected experimental datasets for the ${ }^{44} \mathrm{Ca}(\mathrm{p}, \mathrm{n}){ }^{44} \mathrm{Sc}$ reaction $[33,36$, $38]$ with the Padé fit ( $L=19$, $N=56, \chi^{2}=1.86$, solid line), and estimated total uncertainties in percentages, including $4 \%$ systematic uncertainty (dashed line, right-hand scale)

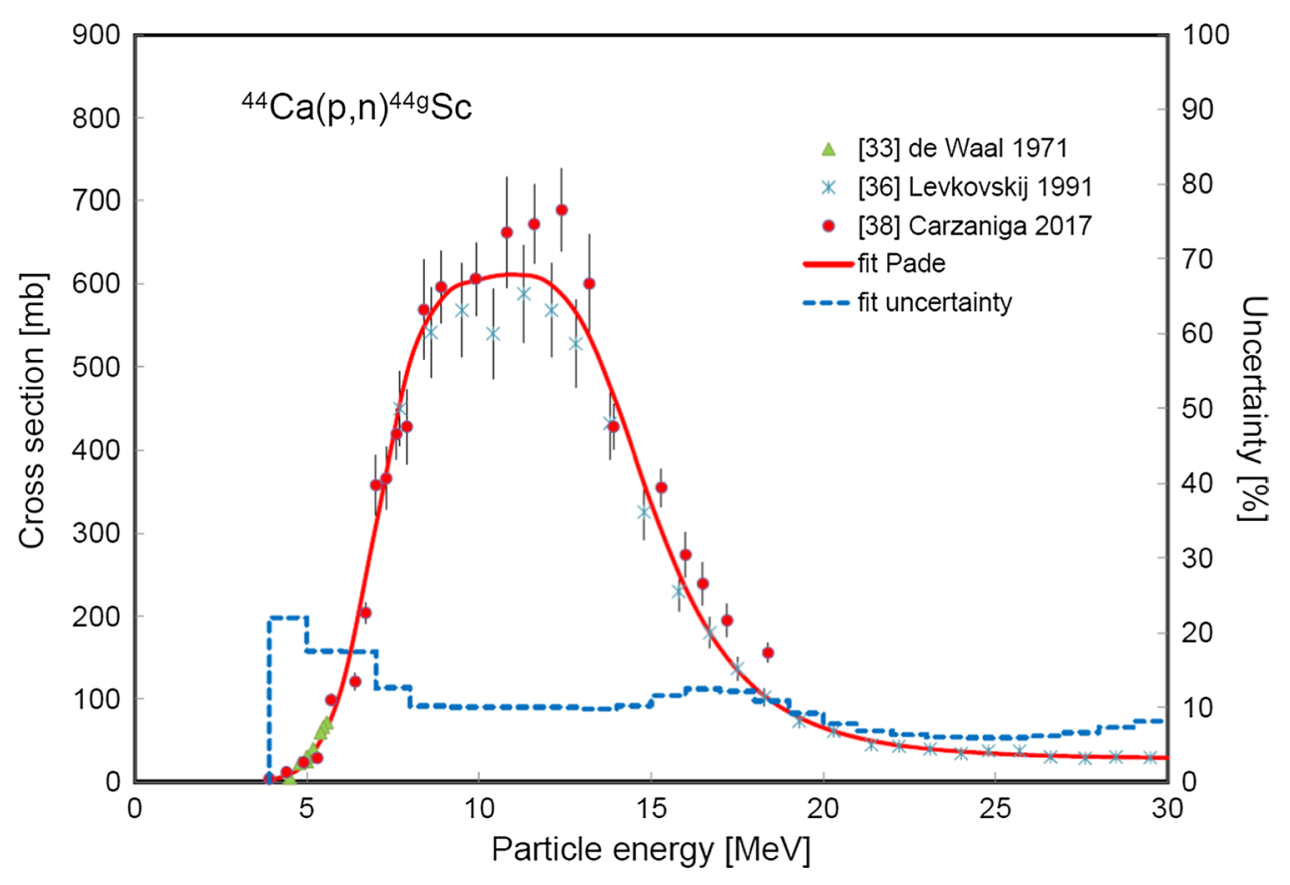


${ }^{44} \mathrm{Ca}(\mathrm{d}, 2 \mathrm{n}){ }^{44 \mathrm{~g}} \mathrm{Sc}$

Only one experimental dataset is available in the literature, and is shown in Fig. 3 [39] together with the TENDL calculations. These data and their experimental uncertainties are shown in Fig. 4 together with the Padé fit $(L=9, N=9$, $\chi^{2}=0.53$ ) and estimated uncertainty in percentages, including $4 \%$ systematic uncertainty (right-hand scale).
Fig. 3 One experimental dataset for the ${ }^{44} \mathrm{Ca}(\mathrm{d}, 2 \mathrm{n}){ }^{44 \mathrm{~g}} \mathrm{Sc}$ reaction available in the literature [39], and TENDL calculations

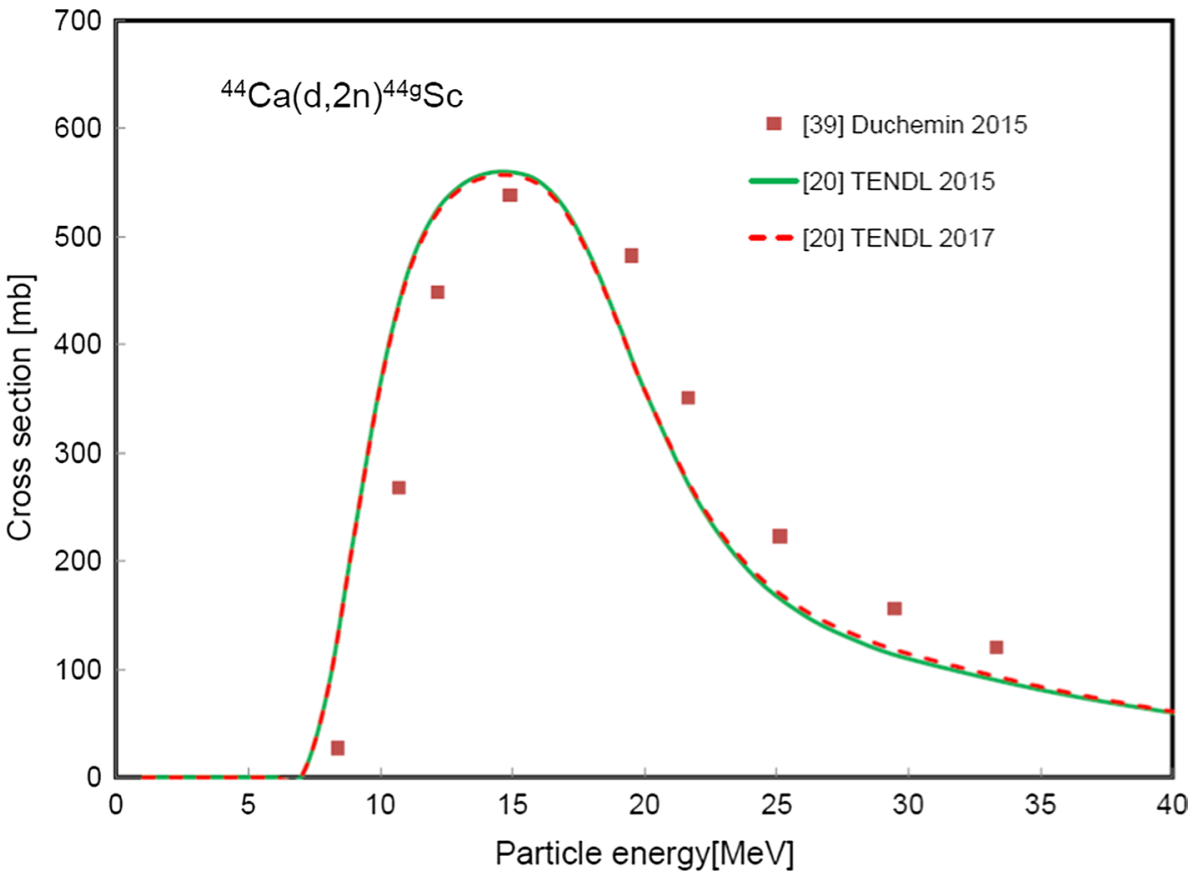

Fig. 4 Experimental dataset for the ${ }^{44} \mathrm{Ca}(\mathrm{d}, 2 \mathrm{n}){ }^{44 \mathrm{~g}} \mathrm{Sc}$ reaction [39] with the Padé fit $(L=9$, $N=9, \chi^{2}=0.53$, solid line) and estimated total uncertainties in percentages, including $4 \%$ systematic uncertainty (dashed line, right-hand scale)

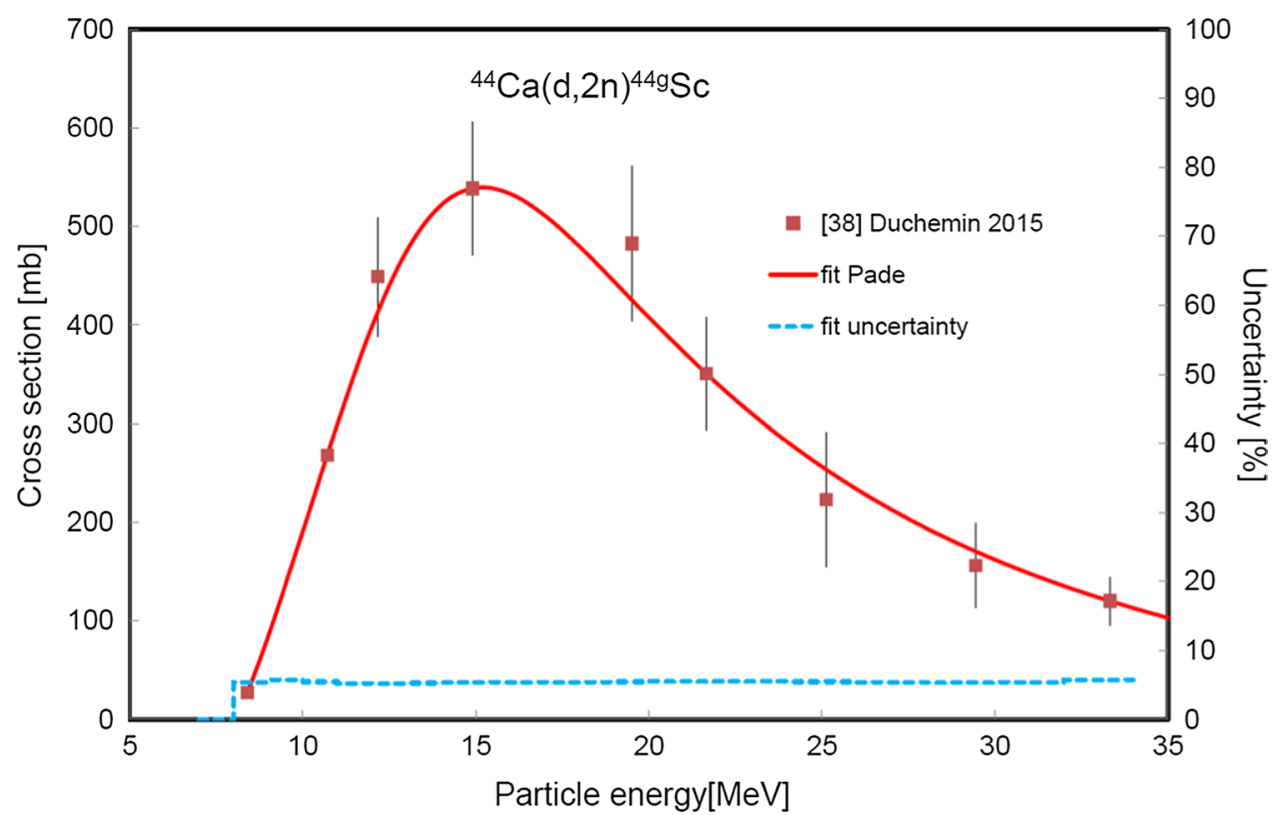




\section{${ }^{43} \mathrm{Ca}(\mathrm{d}, \mathrm{n}){ }^{449} \mathrm{Sc}$}

Only one experimental dataset is available in the literature, and is shown in Fig. 5 [40] together with the TENDL calculations. These data and their experimental uncertainties are shown in Fig. 6 together with the Padé fit $(L=5, N=16$, $\left.\chi^{2}=1.49\right)$ and estimated uncertainty in percentages, including $4 \%$ systematic uncertainty (right-hand scale).
Fig. 5 One experimental dataset for the ${ }^{43} \mathrm{Ca}(\mathrm{d}, \mathrm{n})^{44 \mathrm{~g}} \mathrm{Sc}$ reaction available in the literature [40], and TENDL calculations
Fig. 6 Experimental dataset for the ${ }^{43} \mathrm{Ca}(\mathrm{d}, \mathrm{n})^{44 \mathrm{~g}} \mathrm{Sc}$ reaction [40] with the Padé fit $(L=5$, $N=16, \chi^{2}=1.49$, solid line) and estimated total uncertainties in percentages, including $4 \%$ systematic uncertainty (dashed line, right-hand scale)

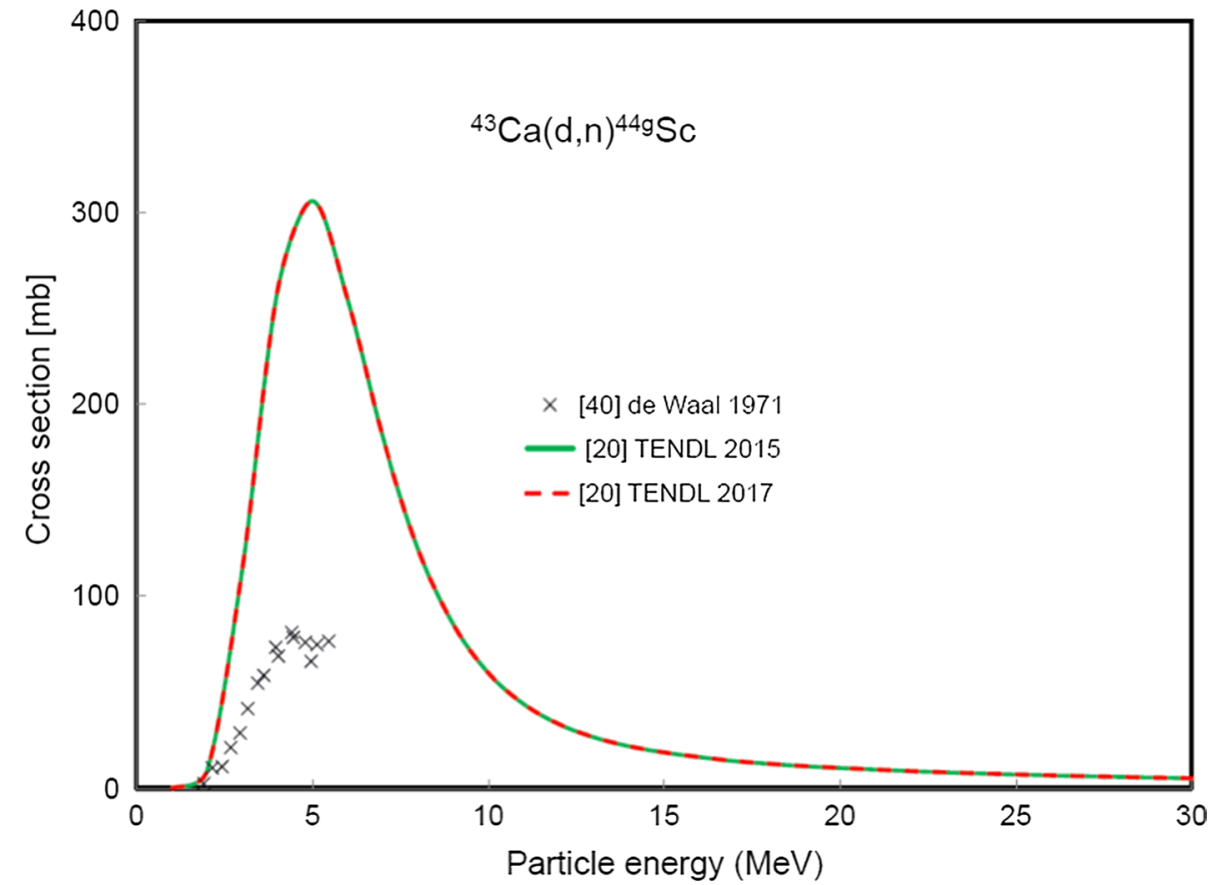


${ }^{45} \mathrm{Sc}(p, 2 \mathrm{n}){ }^{44} \mathrm{Ti}$

The five experimental datasets available in the literature are shown in Fig. 7 [36, 41-43] together with the TENDL calculations-Ref. [42] contains two sets labelled (a) and (b). Three datasets were rejected (both datasets of Ejnisman et al. [42], and McGee et al. [41] exhibit significant disagreement), while the remaining two datasets were used in the statistical fitting procedure. The selected data and their experimental uncertainties are shown in Fig. 8 together with the Padé fit $(L=7$, $N=26, \chi^{2}=1.58$ ) and estimated uncertainty in percentages, including $4 \%$ systematic uncertainty (right-hand scale).
Fig. 7 Five experimental datasets for the ${ }^{45} \mathrm{Sc}(\mathrm{p}, 2 \mathrm{n}){ }^{44} \mathrm{Ti}$ reaction available in the literature [36, 41-43], and TENDL calculations
Fig. 8 Two selected experimental datasets for the ${ }^{45} \mathrm{Sc}(\mathrm{p}, 2 \mathrm{n}){ }^{44} \mathrm{Ti}$ reaction $[36$, 43] with the Padé fit $(L=7$, $N=26, \chi^{2}=1.58$, solid line) and estimated total uncertainties in percentages, including $4 \%$ systematic uncertainty (dashed line, right-hand scale)
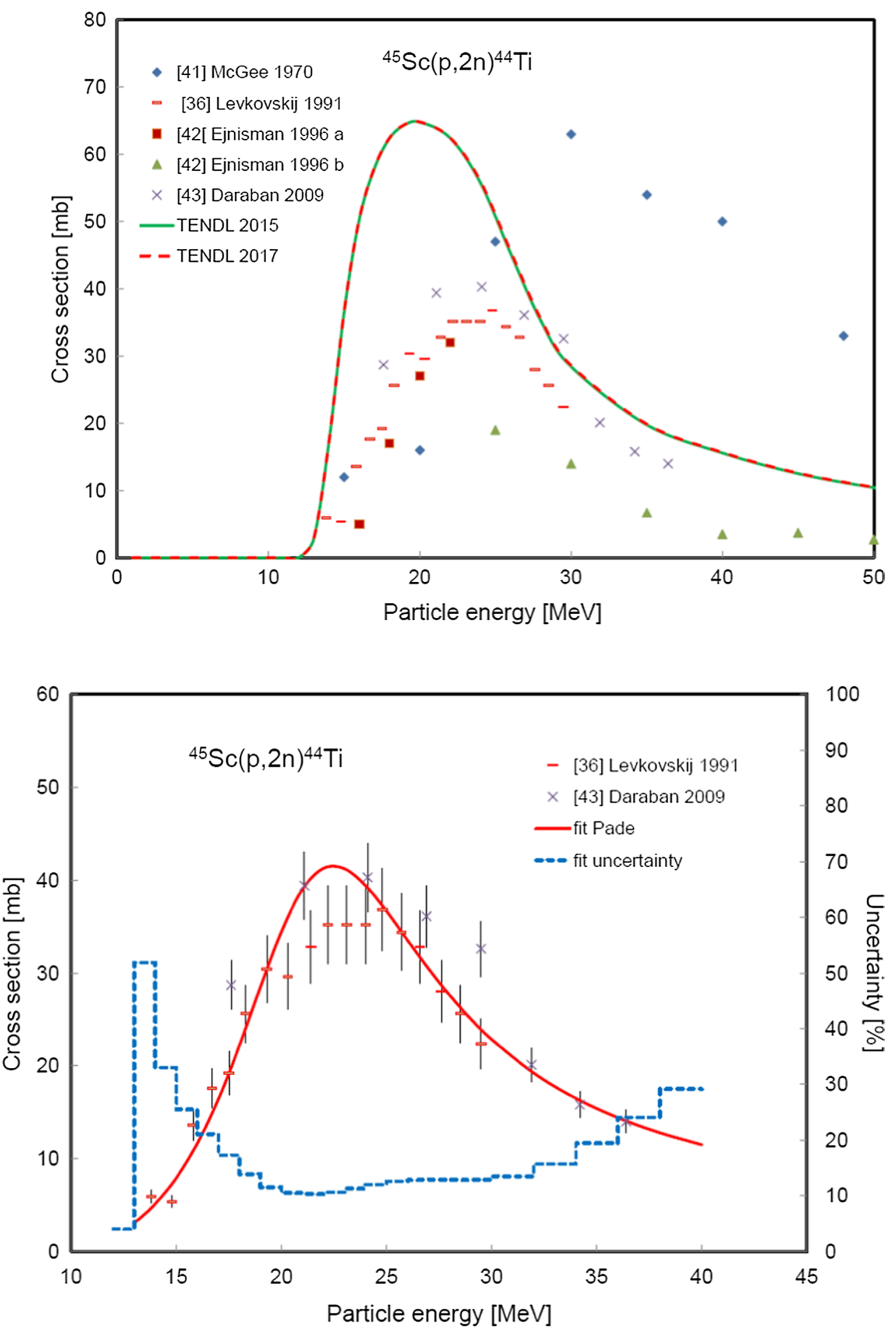


\section{${ }^{45} \mathrm{Sc}(\mathrm{d}, 3 \mathrm{n}){ }^{44} \mathrm{Ti}$}

Only one experimental dataset is available in the literature, and is shown in Fig. 9 [44] together with the TENDL calculations. These data and their experimental uncertainties are shown in Fig. 10 together with the Padé fit $(L=6, N=18$, $\left.\chi^{2}=0.406\right)$ and estimated uncertainty in percentages, including 4\% systematic uncertainty (right-hand scale).
Fig. 9 One experimental dataset for the ${ }^{45} \mathrm{Sc}(\mathrm{d}, 3 \mathrm{n}){ }^{44} \mathrm{Ti}$ reaction available in the literature [44], and TENDL calculations
Fig. 10 One experimental dataset for the ${ }^{45} \mathrm{Sc}(\mathrm{d}, 3 \mathrm{n})^{44} \mathrm{Ti}$ reaction [44] with the Padé fit $\left(L=6, N=18, \chi^{2}=0.406\right.$, solid line) and estimated total uncertainties in percentages, including $4 \%$ systematic uncertainty (dashed line, right-hand scale)
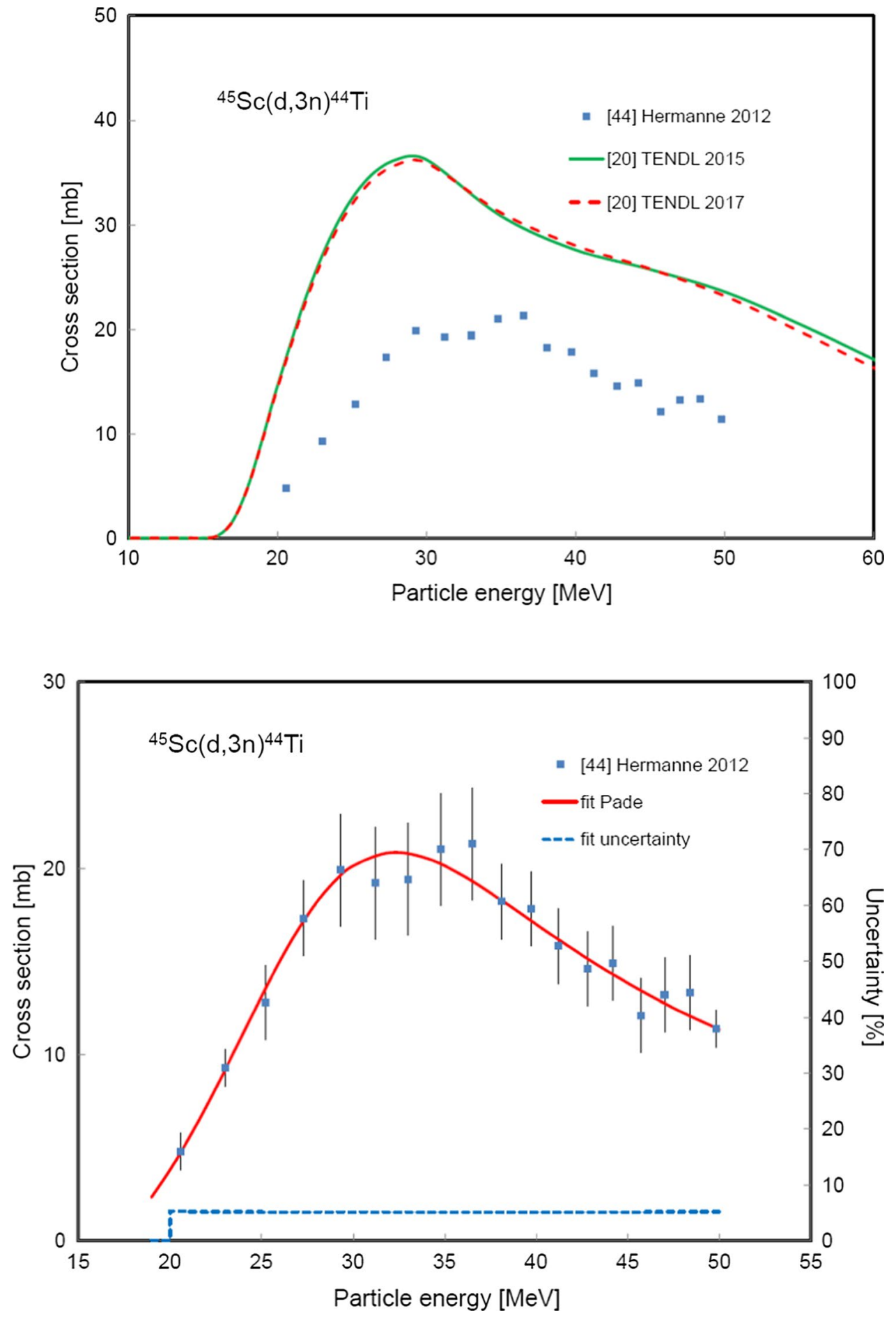
Thick target yields for production of ${ }^{449} S \mathrm{C}$, and long-lived

\section{${ }^{44} \mathrm{Ti}$ parent}

See Figs. 11 and 12.

Fig. 11 Thick target yields calculated from the recommended cross sections for the ${ }^{44} \mathrm{Ca}(\mathrm{p}, \mathrm{n}){ }^{44 \mathrm{~g}} \mathrm{Sc},{ }^{44} \mathrm{Ca}(\mathrm{d}, 2 \mathrm{n}){ }^{44 \mathrm{~g}} \mathrm{Sc}$ and ${ }^{43} \mathrm{Ca}(\mathrm{d}, \mathrm{n}){ }^{44 \mathrm{~g}} \mathrm{Sc}$ reactions
Fig. 12 Thick target yields calculated from the recommended cross sections for the ${ }^{45} \mathrm{Sc}(\mathrm{p}, 2 \mathrm{n})^{44} \mathrm{Ti}$ and ${ }^{45} \mathrm{Sc}(\mathrm{d}, 3 \mathrm{n}){ }^{44} \mathrm{Ti}$ reactions to produce long-lived parent for ${ }^{44}$ Sc generator
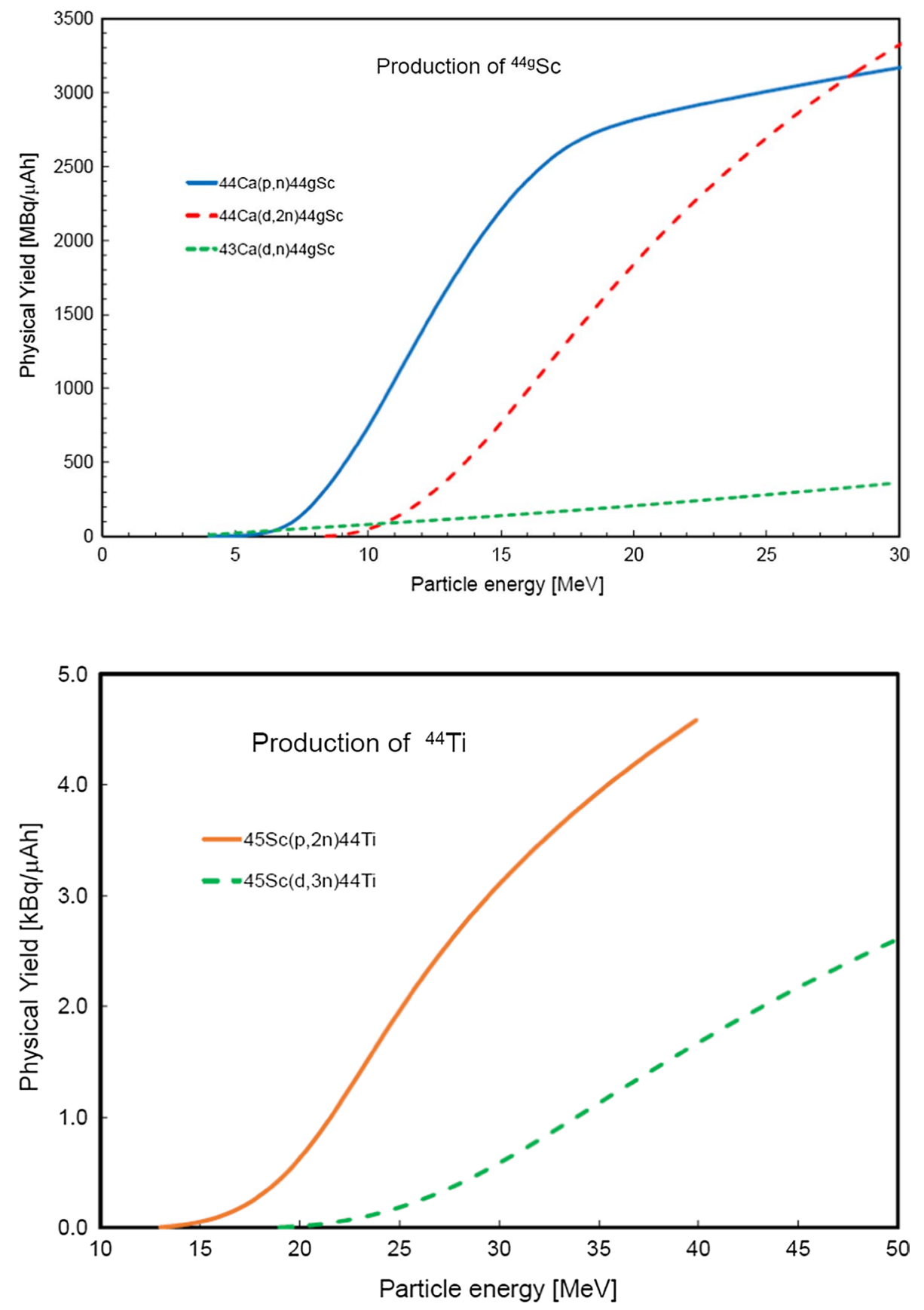


\section{Production of ${ }^{52 \mathrm{~m}} \mathrm{Mn}\left(T_{1 / 2}=21.1 \mathrm{~min}\right)$} and longer-lived ${ }^{52} \mathrm{Fe}$ parent $\left(T_{1 / 2}=8.275 \mathrm{~h}\right)$

Applications: ${ }^{52 \mathrm{~m}} \mathrm{Mn}$ has been suggested for myocardial and cerebral perfusion imaging, more recently for studies similar to Mn-enhanced neuronal MRI, and for diagnosis in other organ systems - bones, spinal cord and the digestive tract. ${ }^{52 m} \mathrm{Mn}(21.1 \mathrm{~min}): \beta^{+}(96.6 \%)$, and $E_{\gamma}(\mathrm{keV})\left(P_{\gamma}(\%)\right)$ : $1434.092(98.2)$.

${ }^{52} \mathrm{Fe}(8.275 \mathrm{~h})$ : detected by means of radiation emitted from daughter ${ }^{52 \mathrm{~m}} \mathrm{Mn}$.

Evaluations have been made of the ${ }^{52} \mathrm{Cr}(\mathrm{p}, \mathrm{n})^{52 \mathrm{~m}} \mathrm{Mn}$ and ${ }^{52} \mathrm{Cr}(\mathrm{d}, 2 \mathrm{n})^{52 \mathrm{~m}} \mathrm{Mn}$ direct production routes and ${ }^{\mathrm{nat}} \mathrm{Ni}(\mathrm{p}, \mathrm{x})^{52} \mathrm{Fe}$,
${ }^{55} \mathrm{Mn}(\mathrm{p}, 4 \mathrm{n}){ }^{52} \mathrm{Fe}$ and ${ }^{50} \mathrm{Cr}(\alpha, 2 \mathrm{n}){ }^{52} \mathrm{Fe}$ reactions for indirect production through decay of the longer-lived parent.

${ }^{\text {nat }} \mathrm{Ni}(\mathrm{p}, \mathrm{x})^{52} \mathrm{Fe}$

The four experimental datasets available in the literature are shown in Fig. 13 [45-48] together with he TENDL calculations. One set was rejected (Titarenko et al. [47], values too high), and the remaining three datasets were used in the statistical fitting procedure. These selected data and their experimental uncertainties are shown in Fig. 14 together with the Padé fit ( $\left.L=11, N=41, \chi^{2}=0.57\right)$ and estimated uncertainty in percentages, including $4 \%$ systematic uncertainty (right-hand scale).
Fig. 13 Four experimental datasets for the ${ }^{\text {nat }} \mathrm{Ni}(\mathrm{p}, \mathrm{x})^{52} \mathrm{Fe}$ reaction available in the literature [45-48], and TENDL calculations

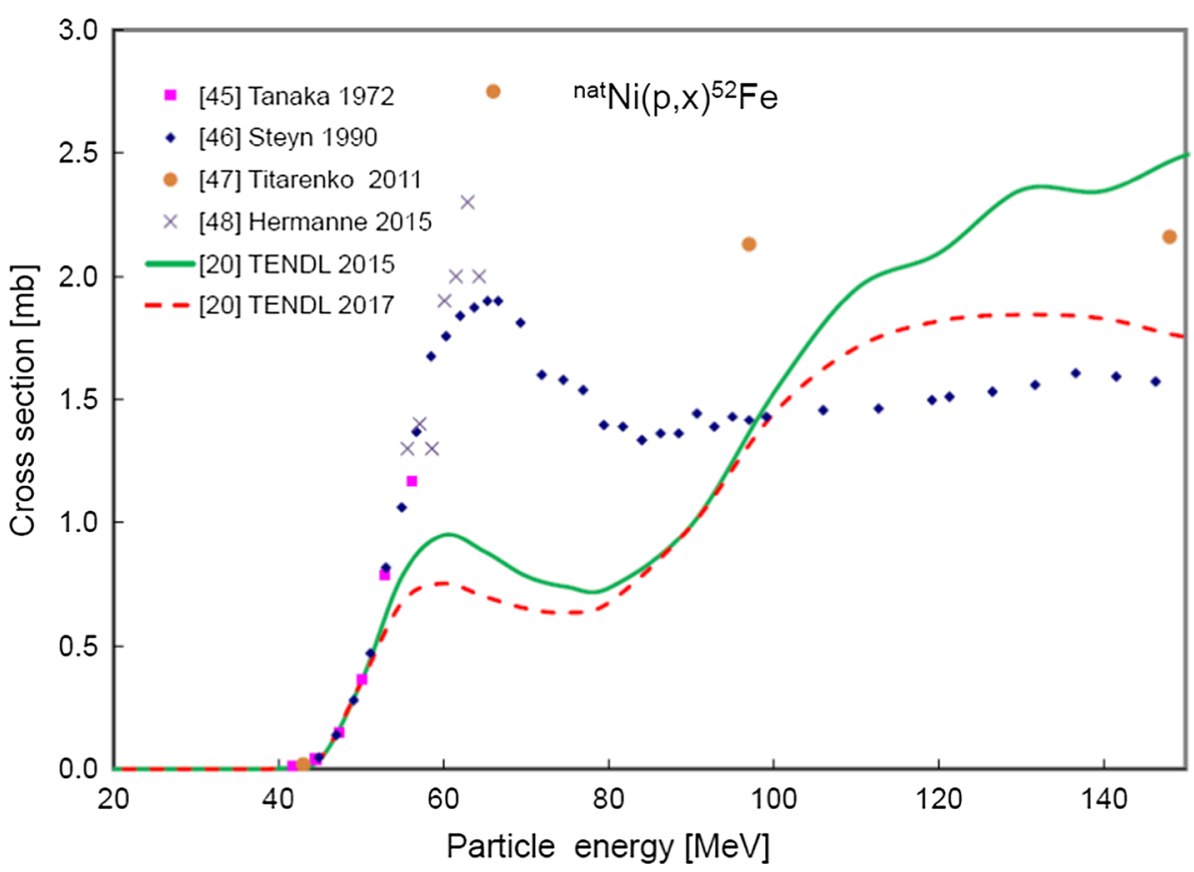


Fig. 14 Three selected experimental datasets for the ${ }^{\text {nat }} \mathrm{Ni}(\mathrm{p}, \mathrm{x})^{52} \mathrm{Fe}$ reaction $[45,46$, 48] with the Padé fit $(L=11$, $N=41, \chi^{2}=0.57$, solid line) and estimated total uncertainties in percentages, including $4 \%$ systematic uncertainty (dashed line, right-hand scale)

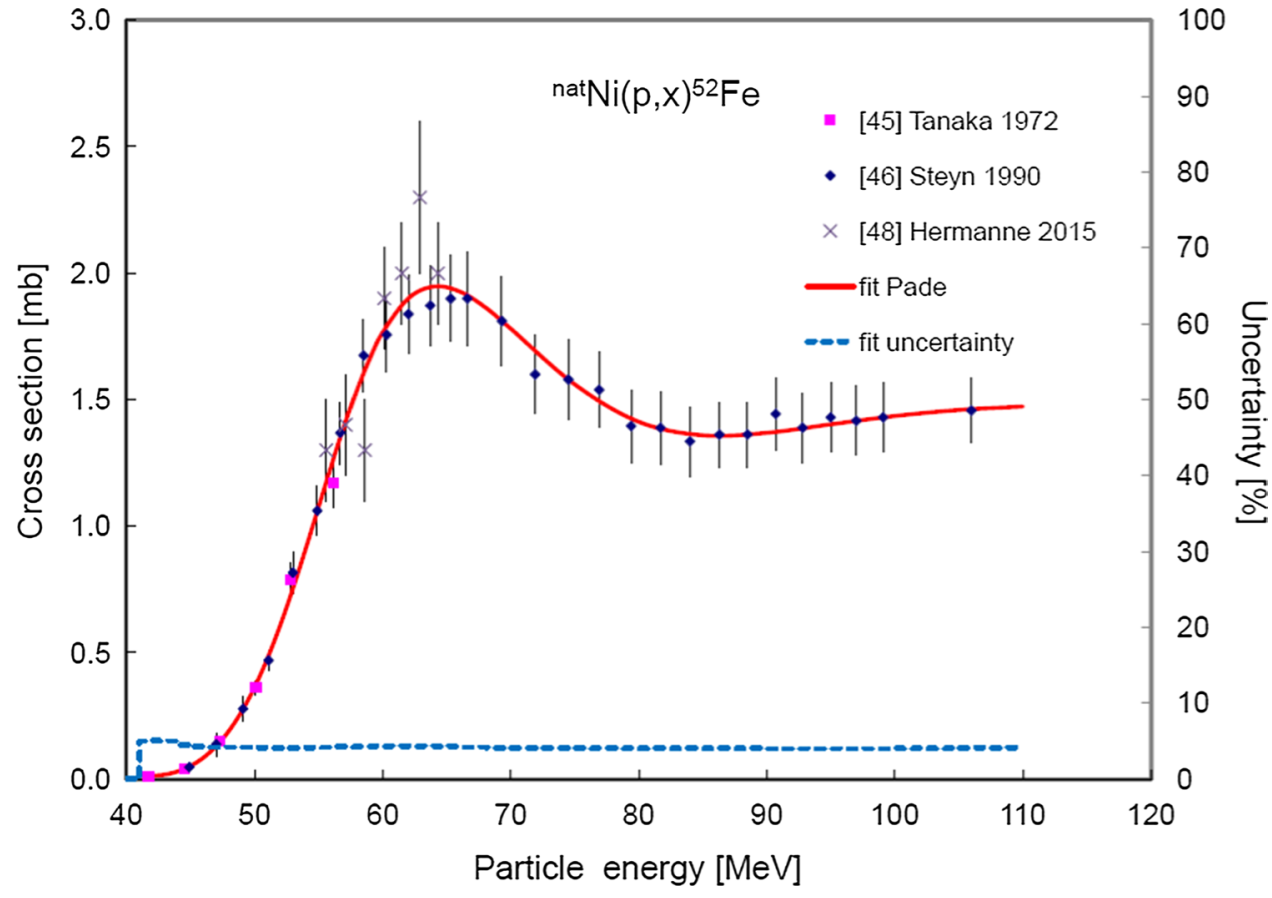




\section{${ }^{55} \mathrm{Mn}(\mathrm{p}, 4 \mathrm{n}){ }^{52} \mathrm{Fe}$}

The four experimental datasets available in the literature are shown in Fig. 15 [46, 49-51] together with the TENDL calculations. All sets were used for the statistical fitting procedure. These data and their experimental uncertainties are shown in Fig. 16 together with the Padé fit $(L=17$, $\left.N=157, \chi^{2}=1.01\right)$ and estimated uncertainty in percentages, including $4 \%$ systematic uncertainty (right-hand scale).
Fig. 15 Four experimental datasets for the ${ }^{55} \mathrm{Mn}(\mathrm{p}, 4 \mathrm{n})^{52} \mathrm{Fe}$ reaction available in the literature [46, 49-51], and TENDL calculations
Fig. 16 Four experimental datasets for the ${ }^{55} \mathrm{Mn}(\mathrm{p}, 4 \mathrm{n})^{52} \mathrm{Fe}$ reaction $[46,49-51]$ with the Padé fit $(L=17, N=157$, $\chi^{2}=1.01$, solid line) and estimated total uncertainties in percentages, including $4 \%$ systematic uncertainty (dashed line, right-hand scale)
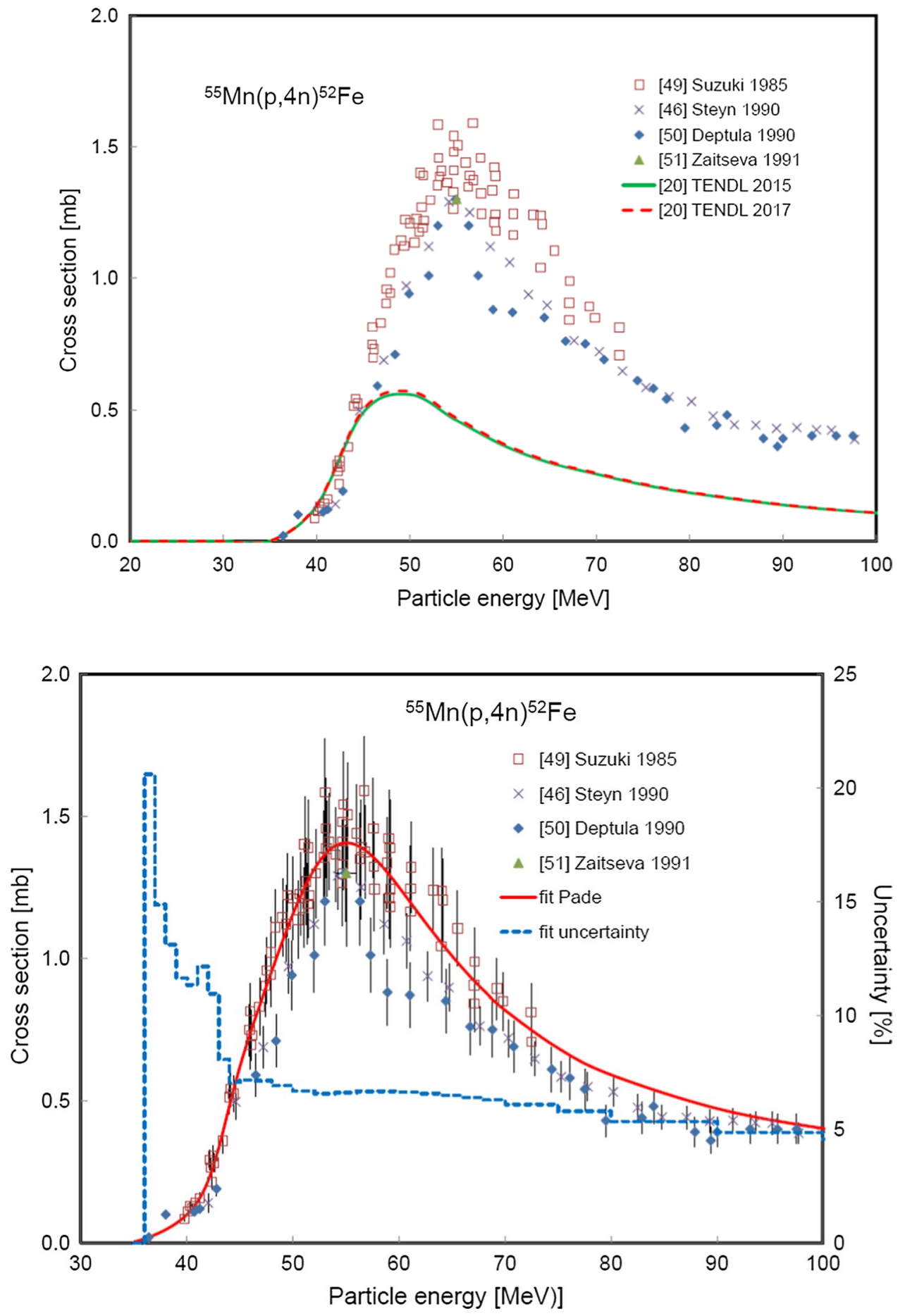
${ }^{50} \mathrm{Cr}(a, 2 \mathrm{n}){ }^{52} \mathrm{Fe}$

The four experimental datasets available in the literature are shown in Fig. 17 [36, 52-54] together with the TENDL calculations. Two datasets were rejected (Akiha et al. [52], energy shift; Chowdhury et al. [53], unusual shape,with one outlying data point at 27.3 MeV not represented in Fig. 17), while the remaining two datasets were used in the statistical fitting procedure. Both the selected data and their experimental uncertainties are shown in Fig. 18 together with the Padé fit ( $L=9, N=52, \chi^{2}=0.616$, solid line) and estimated uncertainty in percentages, including $4 \%$ systematic uncertainty (right-hand scale).
Fig. 17 Four experimental datasets for the ${ }^{50} \mathrm{Cr}(\alpha, 2 \mathrm{n}){ }^{52} \mathrm{Fe}$ reaction available in the literature [36, 52-54], and TENDL calculations
Fig. 18 Two selected experimental datasets for the ${ }^{50} \mathrm{Cr}(\alpha, 2 \mathrm{n}){ }^{52} \mathrm{Fe}$ reaction [36, 54] with the Padé fit $(L=9$, $N=52, \chi^{2}=0.616$, solid line) and estimated total uncertainties in percentages, including $4 \%$ systematic uncertainty (dashed line, right-hand scale)
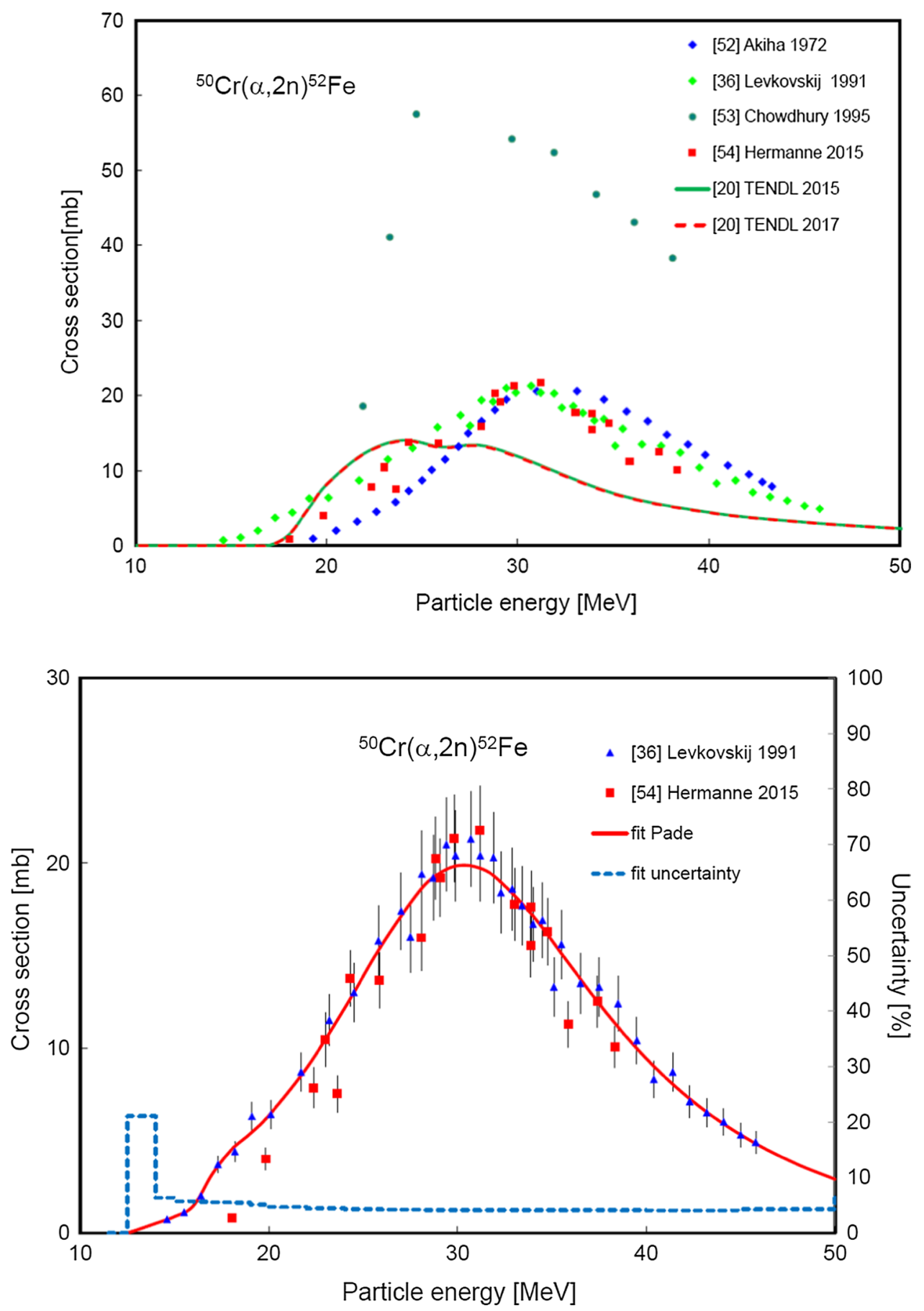


\section{${ }^{52} \mathrm{Cr}(\mathrm{p}, \mathrm{n})^{52 \mathrm{~m}} \mathrm{Mn}$}

The nine experimental datasets available in the literature are shown in Fig. 19 [36, 55-62] together with the TENDL calculations. Three datasets were rejected (Blosser and Handley [56], Wing and Huizenga [58], and West et al. [62], all values too high), while the remaining six datasets were used in the statistical fitting procedure. Both the selected data and their experimental uncertainties are shown in Fig. 20 together with the Padé fit $\left(L=14, N=68, \chi^{2}=1.15\right)$ and estimated uncertainty in percentages, including $4 \%$ systematic uncertainty (right-hand scale).
Fig. 19 Nine experimental datasets for the ${ }^{52} \mathrm{Cr}(\mathrm{p}, \mathrm{n}){ }^{52 \mathrm{~m}} \mathrm{Mn}$ reaction available in the literature [36, 55-62], and TENDL calculations
Fig. 20 Six selected experimental datasets for the ${ }^{52} \mathrm{Cr}(\mathrm{p}, \mathrm{n}){ }^{52 \mathrm{~m}} \mathrm{Mn}$ reaction [36, 55, 57, 59-61] with the Padé fit $\left(L=14, N=68, \chi^{2}=1.15\right.$, solid line) and estimated total uncertainties in percentages, including $4 \%$ systematic uncertainty (dashed line, right-hand scale)
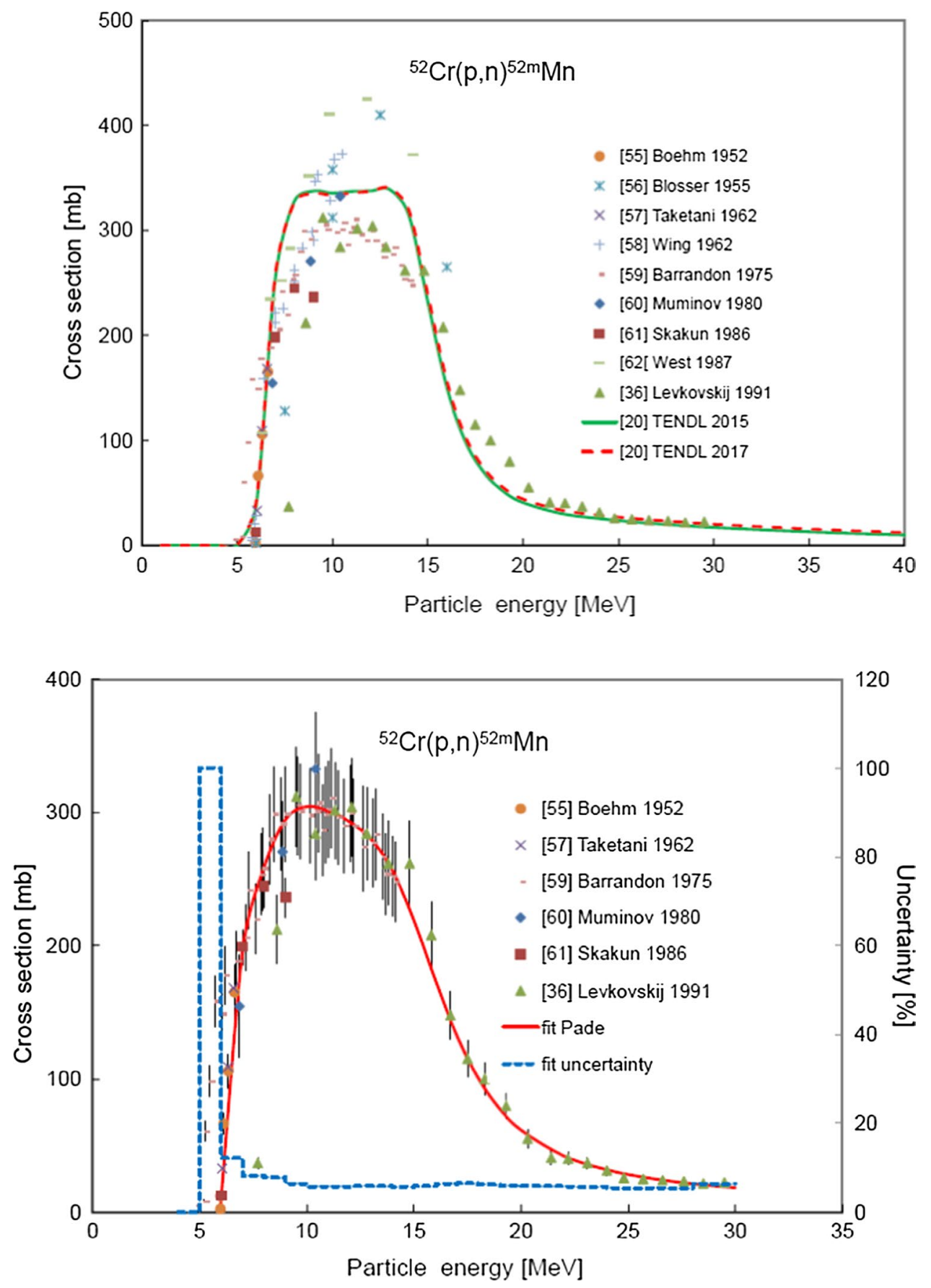


\section{${ }^{52} \mathrm{Cr}(\mathrm{d}, 2 \mathrm{n}){ }^{52 \mathrm{~m}} \mathrm{Mn}$}

The two experimental datasets available in the literature are shown in Fig. 21 [62, 63] together with the TENDL calculations. Both datasets were used for the statistical fitting procedure. These data and their experimental uncertainties are shown in Fig. 22 together with the Padé fit $(L=8, N=16$, $\chi^{2}=0.71$ ) and estimated uncertainty in percentages, including $4 \%$ systematic uncertainty (right-hand scale).
Fig. 21 Two experimental datasets for the ${ }^{52} \mathrm{Cr}(\mathrm{d}, 2 \mathrm{n}){ }^{52 \mathrm{~m}} \mathrm{Mn}$ reaction available in the literature [62, 63], and TENDL calculations

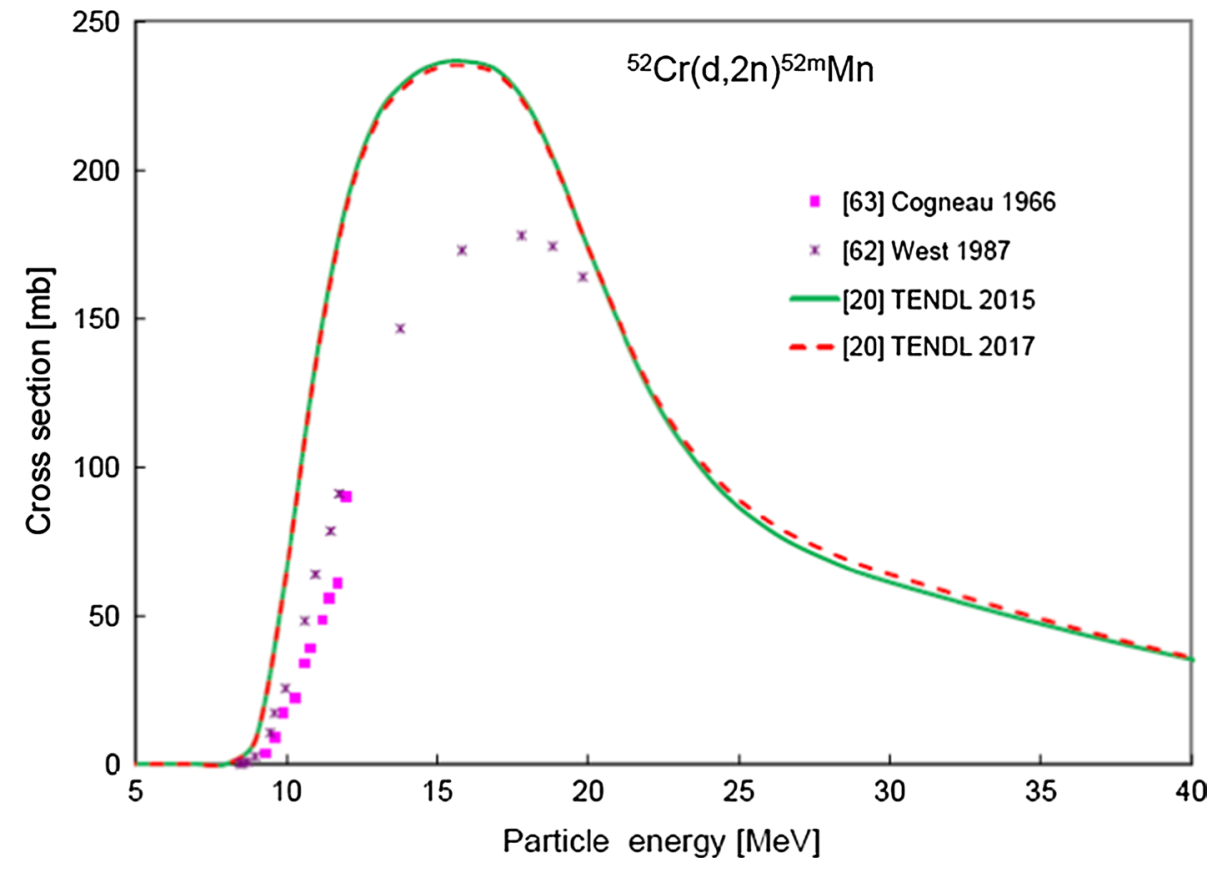

Fig. 22 Two experimental datasets for the ${ }^{52} \mathrm{Cr}(\mathrm{d}, 2 \mathrm{n}){ }^{52 \mathrm{~m}} \mathrm{Mn}$ reaction $[62,63]$ with the Padé fit $\left(L=8, N=16, \chi^{2}=0.71\right.$, solid line) and estimated total uncertainties in percentages, including $4 \%$ systematic uncertainty (dashed line, right-hand scale)

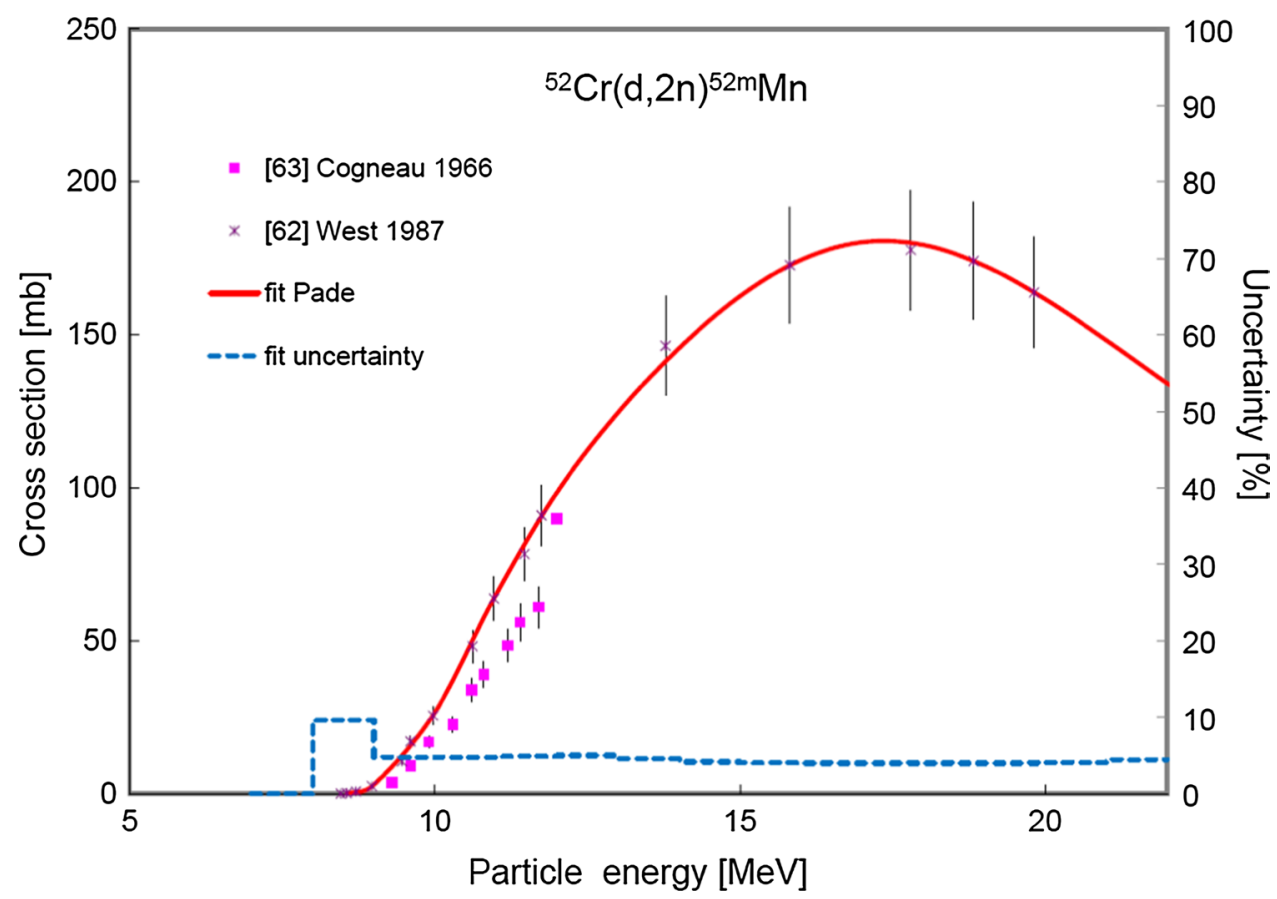


Thick target yields for production of ${ }^{52 \mathrm{~m}} \mathrm{Mn}$, and long-lived

${ }^{52} \mathrm{Fe}$ parent

See Figs. 23 and 24.

Fig. 23 Thick target yields calculated from the recommended cross sections for the ${ }^{52} \mathrm{Cr}(\mathrm{p}, \mathrm{n})^{52 \mathrm{~m}} \mathrm{Mn}$ and

${ }^{52} \mathrm{Cr}(\mathrm{d}, 2 \mathrm{n}){ }^{52 \mathrm{~m}} \mathrm{Mn}$ reactions

Fig. 24 Thick target yields calculated from the recommended cross sections for the ${ }^{\text {nat }} \mathrm{Ni}(\mathrm{p}, \mathrm{x})^{52} \mathrm{Fe},{ }^{55} \mathrm{Mn}(\mathrm{p}, 4 \mathrm{n})^{52} \mathrm{Fe}$ and ${ }^{50} \mathrm{Cr}(\alpha, 2 \mathrm{n})^{52} \mathrm{Fe}$ parent reactions
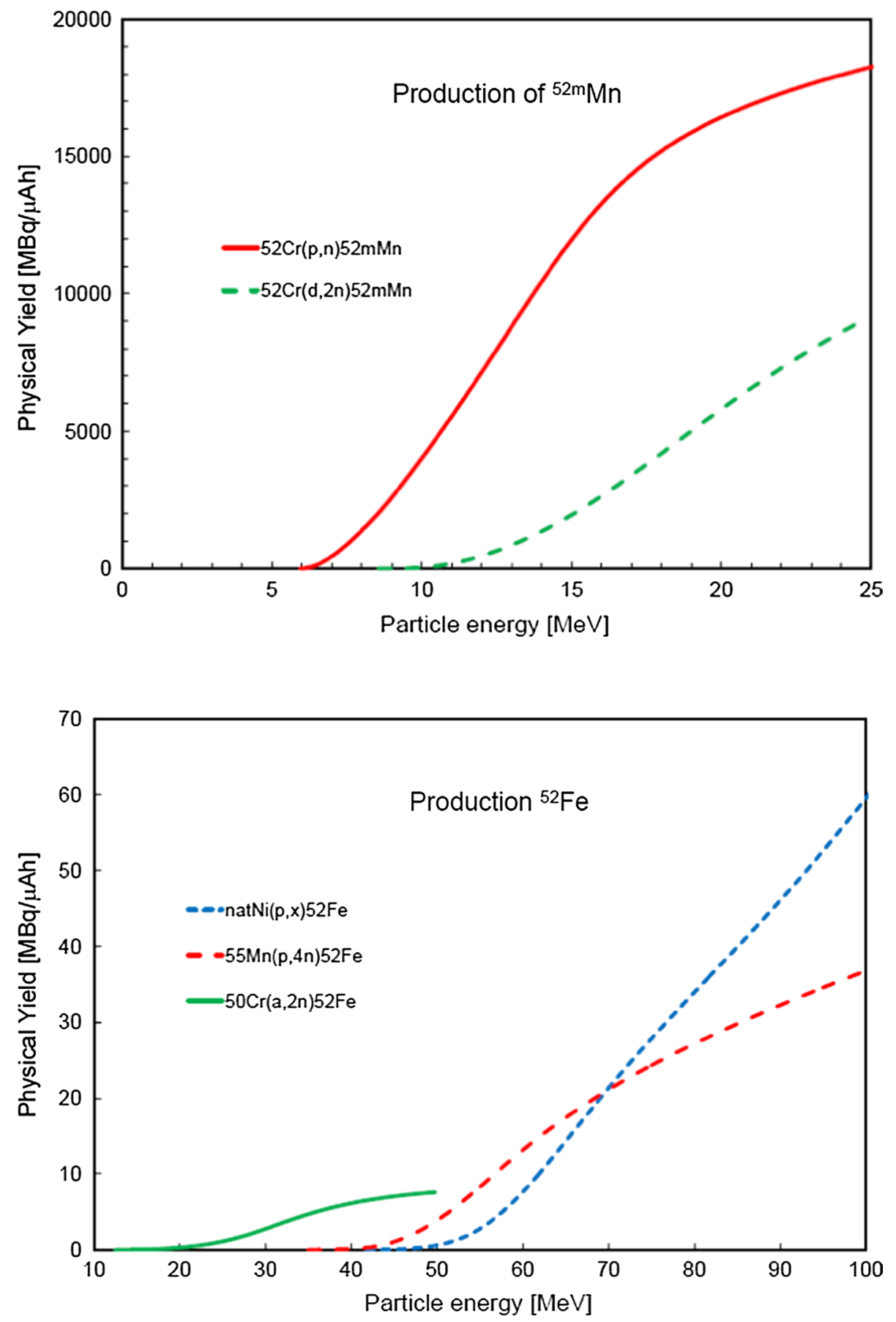


\section{Production of ${ }^{52 \mathrm{~g}} \mathrm{Mn}\left(T_{1 / 2}=5.591 \mathrm{~d}\right)$}

Applications: The longer-lived ${ }^{52} \mathrm{Mn}$ ground state has potential as a PET tracer for preclinical in vivo neuroimaging and other applications such as cell tracking, immuno-PET and functional $\beta$-cell mass quantification. Unfortunately, a half-life of $5.591 \mathrm{~d}$ coupled with an extremely high radiation burden that arises from the resulting gamma-ray emissions has limited ${ }^{52 \mathrm{~g}} \mathrm{Mn}$ clinical applications.

${ }^{52 g} \mathrm{Mn}(5.591 \mathrm{~d}): \beta^{+}(29.4 \%)$, and $E_{\gamma}(\mathrm{keV})\left(P_{\gamma}(\%)\right): 744.233$ (90.0), 935.544 (94.5), 1434.092 (100).

Evaluations have been made of the direct ${ }^{52} \mathrm{Cr}(\mathrm{p}, \mathrm{n}){ }^{52 \mathrm{~g}} \mathrm{Mn}(\mathrm{m}+)$ and ${ }^{52} \mathrm{Cr}(\mathrm{d}, 2 \mathrm{n}){ }^{52 \mathrm{~g}} \mathrm{Mn}(\mathrm{m}+)$ production routes, including the partial decay of the simultaneously produced short-lived ${ }^{52 \mathrm{~m}} \mathrm{Mn}$ metastable state (IT $=1.78 \%$, noted as $(\mathrm{m}+))$ which has already been assessed and discussed in section "Production of ${ }^{52 \mathrm{~m}} \mathrm{Mn}\left(T_{1 / 2}=21.1 \mathrm{~min}\right)$ and longerlived ${ }^{52} \mathrm{Fe}$ parent $\left(T_{1 / 2}=8.275 \mathrm{~h}\right)$ ".
${ }^{52} \mathrm{Cr}(\mathrm{p}, \mathrm{n}){ }^{52 \mathrm{~g}} \mathrm{Mn}(\mathrm{m}+)$

The thirteen experimental datasets available in the literature are shown in Fig. 25 [36, 55, 56, 58, 59, 61, 62, 64-69] together with the TENDL calculations. Six sets of data were rejected (Blosser and Handley [56], Tanaka and Furukawa [64], Lindner and James [65], Antropov et al. [66], Buchholz et al. [67], and Zherebchevsky et al. [69], all disagree significantly with the other datasets), while the remaining seven datasets were used in the statistical fitting procedure. These selected data and their experimental uncertainties are shown in Fig. 26 together with the Padé fit $\left(L=9, N=103, \chi^{2}=1.84\right)$ and estimated uncertainty in percentages, including $4 \%$ systematic uncertainty (righthand scale).
Fig. 25 Thirteen experimental datasets for the ${ }^{52} \mathrm{Cr}(\mathrm{p}, \mathrm{n}){ }^{52 \mathrm{~g}} \mathrm{Mn}(\mathrm{m}+)$ reaction available in the literature [36, $55,56,58,59,61,62,64-69]$, and TENDL calculations

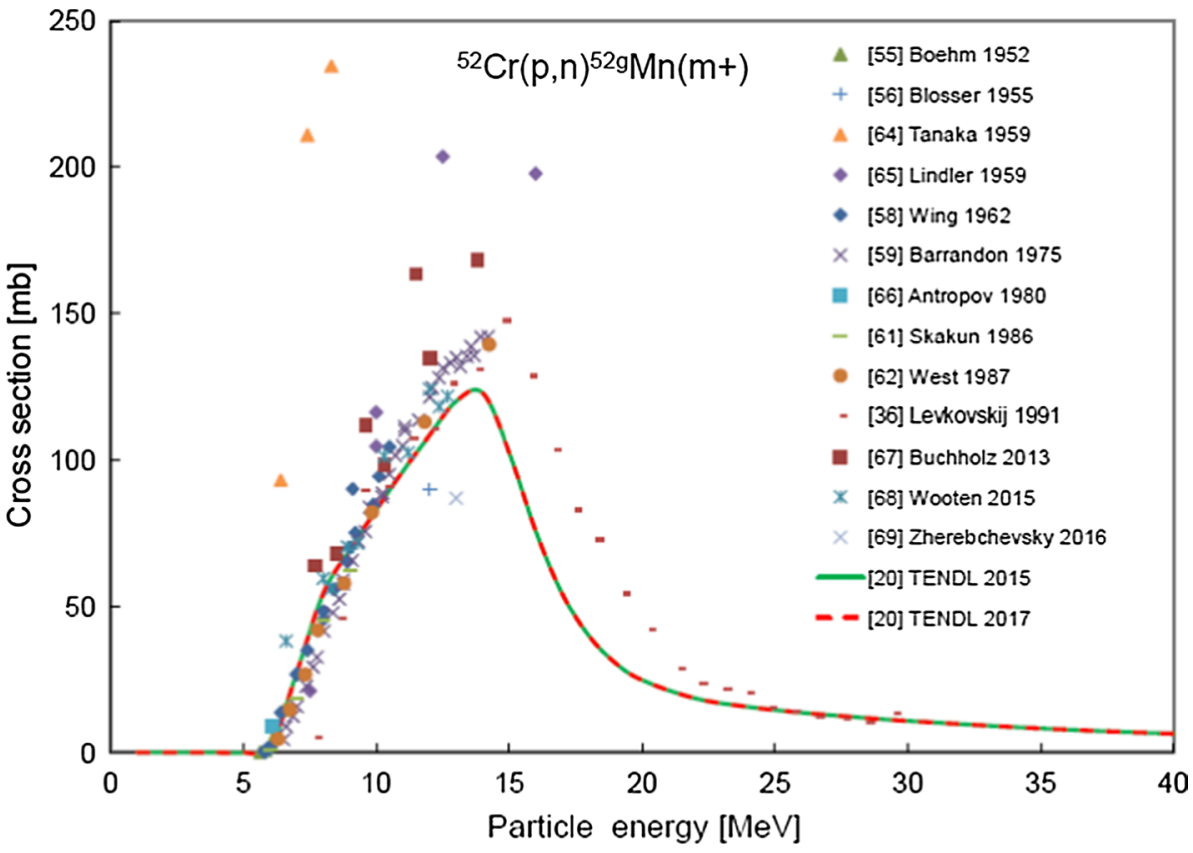


Fig. 26 Seven selected experimental datasets for the ${ }^{52} \mathrm{Cr}(\mathrm{p}, \mathrm{n}){ }^{52 \mathrm{~g}} \mathrm{Mn}(\mathrm{m}+)$ reaction $[36,55,58,59,61,62$, 68 with the Padé fit ( $L=9$, $N=103, \chi^{2}=1.84$, solid line) and estimated total uncertainties in percentages, including $4 \%$ systematic uncertainty (dashed line, right-hand scale)

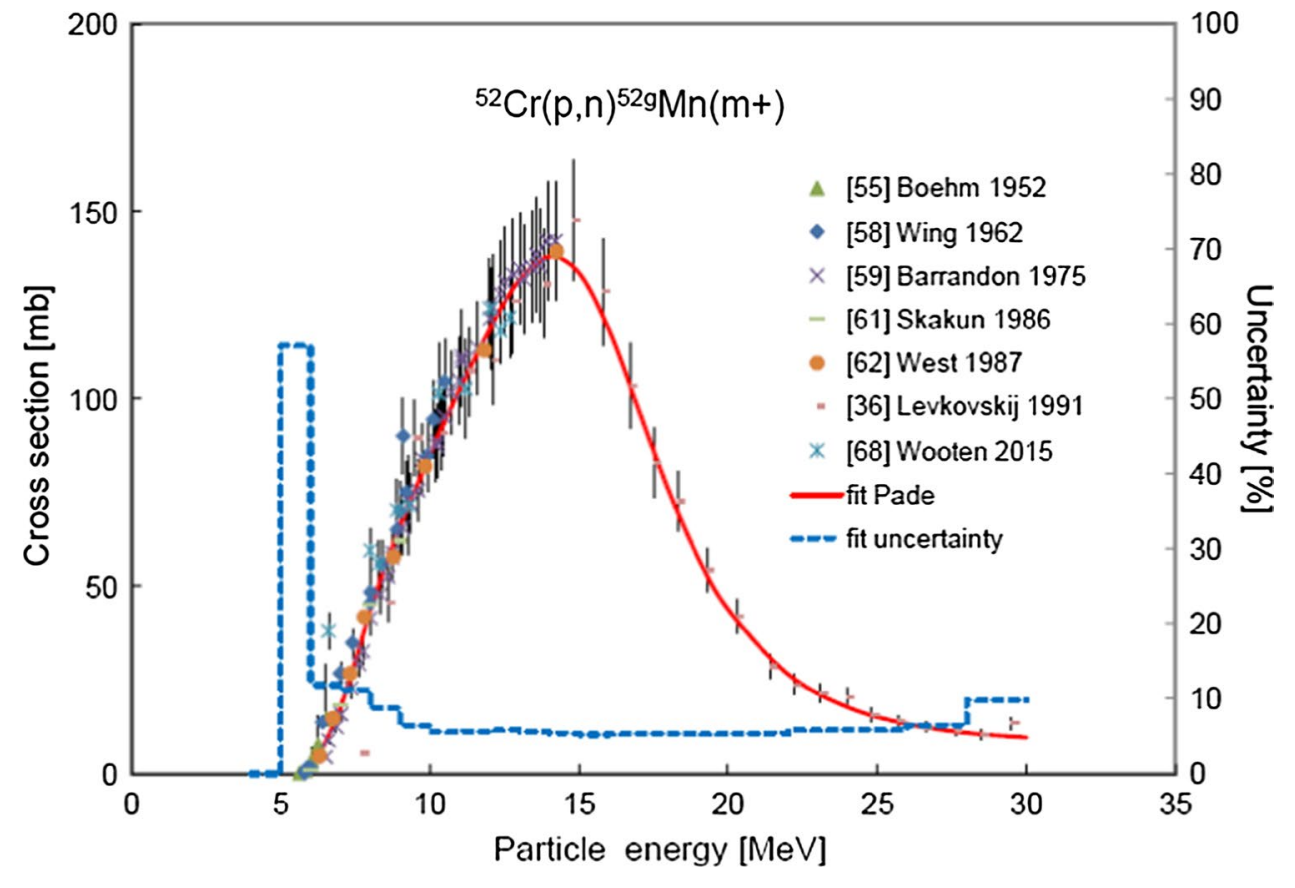




\section{${ }^{52} \mathrm{Cr}(\mathrm{d}, 2 \mathrm{n}){ }^{52 \mathrm{~g}} \mathrm{Mn}(\mathrm{m}+)$}

The six experimental datasets available in the literature are shown in Fig. 27 [62, 63, 70-73] together with the TENDL calculations. Two sets were rejected (Cheng Xiaowu et al. [71], values too low and no contribution from decay of metastable state marked as "g" in Fig. 27, and Nassiff and Münzel
[72], values too high), and the remaining four datasets were used in the statistical fitting procedure. These selected data and their experimental uncertainties are shown in Fig. 28 together with the Padé fit $\left(L=8, N=36, \chi^{2}=0.54\right)$ and estimated uncertainty in percentages, including $4 \%$ systematic uncertainty (right-hand scale).
Fig. 27 Six experimental datasets for the ${ }^{52} \mathrm{Cr}(\mathrm{d}, 2 \mathrm{n}){ }^{52 \mathrm{~g}} \mathrm{Mn}(\mathrm{m}+)$ reaction available in the literature $[62,63,70-73]$, and TENDL calculations
Fig. 28 Four selected experimental datasets for the ${ }^{52} \mathrm{Cr}(\mathrm{d}, 2 \mathrm{n}){ }^{52 \mathrm{~g}} \mathrm{Mn}(\mathrm{m}+)$ reaction $[62,63,70,73]$ with the Padé fit $\left(L=8, N=36, \chi^{2}=0.54\right.$, solid line) and estimated total uncertainties in percentages, including $4 \%$ systematic uncertainty (dashed line, right-hand scale)
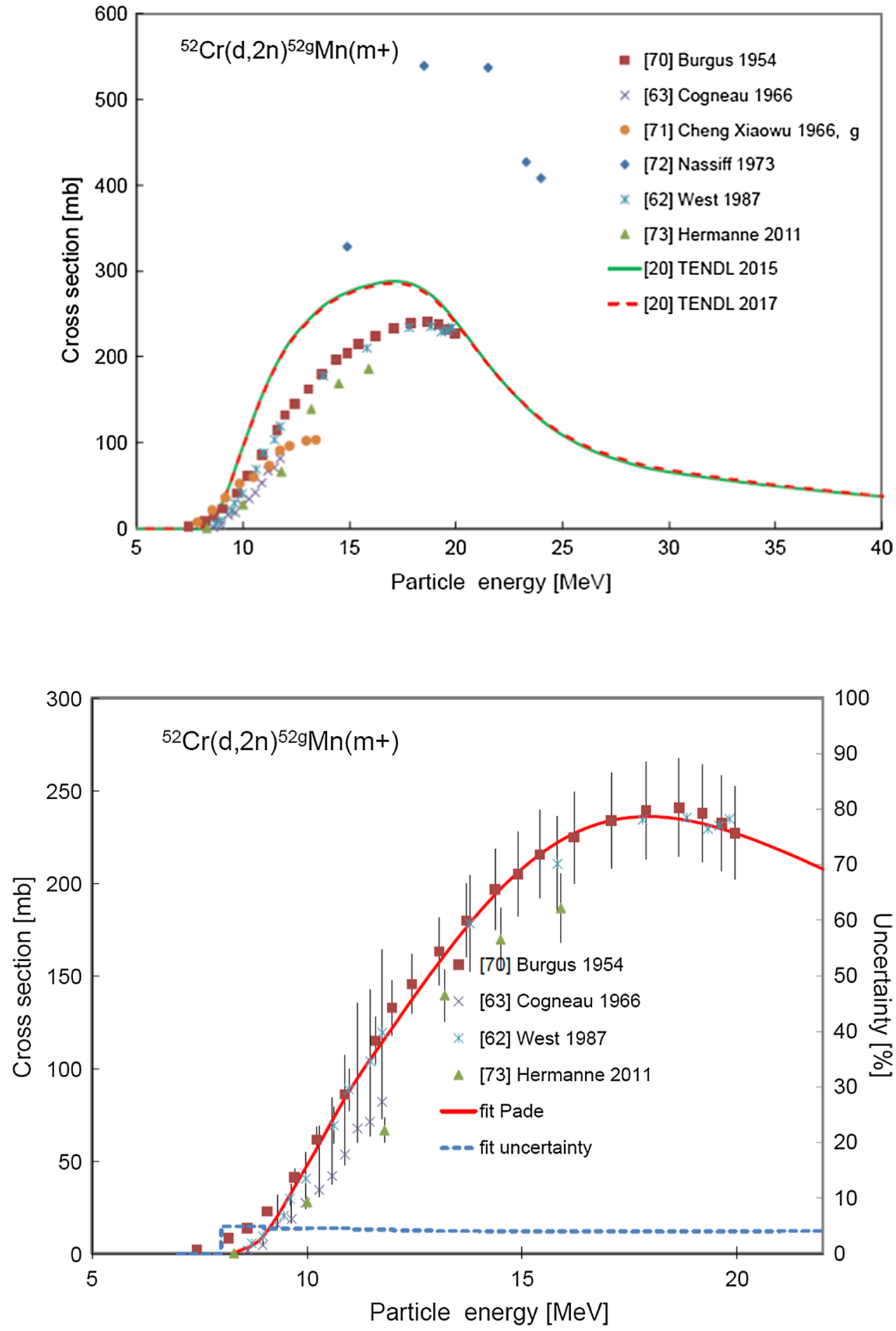


\section{Thick target yields for production of ${ }^{529} \mathrm{Mn}(\mathrm{m}+)$}

See Fig. 29.

Fig. 29 Thick target yields calculated from the recommended cross sections for the ${ }^{52} \mathrm{Cr}(\mathrm{p}, \mathrm{n})^{52 \mathrm{~g}} \mathrm{Mn}(\mathrm{m}+)$ and ${ }^{52} \mathrm{Cr}(\mathrm{d}, 2 \mathrm{n}){ }^{52 \mathrm{~g}} \mathrm{Mn}(\mathrm{m}+)$ reactions

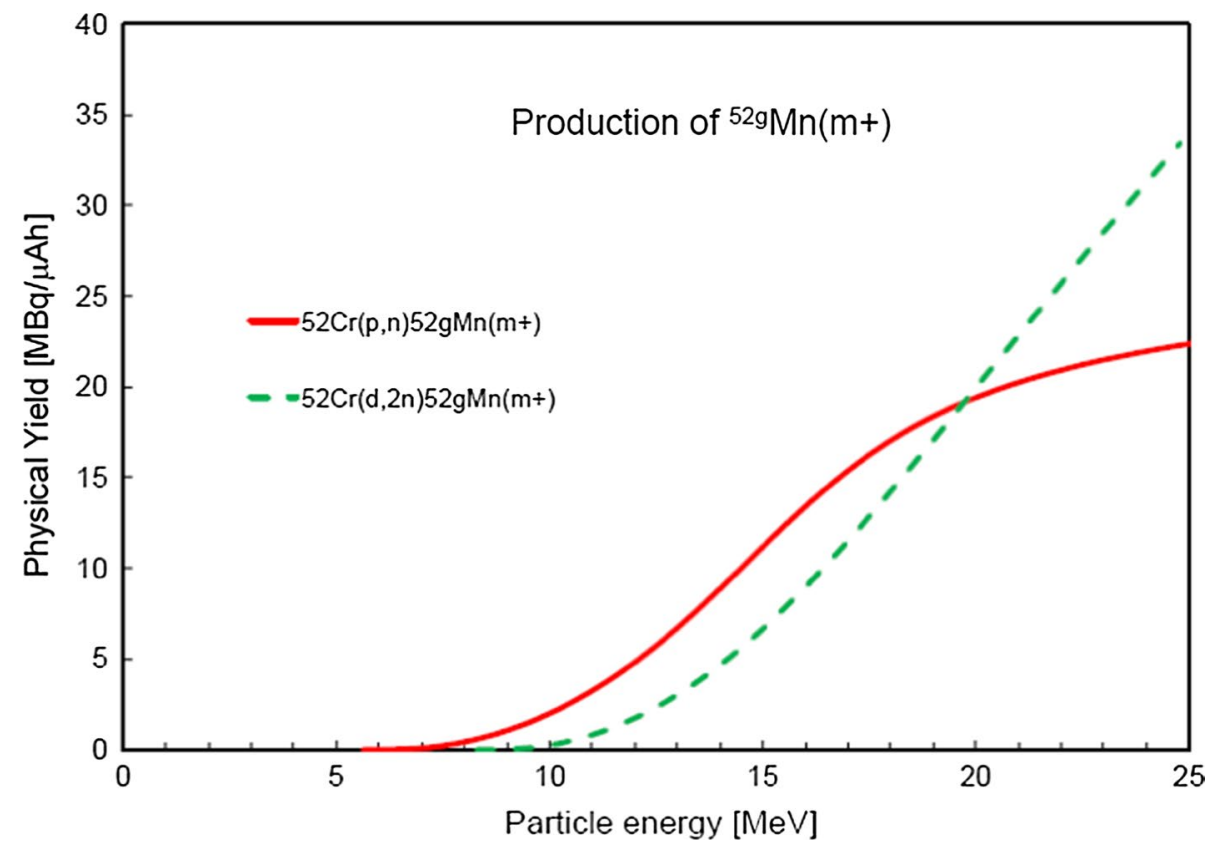




\section{Production of ${ }^{55} \mathrm{Co}\left(T_{1 / 2}=17.53 \mathrm{~h}\right)$}

Applications: ${ }^{55} \mathrm{Co}$ is a typical example of a positron emitter of sufficient half-life to follow kinetic processes that function over a longer timescale. This radionuclide has been used to target the epidermal growth factor (EGFR) by means of labelled DOTA-conjugated Affibody. Exhibits lower liver and heart uptake for metal-chelate peptide complexes, with improved performance when compared with ${ }^{68} \mathrm{Ga}$. Also used as a $\mathrm{Ca}^{2+}$ analogue in imaging studies of Alzheimer disease, and shows promise in achieving improved imaging of cancer diseases.

${ }^{55} \mathrm{Co}(17.53 \mathrm{~h}): \beta^{+}(76 \%)$, and $E_{\gamma}(\mathrm{keV})\left(P_{\gamma}(\%)\right): 931.1(75)$, $1316.6(7.1)$.
${ }^{58} \mathrm{Ni}(\mathrm{p}, \alpha){ }^{55} \mathrm{Co},{ }^{54} \mathrm{Fe}(\mathrm{d}, \mathrm{n}){ }^{55} \mathrm{Co}$ and ${ }^{56} \mathrm{Fe}(\mathrm{p}, 2 \mathrm{n}){ }^{55} \mathrm{Co}$ production routes have been evaluated.

\section{${ }^{58} \mathrm{Ni}(\mathrm{p}, \mathrm{a})^{55} \mathrm{Co}$}

The seventeen experimental datasets available in the literature are shown in Fig. 30 [36, 45, 59, 74-87] together with the TENDL calculations. One dataset was rejected (Haasbroek et al. [76], values too high), while the remaining sixteen datasets were used in the statistical fitting procedure. The selected data and their experimental uncertainties are shown in Fig. 31 together with the Padé fit ( $L=10, N=352$, $\left.\chi^{2}=1.97\right)$ and estimated uncertainty in percentages, including $4 \%$ systematic uncertainty (right-hand scale).
Fig. 30 Seventeen experimental datasets for the ${ }^{58} \mathrm{Ni}(\mathrm{p}, \alpha)^{55} \mathrm{Co}$ reaction available in the literature [36, 45, 59, 74-87], and TENDL calculations

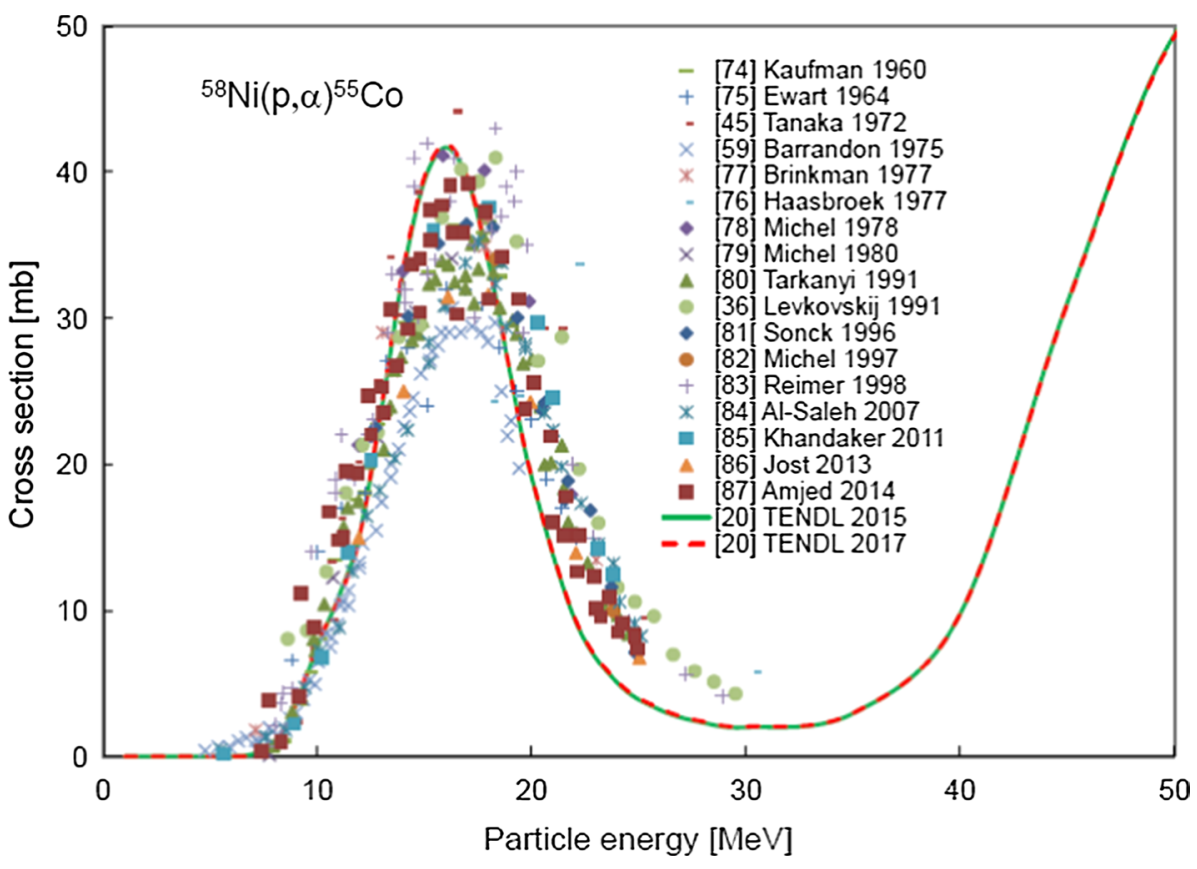


Fig. 31 Sixteen selected experimental datasets for the ${ }^{58} \mathrm{Ni}(\mathrm{p}, \alpha)^{55} \mathrm{Co}$ reaction $[36,45$, $59,74,75,77-87]$ with the Padé fit $\left(L=10, N=352, \chi^{2}=1.97\right.$, solid line) and estimated total uncertainties in percentages, including $4 \%$ systematic uncertainty (dashed line, right-hand scale)

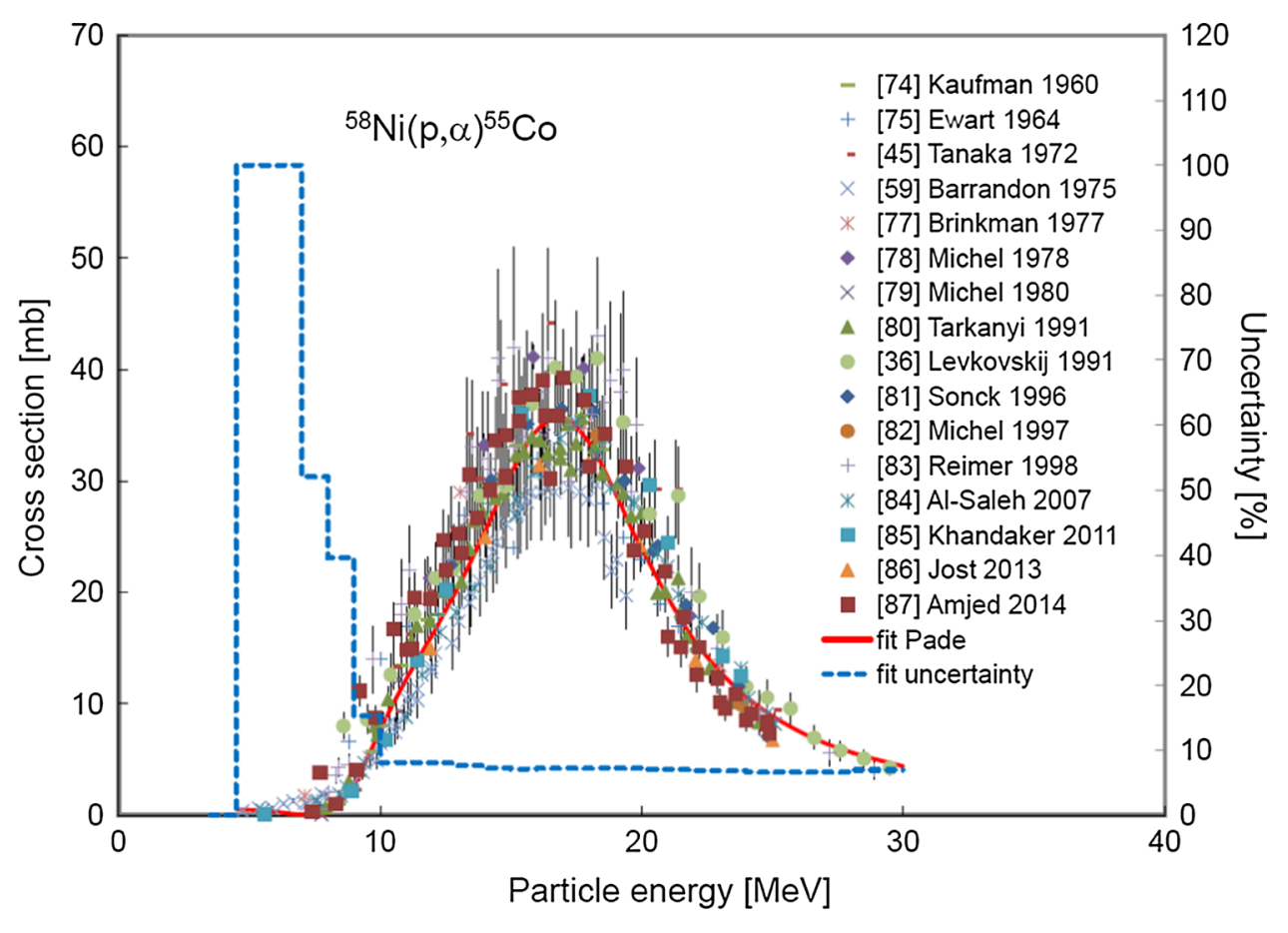




\section{${ }^{54} \mathrm{Fe}(\mathrm{d}, \mathrm{n}){ }^{55} \mathrm{Co}$}

The ten experimental datasets available in the literature are shown in Fig. 32 [88-97] together with the TENDL calculations. One dataset was rejected (Clark et al. [89], values too high), while the remaining nine datasets were used in the statistical fitting procedure (although some very discrepant points around $10 \mathrm{MeV}$ from Hermanne [94] and the highest three points from Zhenlan [91] were also discarded). The selected data and their experimental uncertainties are shown in Fig. 33 together with the Padé fit ( $\left.L=13, N=170, \chi^{2}=2.14\right)$ and estimated uncertainty in percentages, including $4 \%$ systematic uncertainty (right-hand scale).
Fig. 32 Ten experimental datasets for the ${ }^{54} \mathrm{Fe}(\mathrm{d}, \mathrm{n}){ }^{55} \mathrm{Co}$ reaction available in the literature [88-97], and TENDL calculations
Fig. 33 Nine selected experimental datasets for the ${ }^{54} \mathrm{Fe}(\mathrm{d}, \mathrm{n}){ }^{55} \mathrm{Co}$ reaction $[88$, 90-97] with the Padé fit $(L=13$, $N=170, \chi^{2}=2.14$, solid line) and estimated total uncertainties in percentages, including $4 \%$ systematic uncertainty (dashed line, right-hand scale)
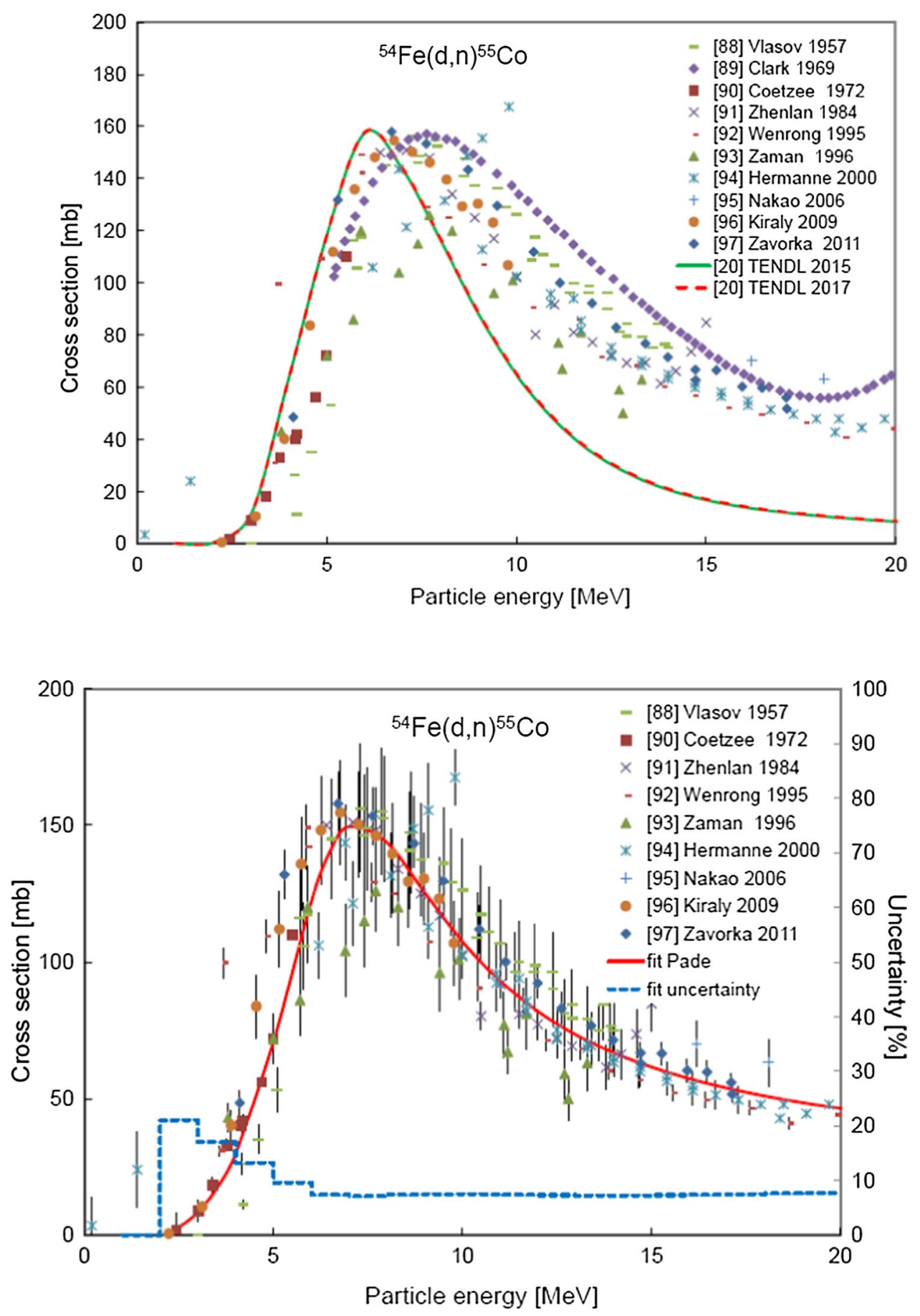


\section{${ }^{56} \mathrm{Fe}(\mathrm{p}, 2 \mathrm{n}){ }^{55} \mathrm{Co}$}

The fifteen experimental datasets available in the literature are shown in Fig. 34 [36, 59, 82, 98-109] together with the TENDL calculations. Four datasets were rejected (Michel et al. [82], Cohen and Newman [98], Williams and Fulmer [99], and Ditrói et al. [107], all show discrepant values), and the remaining eleven sets were used in the statistical fitting procedure. The selected data and their experimental uncertainties are shown in Fig. 35 together with the Padé fit $(L=8$, $\left.N=101, \chi^{2}=2.74\right)$ and estimated uncertainty in percentages, including $4 \%$ systematic uncertainty (right-hand scale).
Fig. 34 Fifteen experimental datasets for the ${ }^{56} \mathrm{Fe}(\mathrm{p}, 2 \mathrm{n}){ }^{55} \mathrm{Co}$ reaction available in the literature [36, 59, 82, 98-109], and TENDL calculations

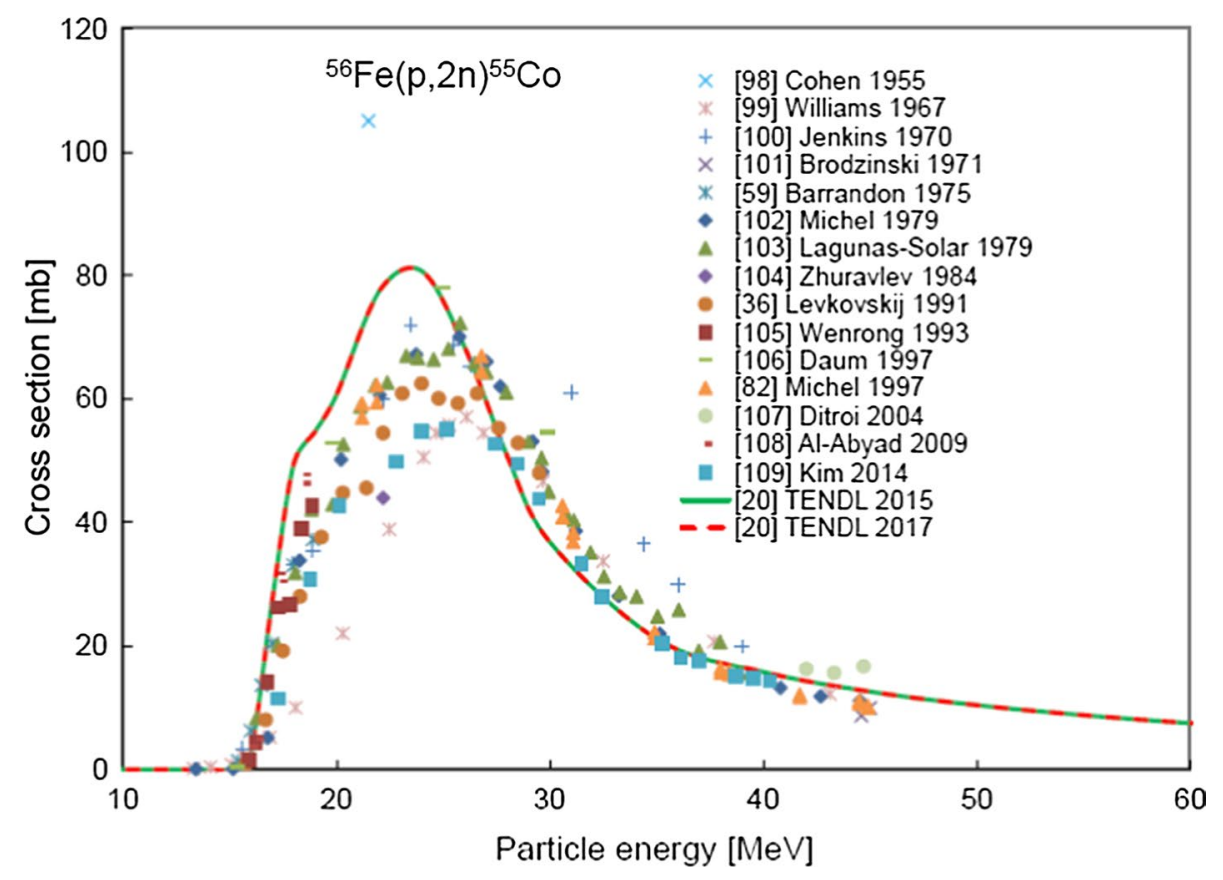

Fig. 35 Eleven selected experimental datasets for the ${ }^{56} \mathrm{Fe}(\mathrm{p}, 2 \mathrm{n}){ }^{55} \mathrm{Co}$ reaction $[36$, 59, 100-106, 108, 109] with the Padé fit $(L=8, N=101$, $\chi^{2}=2.74$, solid line) and estimated total uncertainties in percentages, including $4 \%$ systematic uncertainty (dashed line, right-hand scale)

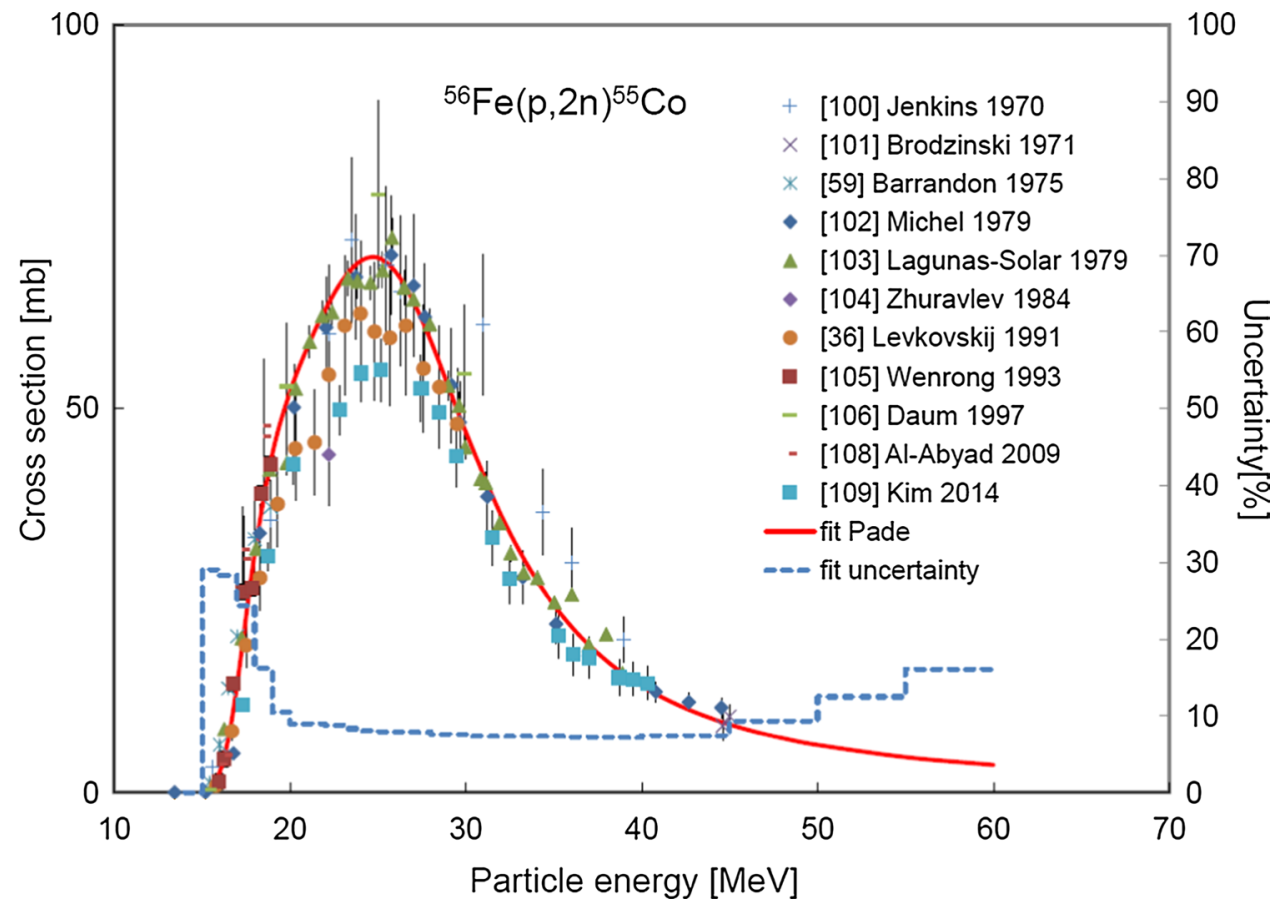


Thick target yields for production of ${ }^{55} \mathrm{Co}$

See Fig. 36.

Fig. 36 Thick target yields calculated from the recommended cross sections for the ${ }^{58} \mathrm{Ni}(\mathrm{p}, \alpha){ }^{55} \mathrm{Co},{ }^{54} \mathrm{Fe}(\mathrm{d}, \mathrm{n}){ }^{55} \mathrm{Co}$ and ${ }^{56} \mathrm{Fe}(\mathrm{p}, 2 \mathrm{n}){ }^{55} \mathrm{Co}$ reactions

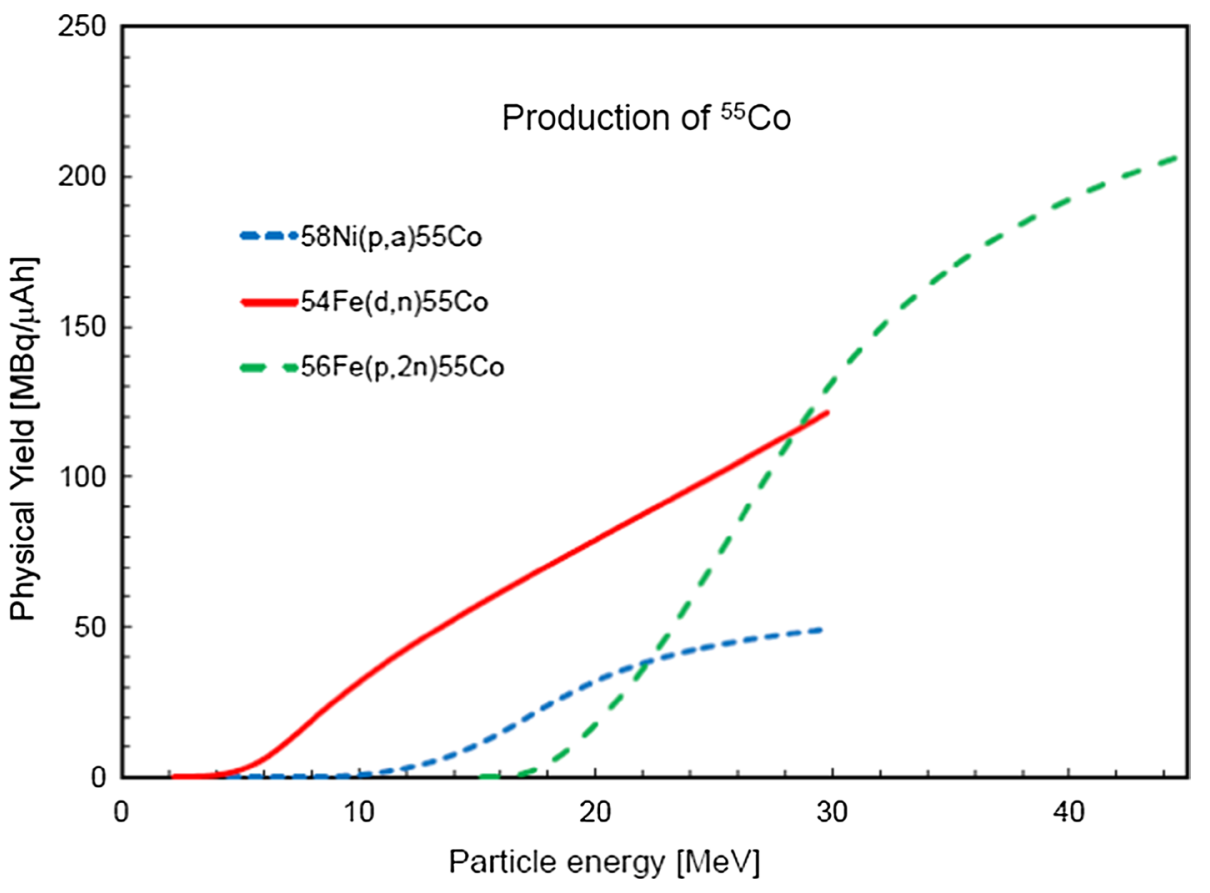




\section{Production of ${ }^{61} \mathrm{Cu}\left(T_{1 / 2}=3.339 \mathrm{~h}\right)$}

Applications: Copper radionuclides form stable complexes with several chelators that can be conjugated to a wide variety of organic molecules for both imaging $\left({ }^{61} \mathrm{Cu},{ }^{62} \mathrm{Cu},{ }^{64} \mathrm{Cu}\right)$ and radiotherapy $\left({ }^{64} \mathrm{Cu},{ }^{67} \mathrm{Ci}\right)$. Relatively longer-lived ${ }^{61} \mathrm{Cu}$ $\left(T_{1 / 2}=3.339 \mathrm{~h}, 61 \% \beta^{+}, 39 \% \mathrm{EC}\right)$ possesses very good imaging properties that can be used for blood flow studies in a similar manner to ${ }^{51} \mathrm{Cr}$. Also has been applied to blood pool imaging (DOTA-human serum albumin) and the study of hypoxia in tumours (coupled to ATSM) - useful for following kinetics processes of the order of a few hours.

${ }^{61} \mathrm{Cu}(3.339 \mathrm{~h}): \beta^{+}(61 \%)$, and $E_{\gamma}(\mathrm{keV})\left(P_{\gamma}(\%)\right): 282.956$ (12.2), 656.008 (10.8), 1185.234 (3.7).

Evaluations have been made of the ${ }^{61} \mathrm{Ni}(\mathrm{p}, \mathrm{n}){ }^{61} \mathrm{Cu}$, ${ }^{60} \mathrm{Ni}(\mathrm{d}, \mathrm{n}){ }^{61} \mathrm{Cu}$ and ${ }^{64} \mathrm{Zn}(\mathrm{p}, \alpha){ }^{61} \mathrm{Cu}$ direct production routes.
${ }^{61} \mathrm{Ni}(p, n){ }^{61} \mathrm{Cu}$

The seventeen experimental datasets available in the literature are shown in Fig. 37 [45, 56, 59, 64, 78, 79, 84, 87, 110-117] together with the TENDL calculations. Ref. [112] contains two datasets, labelled (a) and (b). Five datasets were rejected (Blosser and Handley [56], Tanaka and Furukawa [64], Barrandon et al. [59], Michel et al. [78], and Al-Saleh et al. [84], all of these datasets exhibit maximum values that are too high), and the remaining twelve sets were used in the statistical fitting procedure. The selected data and their experimental uncertainties are shown in Fig. 38 together with the Padé fit $\left(L=12, N=192, \chi^{2}=2.81\right)$ and estimated uncertainty in percentages, including $4 \%$ systematic uncertainty (right-hand scale).
Fig. 37 Seventeen experimental datasets for the ${ }^{61} \mathrm{Ni}(\mathrm{p}, \mathrm{n})^{61} \mathrm{Cu}$ reaction available in the literature $[45,56,59,64,78,79$, 84, 87, 110-117], and TENDL calculations

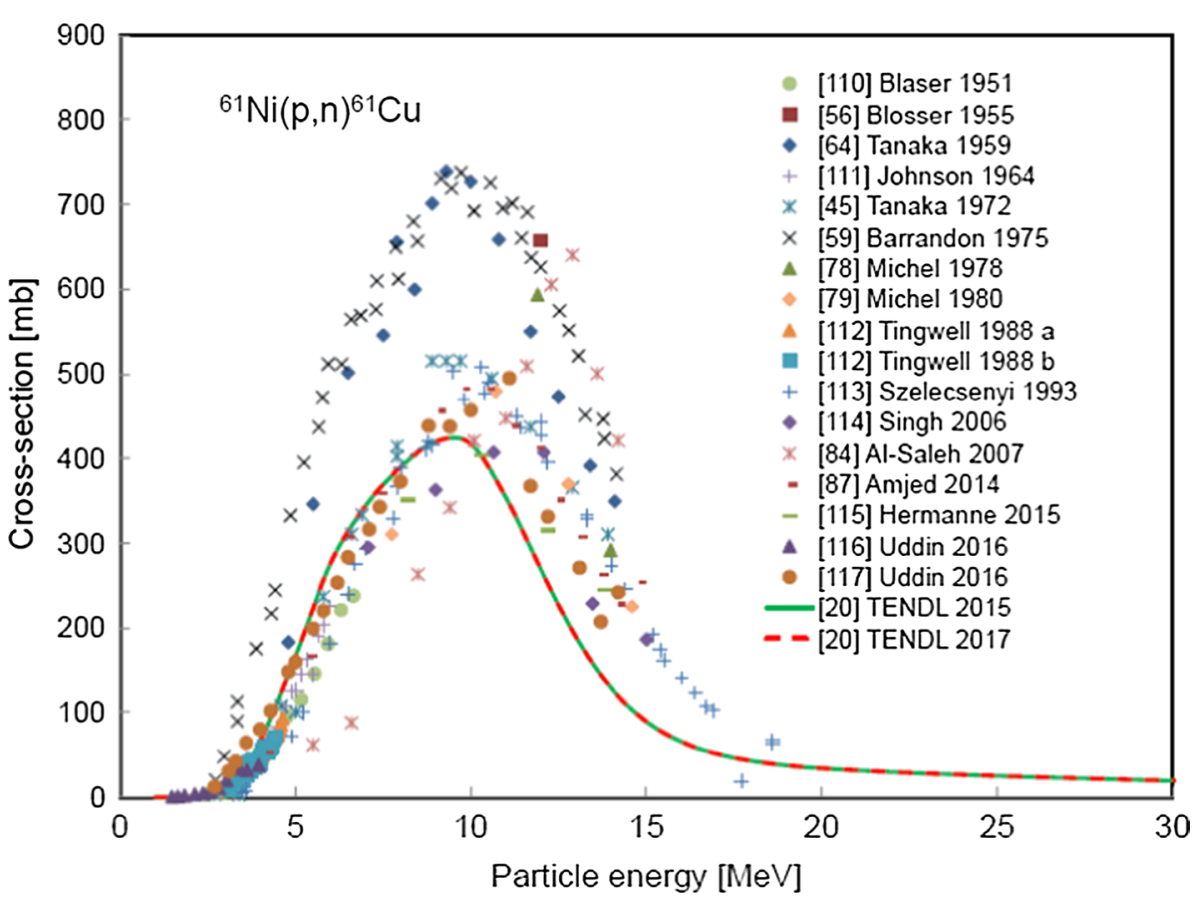


Fig. 38 Twelve selected experimental datasets for the ${ }^{61} \mathrm{Ni}(\mathrm{p}, \mathrm{n}){ }^{61} \mathrm{Cu}$ reaction $[45,79$, 87, 110-117] with the Padé fit $\left(L=12, N=192, \chi^{2}=2.81\right.$, solid line) and estimated total uncertainties in percentages, including $4 \%$ systematic uncertainty (dashed line, right-hand scale)

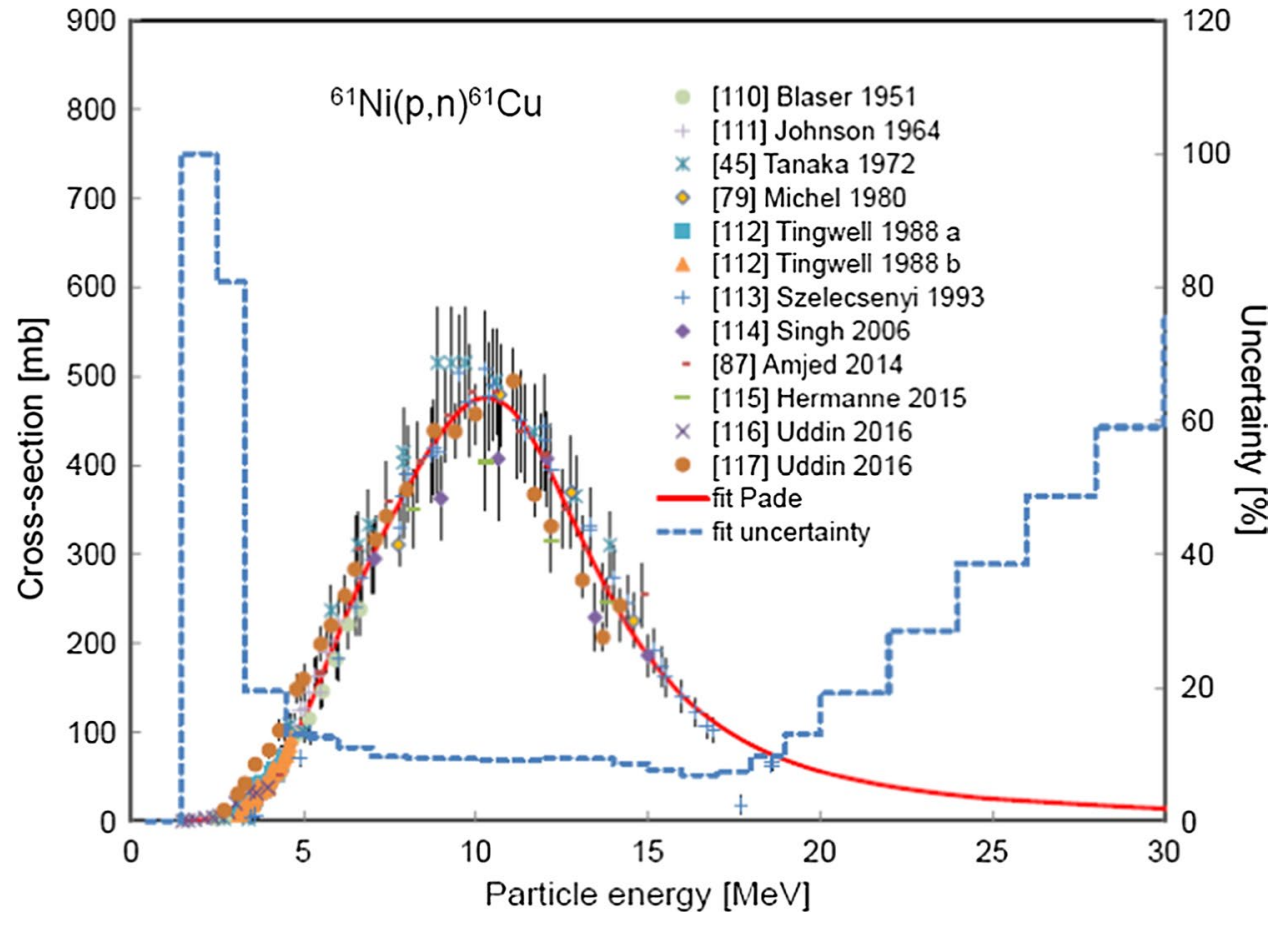




\section{${ }^{60} \mathrm{Ni}(\mathrm{d}, \mathrm{n})^{61} \mathrm{Cu}$}

The five experimental datasets available in the literature are shown in Fig. 39 [90, 118-121] together with the TENDL calculations. All datasets were used in the statistical fitting procedure (Cogneau et al. [118] data were normalised, and data above $6-\mathrm{MeV}$ particle beam energy discarded as inconsistent with model calculations). All of the data and their experimental uncertainties are shown in Fig. 40 together with the Pade fit $\left(L=16, N=29, \chi^{2}=1.16\right)$ and estimated uncertainty in percentages, including $4 \%$ systematic uncertainty (right-hand scale). This reaction is the main contributor to the formation of ${ }^{61} \mathrm{Cu}$ on natural $\mathrm{Ni}$ by deuterons, adopted as a suitable beam monitor (see Ref. [30], Sect. 3.I).
Fig. 39 Five experimental datasets for the ${ }^{60} \mathrm{Ni}(\mathrm{d}, \mathrm{n}){ }^{61} \mathrm{Cu}$ reaction available in the literature [90, 118-121], and TENDL calculations

Fig. 40 Five experimental datasets for the ${ }^{60} \mathrm{Ni}(\mathrm{d}, \mathrm{n}){ }^{61} \mathrm{Cu}$ reaction [90, 118-121] with the Padé fit $(L=16, N=29$, $\chi^{2}=1.16$, solid line) and estimated total uncertainties in percentages, including $4 \%$ systematic uncertainty (dashed line, right-hand scale)
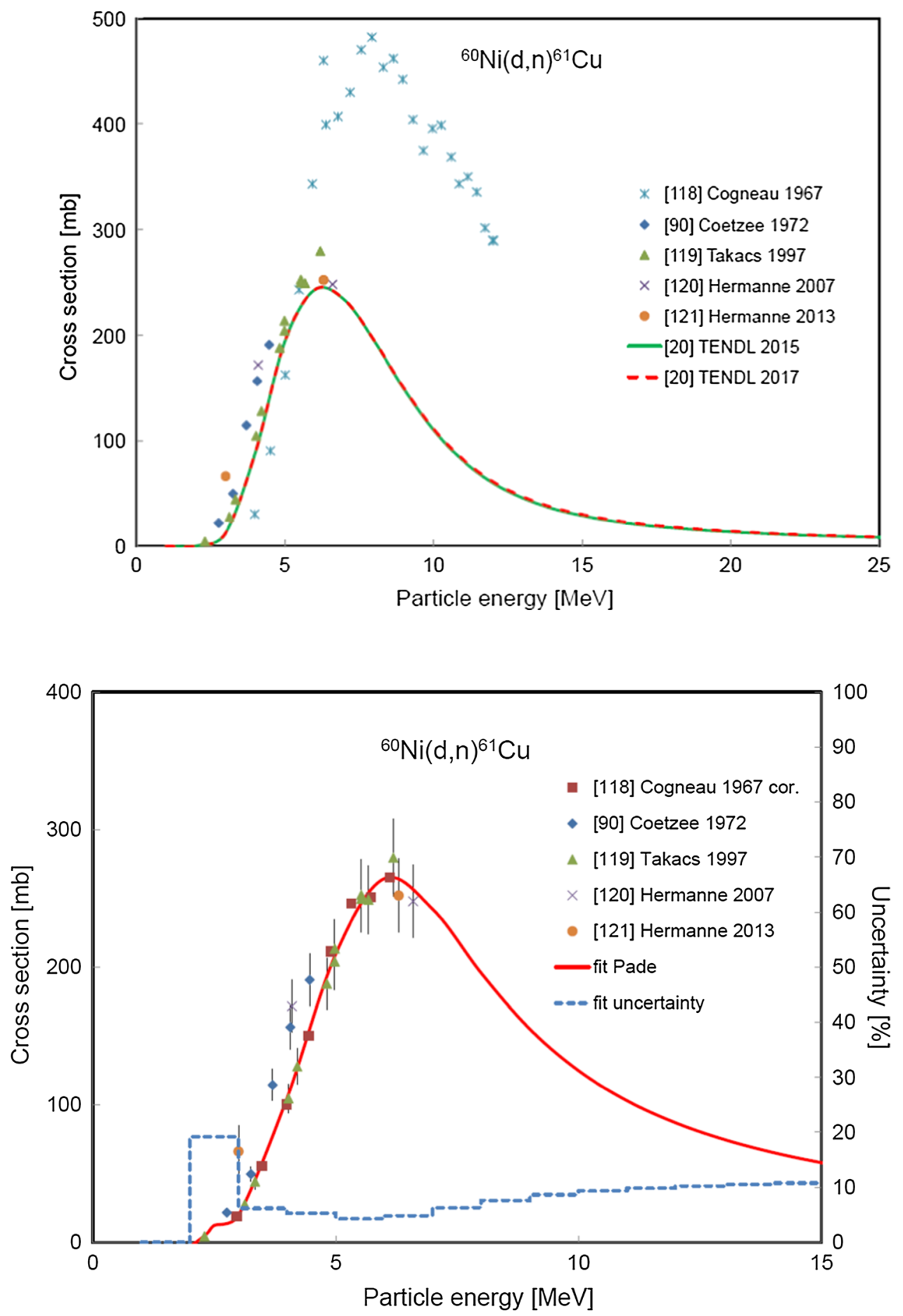
${ }^{64} \mathrm{Zn}(\mathrm{p}, \mathrm{a}){ }^{61} \mathrm{Cu}$

The seven experimental datasets available in the literature are shown in Fig. 41 [36, 59, 122-126] together with the TENDL calculations. One dataset was rejected (Barrandon et al. [59], discrepant behaviour near maximum), and the remaining six sets were used in the statistical fitting procedure. The selected data and their experimental uncertainties are shown in Fig. 42 together with the Padé $(L=12, N=72$, $\left.\chi^{2}=0.88\right)$ and estimated uncertainty in percentages, including $4 \%$ systematic uncertainty (right-hand scale).
Fig. 41 Seven experimental datasets for the ${ }^{64} \mathrm{Zn}(\mathrm{p}, \alpha){ }^{61} \mathrm{Cu}$ reaction available in the literature [36, 59, 122-126], and TENDL calculations
Fig. 42 Six selected experimental datasets $[36,122-126]$ for the ${ }^{64} \mathrm{Zn}(\mathrm{p}, \alpha){ }^{61} \mathrm{Cu}$ reaction with the Padé fit $(L=12$, $N=72, \chi^{2}=0.88$, solid line) and estimated total uncertainties in percentages, including $4 \%$ systematic uncertainty (dashed line, right-hand scale)
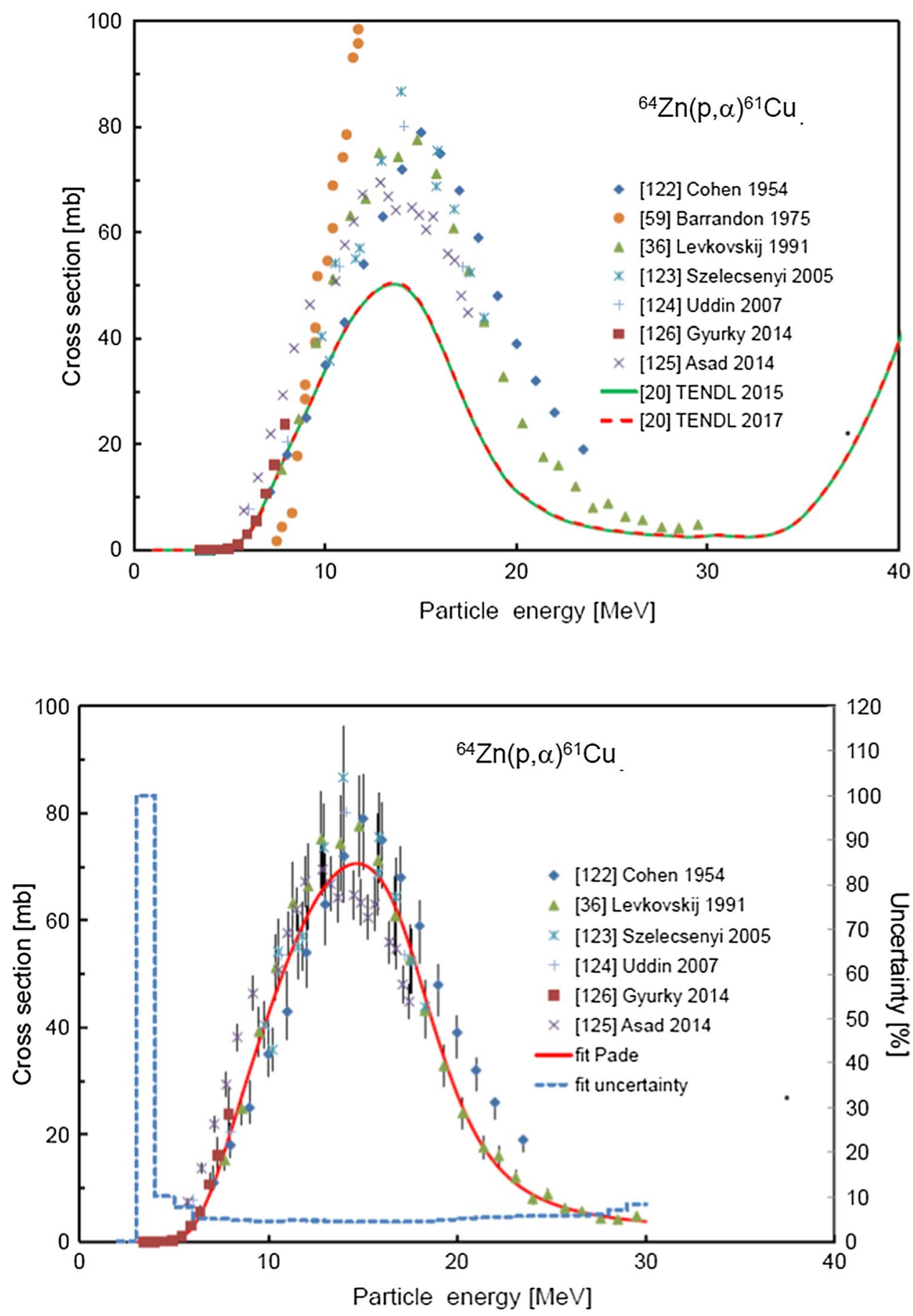
Thick target yields for production of ${ }^{61} \mathrm{Cu}$

See Fig. 43.

Fig. 43 Thick target yields calculated from the recommended cross sections for the ${ }^{61} \mathrm{Ni}(\mathrm{p}, \mathrm{n}){ }^{61} \mathrm{Cu},{ }^{60} \mathrm{Ni}(\mathrm{d}, \mathrm{n}){ }^{61} \mathrm{Cu}$ and ${ }^{64} \mathrm{Zn}(\mathrm{p}, \alpha)^{61} \mathrm{Cu}$ reactions

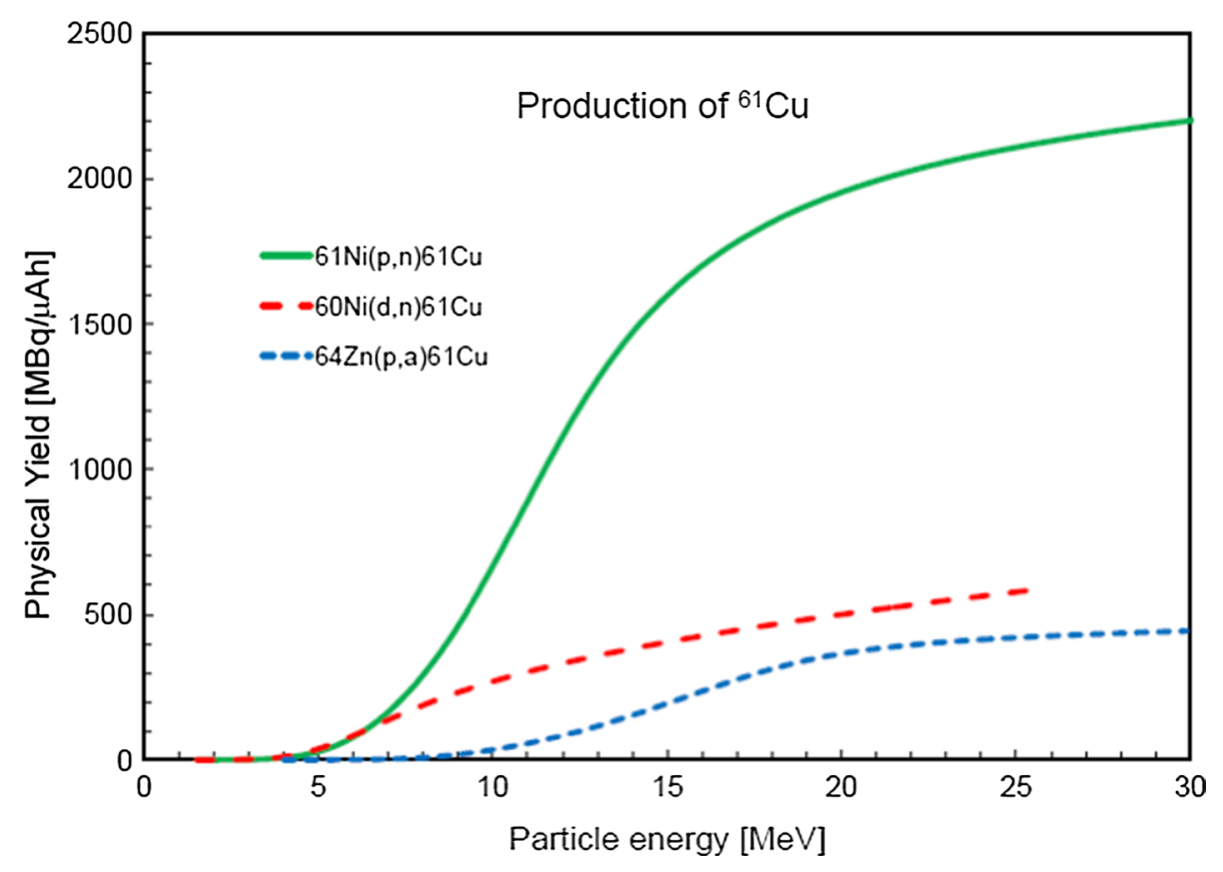




\section{Production of ${ }^{62} \mathrm{Cu}\left(T_{1 / 2}=9.67 \mathrm{~min}\right)$ and longer-lived \\ ${ }^{62} \mathrm{Zn}$ parent $\left(T_{1 / 2}=9.193 \mathrm{~h}\right)$}

Applications: As stated earlier, copper isotopes form stable complexes with several chelators that can be conjugated to a wide variety of organic molecules for both imaging $\left({ }^{61} \mathrm{Cu}\right.$, $\left.{ }^{62} \mathrm{Cu},{ }^{64} \mathrm{Cu}\right)$ and therapy $\left({ }^{64} \mathrm{Cu},{ }^{67} \mathrm{Ci}\right)$. Short-lived ${ }^{62} \mathrm{Cu}$ has been proposed for the labelling of PTSM (pyruvaldehyde bis) to undertake myocardial and brain blood flow studies.

${ }^{62} \mathrm{Cu}(9.67 \mathrm{~min}): \beta^{+}(97.83 \%)$, and $E_{\gamma}(\mathrm{keV})\left(P_{\gamma}(\%)\right): 875.66$ (0.147), 1172.97 (0.342).

${ }^{62} \mathrm{Zn}(9.193 \mathrm{~h}): \beta^{+}(8.2 \%)$, and $E_{\gamma}(\mathrm{keV})\left(P_{\gamma}(\%)\right): 548.35$ (15.3), 596.56 (26).

Evaluations have been made of the ${ }^{63} \mathrm{Cu}(\mathrm{p}, 2 \mathrm{n}){ }^{62} \mathrm{Zn}$, ${ }^{63} \mathrm{Cu}(\mathrm{d}, 3 \mathrm{n}){ }^{62} \mathrm{Zn}$ and ${ }^{\mathrm{nat}} \mathrm{Ni}(\alpha, \mathrm{xn}){ }^{62} \mathrm{Zn}$ indirect, and ${ }^{62} \mathrm{Ni}(\mathrm{p}, \mathrm{n}){ }^{62} \mathrm{Cu}$ and ${ }^{62} \mathrm{Ni}(\mathrm{d}, 2 \mathrm{n}){ }^{62} \mathrm{Cu}$ direct production routes.
${ }^{63} \mathrm{Cu}(\mathrm{p}, 2 \mathrm{n}){ }^{62} \mathrm{Zn}$

Twenty-four experimental datasets available in the literature are shown in Fig. 44 [36, 82, 98, 99, 127-146] together with the TENDL calculations. Seven datasets were rejected (Ghoshal [127], Williams and Fulmer [99], Greene and Lebowitz [128], Greenwood and Smither [130], Aleksandrov et al. [132], Levkovskij [36], and Tárkányi et al. [142], all disagree significantly with the other datasets), and the remaining seventeen sets were used in the statistical fitting procedure. The selected data and their experimental uncertainties are shown in Fig. 45 together with the Padé fit $(L=16, N=213$, $\chi^{2}=1.89$ ) and estimated uncertainty in percentages, including $4 \%$ systematic uncertainty (right-hand scale). As the only reaction known to contribute to the formation of ${ }^{62} \mathrm{Zn}$ on ${ }^{\text {nat }} \mathrm{Cu}$ for protons below $30 \mathrm{MeV}$, the fitted data have been adopted as a beam monitor in this energy region (see Ref. [30], Sect. 2.6).
Fig. 44 Twenty-four experimental datasets for the

${ }^{63} \mathrm{Cu}(\mathrm{p}, 2 \mathrm{n}){ }^{62} \mathrm{Zn}$ reaction available in the literature $[36,82$, 98, 99, 127-146], and TENDL calculations

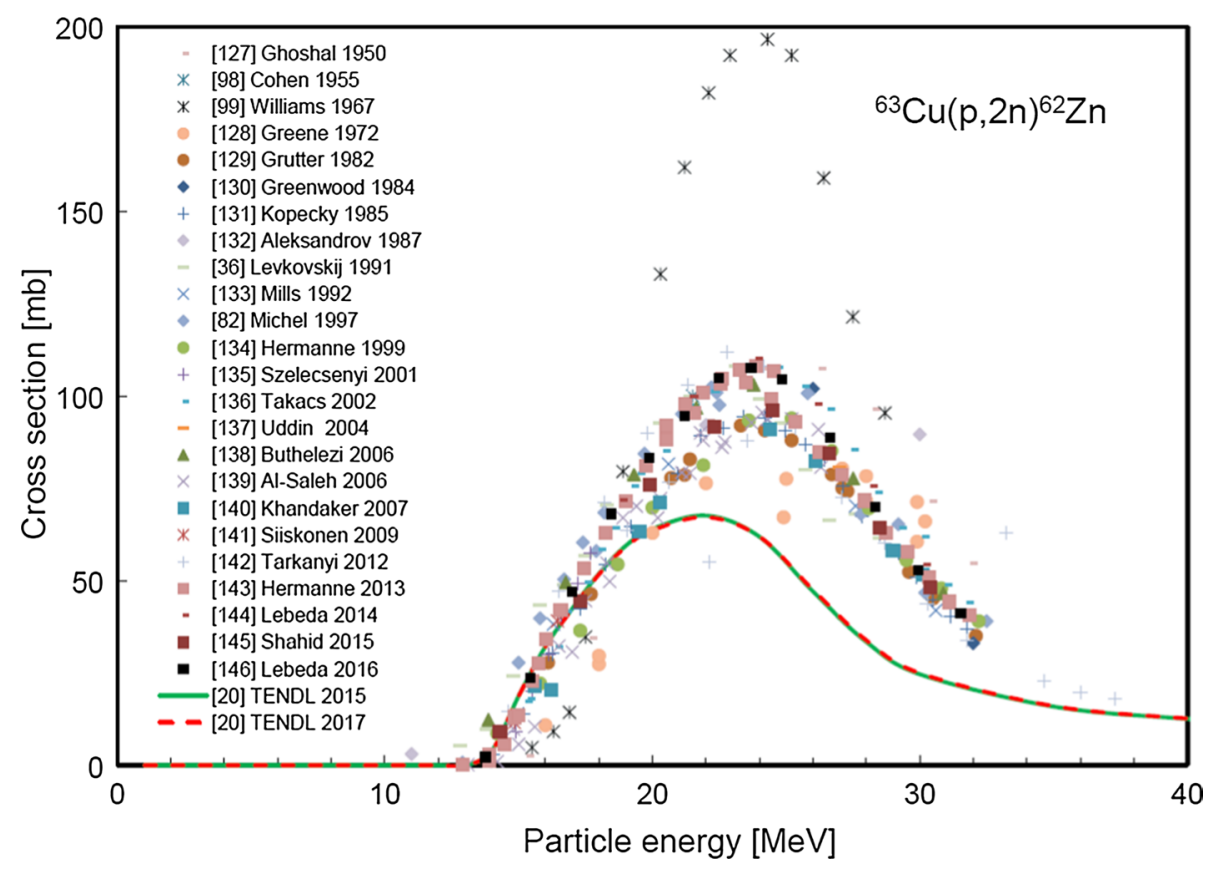


Fig. 45 Seventeen selected experimental datasets for the ${ }^{63} \mathrm{Cu}(\mathrm{p}, 2 \mathrm{n}){ }^{62} \mathrm{Zn}$ reaction $[82,98$, $129,131,133-141,143-146]$ with the Padé fit $(L=16$, $N=213, \chi^{2}=1.89$, solid line) and estimated total uncertainties in percentages, including $4 \%$ systematic uncertainty (dashed line, right-hand scale)

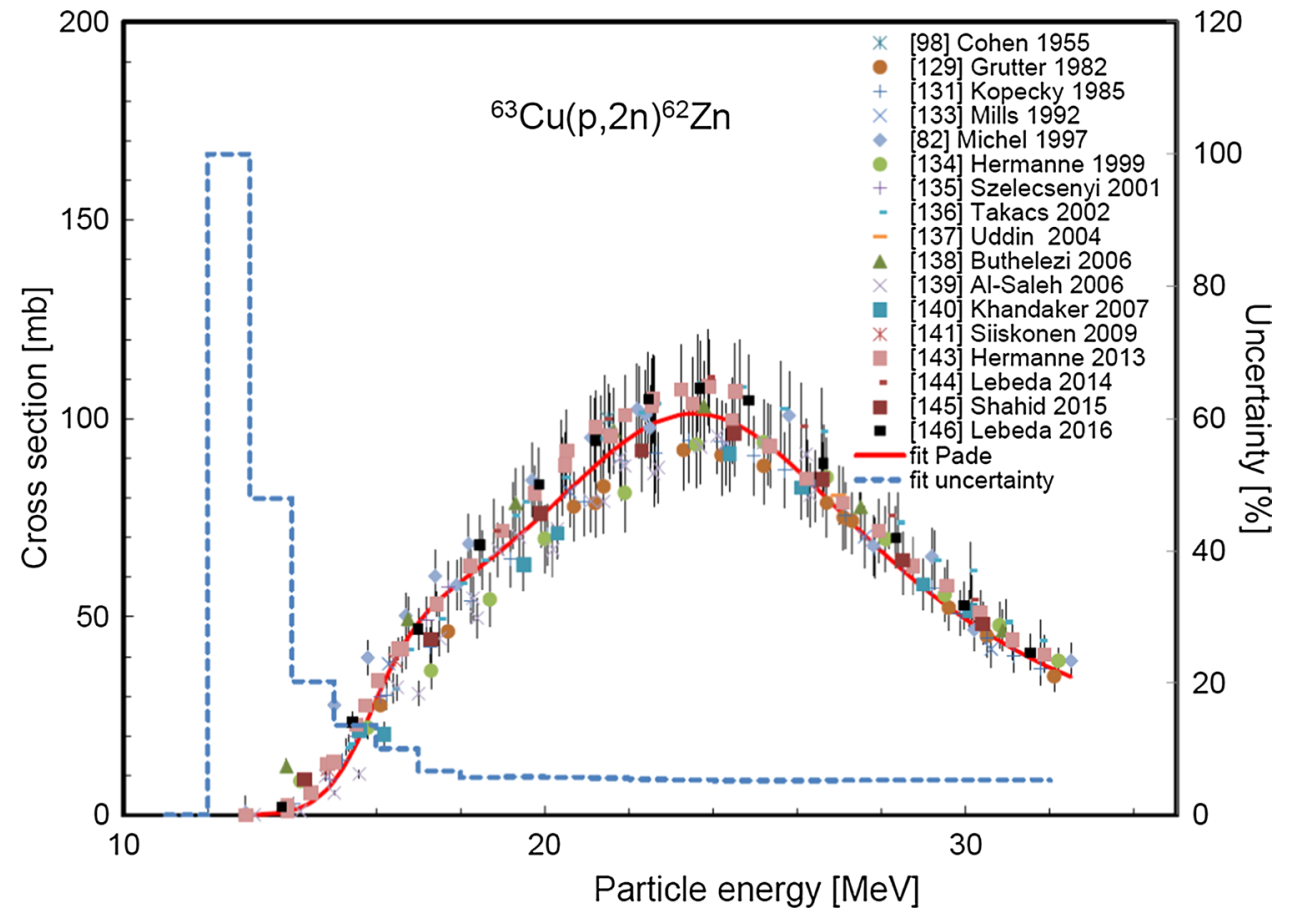




\section{${ }^{63} \mathrm{Cu}(\mathrm{d}, 3 \mathrm{n}){ }^{62} \mathrm{Zn}$}

While a known dataset by Bartell et al. [147] is not represented in Fig. 46 because the values are totally discrepant even after arbitrary normalisation, eight other experimental datasets available in the literature are shown [73, 95, 148-153] together with the TENDL calculations. The data by Fulmer and Williams [148] were subsequently rejected because they disagree significantly with the other datasets (attributed to the normalisation of inadequately defined low-intensity decay data). All of the remaining seven datasets were used in the statistical fitting procedure. The selected data and their experimental uncertainties are shown in Fig. 47 together with the Padé fit $(L=12, N=82$, $\chi^{2}=1.89$ ) and estimated uncertainty in percentages, including $4 \%$ systematic uncertainty (right-hand scale). As the only reaction known to contribute to the formation of ${ }^{62} \mathrm{Zn}$ on ${ }^{\text {nat }} \mathrm{Cu}$ for deuterons below $35 \mathrm{MeV}$, the fitted data have been adopted as a beam monitor in this energy region (see Ref. [30], Section III E).
Fig. 46 Eight experimental datasets for the ${ }^{63} \mathrm{Cu}(\mathrm{d}, 3 \mathrm{n})^{62} \mathrm{Zn}$ reaction available in the literature [73, 95, 148-153], and TENDL calculations
Fig. 47 Seven selected experimental datasets for the ${ }^{63} \mathrm{Cu}(\mathrm{d}, 3 \mathrm{n}){ }^{62} \mathrm{Zn}$ reaction $[73$, $95,149-153]$ with the Padé fit $\left(L=12, N=82, \chi^{2}=1.89\right.$, solid line) and estimated total uncertainties in percentages, including $4 \%$ systematic uncertainty (dashed line, right-hand scale)
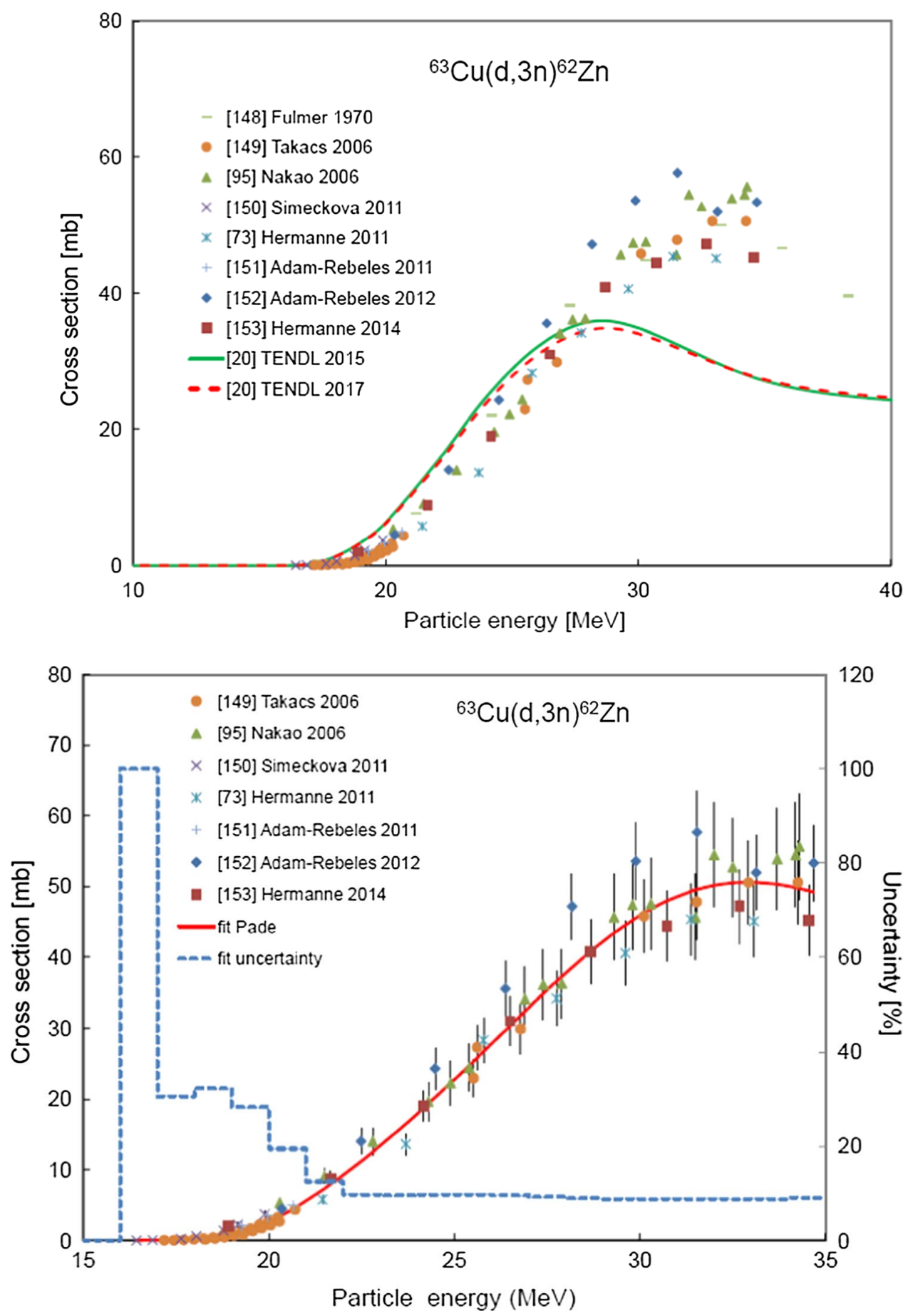


\section{${ }^{\text {nat }} \mathrm{Ni}(a, x n)^{62} \mathrm{Zn}$}

The nine experimental datasets available in the literature are shown in Fig. 48 [36, 127, 154-160] together with the TENDL calculations. Three datasets were rejected (Neirinckx [155] (energy shift near threshold), Singh et al. [159] (discrepant values at energies below $35 \mathrm{MeV}$ ), and Yadav et al. [160] (value too low)), and the remaining six sets were used in the statistical fitting procedure. The selected data and their experimental uncertainties are shown in Fig. 49 together with the Padé fit $\left(L=21, N=45, \chi^{2}=1.72\right)$ and estimated uncertainty in percentages, including $4 \%$ systematic uncertainty (right-hand scale).
Fig. 48 Nine experimental datasets for the ${ }^{\text {nat }} \mathrm{Ni}(\alpha, \mathrm{xn})^{62} \mathrm{Zn}$ reaction available in the literature [36, 127, 154-160], and TENDL calculations
Fig. 49 Six selected experimental datasets for the ${ }^{\text {nat }} \mathrm{Ni}(\alpha, \mathrm{xn}){ }^{62} \mathrm{Zn}$ reaction $[36$, $127,154,156-158]$ with the Padé fit $(L=10, N=45$, $\chi^{2}=1.74$, solid line) and estimated total uncertainties in percentages, including $4 \%$ systematic uncertainty (dashed line, right-hand scale)
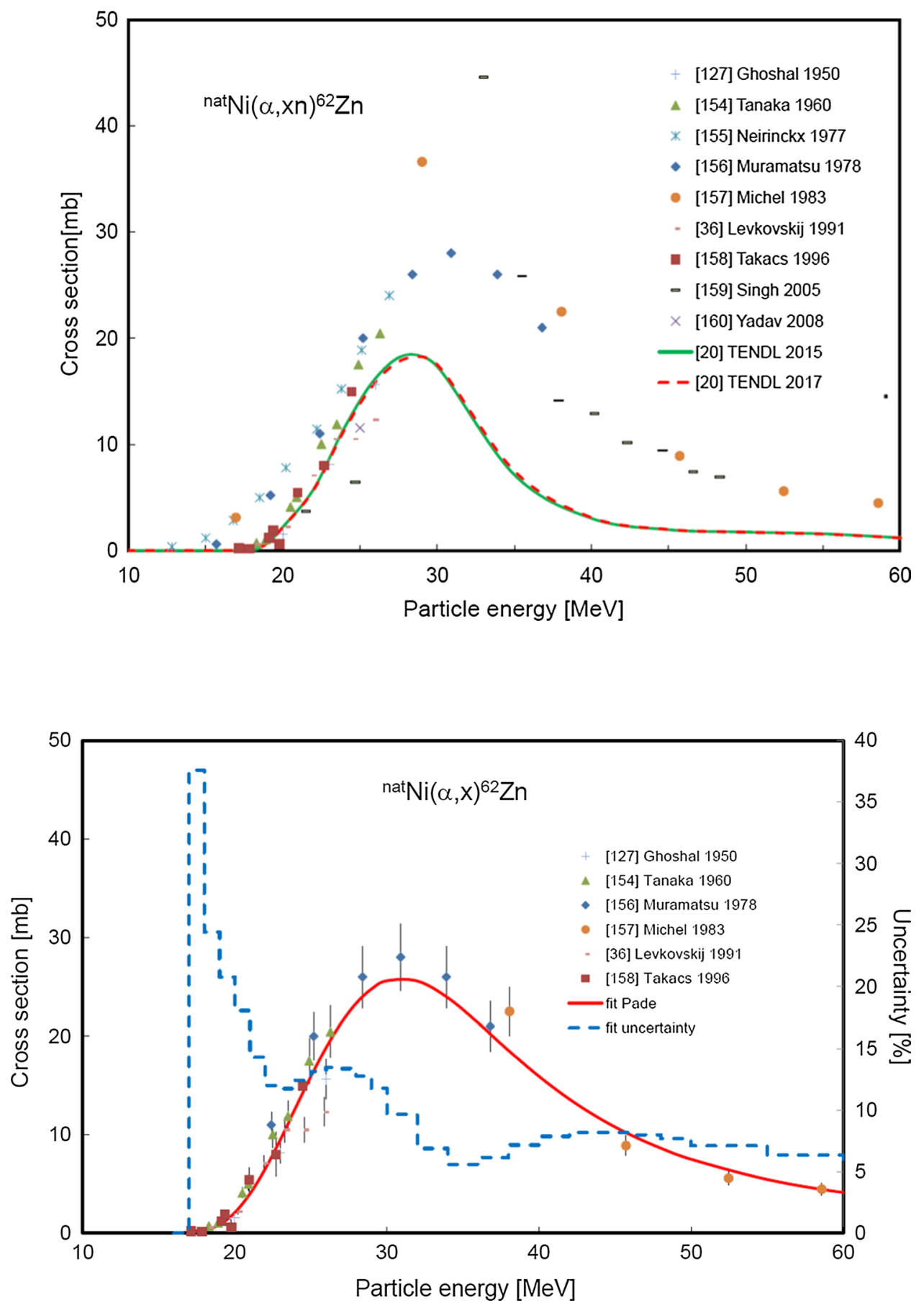
${ }^{62} \mathrm{Ni}(p, n){ }^{62} \mathrm{Cu}$

The seven experimental datasets available in the literature are shown in Fig. 50 [36, 45, 66, 110, 161-163] together with the TENDL calculations. All datasets were used in the statistical fitting procedure. The data and their experimental uncertainties are shown in Fig. 51 together with the Padé fit ( $L=12, N=77, \chi^{2}=1.33$ ) and estimated uncertainty in percentages, including $4 \%$ systematic uncertainty (right-hand scale).
Fig. 50 Seven experimental datasets for the ${ }^{62} \mathrm{Ni}(\mathrm{p}, \mathrm{n}){ }^{62} \mathrm{Cu}$ reaction available in the literature [36, 45, 66, 110, 161-163], and TENDL calculations
Fig. 51 Seven experimental datasets $[36,45,66,110,161-$ 163] for the ${ }^{62} \mathrm{Ni}(\mathrm{p}, \mathrm{n}){ }^{62} \mathrm{Cu}$ reaction with the Padé fit $(L=12$, $N=77, \chi^{2}=1.33$, solid line) and estimated total uncertainties in percentages, including $4 \%$ systematic uncertainty (dashed line, right-hand scale)
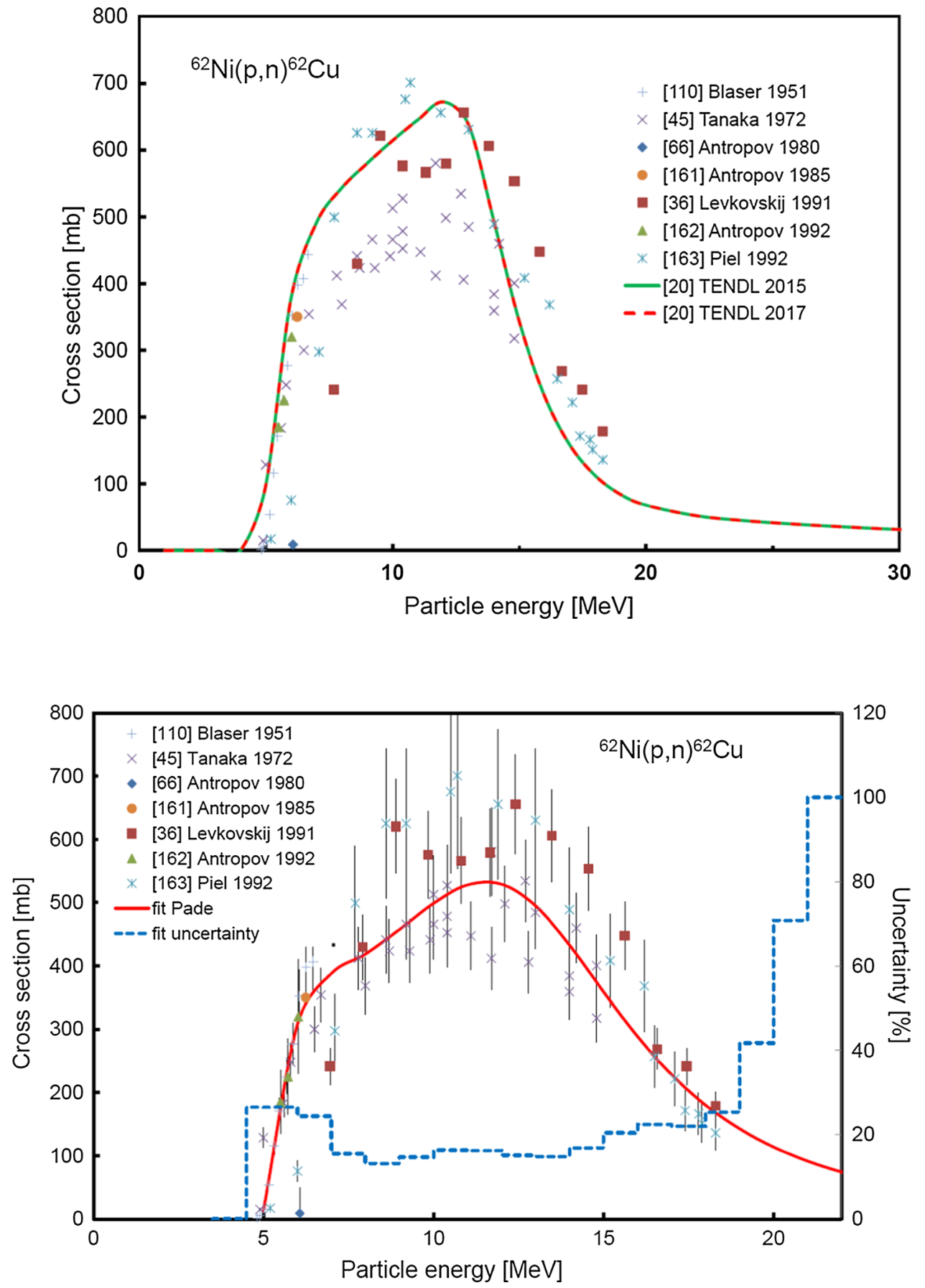


\section{${ }^{62} \mathrm{Ni}(d, 2 n){ }^{62} \mathrm{Cu}$}

The single dataset available in the literature is shown in Fig. 52 [118] together with the TENDL calculations. This dataset was used in the statistical fitting procedure. The data and their experimental uncertainties are shown in Fig. 53 together with the Padé fit $\left(L=5, N=16, \chi^{2}=0.83\right)$ and estimated uncertainty in percentages, including $4 \%$ systematic uncertainty (right-hand scale).
Fig. 52 Experimental dataset for the ${ }^{62} \mathrm{Ni}(\mathrm{d}, 2 \mathrm{n}){ }^{62} \mathrm{Cu}$ reaction available in the literature [118], and TENDL calculations
Fig. 53 Experimental dataset for the ${ }^{62} \mathrm{Ni}(\mathrm{d}, 2 \mathrm{n}){ }^{62} \mathrm{Cu}$ reaction [118] with the Padé fit $(L=5$, $N=16, \chi^{2}=0.83$, solid line) and estimated total uncertainties in percentages, including $4 \%$ systematic uncertainty (dashed line, right-hand scale)
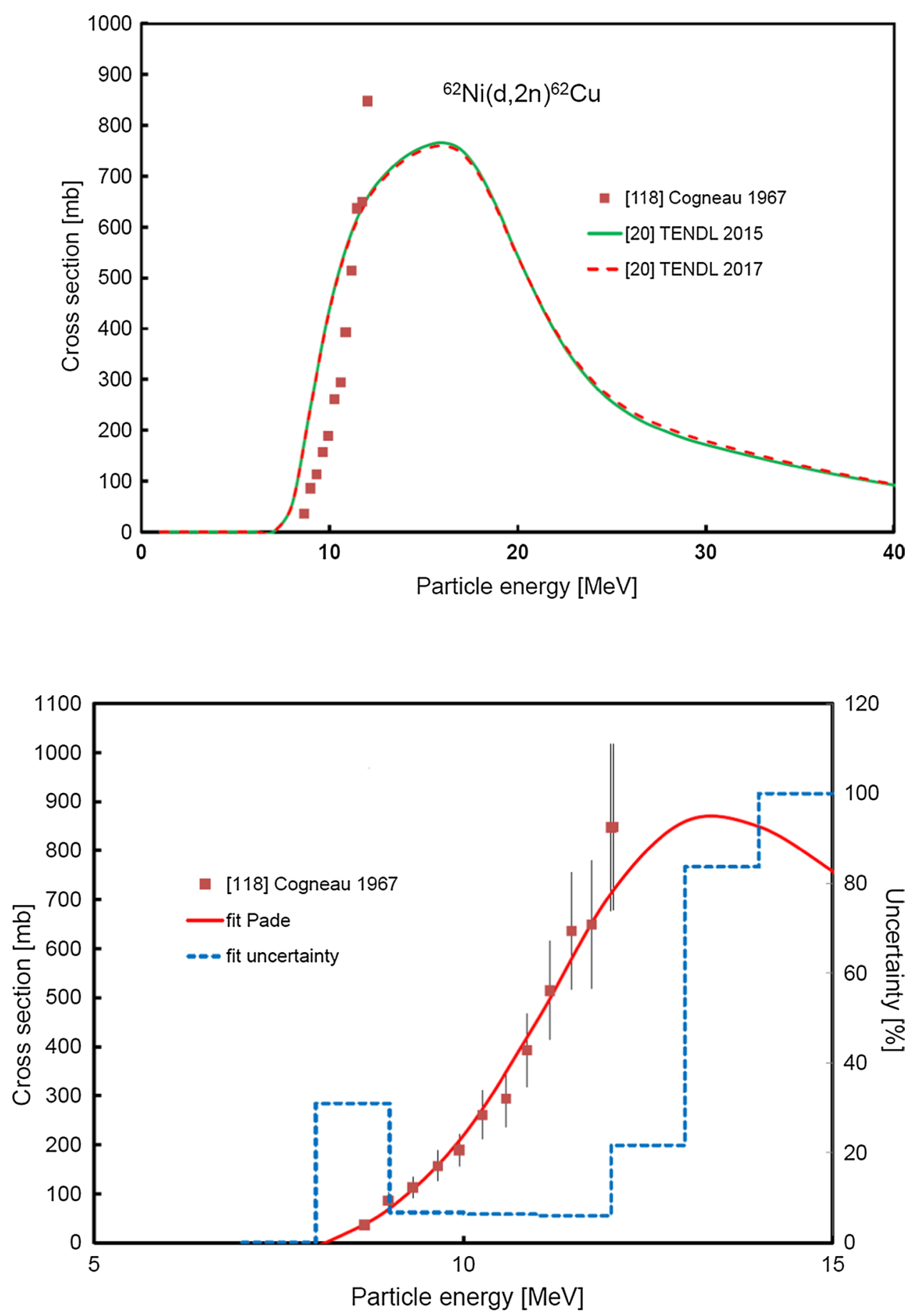
Thick target yields for production of ${ }^{62} \mathrm{Cu}$, and ${ }^{62} \mathrm{Zn}$ parent

See Figs. 54 and 55.

Fig. 54 Thick target yields calculated from the recommended cross sections for the ${ }^{62} \mathrm{Ni}(\mathrm{p}, \mathrm{n})^{62} \mathrm{Cu}$ and ${ }^{62} \mathrm{Ni}(\mathrm{d}, 2 \mathrm{n}){ }^{62} \mathrm{Cu}$ reactions

Fig. 55 Thick target yields calculated from the recommended cross sections for the ${ }^{63} \mathrm{Cu}(\mathrm{p}, 2 \mathrm{n}){ }^{62} \mathrm{Zn},{ }^{63} \mathrm{Cu}(\mathrm{d}, 3 \mathrm{n}){ }^{62} \mathrm{Zn}$ and ${ }^{\text {nat }} \mathrm{Ni}(\boldsymbol{\alpha}, \mathrm{xn})^{62} \mathrm{Zn}$ reactions
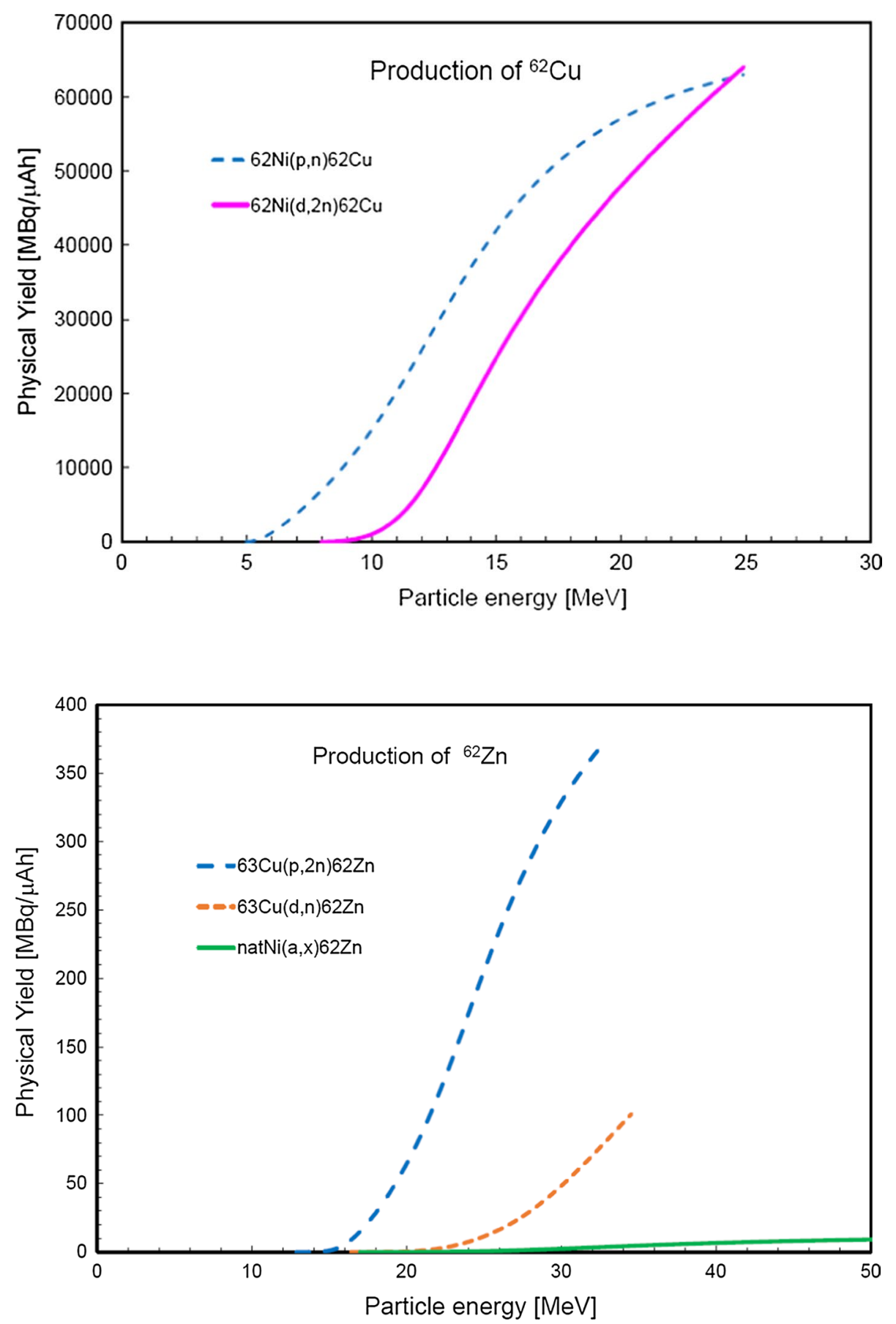


\section{Production of ${ }^{66} \mathrm{Ga}\left(T_{1 / 2}=9.49 \mathrm{~h}\right)$}

Applications: Both ${ }^{66} \mathrm{Ga}$ and ${ }^{68} \mathrm{Ga}$ are positron-emitting radionuclides that can be used in PET imaging. Longerlived ${ }^{66} \mathrm{Ga}$ has been coupled to monoclonal antibodies (e.g., for tumour angiogenesis studies) and to nanoparticles. This radionuclide has also been proposed in hadron therapy as an in situ marker for the incorporation of $\mathrm{Zn}$ in tumours. Obvious disadvantages are the rather high radiation burden and inferior imaging properties caused by the many gamma rays that accompany decay.

${ }^{66} \mathrm{Ga}(9.49 h): \beta^{+}(57 \%)$, and $E_{\gamma}(\mathrm{keV})\left(P_{\gamma}(\%)\right): 833.5324$ (5.9), 1039.220 (37.0).

Evaluations have been made of the ${ }^{66} \mathrm{Zn}(\mathrm{p}, \mathrm{n}){ }^{66} \mathrm{Ga}$ and ${ }^{63} \mathrm{Cu}(\alpha, \mathrm{n}){ }^{66} \mathrm{Ga}$ direct production routes.

\section{${ }^{66} \mathrm{Zn}(\mathrm{p}, \mathrm{n}){ }^{66} \mathrm{Ga}$}

The twenty experimental datasets available in the literature are shown in Fig. 56 [36, 56, 59, 124, 125, 164-177] together with the TENDL-2015 and TENDL-2017 calculations. Hermanne [173] contains two datasets labelled (a) and (b).Twelve datasets were rejected (Little and Lagunas-Solar [167] (values too low), Nortier et al. [171] (energy shift), Blosser and Handley [56] (only one data point that can not be checked), Howe [165] (energy shift), Kopecký [168] (values too low), Asad et al. [125] (values too low), Szelecsényi et al. [172] (preliminary results), Hermanne [173] set $\mathrm{b}$ (discrepant data points), Barrandon et al. [59] (values too low), Al-Saleh et al. [177] (values too low), Uddin et al. [124] (values too low), and Blaser et al. [164] (discrepant data points)), while the remaining eight sets were used in the statistical fitting procedure. The selected data and their experimental uncertainties are shown in Fig. 57 together with the Padé ( $\left.L=13, N=188, \chi^{2}=1.87\right)$ and estimated uncertainty in percentages, including $4 \%$ systematic uncertainty (right-hand scale).
Fig. 56 Twenty experimental datasets) for the ${ }^{66} \mathrm{Zn}(\mathrm{p}, \mathrm{n})^{66} \mathrm{Ga}$ reaction available in the literature $[36,56,59,124,125$, 164-177], and TENDL calculations. Ref. [173] contains two datasets labelled (a) and (b)

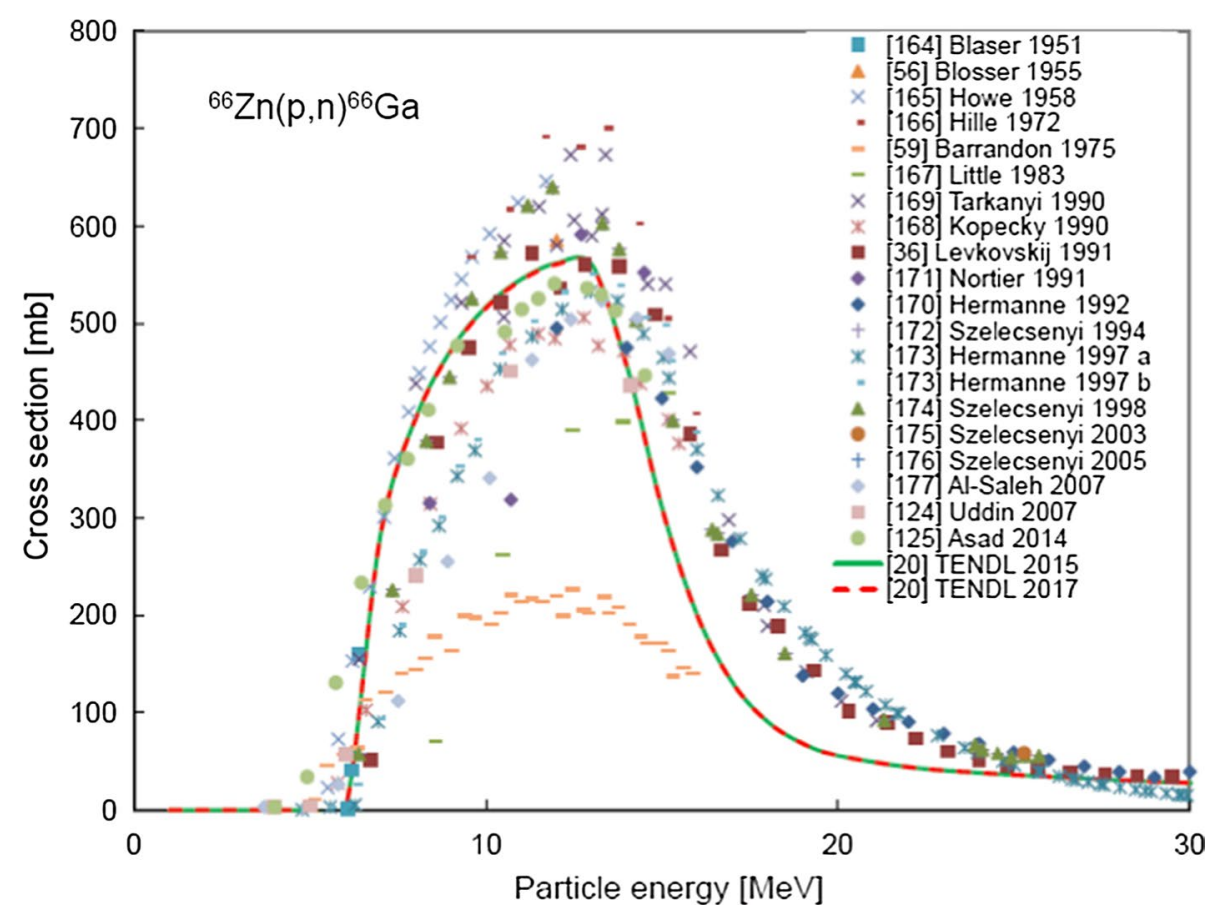


Fig. 57 Eight selected experimental datasets for the ${ }^{66} \mathrm{Zn}(\mathrm{p}, \mathrm{n}){ }^{66} \mathrm{Ga}$ reaction $[36$, $166,169,170,173-176]$ with the Padé fit ( $L=13, N=188$, $\chi^{2}=1.87$, solid line) and estimated total uncertainties in percentages, including $4 \%$ systematic uncertainty (dashed line, right-hand scale)

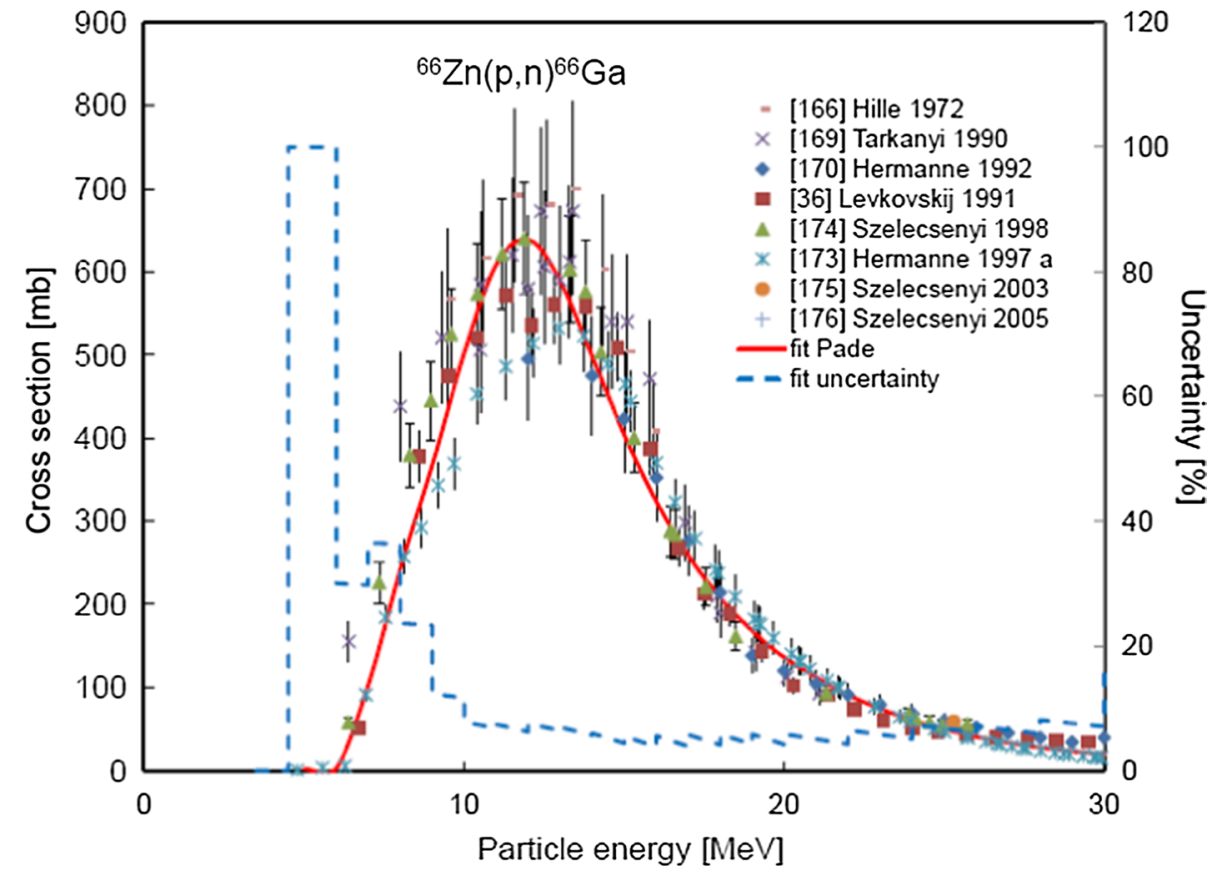




\section{${ }^{63} \mathrm{Cu}(\mathrm{a}, \mathrm{n}){ }^{66} \mathrm{Ga}$}

The twenty-three experimental datasets available in the literature are shown in Fig. 58 [36, 54, 81, 166, 178-196] together with the TENDL calculations. Seven datasets were rejected (Porges [178] (values too low), Bonesso et al. [188] (values too low), Zhukova et al. [182] (values too low), Singh et al. [190] (values too low), Rizvi et al. [184] (values too low), Porile and Morrison [179] (values too low), and Nassiff and Nassiff [183] (discrepant data points)), and the remaining sixteen sets were used in the statistical fitting procedure. The selected data and their experimental uncertainties are shown in Fig. 59 together with the Padé $(L=13$, $\left.N=252, \chi^{2}=1.34\right)$ and estimated uncertainty in percentages, including $4 \%$ systematic uncertainty (right-hand scale). This reaction is also used to monitor $\alpha$-particle beams (see Ref. [30], Section V D).
Fig. 58 Twenty-three experimental datasets for the ${ }^{63} \mathrm{Cu}(\alpha, \mathrm{n}){ }^{66} \mathrm{Ga}$ reaction available in the literature $[36,54,81$, 166, 178-196], and TENDL calculations

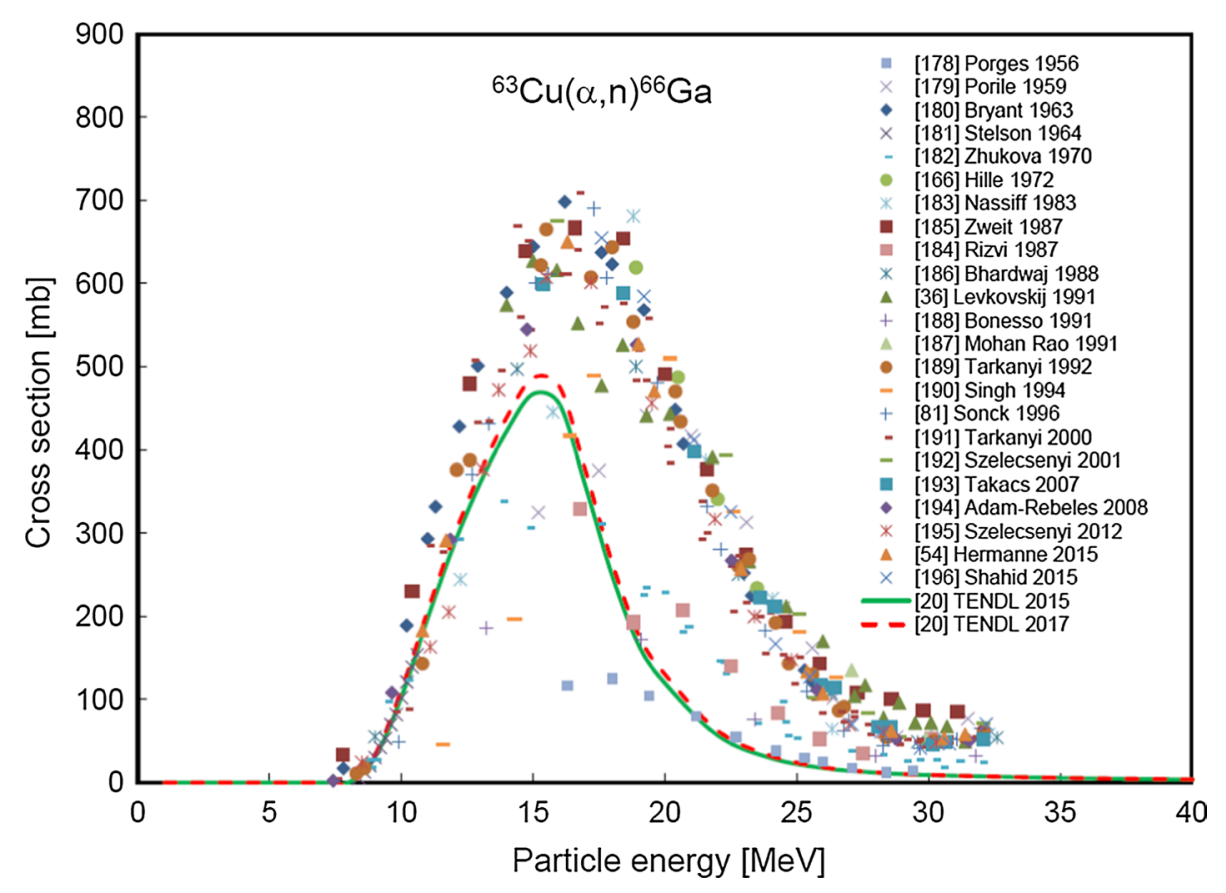

Fig. 59 Sixteen selected experimental datasets for the ${ }^{63} \mathrm{Cu}(\alpha, \mathrm{n}){ }^{66} \mathrm{Ga}$ reaction $[36,54$, $81,166,180,181,185-187$, $189,191-196]$ with the Padé fit $\left(L=13, N=252, \chi^{2}=1.34\right.$, solid line) and estimated total uncertainties in percentages, including $4 \%$ systematic uncertainty (dashed line, right-hand scale)

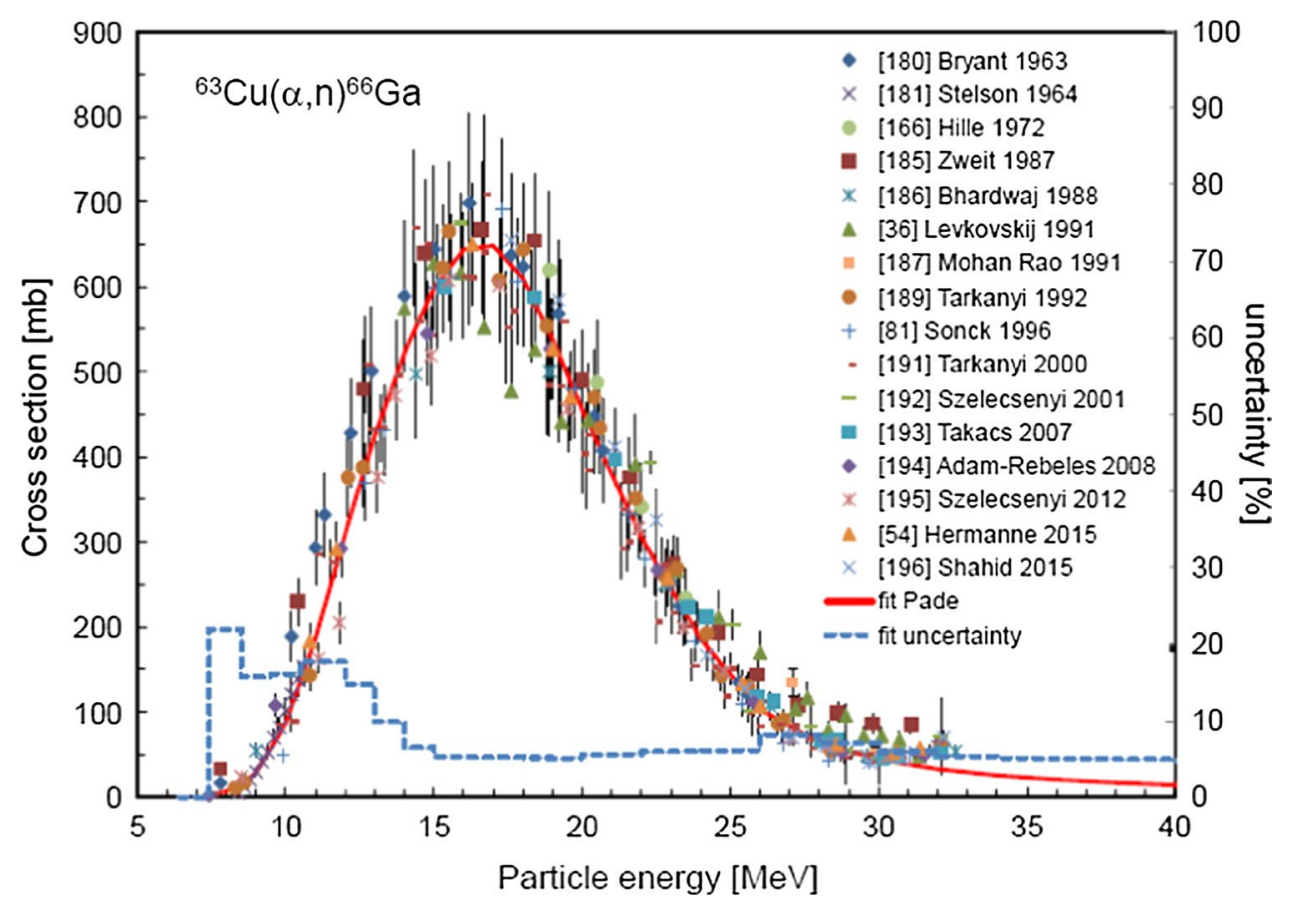


Thick target yields for production of ${ }^{66} \mathrm{Ga}$

See Fig. 60.

Fig. 60 Thick target yields calculated from the recommended cross sections for the ${ }^{66} \mathrm{Zn}(\mathrm{p}, \mathrm{n}){ }^{66} \mathrm{Ga}$ and

${ }^{63} \mathrm{Cu}(\alpha, \mathrm{n}){ }^{66} \mathrm{Ga}$ reactions

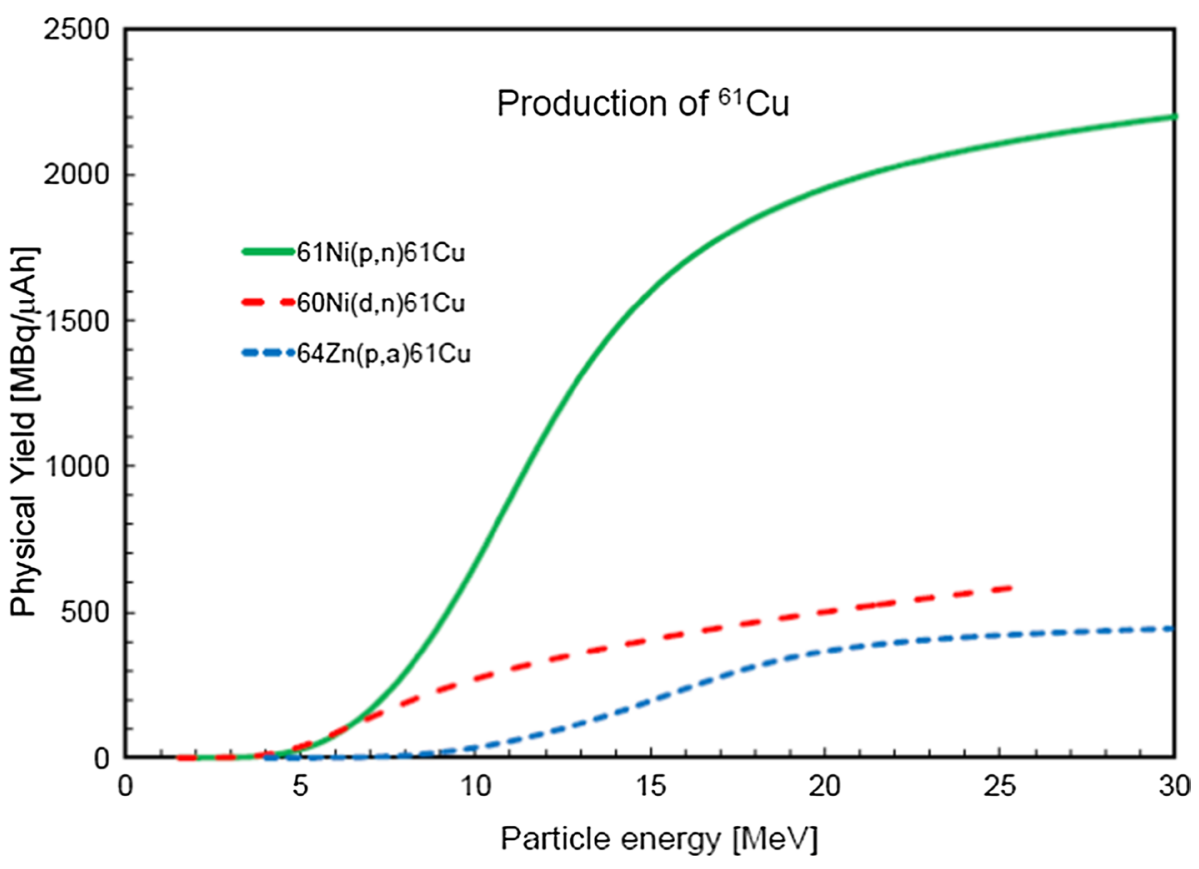




\section{Production of ${ }^{68} \mathrm{Ga}\left(T_{1 / 2}=67.71 \mathrm{~min}\right)$ and long-lived ${ }^{68} \mathrm{Ge}$ parent $\left(T_{1 / 2}=270.95 \mathrm{~d}\right)$}

Applications: Rather short-lived ${ }^{68} \mathrm{Ga}$ became the first widespread generator-produced positron emitter, thereby competing somewhat with ${ }^{18} \mathrm{~F}$ for preferred adoption in PET imaging. First introduced for the imaging of neuroendocrine tumours ( ${ }^{68} \mathrm{Ga}$-labelled DOTA-TOC), more recent significant success has been achieved in the form of very efficient imaging agents for prostate cancer diagnosis and staging ( ${ }^{68} \mathrm{Ga}$-DOTA-PSMA and derivatives).

${ }^{68} \mathrm{Ga}(67.71 \mathrm{~min}): \beta^{+}(88.91 \%)$, and $E_{\gamma}(\mathrm{keV})\left(P_{\gamma}(\%)\right)$ : 1077.34 (3.22).

${ }^{68} \mathrm{Ge}(270.95 \mathrm{~d})$ : detected by means of radiation from daughter ${ }^{68} \mathrm{Ga}$.

Evaluations have been undertaken of the ${ }^{68} \mathrm{Zn}(\mathrm{p}, \mathrm{n}){ }^{68} \mathrm{Ga}$ and ${ }^{65} \mathrm{Cu}(\alpha, \mathrm{n}){ }^{68} \mathrm{Ga}$ direct routes and ${ }^{\text {nat }} \mathrm{Ga}(\mathrm{p}, \mathrm{x}){ }^{68} \mathrm{Ge}$ and ${ }^{69} \mathrm{Ga}(\mathrm{p}, 2 \mathrm{n}){ }^{68} \mathrm{Ge}$ generator production.
${ }^{68} \mathrm{Zn}(\mathrm{p}, \mathrm{n}){ }^{68} \mathrm{Ga}$

The eighteen experimental datasets available in the literature are shown in Fig. 61 [36, 41, 56, 59, 111, 162, 164-166, 169, $170,173,177,195,197-200]$ together with the TENDL calculations. Five datasets were rejected (Hermanne et al. [170] (energy shift), Blosser and Handley [56] (value too high), McGee et al. [41] (value too low), Hermanne [173] (energy shift), and Barrandon et al. [59] (values too low)), and the remaining thirteen sets were used in the statistical fitting procedure. The selected data and their experimental uncertainties are shown in Fig. 62 together with the Padé $(L=20$, $\left.N=282, \chi^{2}=1.97\right)$ and estimated uncertainty in percentages, including $4 \%$ systematic uncertainty (right-hand scale).
Fig. 61 Eighteen experimental datasets for the ${ }^{68} \mathrm{Zn}(\mathrm{p}, \mathrm{n})^{68} \mathrm{Ga}$ reaction available in the literature $[36,41,56,59,111,162$, 164-166, 169, 170, 173, 177, 195, 197-200], and TENDL calculations

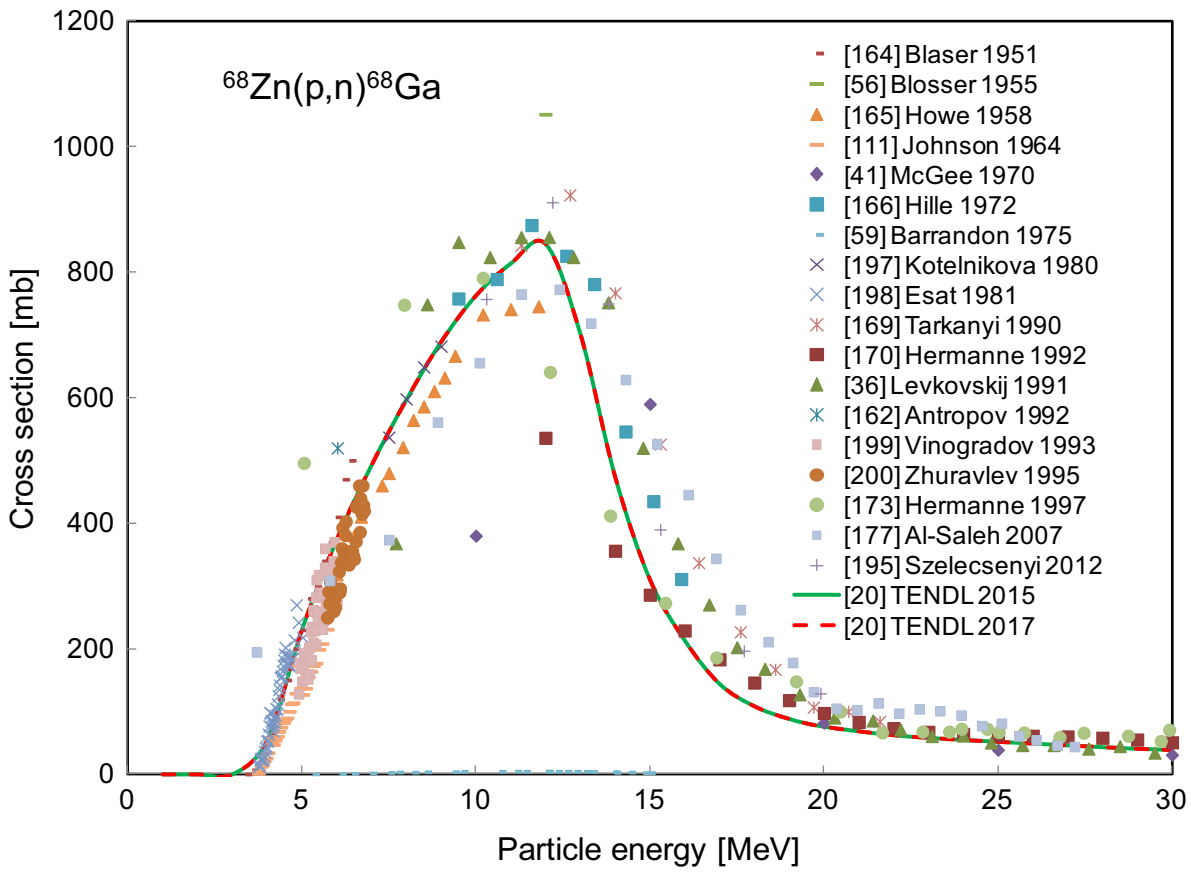


Fig. 62 Thirteen selected experimental datasets for the ${ }^{68} \mathrm{Zn}(\mathrm{p}, \mathrm{n}){ }^{68} \mathrm{Ga}$ reaction $[36$, 111, 162, 164-166, 169, 177, 195, 197-200] with the Padé fit $\left(L=20, N=282, \chi^{2}=1.97\right.$, solid line) and estimated total uncertainties in percentages, including 4\% systematic uncertainty (dashed line, right-hand scale)

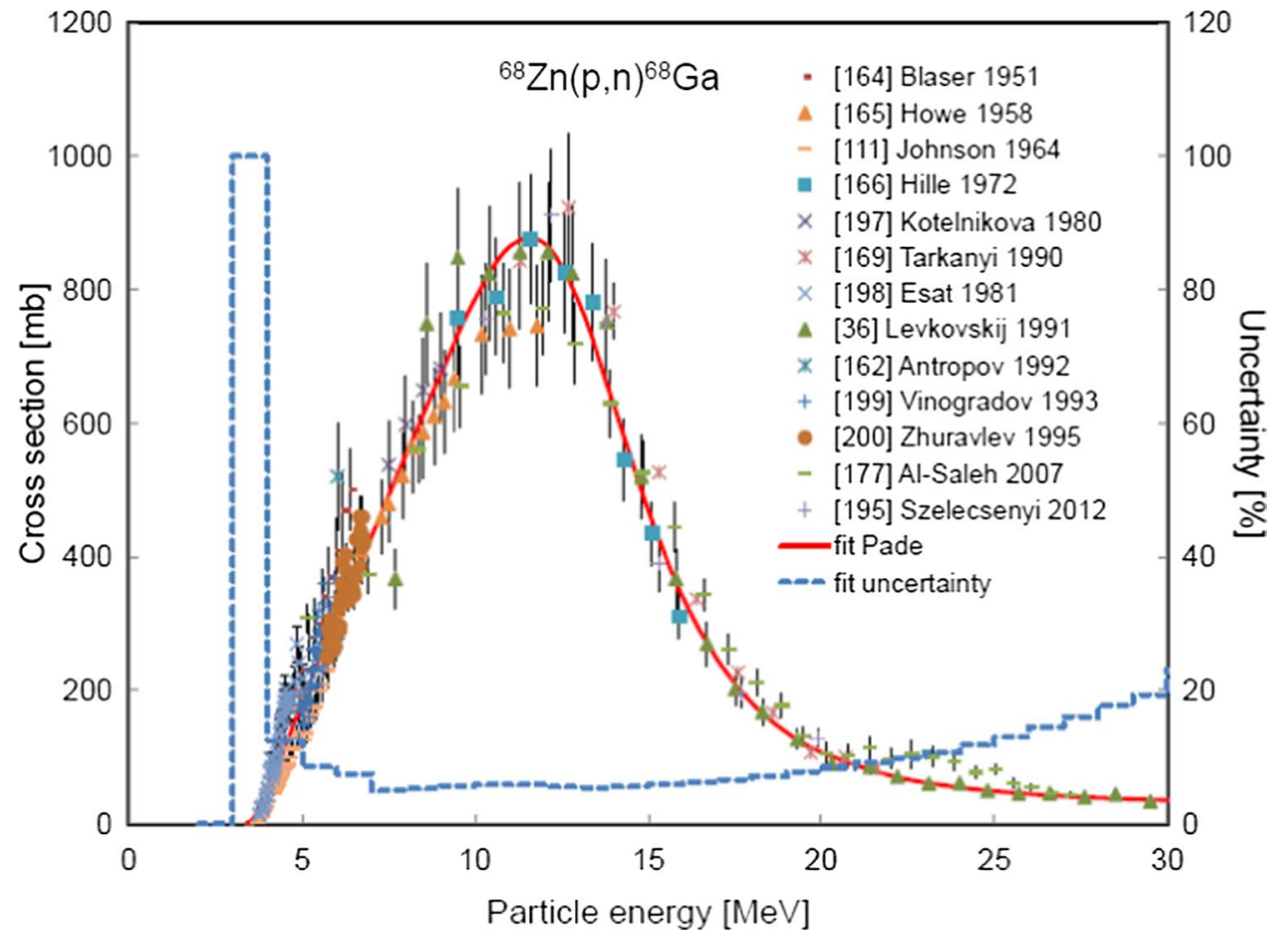




\section{${ }^{65} \mathrm{Cu}(a, n){ }^{68} \mathrm{Ga}$}

The fourteen experimental datasets available in the literature are shown in Fig. 63 [36, 54, 166, 178-180, 184, 186, 188, $190,195,196,201,202]$ together with the TENDL calculations. Four datasets were rejected (Porile and Morrison [179] (energy shift), Rizvi et al. [184] (energy shift), Bonesso et al.
[188] (values too high), and Porges [178] (values too low)), and the remaining ten sets were used in the statistical fitting procedure. The selected data and their experimental uncertainties are shown in Fig. 64 together with the Padé $(L=10$, $N=92, \chi^{2}=1.21$ ) and estimated uncertainty in percentages, including $4 \%$ systematic uncertainty (right-hand scale).
Fig. 63 Fourteen experimental datasets for the ${ }^{65} \mathrm{Cu}(\alpha, n){ }^{68} \mathrm{Ga}$ reaction available in the literature $[36,54,166,178-180,184$, $186,188,190,195,196,201$, 202], and TENDL calculations

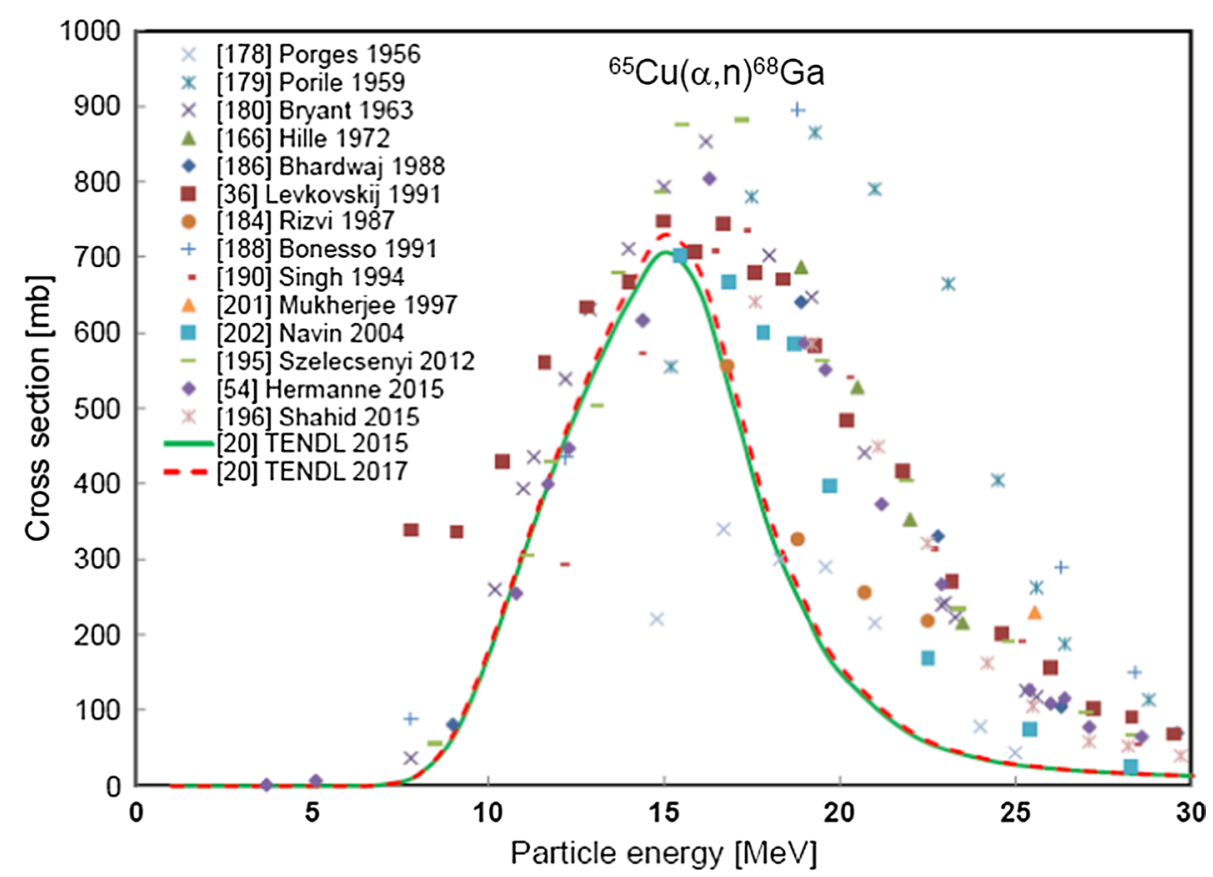

Fig. 64 Ten selected experimental datasets for the ${ }^{65} \mathrm{Cu}(\alpha, \mathrm{n}){ }^{68} \mathrm{Ga}$ reaction [36, 54, 166, 180, 186, 190, 195, $196,201,202]$ with the Padé fit $\left(L=10, N=92, \chi^{2}=1.21\right.$, solid line) and estimated total uncertainties in percentages, including $4 \%$ systematic uncertainty (dashed line, right-hand scale)

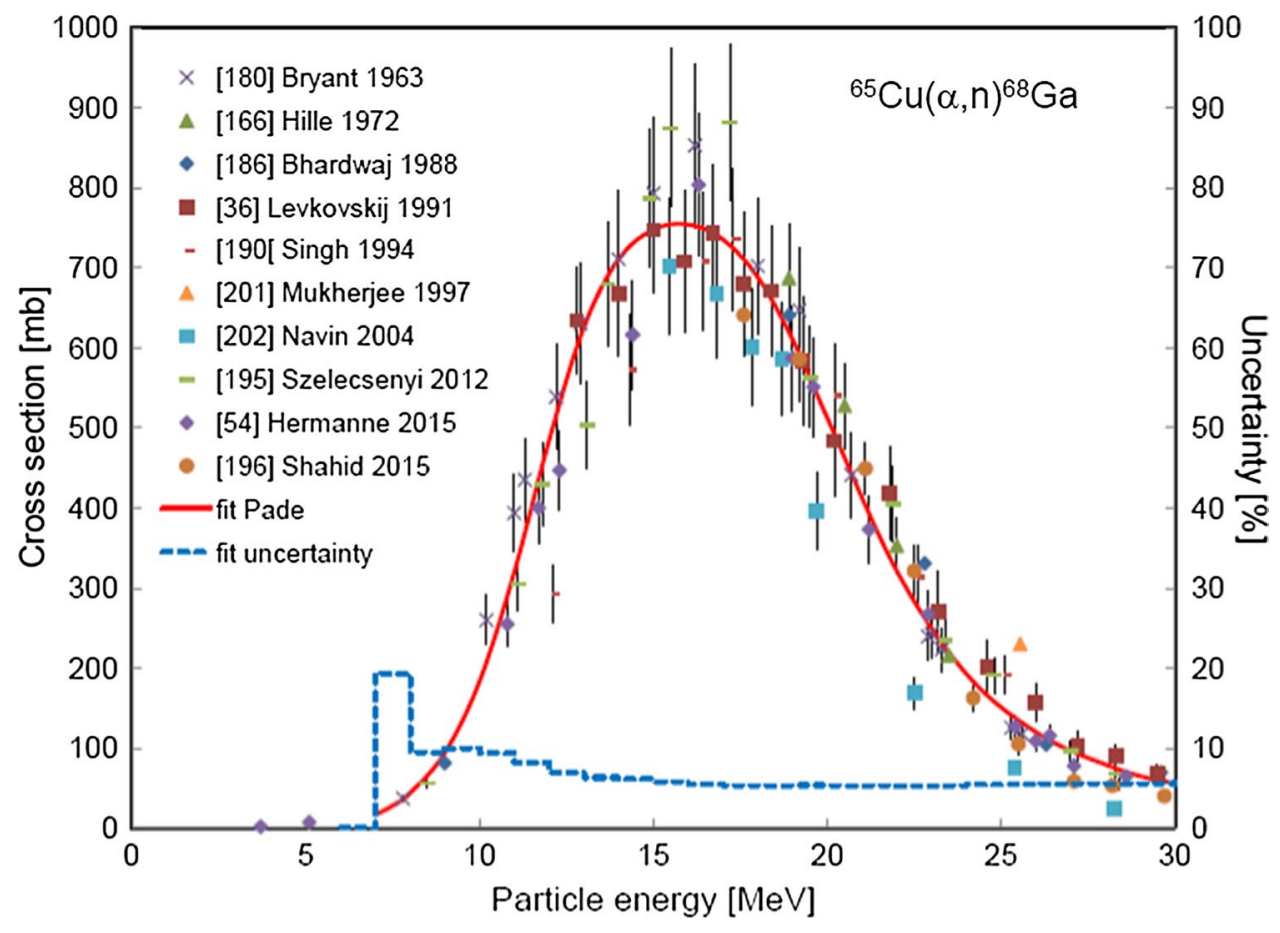


${ }^{\text {nat }} \mathrm{Ga}(\mathrm{p}, \mathrm{xn}){ }^{68} \mathrm{Ge}$

The six experimental datasets available in the literature are shown in Fig. 65 [36, 48, 98, 203, 204] together with the TENDL calculations. Hermanne et al. [48] contains two datasets labelled (a) and (b). One dataset was rejected
(Cohen and Newman [98], single data point too low), and the remaining five sets were used in the statistical fitting procedure. The selected data and their experimental uncertainties are shown in Fig. 66 together with the Padé $(L=11$, $N=101, \chi^{2}=1.42$ ) and estimated uncertainty in percentages, including $4 \%$ systematic uncertainty (right-hand scale).
Fig. 65 Six experimental datasets for the ${ }^{\text {nat }} \mathrm{Ga}(\mathrm{p}, \mathrm{xn}){ }^{68} \mathrm{Ge}$ reaction available in the literature [36, 48, 98, 203, 204], and TENDL calculations. Hermanne et al. [48] contains two sets of data labelled (a) and (b)

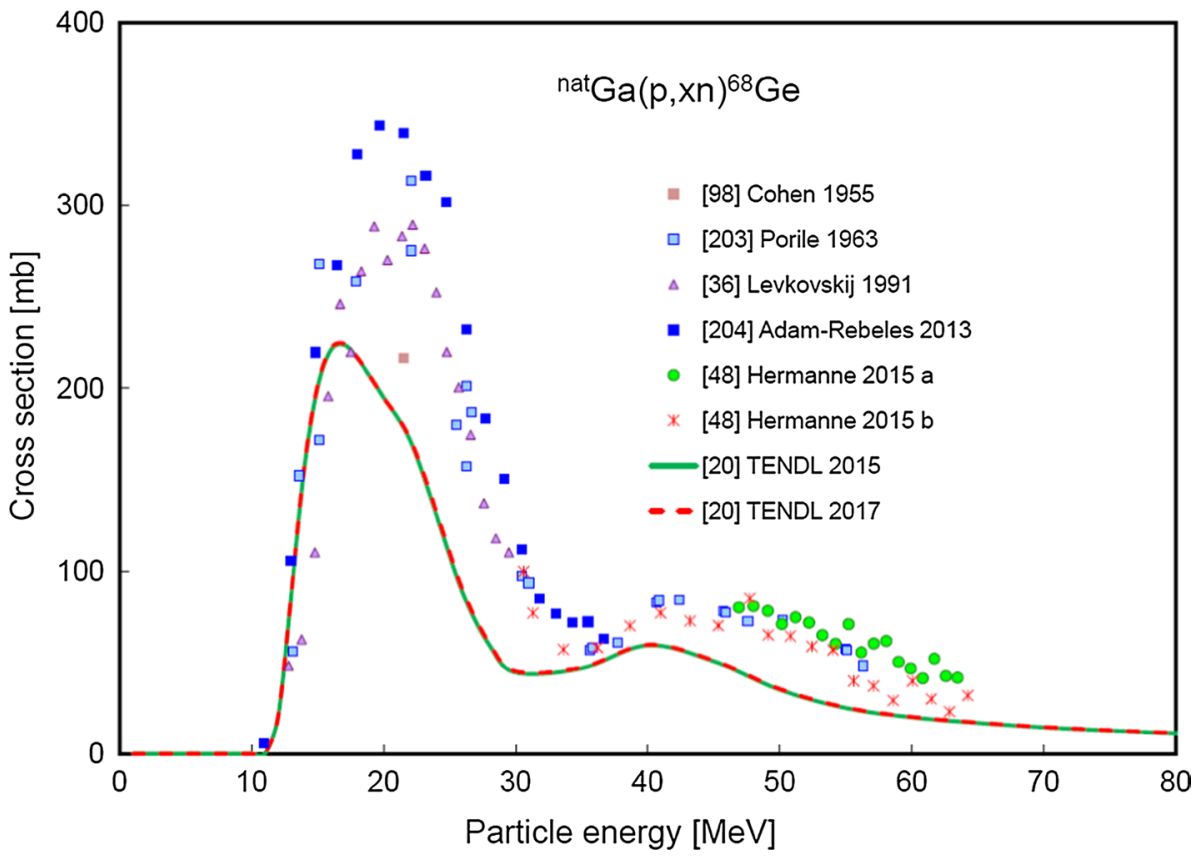

Fig. 66 Five selected experimental datasets for the ${ }^{\text {nat }} \mathrm{Ga}(\mathrm{p}, \mathrm{xn}){ }^{68} \mathrm{Ge}$ reaction [36, $48,203,204]$ with the Padé fit $\left(L=11, N=101, \chi^{2}=1.42\right.$, solid line) and estimated total uncertainties in percentages, including $4 \%$ systematic uncertainty (dashed line, right-hand scale)

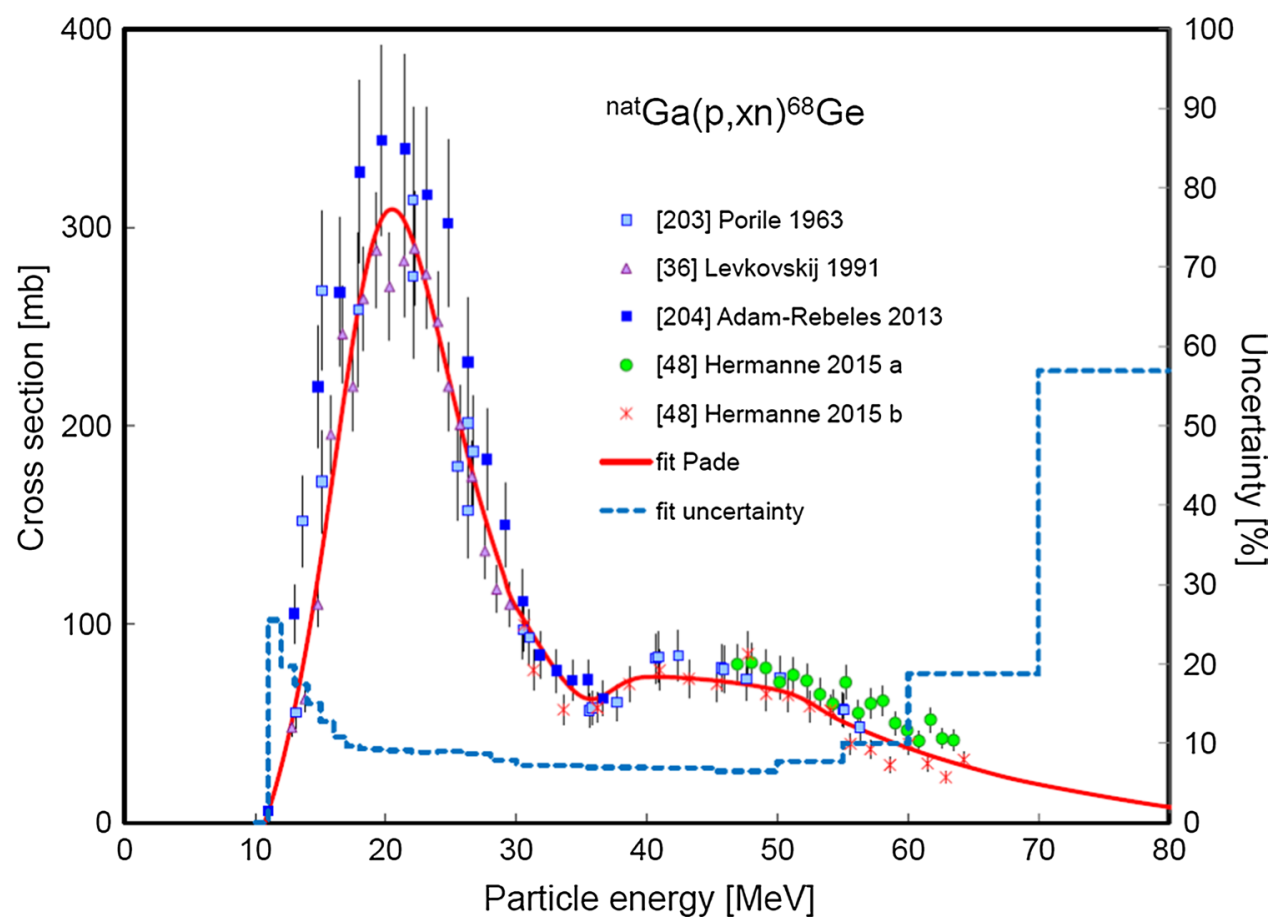




\section{${ }^{69} \mathrm{Ga}(\mathrm{p}, 2 \mathrm{n}){ }^{68} \mathrm{Ge}$}

The four experimental datasets available in the literature are shown in Fig. 67 [36, 48, 203, 204] together with the TENDL calculations. All sets were used in the statistical fitting procedure. The data and their experimental uncertainties are shown in Fig. 68 together with the Padé $(L=8$, $N=53, \chi^{2}=1.56$ ) and estimated uncertainty in percentages, including $4 \%$ systematic uncertainty (right-hand scale).
Fig. 67 Four experimental datasets for the ${ }^{69} \mathrm{Ga}(\mathrm{p}, 2 \mathrm{n})^{68} \mathrm{Ge}$ reaction available in the literature [36, 48, 203, 204], and TENDL calculations
Fig. 68 Four experimental datasets for the ${ }^{69} \mathrm{Ga}(\mathrm{p}, 2 \mathrm{n}){ }^{68} \mathrm{Ge}$ reaction [36, 48, 203, 204] with the Padé fit $(L=8, N=53$, $\chi^{2}=1.56$, solid line) and estimated total uncertainties in percentages, including $4 \%$ systematic uncertainty (dashed line, right-hand scale)
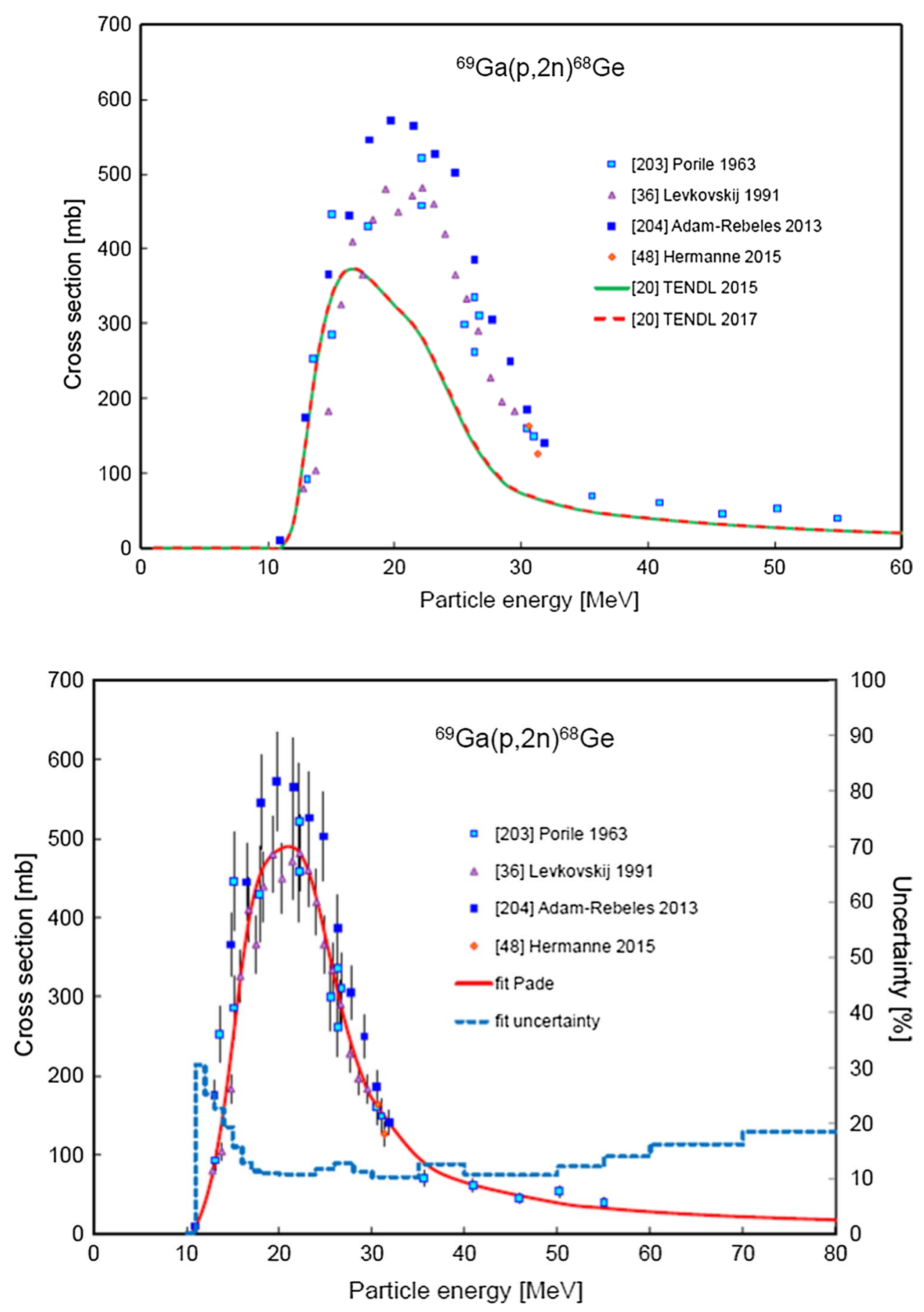
Thick target yields for ${ }^{68} \mathrm{Ga}$, and long-lived ${ }^{68} \mathrm{Ge}$ parent for generator

See Figs. 69 and 70.

Fig. 69 Thick target yields calculated from the recommended cross sections for the ${ }^{68} \mathrm{Zn}(\mathrm{p}, \mathrm{n})^{68} \mathrm{Ga}$ and ${ }^{65} \mathrm{Cu}(\alpha, \mathrm{n}){ }^{68} \mathrm{Ga}$ direct reactions

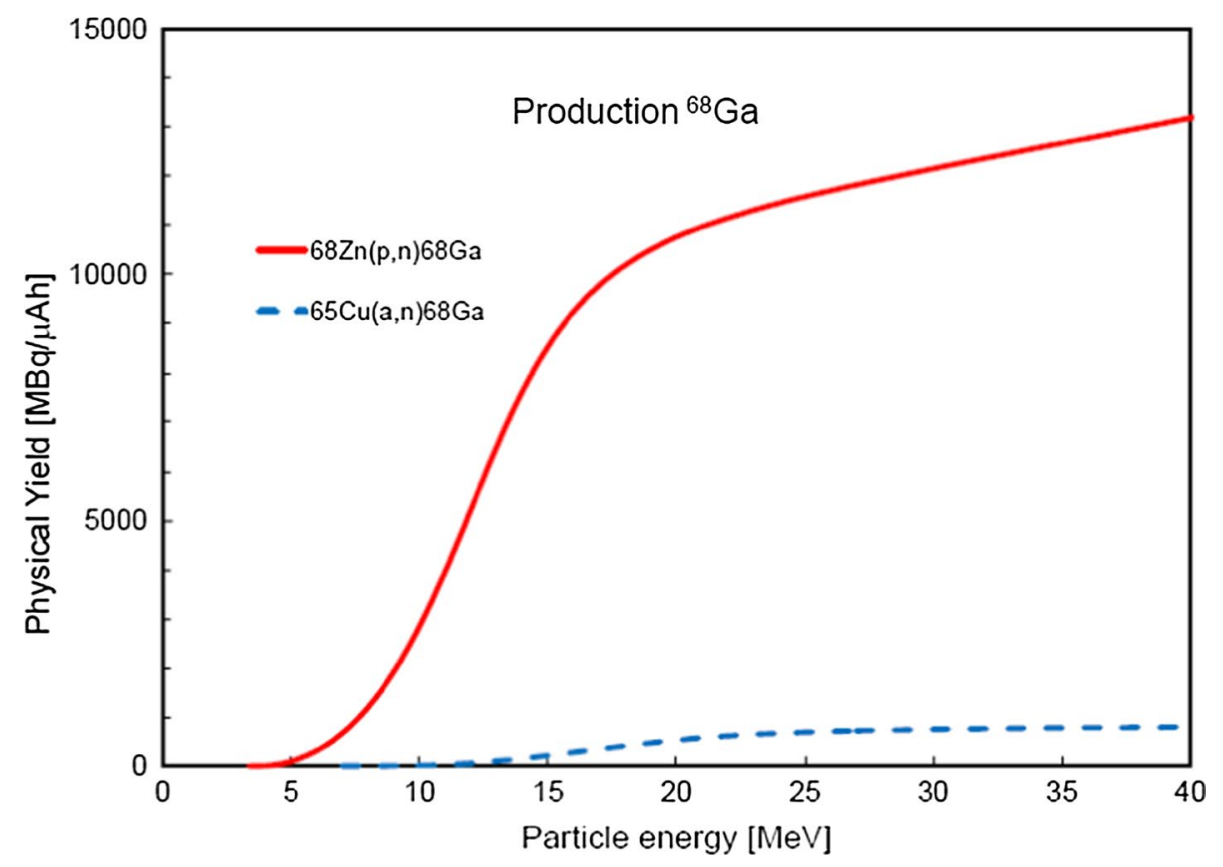

Fig. 70 Thick target yields calculated from the recommended cross sections for the ${ }^{\text {nat }} \mathrm{Ga}(\mathrm{p}, \mathrm{xn}){ }^{68} \mathrm{Ge}$ and ${ }^{69} \mathrm{Ga}(\mathrm{p}, 2 \mathrm{n}){ }^{68} \mathrm{Ge}$ reactions to produce long-lived parent for ${ }^{68} \mathrm{Ga}$ generator

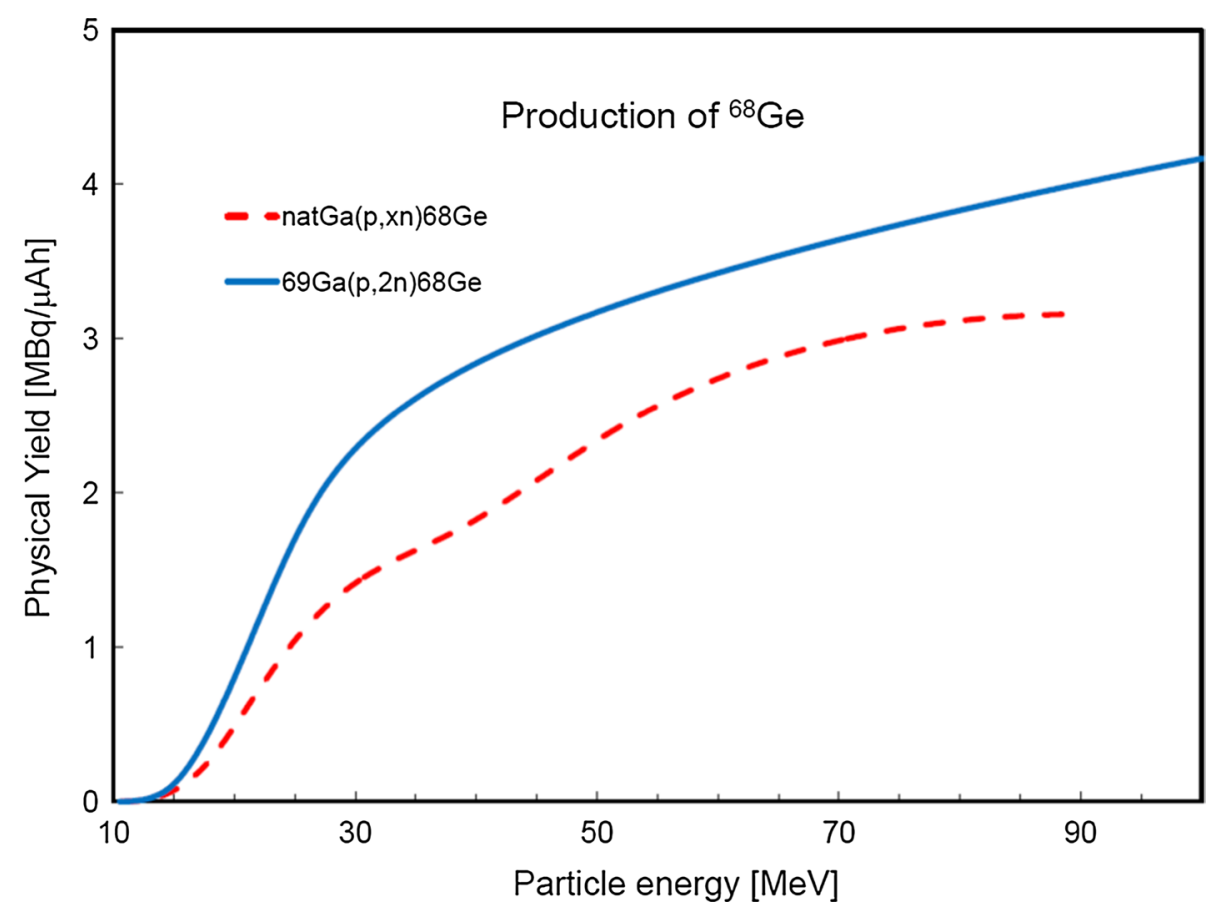




\section{Production of ${ }^{72} \mathrm{As}\left(T_{1 / 2}=26.0 \mathrm{~h}\right)$ and longer-lived ${ }^{72}$ Se parent $\left(T_{1 / 2}=8.40 \mathrm{~d}\right)$}

Applications: ${ }^{72} \mathrm{As}$ is a long-lived positron-emitting radionuclide suitable for imaging the bio-distribution of monoclonal antibodies with long biological half-lives that are promising in PET oncological research. Chemical properties offer the possibility of covalent bonding to thiol groups.

${ }^{72} A s(26.0 \mathrm{~h}): \beta^{+}(87.8 \%)$, and $E_{\gamma}(\mathrm{keV})\left(P_{\gamma}(\%)\right): 629.92$ (8.07), 833.99 (81).

${ }^{72} \mathrm{Se}(8.40 \mathrm{~d})$ : detected by means of radiation emitted by daughter ${ }^{72}$ As.

Evaluations have been undertaken of the ${ }^{75} \mathrm{As}(\mathrm{p}, 4 \mathrm{n}){ }^{72} \mathrm{Se}$ and ${ }^{n a t} \operatorname{Br}(p, x){ }^{72} \mathrm{Se}$ routes for parent production, and the
${ }^{\text {nat }} \mathrm{Ge}(\mathrm{p}, \mathrm{xn}){ }^{72} \mathrm{As}$ and ${ }^{\text {nat }} \mathrm{Ge}(\mathrm{d}, \mathrm{xn}){ }^{72} \mathrm{As}$ direct production routes.

\section{${ }^{75} \mathrm{As}(p, 4 n){ }^{72} \mathrm{Se}$}

The two experimental datasets available in the literature for the energy domain considered are shown in Fig. 71 [205, 206] together with the TENDL calculations. Both datasets were used in the statistical fitting procedure. The data and their experimental uncertainties are shown in Fig. 72 together with the Padé fit $\left(L=8, N=33, \chi^{2}=1.30\right)$ and estimated uncertainty in percentages, including $4 \%$ systematic uncertainty (right-hand scale).
Fig. 71 Two experimental datasets for the ${ }^{75} \mathrm{As}(\mathrm{p}, 4 \mathrm{n})^{72} \mathrm{Se}$ reaction available in the literature [205, 206], and TENDL calculations

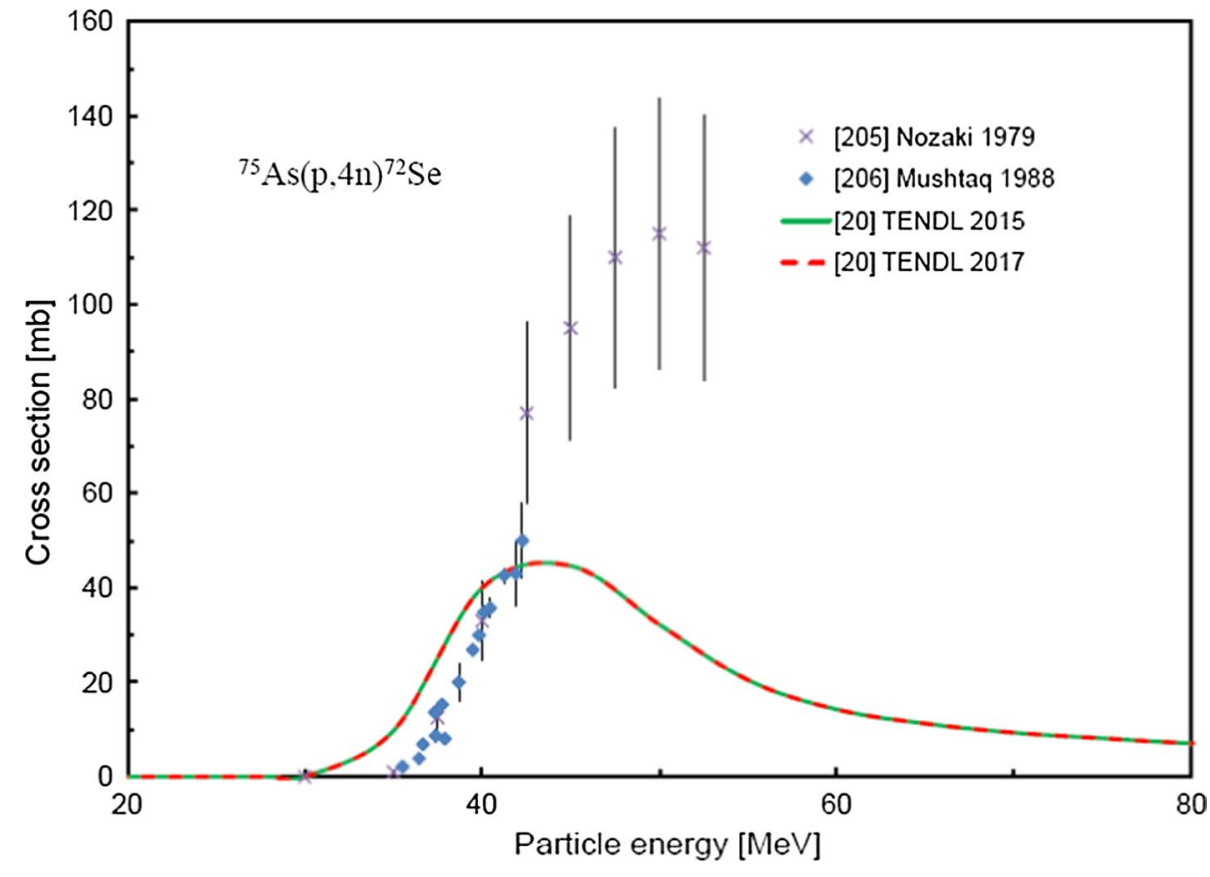


Fig. 72 Two experimental datasets for the ${ }^{75} \mathrm{As}(\mathrm{p}, 4 \mathrm{n}){ }^{72} \mathrm{Se}$ reaction $[205,206]$ with the Padé fit $\left(L=8, N=33, \chi^{2}=1.30\right.$, solid line) and estimated total uncertainties in percentages, including $4 \%$ systematic uncertainty (dashed line, right-hand scale)

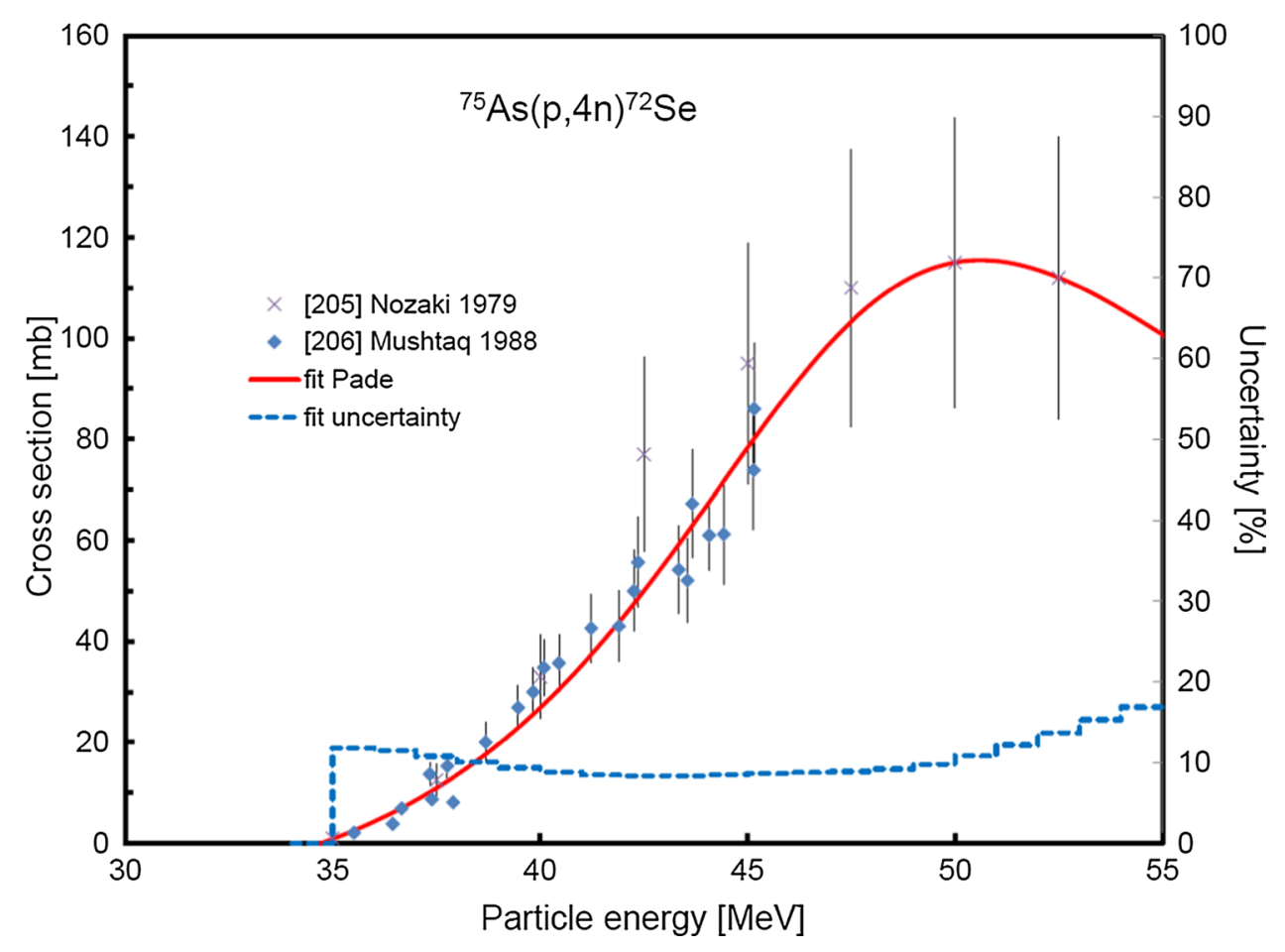




\section{${ }^{\text {nat }} \mathrm{Br}(\mathrm{p}, \mathrm{x})^{72} \mathrm{Se}$}

The two experimental datasets available in the literature are shown in Fig. 73 [207, 208] together with the TENDL calculations. Both sets of measurements by Fassbender et al. [207] and de Villiers et al. [208] originate from the same experimental study, and should be identical. Therefore, the data of de Villers et al. [208] were set aside, while only the other dataset was used in the statistical fitting procedure. These selected data and their experimental uncertainties are shown in Fig. 74 together with the Padé fit $(L=10, N=14$, $\chi^{2}=0.35$ ) and estimated uncertainty in percentages, including $4 \%$ systematic uncertainty (right-hand scale). The contributions of the similar (p,2pxn) reactions on the two stable isotopes of $\mathrm{Br}$ can be clearly distinguished $\left({ }^{79} \mathrm{Br}\right.$ : $50.69 \%$; $\left.{ }^{81} \mathrm{Br}: 49.31 \%\right)$.
Fig. 73 Two experimental datasets for the ${ }^{\text {nat }} \mathrm{Br}(\mathrm{p}, \mathrm{x})^{72} \mathrm{Se}$ reaction available in the literature [207, 208], and TENDL calculations
Fig. 74 One selected experimental dataset for the ${ }^{n a t} \operatorname{Br}(\mathrm{p}, \mathrm{x})^{72} \mathrm{Se}$ reaction [207] with the Padé fit $(L=10, N=14$, $\chi^{2}=0.35$, solid line) and estimated total uncertainties in percentages, including $4 \%$ systematic uncertainty (dashed line, right-hand scale)
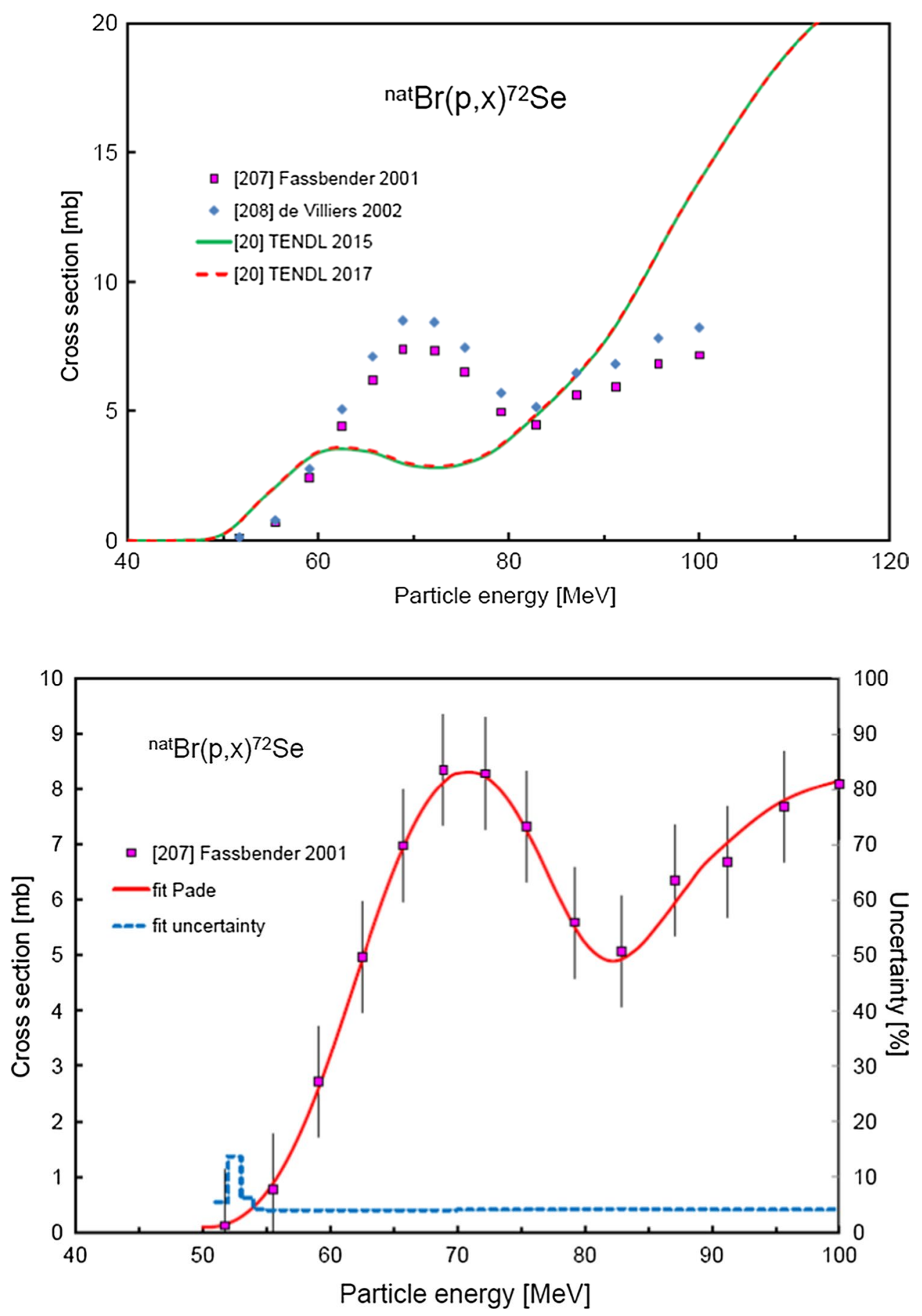


\section{${ }^{\text {nat }} \mathrm{Ge}(\mathrm{p}, \mathrm{xn}){ }^{72} \mathrm{As}$}

The four experimental datasets available in the literature are shown in Fig. 75 [36, 209-211] together with the TENDL calculations. All datasets were used in the statistical fitting procedure. The data and their experimental uncertainties are shown in Fig. 76 together with the Padé $(L=18, N=123$, $\left.\chi^{2}=1.97\right)$ and estimated uncertainty in percentages, including $4 \%$ systematic uncertainty (right-hand scale). Contributions of similar $(\mathrm{p}, \mathrm{xn})$ reactions with increasing thresholds can be clearly distinguished for the higher abundance ${ }^{72} \mathrm{Ge},{ }^{74} \mathrm{Ge}$ and ${ }^{76} \mathrm{Ge}$.
Fig. 75 Four experimental datasets for the ${ }^{\text {nat }} \mathrm{Ge}(\mathrm{p}, \mathrm{xn})^{72} \mathrm{As}$ reaction available in the literature [36, 209-211], and TENDL calculations

Fig. 76 Four experimental datasets for the ${ }^{\text {nat }} \mathrm{Ge}(\mathrm{p}, \mathrm{xn})^{72} \mathrm{As}$ reaction [36, 209-211] with the Padé fit $(L=18, N=123$, $\chi^{2}=1.97$, solid line) and estimated total uncertainties in percentages, including $4 \%$ systematic uncertainty (dashed line, right-hand scale)
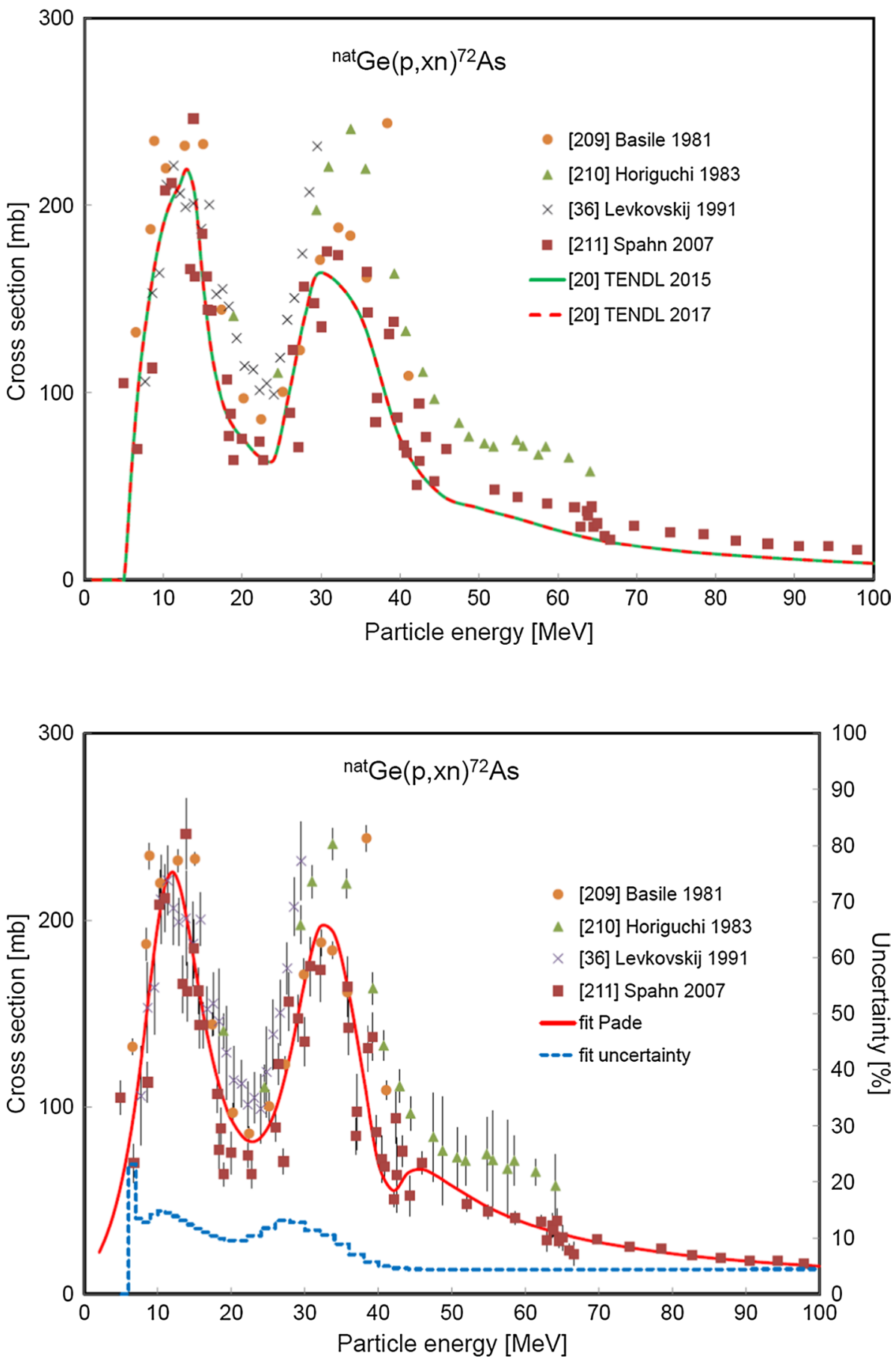


\section{${ }^{n a t} \mathrm{Ge}(\mathrm{d}, \mathrm{xn})^{72} \mathrm{As}$}

The single experimental dataset available in the literature is shown in Fig. 77 [212] together with the TENDL calculations. All data points and their experimental uncertainties are shown in Fig. 78 together with the Padé fit $(L=10$,
$N=25, \chi^{2}=1.13$ ) and estimated uncertainty in percentages, including $4 \%$ systematic uncertainty (right-hand scale). The contribitions of the ${ }^{72} \mathrm{Ge}(\mathrm{d}, 2 \mathrm{n})$ and ${ }^{74} \mathrm{Ge}(\mathrm{d}, 4 \mathrm{n})$ reactions on high natural abundance Ge isotopes can be seen in both figures.
Fig. 77 One experimental dataset for the ${ }^{\text {nat }} \mathrm{Ge}(\mathrm{d}, \mathrm{xn})^{72}$ As reaction available in the literature [212], and TENDL calculations
Fig. 78 One experimental dataset for the ${ }^{\text {nat }} \mathrm{Ge}(\mathrm{d}, \mathrm{xn})^{72} \mathrm{As}$ reaction [212] with the Padé fit $\left(L=10, N=25, \chi^{2}=1.13\right.$, solid line) and estimated total uncertainties in percentages, including $4 \%$ systematic uncertainty (dashed line, right-hand scale)
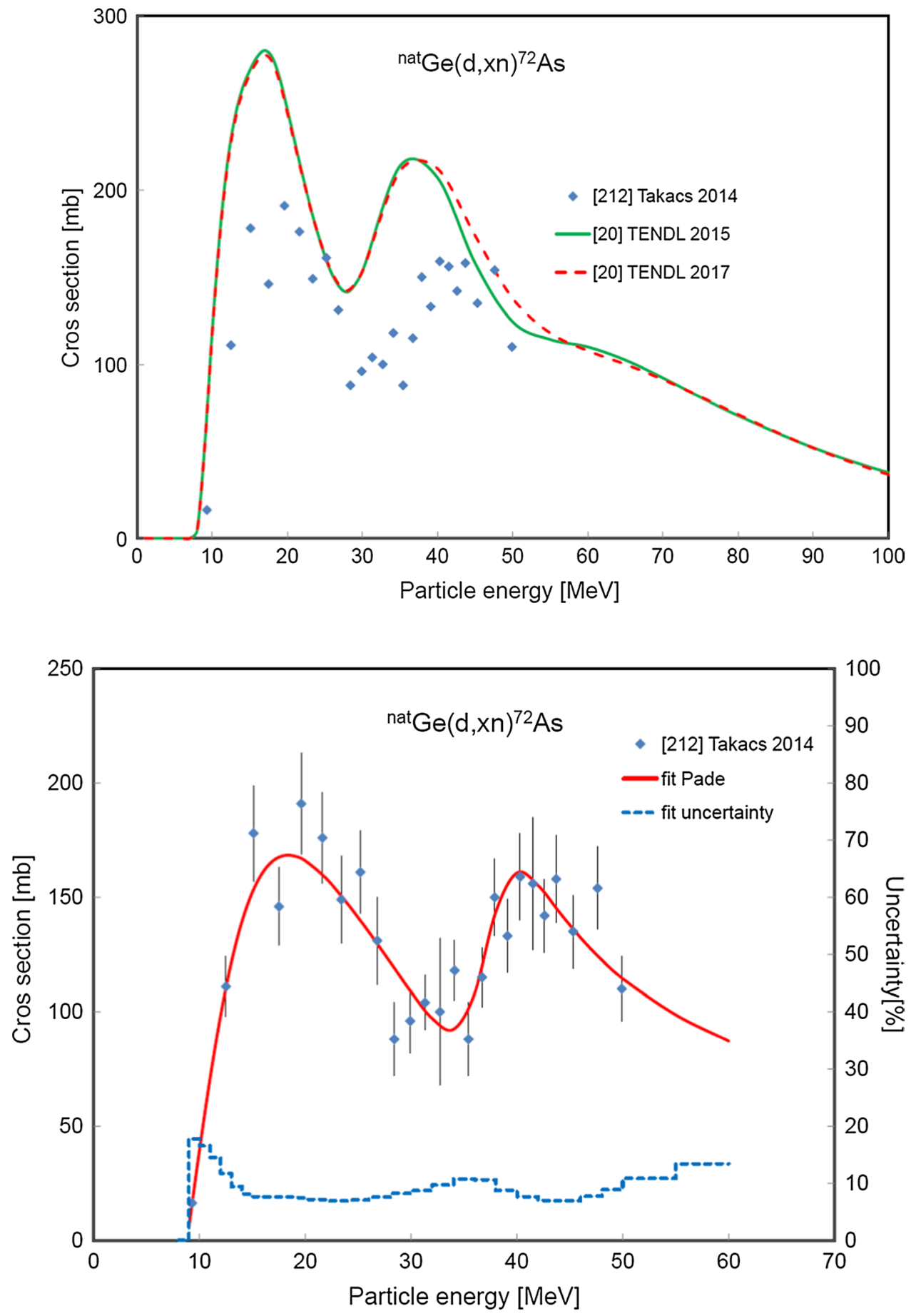
Thick target yields for production of ${ }^{72} \mathrm{As}$, and ${ }^{72}$ Se parent

See Figs. 79 and 80.

Fig. 79 Thick target yields calculated from the recommended cross sections for the ${ }^{\text {nat }} \mathrm{Ge}(\mathrm{p}, \mathrm{xn})^{72} \mathrm{As}$ and

${ }^{\text {nat }} \mathrm{Ge}(\mathrm{d}, \mathrm{xn}){ }^{72}$ As reactions

Fig. 80 Thick target yields calculated from the recommended cross sections for the ${ }^{75} \mathrm{As}(\mathrm{p}, 4 \mathrm{n})^{72} \mathrm{Se}$ and

${ }^{n a t} \operatorname{Br}(\mathrm{p}, \mathrm{x}){ }^{72} \mathrm{Se}$ reactions
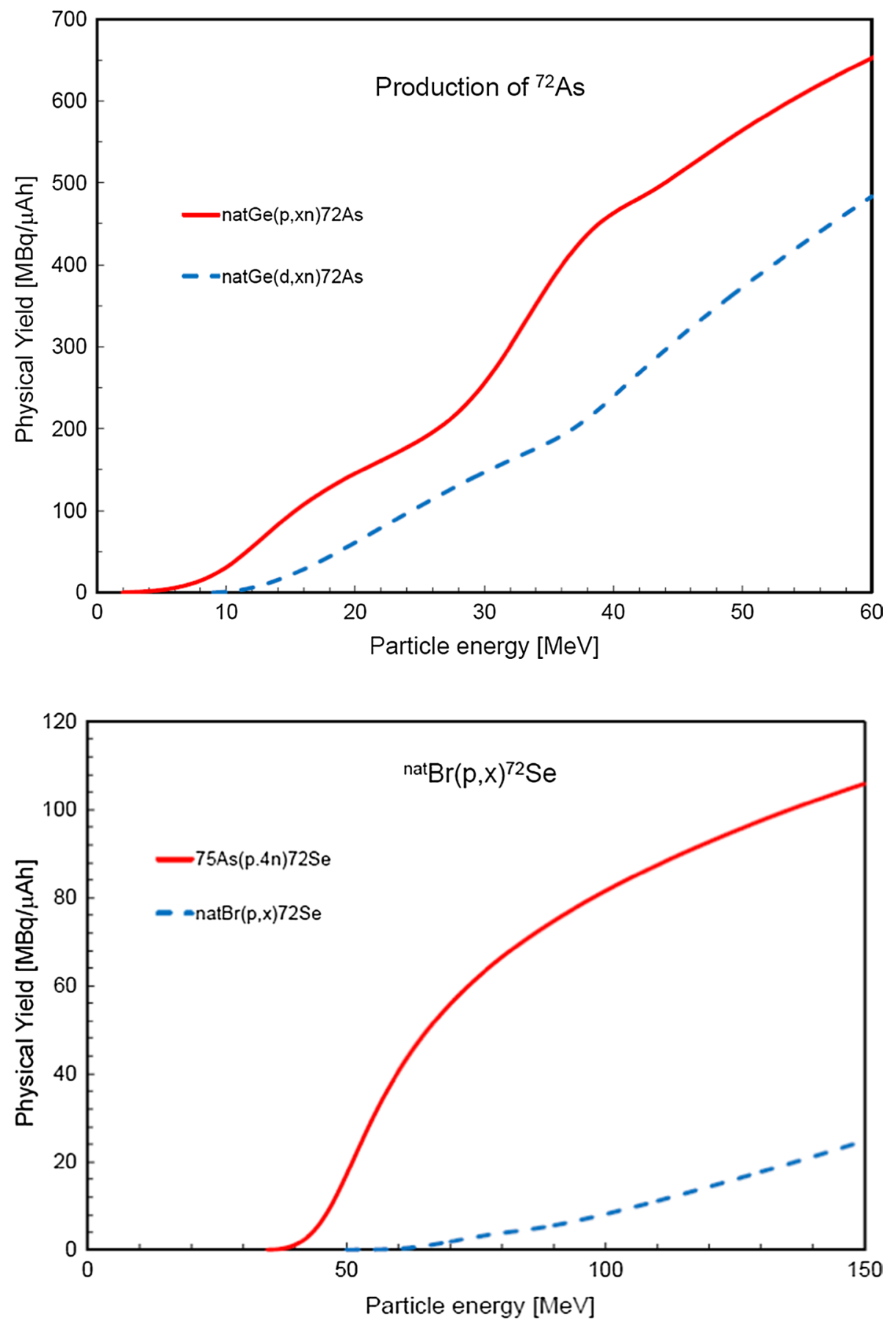


\section{Production of ${ }^{73} \mathrm{Se}\left(T_{1 / 2}=7.15 \mathrm{~h}\right)$}

Applications: ${ }^{73} \mathrm{Se}\left(T_{1 / 2}=7.15 \mathrm{~h} ; \mathrm{EC}=34.6 \%, \beta^{+}=65.4 \%\right.$; $\left.E_{\beta+}(\max )=1.65 \mathrm{MeV}\right)$ is an interesting $\beta^{+}$-emitting analogue of sulphur suitable for the imaging of enzymatic systems or sulphur-containing amino acids.

${ }^{73} \mathrm{Se}(7.15 \mathrm{~h}): \beta^{+}(65.4 \%)$, and $E_{\gamma}(\mathrm{keV})\left(P_{\gamma}(\%)\right): 67.07(70)$, 361.2 (97.0).

Evaluations have been made of the ${ }^{75} \mathrm{As}(\mathrm{p}, 3 \mathrm{n}){ }^{73} \mathrm{Se}$ and ${ }^{72} \mathrm{Ge}(\alpha, 3 \mathrm{n}){ }^{73} \mathrm{Se}$ direct production routes.

\section{${ }^{75} \mathrm{As}(\mathrm{p}, 3 \mathrm{n}){ }^{73} \mathrm{Se}$}

The four experimental datasets available in the literature are shown in Fig. 81 [36, 206, 213, 214] together with the TENDL calculations. One dataset was rejected (Mushtaq et al. [206] (values too low near maximum)), and the remaining three sets were used in the statistical fitting procedure. The selected data and their experimental uncertainties are shown in Fig. 82 together with the Padé $(L=9, N=64$,
Fig. 81 Four experimental reaction available in the literature [36, 206, 213, 214], and TENDL calculations datasets for the ${ }^{75} \mathrm{As}(\mathrm{p}, 3 \mathrm{n}){ }^{73} \mathrm{Se}$

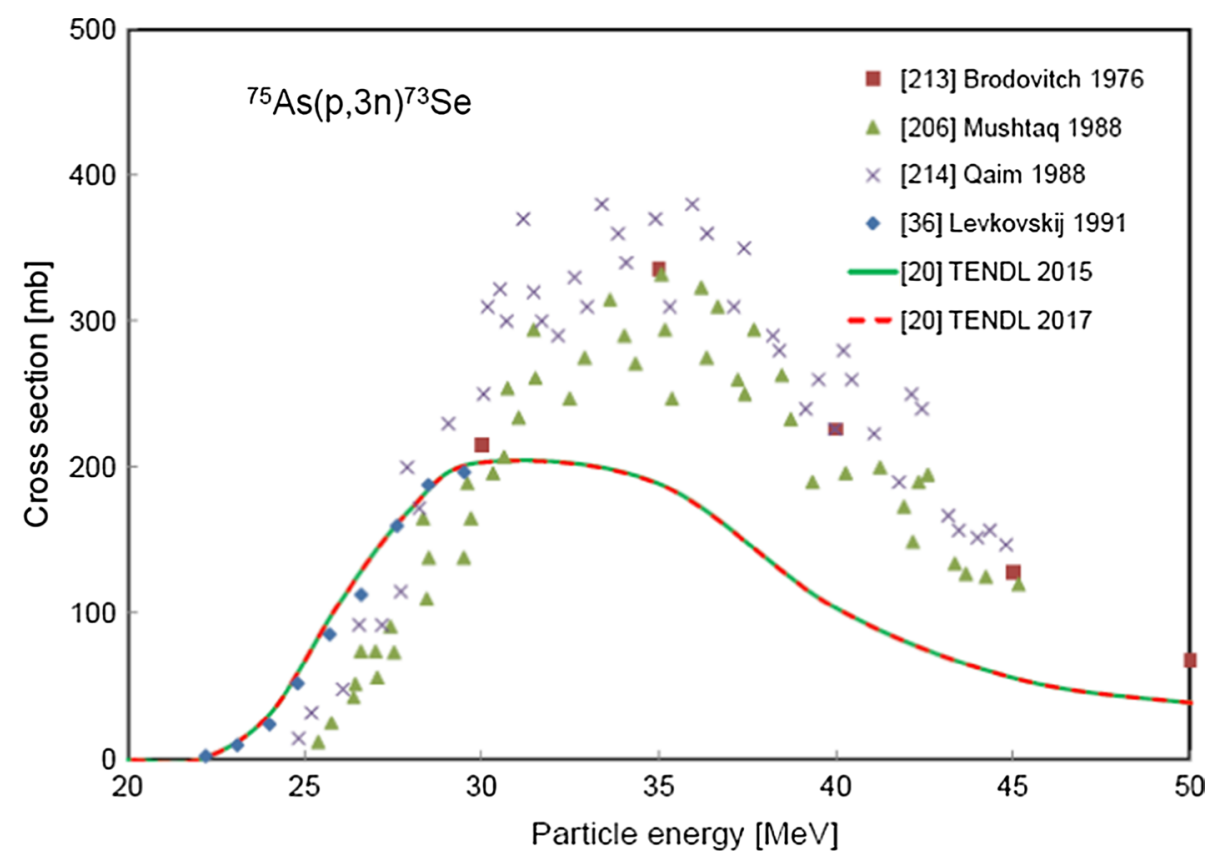

Fig. 82 Three selected experimental datasets for the ${ }^{75} \mathrm{As}(\mathrm{p}, 3 \mathrm{n}){ }^{73} \mathrm{Se}$ reaction $[36$, 213, 214] with the Padé fit $\left(L=9, N=64, \chi^{2}=1.52\right.$, solid line) and estimated total uncertainties in percentages, including $4 \%$ systematic uncertainty (dashed line, right-hand scale)

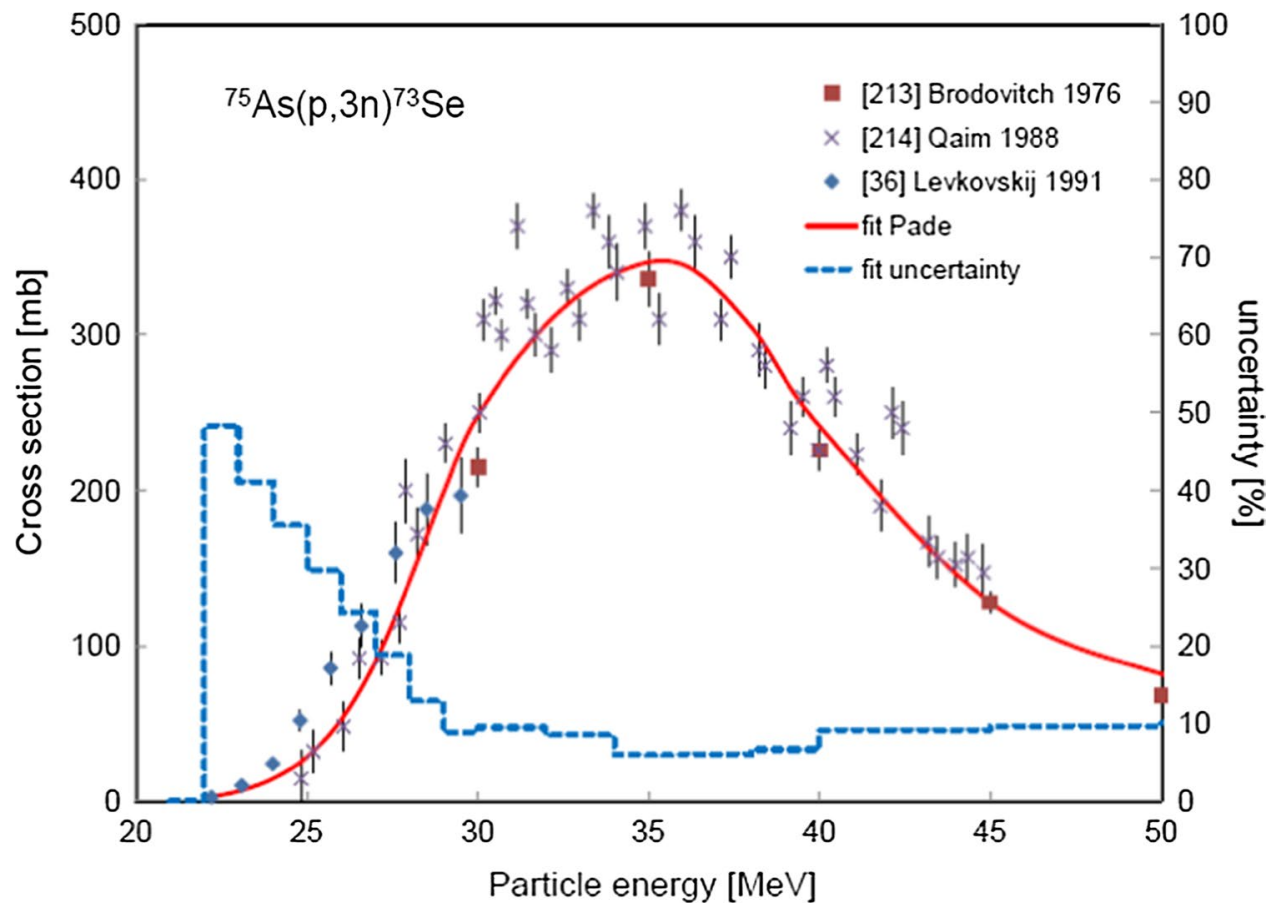


$\left.\chi^{2}=1.52\right)$ and estimated uncertainty in percentages, including $4 \%$ systematic uncertainty (right-hand scale).

\section{${ }^{72} \mathrm{Ge}(a, 3 n){ }^{73} \mathrm{Se}$}

The two experimental datasets available in the literature are shown in Fig. 83 [36, 215] together with the TENDL calculations. Both datasets were used in the statistical fitting procedure. The data and their experimental uncertainties are shown in Fig. 84 together with the Padé fit $(L=8, N=27$, $\left.\chi^{2}=3.81\right)$ and estimated uncertainty in percentages, including $4 \%$ systematic uncertainty (right-hand scale).
Fig. 83 Two experimental datasets for the ${ }^{72} \mathrm{Ge}(\alpha, 3 \mathrm{n}){ }^{73} \mathrm{Se}$ reaction available in the literature [36, 215], and TENDL calculations

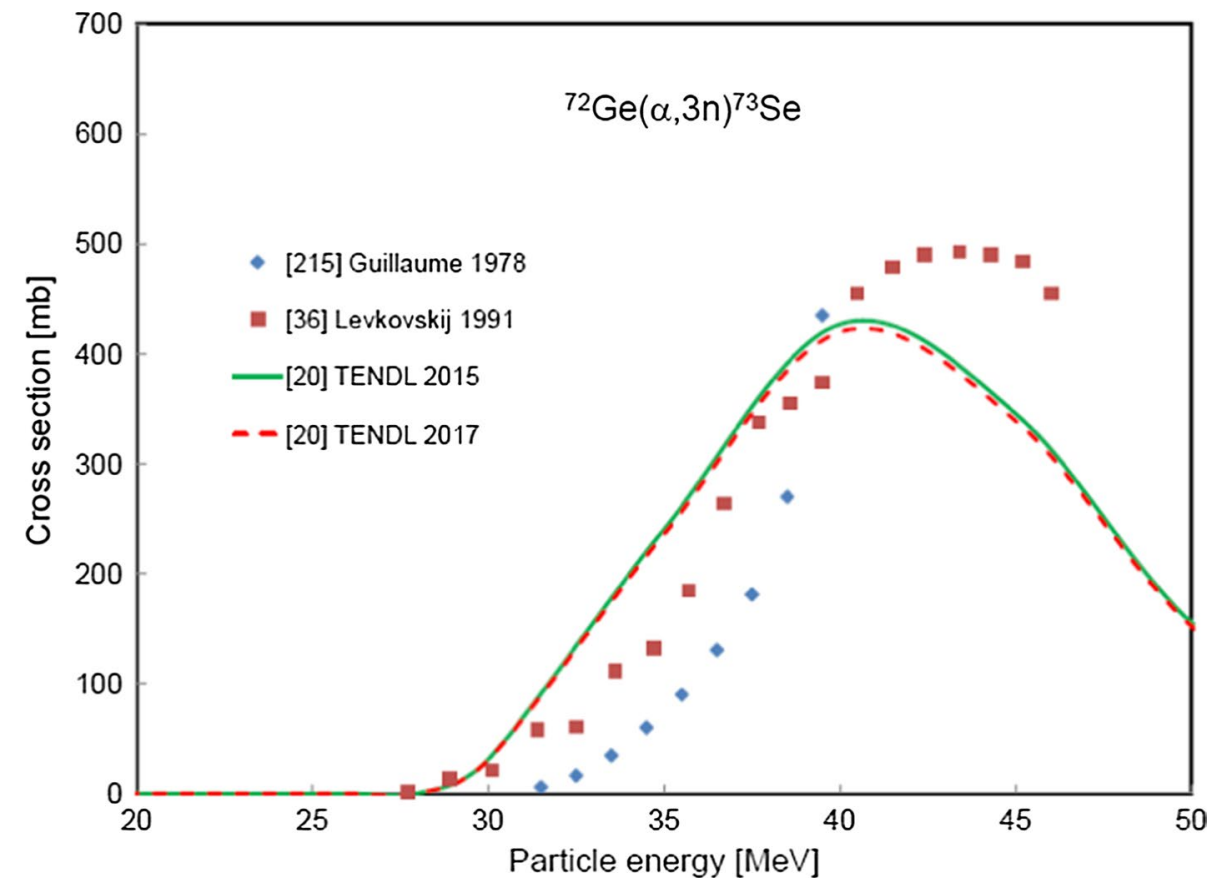

Fig. 84 Two experimental datasets for the ${ }^{72} \mathrm{Ge}(\alpha, 3 \mathrm{n}){ }^{73} \mathrm{Se}$ reaction $[36,215]$ with the Padé fit $\left(L=8, N=27, \chi^{2}=3.81\right.$, solid line) and estimated total uncertainties in percentages, including 4\% systematic uncertainty (dashed line, right-hand scale)

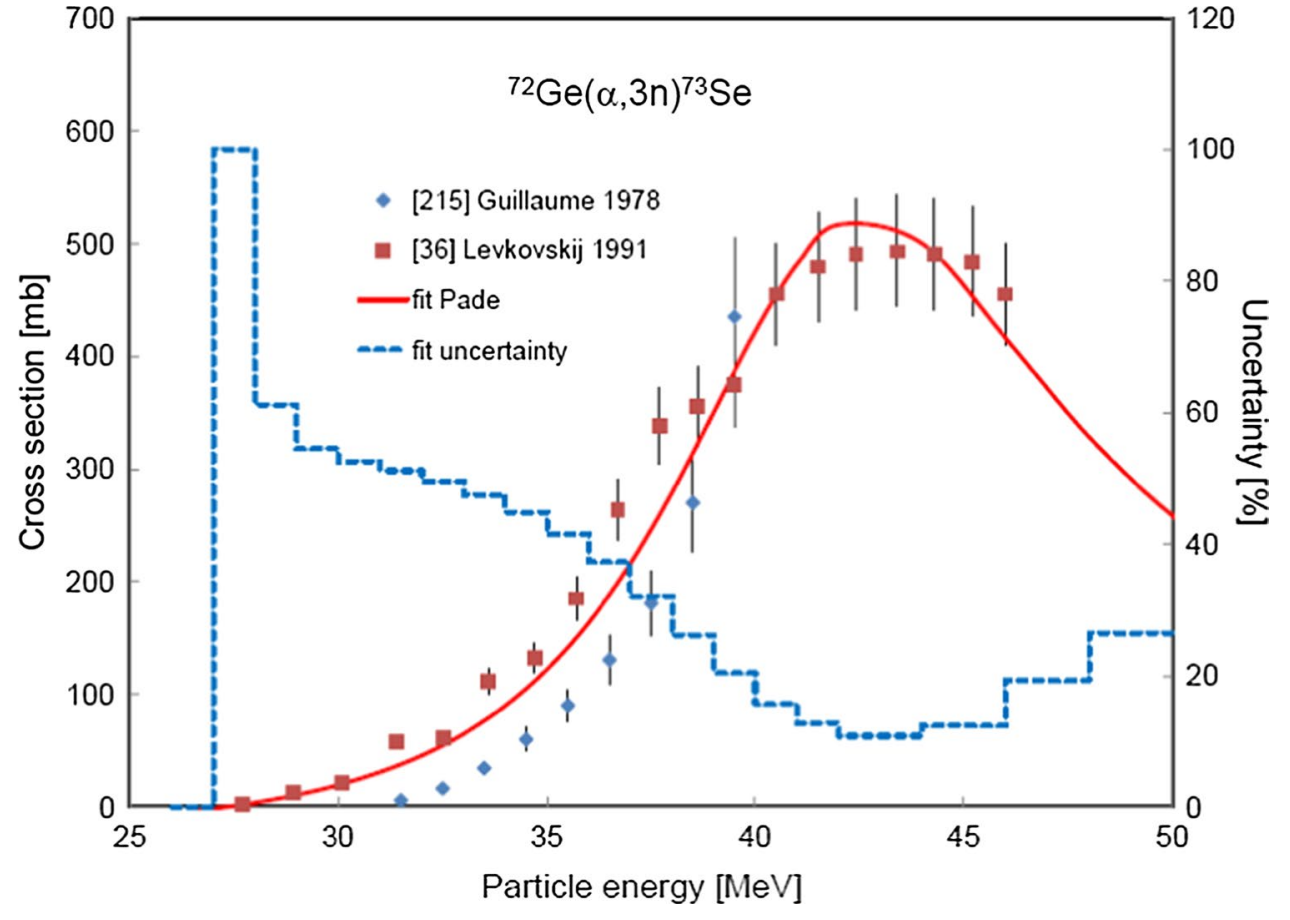


Thick target yields for production of ${ }^{73} \mathrm{Se}$

See Fig. 85.

Fig. 85 Thick target yields calculated from the recommended cross sections for the ${ }^{75} \mathrm{As}(\mathrm{p}, 3 \mathrm{n}){ }^{73} \mathrm{Se}$ and

${ }^{72} \mathrm{Ge}(\alpha, 3 \mathrm{n}){ }^{73} \mathrm{Se}$ reactions

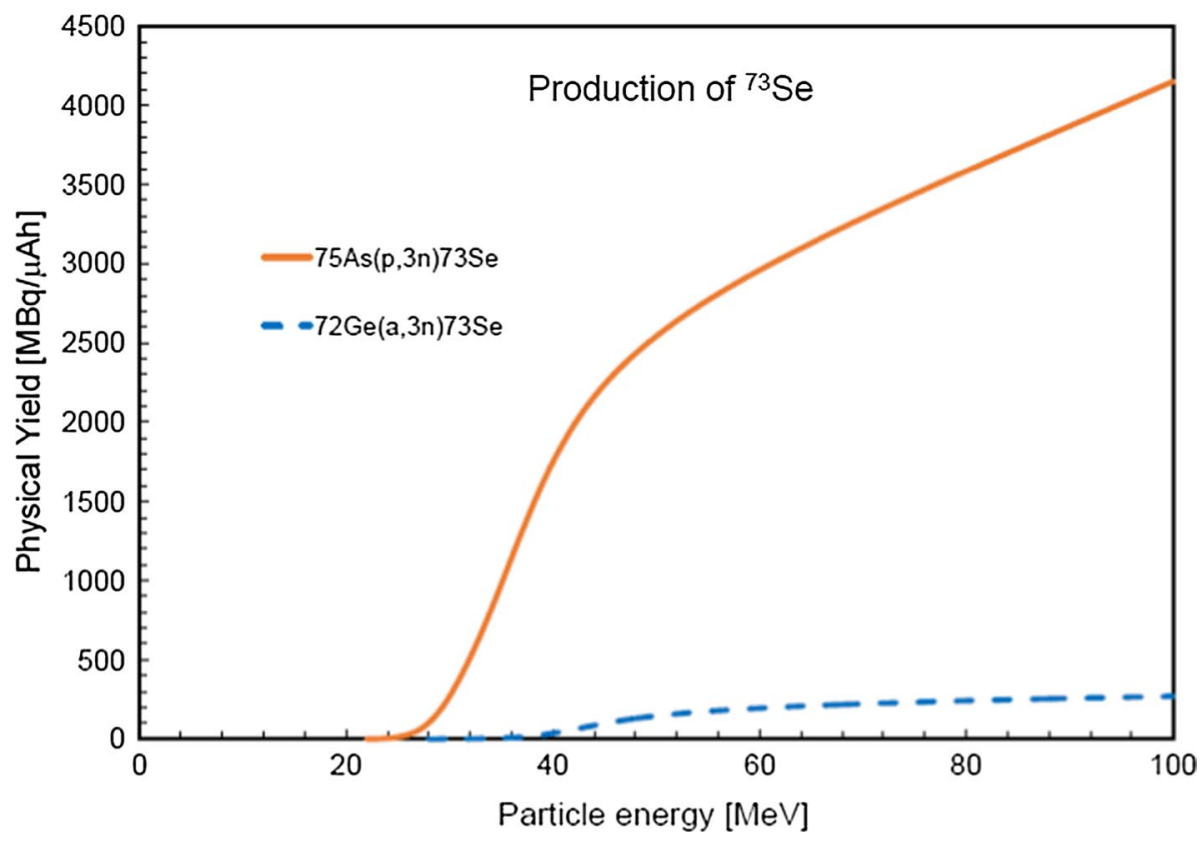




\section{Production of ${ }^{76} \mathrm{Br}\left(T_{1 / 2}=16.2 \mathrm{~h}\right)$}

Applications: Longer-lived positron-emitting radiohalogens were some of the first radionuclides studied to follow processes with kinetics inappropriate for ${ }^{18} \mathrm{~F}$ application. ${ }^{76} \mathrm{Br}$ was used in several studies to label monoclonal antibodies, although the large number of accompanying gamma rays that result in a relatively high radiation burden and poor imaging properties has seen a subsequent decline of interest in this radionuclide.

${ }^{76} \mathrm{Br}(16.2 \mathrm{~h}): \beta^{+}(55 \%)$ and $E_{\gamma}(\mathrm{keV})\left(P_{\gamma}(\%)\right): 559.09$ (74), 657.02 (15.9), 1853.67 (14.7).

Evaluations have been made of the ${ }^{76} \mathrm{Se}(\mathrm{p}, \mathrm{n}){ }^{76} \mathrm{Br}$,

${ }^{77} \mathrm{Se}(\mathrm{p}, 2 \mathrm{n}){ }^{76} \mathrm{Br}$ and ${ }^{75} \mathrm{As}(\alpha, 3 \mathrm{n}){ }^{76} \mathrm{Br}$ production routes.

\section{${ }^{76} \mathrm{Se}(p, n){ }^{76} \mathrm{Br}$}

The five experimental datasets available in the literature are shown in Fig. 86 [36, 216-219] together with the TENDL calculations. Two datasets were rejected (Kovács et al. [218] (values too low near maximum), and Hassan et al. [219] (discrepant data near maximum)), and the remaining three sets were used in the statistical fitting procedure. The selected data and their experimental uncertainties are shown in Fig. 87 together with the Padé fit $\left(L=8, N=39, \chi^{2}=1.05\right)$ and estimated uncertainty in percentages, including $4 \%$ systematic uncertainty (right-hand scale).
Fig. 86 Five experimental datasets for the ${ }^{76} \mathrm{Se}(\mathrm{p}, \mathrm{n}){ }^{76} \mathrm{Br}$ reaction available in the literature [36, 216-219], and TENDL calculations

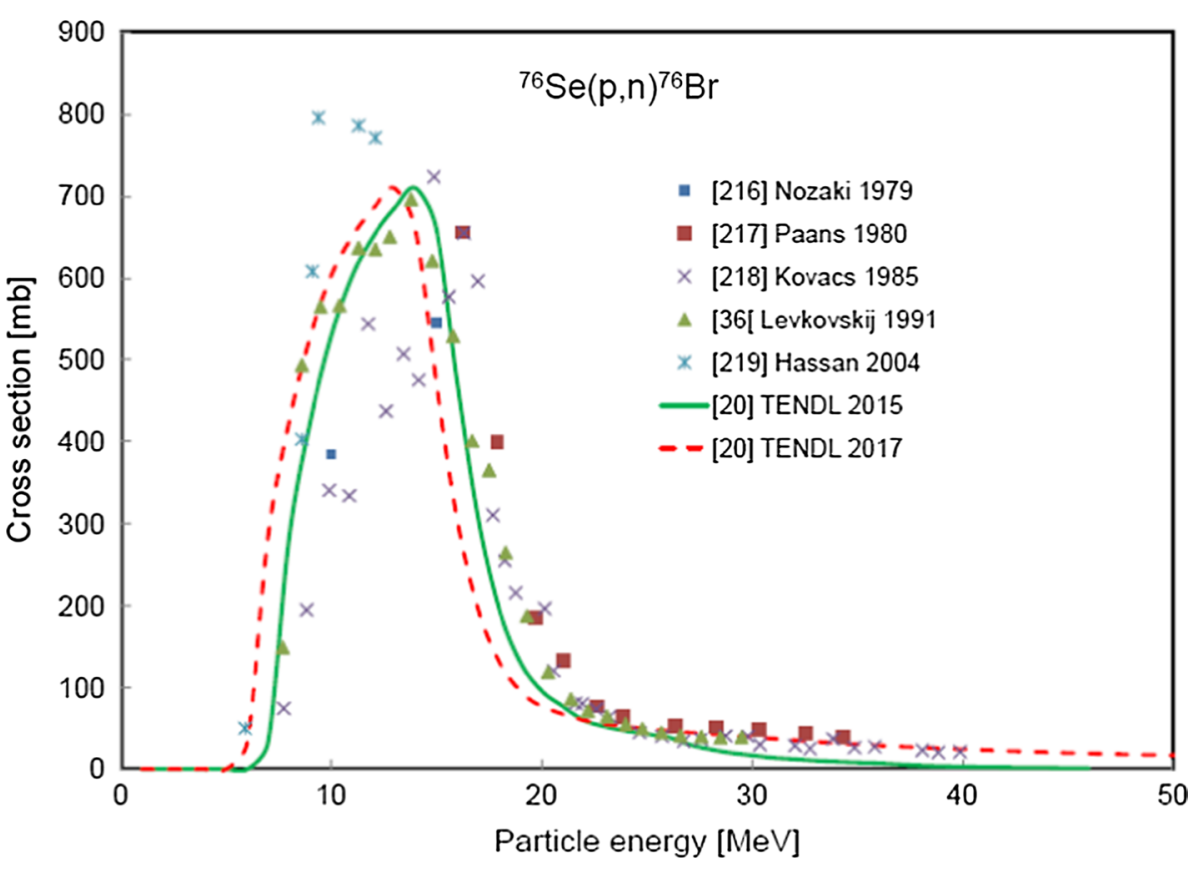


Fig. 87 Three selected experimental datasets for the ${ }^{76} \mathrm{Se}(\mathrm{p}, \mathrm{n}){ }^{76} \mathrm{Br}$ reaction $[36,216$, 217] with the Padé fit $(L=8$, $N=39, \chi^{2}=1.05$, solid line) and estimated total uncertainties in percentages, including $4 \%$ systematic uncertainty (dashed line, right-hand scale)

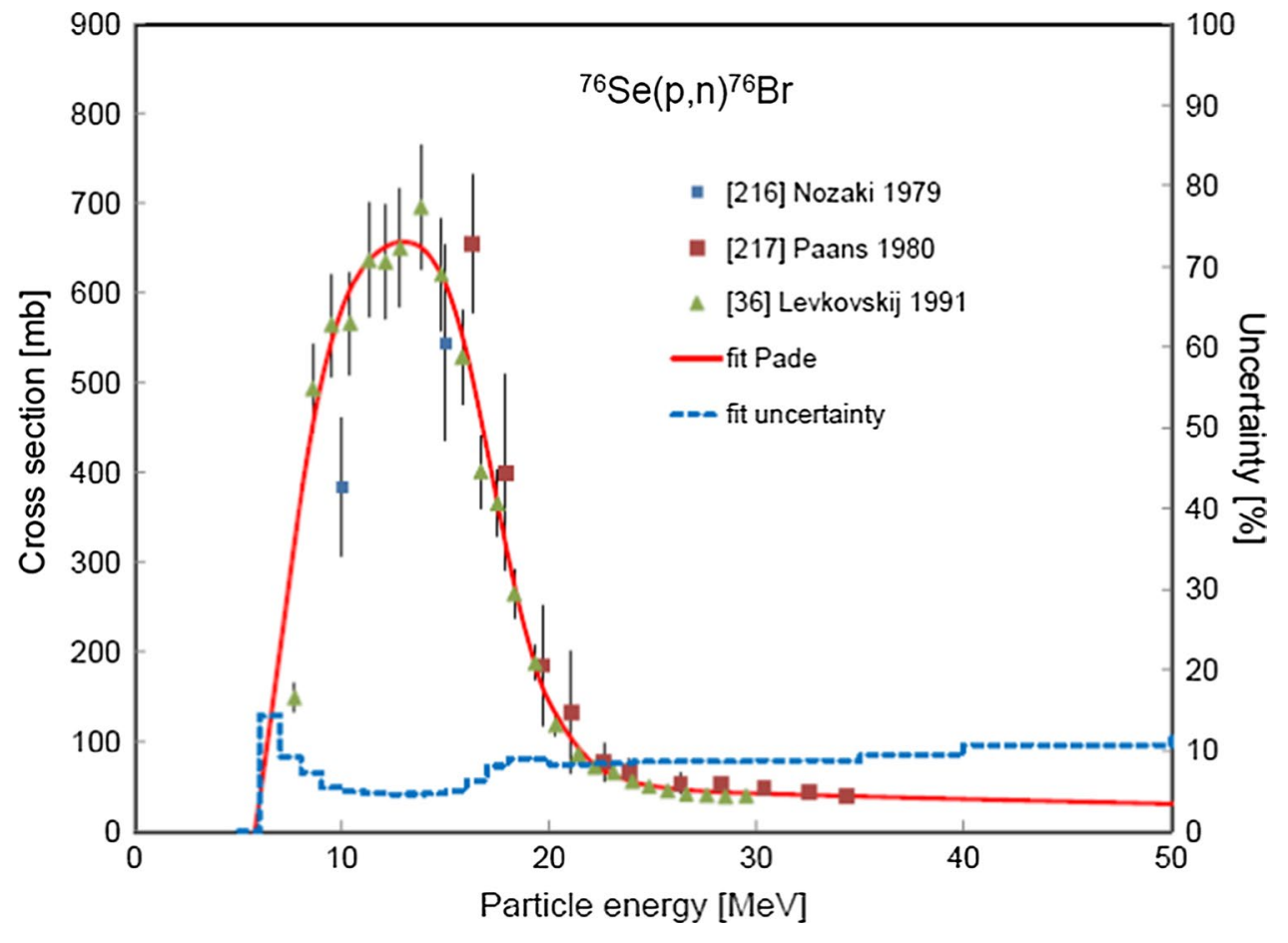




\section{${ }^{77} \mathrm{Se}(p, 2 n){ }^{76} \mathrm{Br}$}

The four experimental datasets available in the literature are shown in Fig. 88 [36, 219-221] together with the TENDL calculations. Two datasets were rejected (Janssen et al. [220] (values too low), and Hassan et al. [219] (values too high)), and the remaining two sets were used in the statistical fitting procedure. The selected data and their experimental uncertainties are shown in Fig. 89 together with the Padé fit $(L=9$, $\left.N=52, \chi^{2}=1.34\right)$ and estimated uncertainty in percentages, including $4 \%$ systematic uncertainty (right-hand scale).
Fig. 88 Four experimental datasets for the ${ }^{77} \mathrm{Se}(\mathrm{p}, 2 \mathrm{n}){ }^{76} \mathrm{Br}$ reaction available in the literature [36, 219-221], and TENDL calculations
Fig. 89 Two selected experimental datasets for the ${ }^{77} \mathrm{Se}(\mathrm{p}, 2 \mathrm{n}){ }^{76} \mathrm{Br}$ reaction $[36$, 221] with the Padé fit $(L=9$, $N=52, \chi^{2}=1.34$, solid line) and estimated total uncertainties in percentages, including $4 \%$ systematic uncertainty (dashed line, right-hand scale)
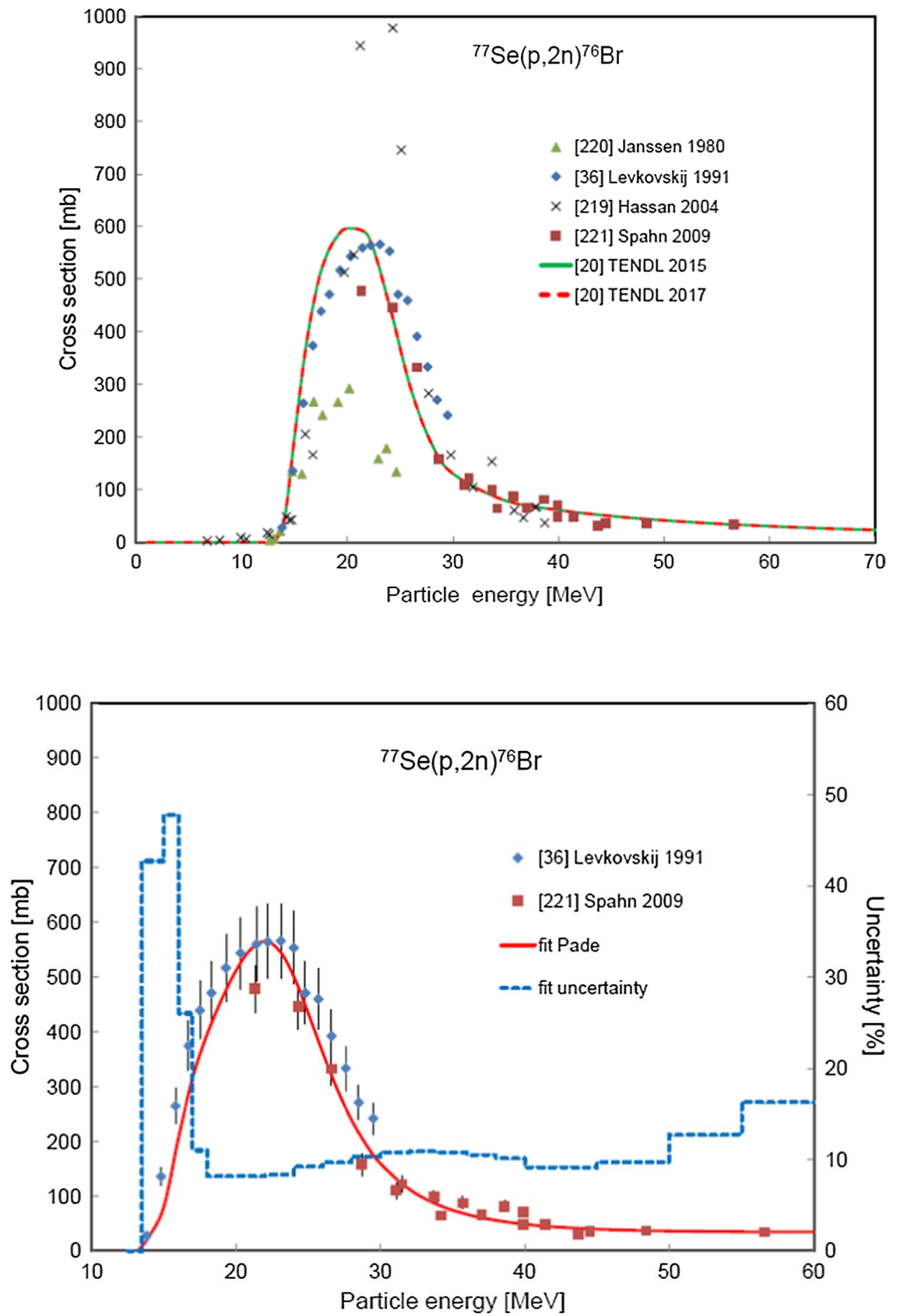


\section{${ }^{75} \mathrm{As}(a, 3 n){ }^{76} \mathrm{Br}$}

The five experimental datasets available in the literature are shown in Fig. 90 [216, 217, 222-224] together with the TENDL calculations. All datasets were used for the statistical fitting procedure. The data and their experimental uncertainties are shown in Fig. 91 together with the Padé fit ( $L=10, N=70, \chi^{2}=2.43$ ) and estimated uncertainty in percentages, including $4 \%$ systematic uncertainty (right-hand scale). An additional dataset published after the evaluation cut-off date was also included in the fit (Breunig et al. [224]) and is shown in Fig. 90.
Fig. 90 Five experimental datasets for the ${ }^{75} \mathrm{As}(\alpha, 3 \mathrm{n})^{76} \mathrm{Br}$ reaction available in the literature [216, 217, 222-224], and TENDL calculations

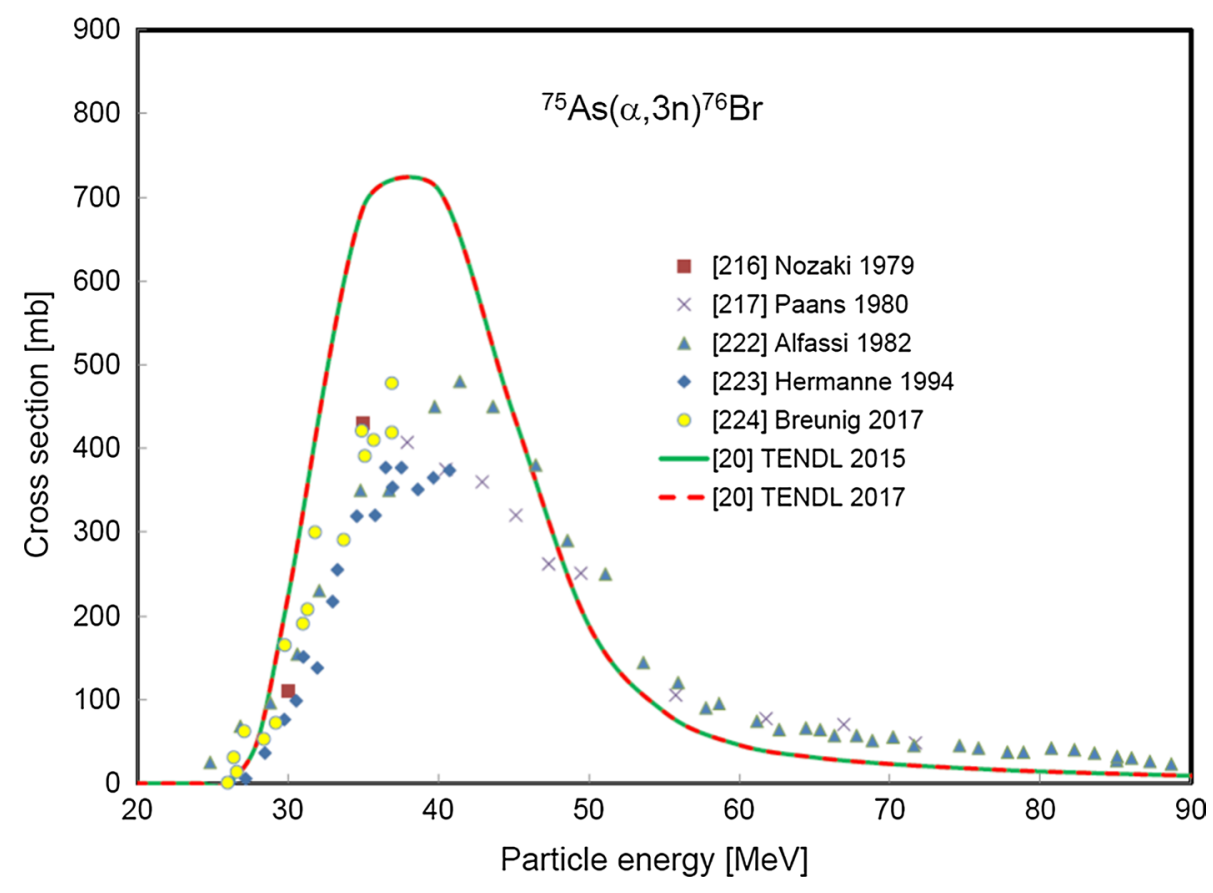

Fig. 91 Five experimental datasets for the ${ }^{75} \mathrm{As}(\alpha, 3 \mathrm{n}){ }^{76} \mathrm{Br}$ reaction [216, 217, 222-224] with the Padé fit $(L=10, N=70$, $\chi^{2}=2.43$, solid line) and estimated total uncertainties in percentages, including $4 \%$ systematic uncertainty (dashed line, right-hand scale)

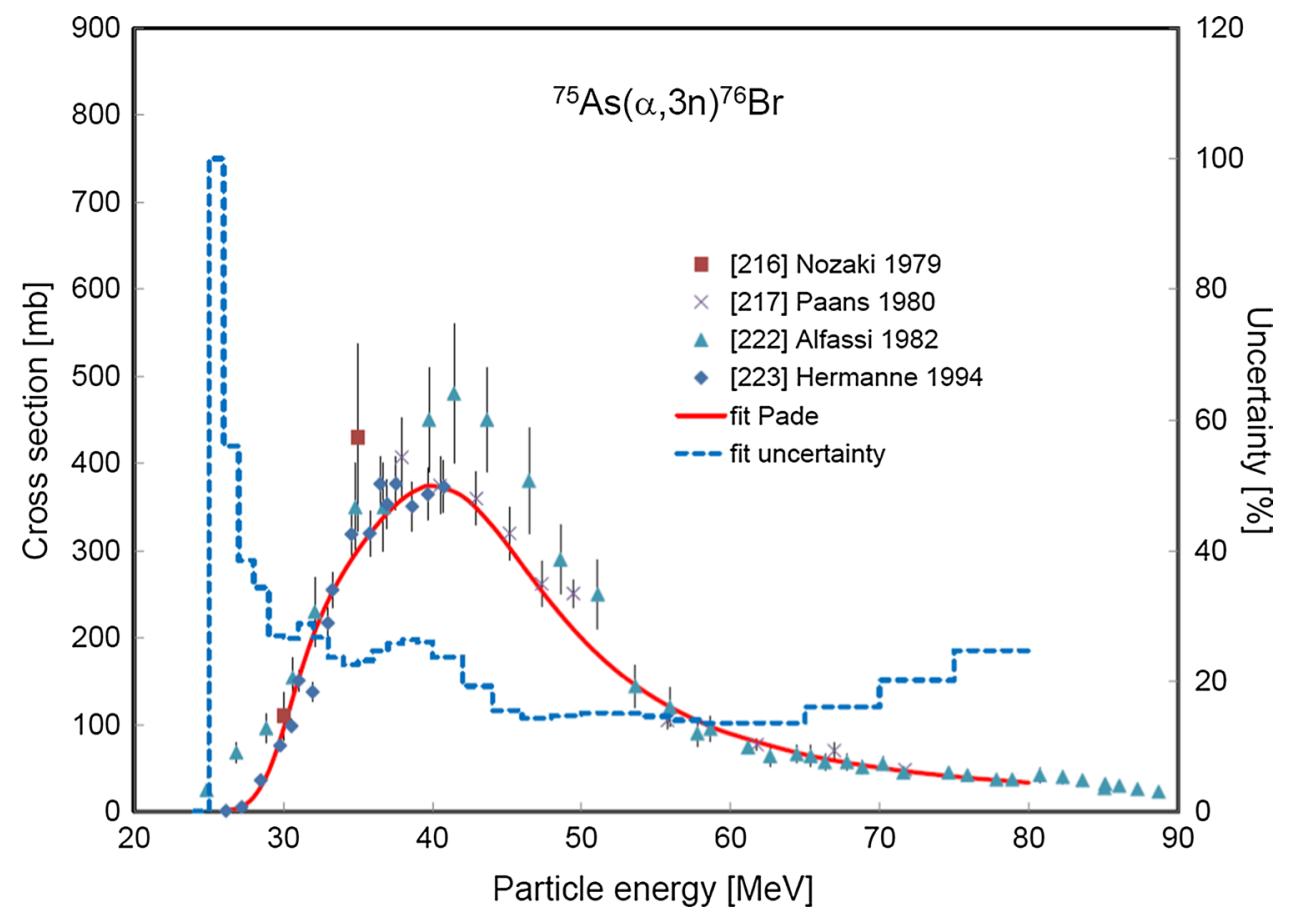


Thick target yields for ${ }^{76} \mathrm{Br}$

See Fig. 92.

Fig. 92 Thick target yields calculated from the recommended cross sections for the

${ }^{76} \mathrm{Se}(\mathrm{p}, \mathrm{n})^{76} \mathrm{Br},{ }^{77} \mathrm{Se}(\mathrm{p}, 2 \mathrm{n}){ }^{76} \mathrm{Br}$ and ${ }^{75} \mathrm{As}(\alpha, 3 \mathrm{n}){ }^{76} \mathrm{Br}$ reactions

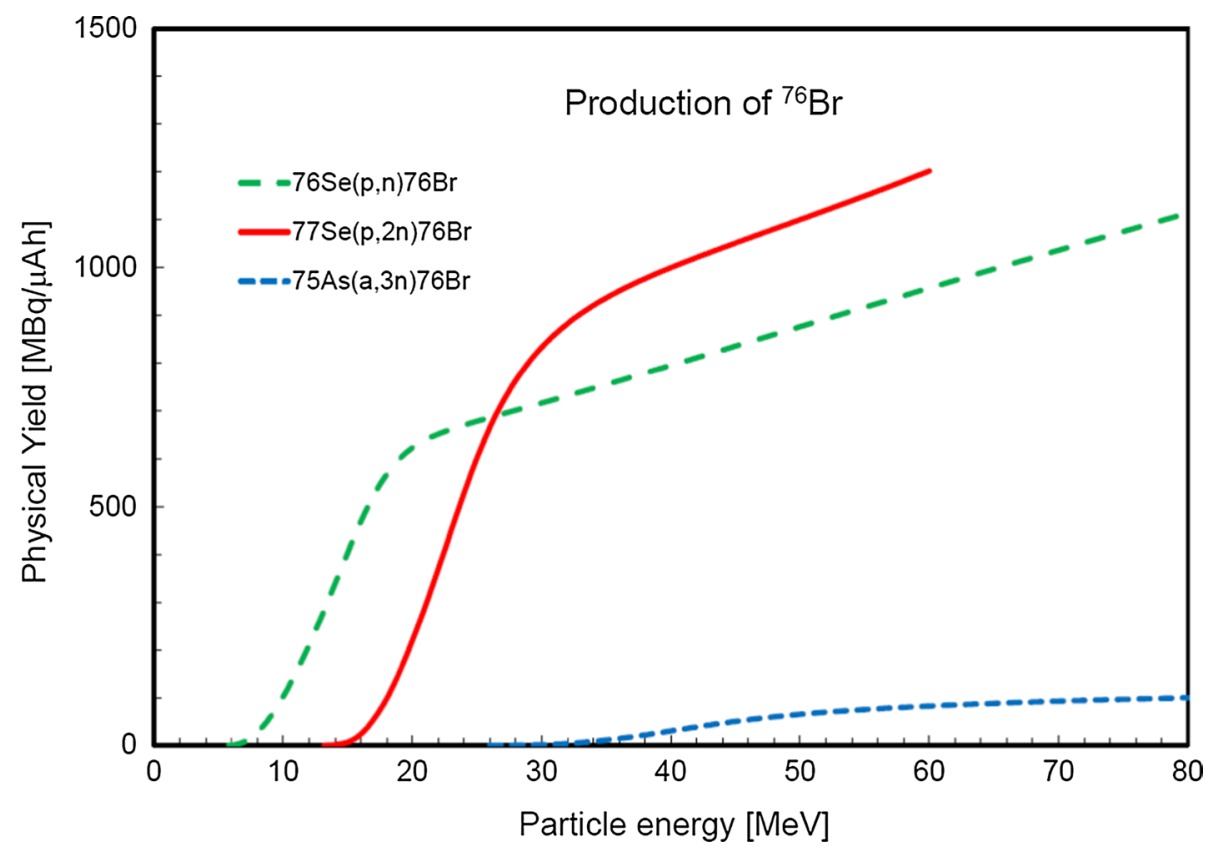




\section{Production of ${ }^{82} \mathrm{Sr}$ parent $\left(T_{1 / 2}=25.35 \mathrm{~d}\right)$ of short-lived ${ }^{82} \mathrm{Rb}\left(T_{1 / 2}=1.2575 \mathrm{~min}\right)$}

Applications: Generator-produced ${ }^{82} \mathrm{Rb}$ is widely used in myocardial perfusion imaging, particularly in the USA. This isotope undergoes rapid uptake by myocardiocytes, and therefore is a valuable tool for identifying myocardial ischemia by means of PET. Such a short half-life allows one to perform both stress and rest perfusion studies within $30 \mathrm{~min}$.

${ }^{82} \mathrm{Rb}(1.2575 \mathrm{~min}): \beta^{+}(95.43 \%)$, and $E_{\gamma}(\mathrm{keV})\left(P_{\gamma}(\%)\right)$ : 776.52 (15.08).

${ }^{82} \mathrm{Sr}(25.35 d)$ : detected by means of radiation emitted by daughter ${ }^{82} \mathrm{Rb}$.
Evaluations have been undertaken of the ${ }^{\text {nat }} \mathrm{Rb}(\mathrm{p}, \mathrm{xn}){ }^{82} \mathrm{Sr}$ and ${ }^{85} \mathrm{Rb}(\mathrm{p}, 4 \mathrm{n}){ }^{82} \mathrm{Sr}$ parent production routes.

${ }^{\text {nat }} \mathrm{Rb}(\mathrm{p}, \mathrm{xn}){ }^{82} \mathrm{Sr}$

The seven experimental datasets available in the literature are shown in Fig. 93 [138, 225-230] together with the TENDL calculations. Two datasets were rejected (Horiguchi et al. [225] (values too high), and Deptula et al. [226] (values too high)), and the remaining five sets were used in the statistical fitting procedure. The selected data and their experimental uncertainties are shown in Fig. 94 together with the Padé fit $\left(L=13, N=49, \chi^{2}=1.15\right)$ and estimated uncertainty in percentages, including $4 \%$ systematic uncertainty (right-hand scale).
Fig. 93 Seven experimental datasets for the ${ }^{\text {nat }} \mathrm{Rb}(\mathrm{p}, \mathrm{xn})^{82} \mathrm{Sr}$ reaction available in the literature [138, 225-230], and TENDL calculations

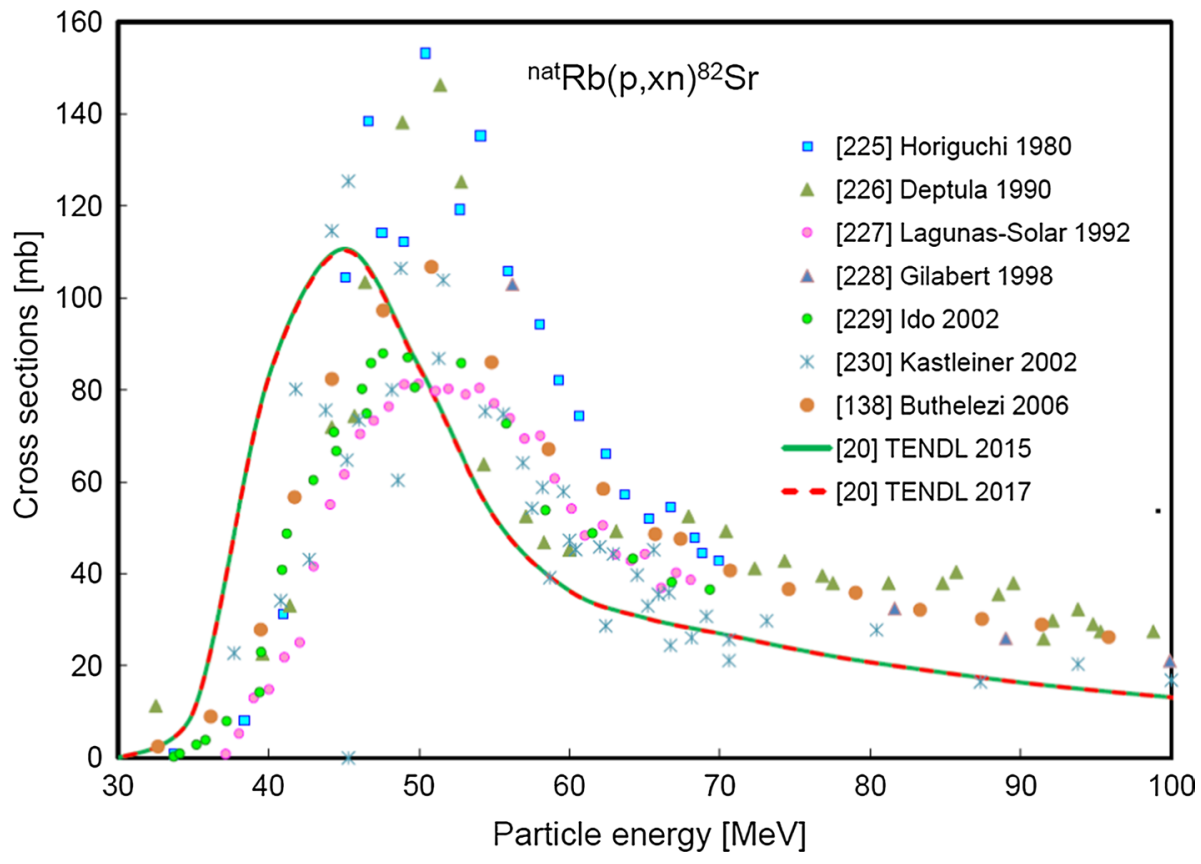


Fig. 94 Five selected experimental datasets for the ${ }^{n a t} \mathrm{Rb}(\mathrm{p}, \mathrm{xn}){ }^{82} \mathrm{Sr}$ reaction [138, 227-230] with the Padé fit $\left(L=13, N=49, \chi^{2}=1.15\right.$, solid line) and estimated total uncertainties in percentages, including $4 \%$ systematic uncertainty (dashed line, right-hand scale)

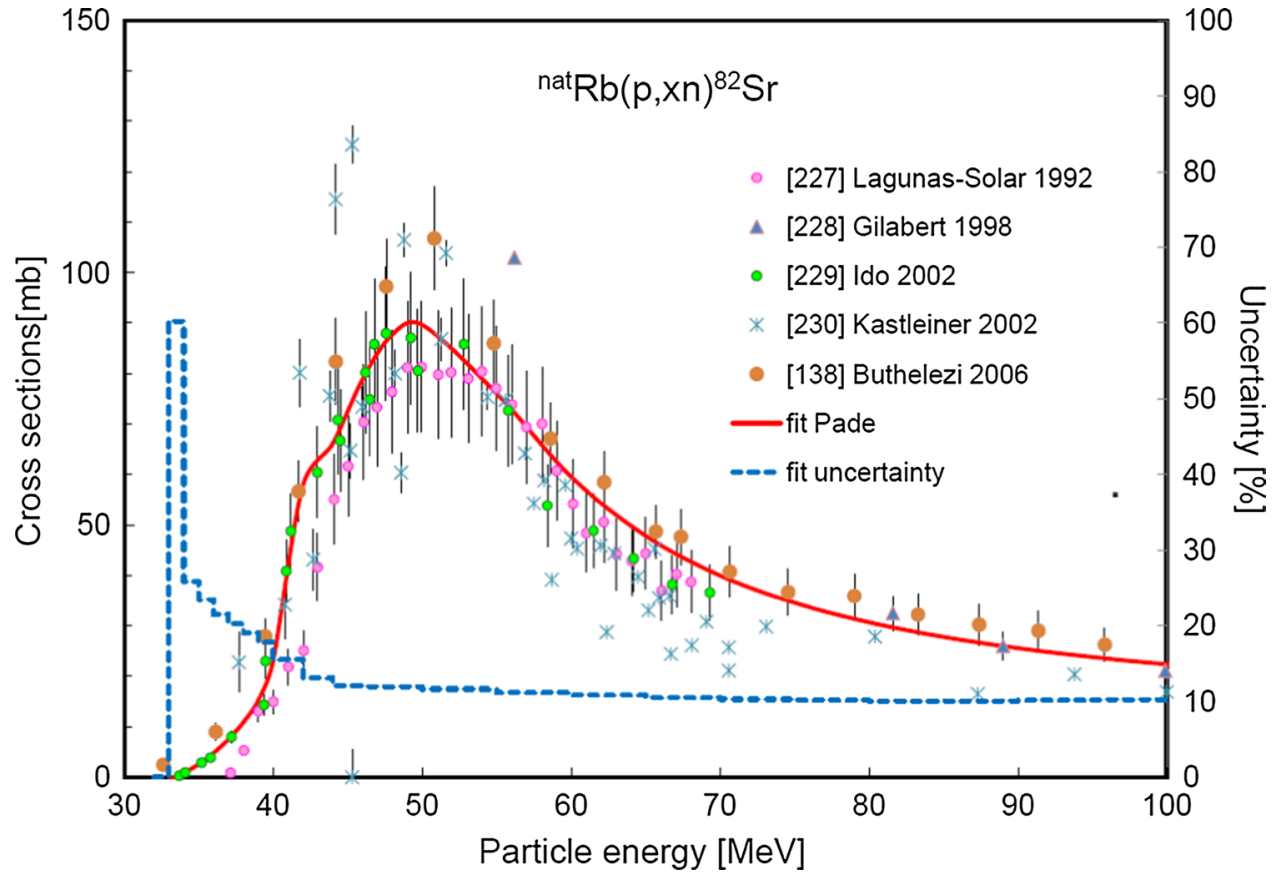




\section{${ }^{85} \mathrm{Rb}(\mathrm{p}, 4 \mathrm{n}){ }^{82} \mathrm{Sr}$}

The five experimental datasets available in the literature are shown in Fig. 95 [138, 225, 227, 229, 230] together with the TENDL calculations. All datasets were used in the statistical fitting procedure. The data and their experimental uncertainties are shown in Fig. 96 together with the Padé fit $\left(L=9, N=49, \chi^{2}=1.60\right)$ and estimated uncertainty in percentages, including $4 \%$ systematic uncertainty (right-hand scale).
Fig. 95 Five experimental datasets for the ${ }^{85} \mathrm{Rb}(\mathrm{p}, 4 \mathrm{n})^{82} \mathrm{Sr}$ reaction available in the literature [138, 225, 227, 229, 230], and TENDL calculations

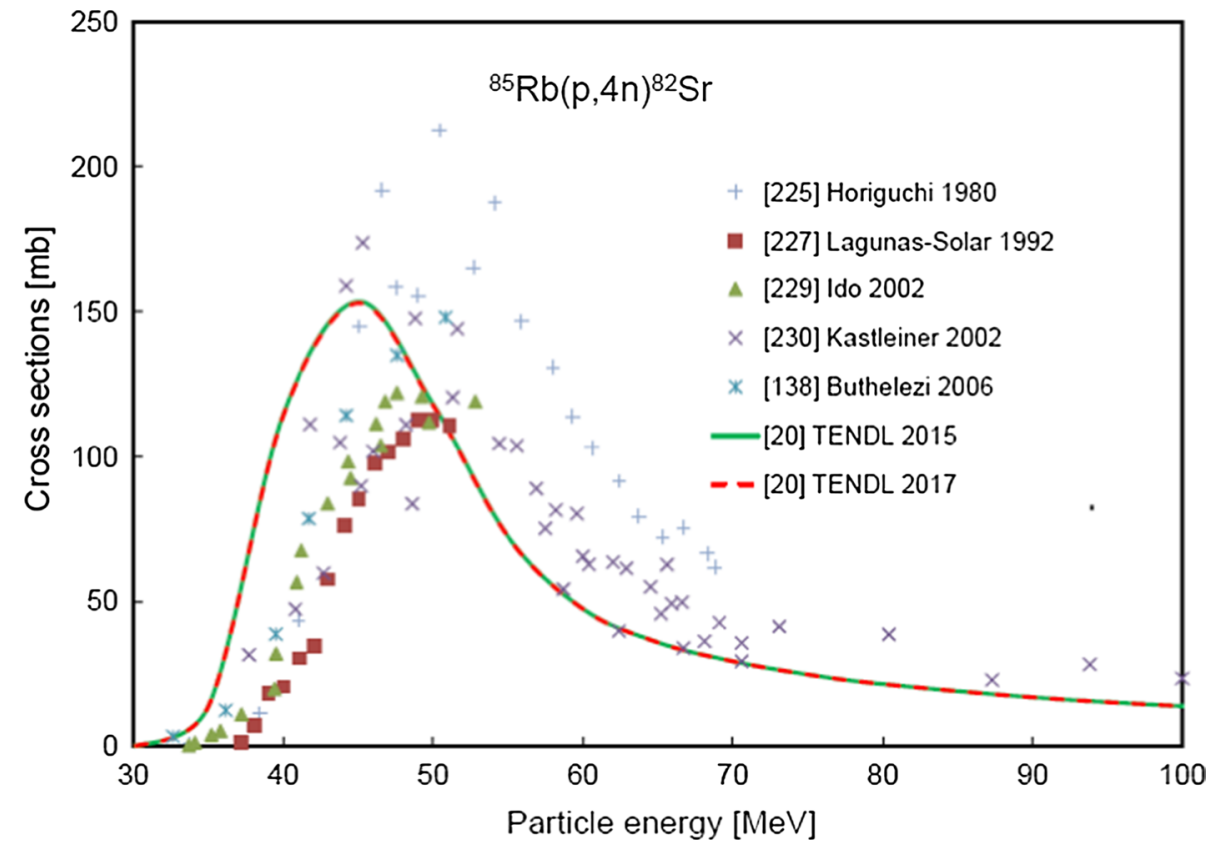

Fig. 96 Five experimental datasets for the ${ }^{85} \mathrm{Rb}(\mathrm{p}, 4 \mathrm{n}){ }^{82} \mathrm{Sr}$ reaction $[138,225,227,229$, 230] with the Padé fit $(L=9$, $N=49, \chi^{2}=1.60$, solid line) and estimated total uncertainties in percentages, including $4 \%$ systematic uncertainty (dashed line, right-hand scale)

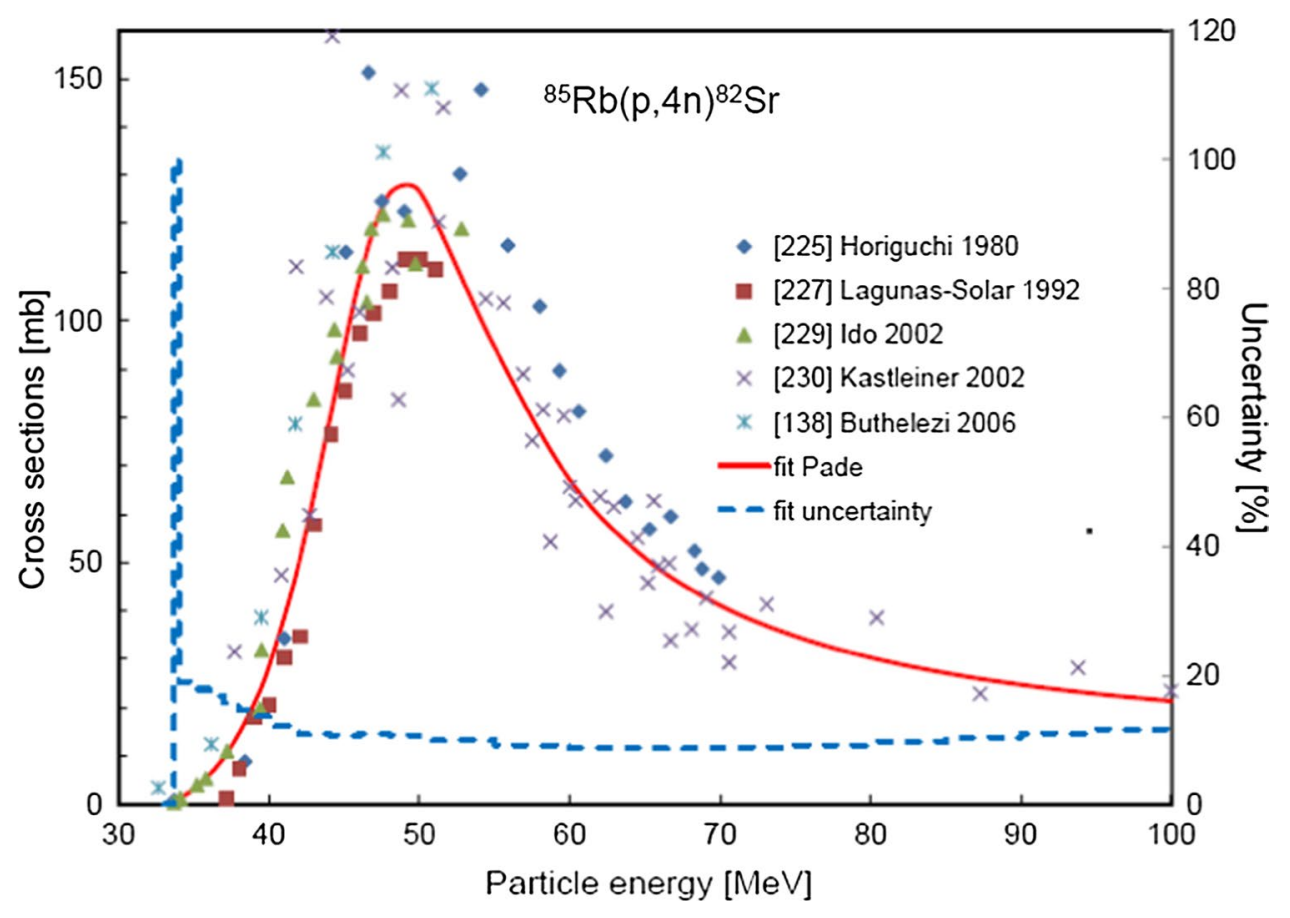


Thick target yields for ${ }^{82} \mathrm{Sr}$ parent of short-lived ${ }^{82} \mathrm{Rb}$

See Fig. 97.

Fig. 97 Thick target yields calculated from the recommended cross sections for the ${ }^{\text {nat }} \mathrm{Rb}(\mathrm{p}, \mathrm{xn})^{82} \mathrm{Sr}$ and

${ }^{85} \mathrm{Rb}(\mathrm{p}, 4 \mathrm{n}){ }^{82} \mathrm{Sr}$ reactions to produce long-lived parent for short-lived ${ }^{82} \mathrm{Rb}$

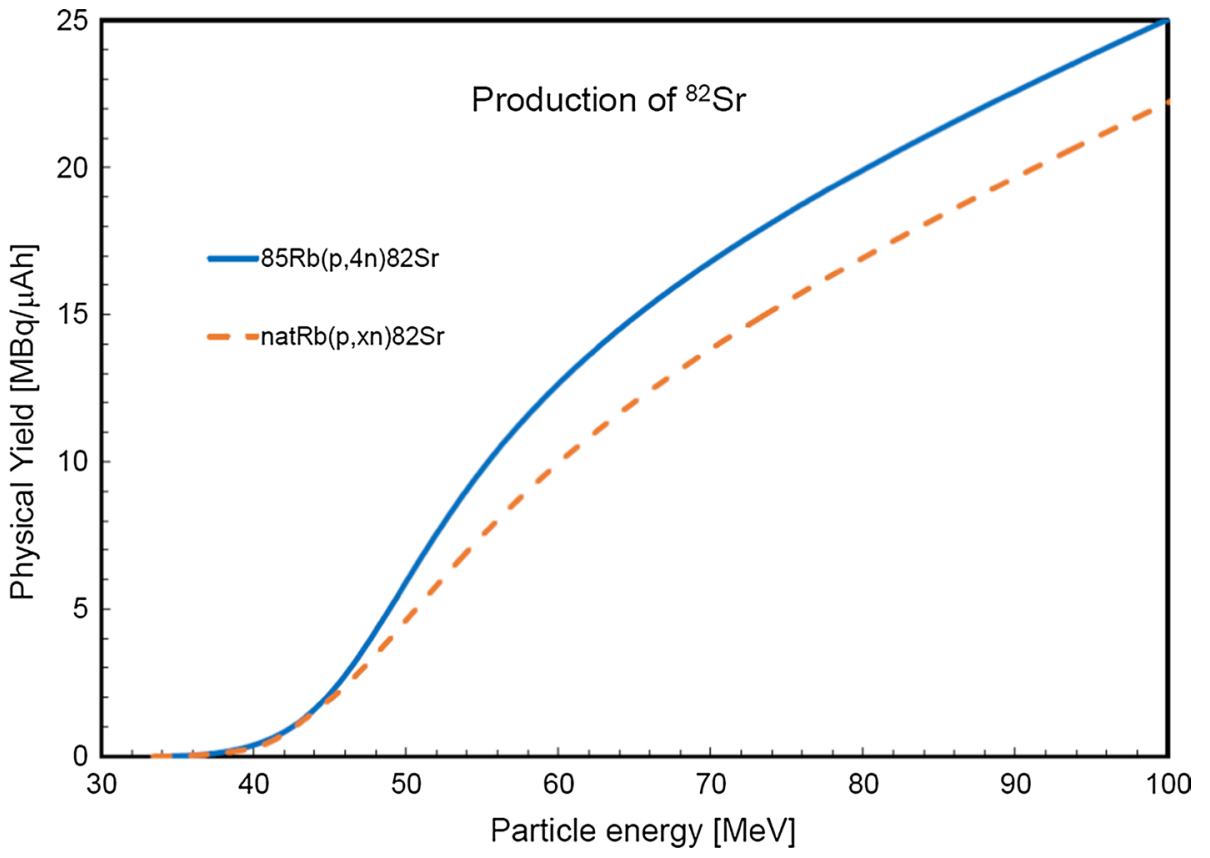




\section{Production of ${ }^{82 \mathrm{~m}} \mathrm{Rb}\left(\mathrm{T}_{1 / 2}=6.472 \mathrm{~h}\right)$}

Applications: Longer-lived ${ }^{82 \mathrm{~m}} \mathrm{Rb}$ isomeric state could possibly act as a substitute for generator-produced ${ }^{82} \mathrm{Rb}$ in PET cardiology centres that operate a cyclotron. However, this isomer suffers from a relatively high radiation burden that arises from the longer half-life and gamma-ray emissions. ${ }^{82 m} \mathrm{Rb}(6.472 \mathrm{~h}): \beta^{+}(21.2 \%)$, and $E_{\gamma}(\mathrm{keV})\left(P_{\gamma}(\%)\right): 554.35$ (62.4), 619.11 (37.98), 698.37 (26.3), 776.52 (84.39), 827.83 (21.0), 1044.08 (32.07), 1317.43 (23.7), 1474.88 (15.5). Evaluations have been undertaken of the ${ }^{82} \mathrm{Kr}(\mathrm{p}, \mathrm{n}){ }^{82 \mathrm{~m}} \mathrm{Rb}$ and ${ }^{82} \mathrm{Kr}(\mathrm{d}, 2 \mathrm{n}){ }^{82 \mathrm{~m}} \mathrm{Rb}$ reactions.
${ }^{82} \mathrm{Kr}(\mathrm{p}, \mathrm{n}){ }^{82 \mathrm{~m}} \mathrm{Rb}$

The four experimental datasets available in the literature are shown in Fig. 98 [231, 232] (each reference contains two datasets labelled (a) and (b)), together with the TENDL calculations. All datasets were used in the statistical fitting procedure. The data and their experimental uncertainties are shown in Fig. 99 together with the Padé fit $(L=9, N=33$, $\chi^{2}=1.13$ ) and estimated uncertainty in percentages, including $4 \%$ systematic uncertainty (right-hand scale).
Fig. 98 Four experimental datasets for the ${ }^{82} \mathrm{Kr}(\mathrm{p}, \mathrm{n})^{82 \mathrm{~m}} \mathrm{Rb}$ reaction available in the literature [231, 232] (each reference contains two datasets labelled (a) and (b)), and TENDL calculations

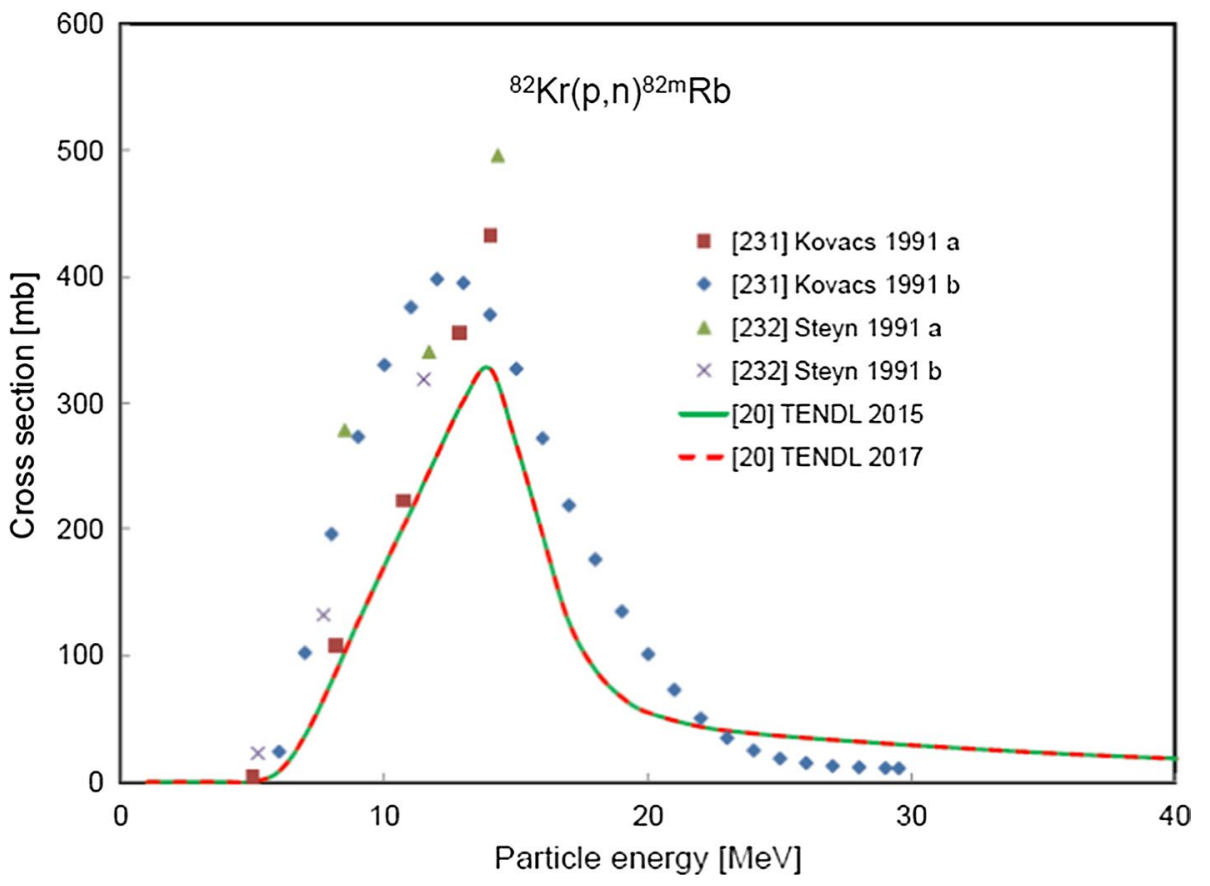


Fig. 99 Four experimental datasets for the ${ }^{82} \mathrm{Kr}(\mathrm{p}, \mathrm{n})^{82 \mathrm{~m}} \mathrm{Rb}$ reaction $[231,232]$ with the Padé fit $\left(L=9, N=33, \chi^{2}=1.13\right.$, solid line) and estimated total uncertainties in percentages, including $4 \%$ systematic uncertainty (dashed line, right-hand scale)

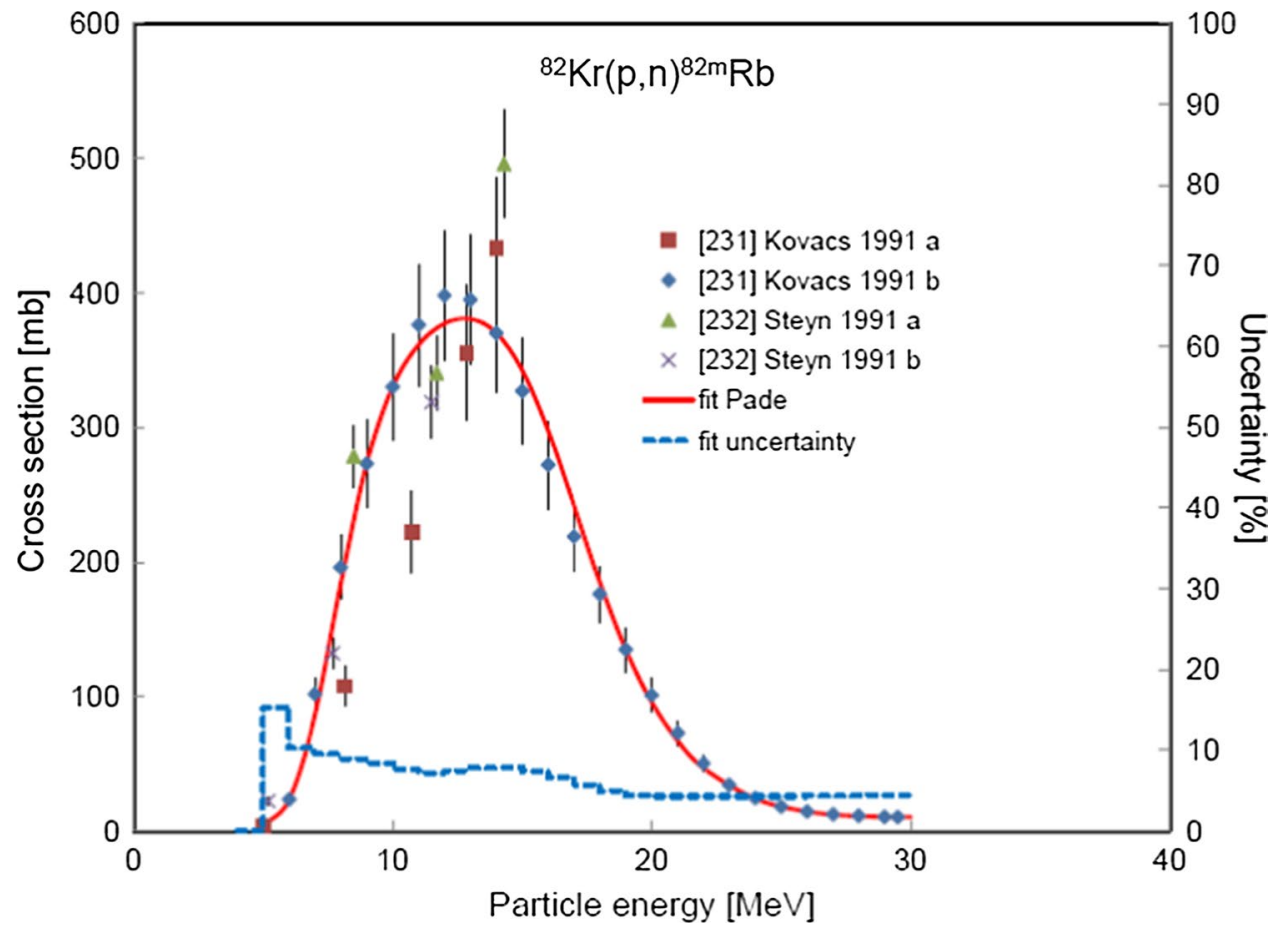




\section{${ }^{82} \operatorname{Kr}(d, 2 n){ }^{82 m} \mathrm{Rb}$}

A single experimental dataset available in the literature is shown in Fig. 100 [233] together with the TENDL calculations. This one dataset was used in the statistical fitting procedure. The data and their experimental uncertainties are shown in Fig. 101 together with the Padé fit $(L=5, N=14$, $\chi^{2}=2.27$ ) and estimated uncertainty in percentages, including $4 \%$ systematic uncertainty (right-hand scale).
Fig. 100 One experimental dataset for the ${ }^{82} \operatorname{Kr}(d, 2 n){ }^{82 m} R b$ reaction available in the literature [233], and TENDL calculations

Fig. 101 One experimental dataset for the ${ }^{82} \mathrm{Kr}(\mathrm{d}, 2 \mathrm{n})^{82 \mathrm{~m}} \mathrm{Rb}$ reaction [233] with the Padé fit $\left(L=5, N=14, \chi^{2}=2.27\right.$, solid line) and estimated total uncertainties in percentages, including $4 \%$ systematic uncertainty (dashed line, right-hand scale)
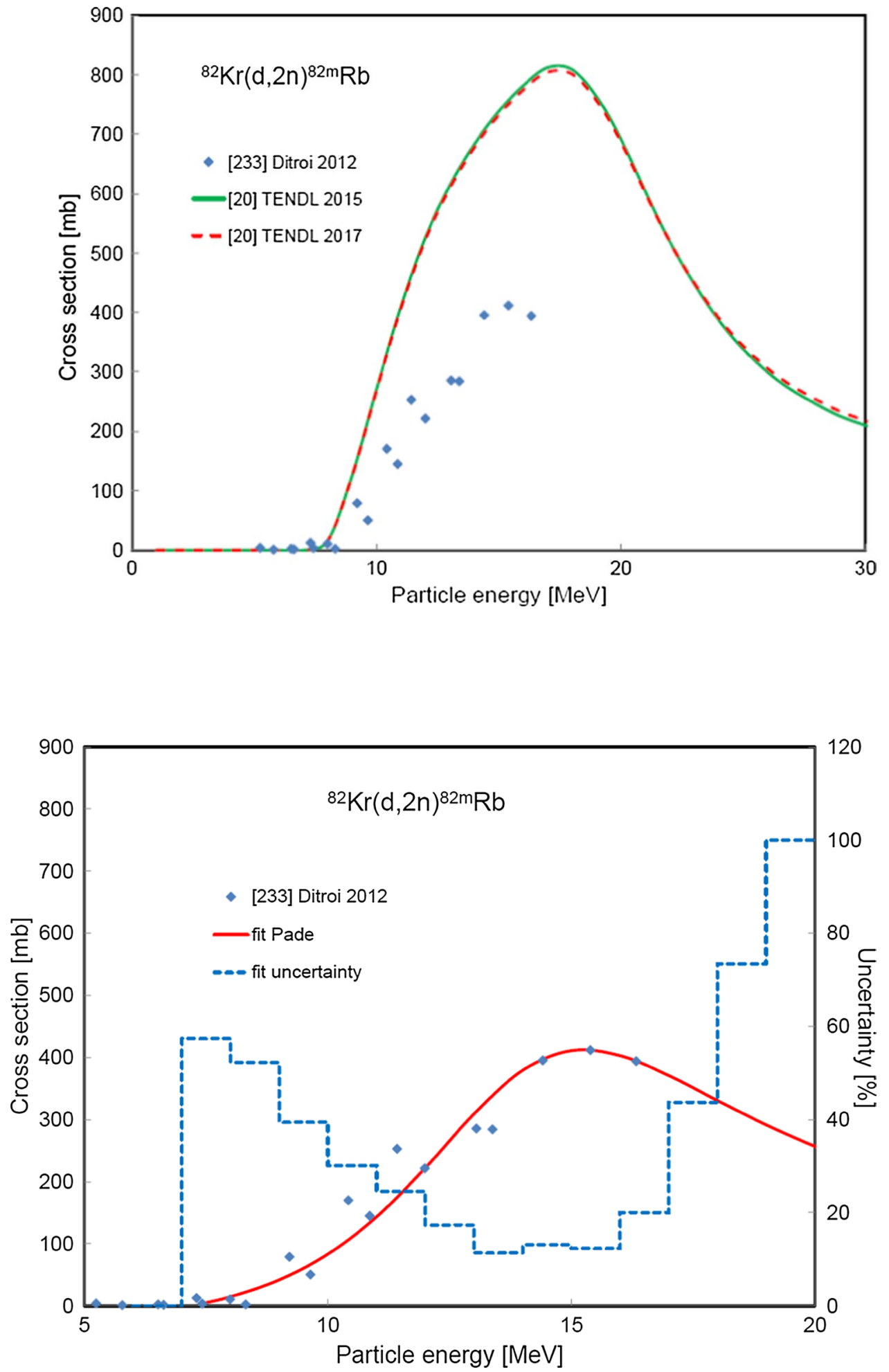
Thick target yields for production of ${ }^{82 \mathrm{~m}} \mathrm{Rb}$

See Fig. 102.

Fig. 102 Thick target yields calculated from the recommended cross sections for the ${ }^{82} \mathrm{Kr}(\mathrm{p}, \mathrm{n})^{82 \mathrm{~m}} \mathrm{Rb}$ and

${ }^{82} \mathrm{Kr}(\mathrm{d}, 2 \mathrm{n}){ }^{82 \mathrm{~m}} \mathrm{Rb}$ reactions

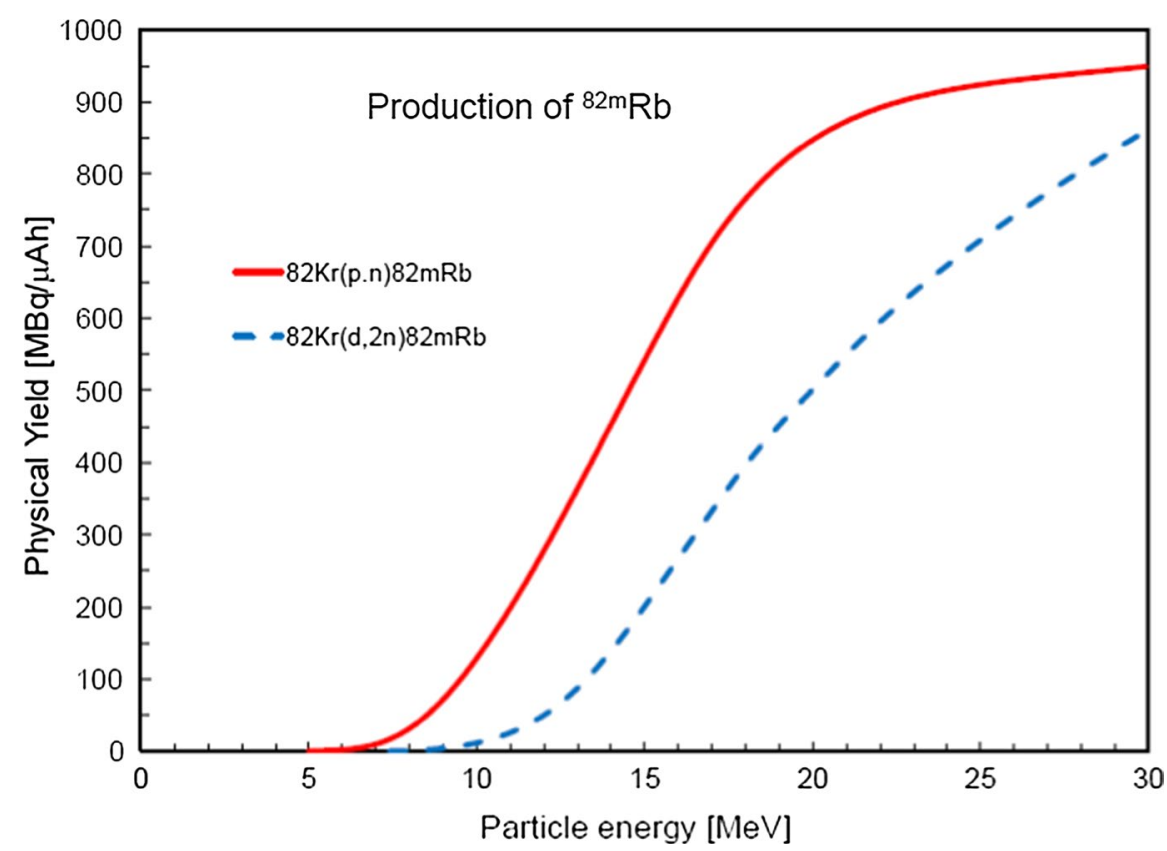




\section{Production of ${ }^{86} \mathrm{Y}\left(T_{1 / 2}=14.74 \mathrm{~h}\right)$}

Applications: Extensive studies of ${ }^{86} \mathrm{Y}$ have been performed as a positron emitter (31.9\%) with $14.74 \mathrm{~h}$ half-life that can adopted as a theranostic pair with clinically-established therapeutic beta-emitting ${ }^{90} \mathrm{Y}$. The role of ${ }^{86} \mathrm{Y}$ is to monitor the localised therapeutic dose distribution in the body for dosimetry calculations. Has also been studied for prostate cancer imaging, and used to label monoclonal antibodies in EGFR targeting. However, interest in this radionuclide has declined because of the high radiation burden and resultant poor imaging properties.

${ }^{86} Y(14.74 h): \beta^{+}(31.9 \%)$, and $E_{\gamma}(\mathrm{keV})\left(P_{\gamma}(\%)\right): 627.72$ (32.6), 1076.63 (82.5), 1153.05 (30.5).
Evaluations have been made of the ${ }^{86} \mathrm{Sr}(\mathrm{p}, \mathrm{n}){ }^{86} \mathrm{Y}$, ${ }^{88} \mathrm{Sr}(\mathrm{p}, 3 \mathrm{n}){ }^{86} \mathrm{Y}$ and ${ }^{85} \mathrm{Rb}(\alpha, 3 \mathrm{n}){ }^{86} \mathrm{Y}$ production routes.

\section{${ }^{86} \operatorname{Sr}(p, n){ }^{86} Y$}

The four experimental datasets available in the literature are shown in Fig. 103 [36, 82, 234, 235] together with the TENDL calculations. One dataset was rejected (Rösch et al. [235] (scattered data, and values too high near maximum)), while the three remaining sets were used in the statistical fitting procedure. The selected data and their experimental uncertainties are shown in Fig. 104 together with the Pade fit $(L=9, N=28$, $\left.\chi^{2}=0.615\right)$ and estimated uncertainty in percentages, including $4 \%$ systematic uncertainty (right-hand scale).
Fig. 103 Four experimental datasets for the ${ }^{86} \mathrm{Sr}(\mathrm{p}, \mathrm{n}){ }^{86} \mathrm{Y}$ reaction available in the literature [36, 82, 234, 235], and TENDL calculations

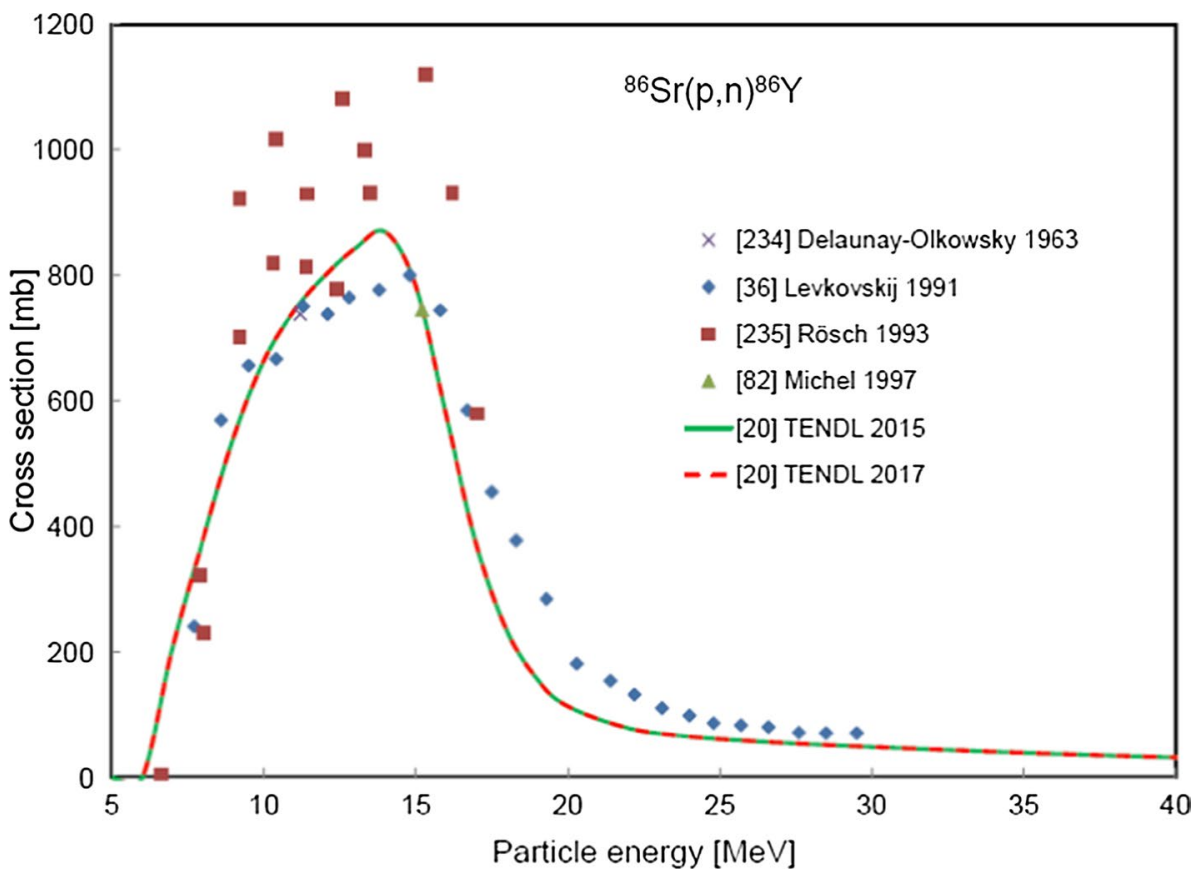


Fig. 104 Three selected experimental datasets for the

${ }^{86} \mathrm{Sr}(\mathrm{p}, \mathrm{n}){ }^{86} \mathrm{Y}$ reaction $[36,82$, 234] with the Padé fit ( $L=9$, $N=28, \chi^{2}=0.615$, solid line) and estimated total uncertainties in percentages, including $4 \%$ systematic uncertainty (dashed line, right-hand scale)

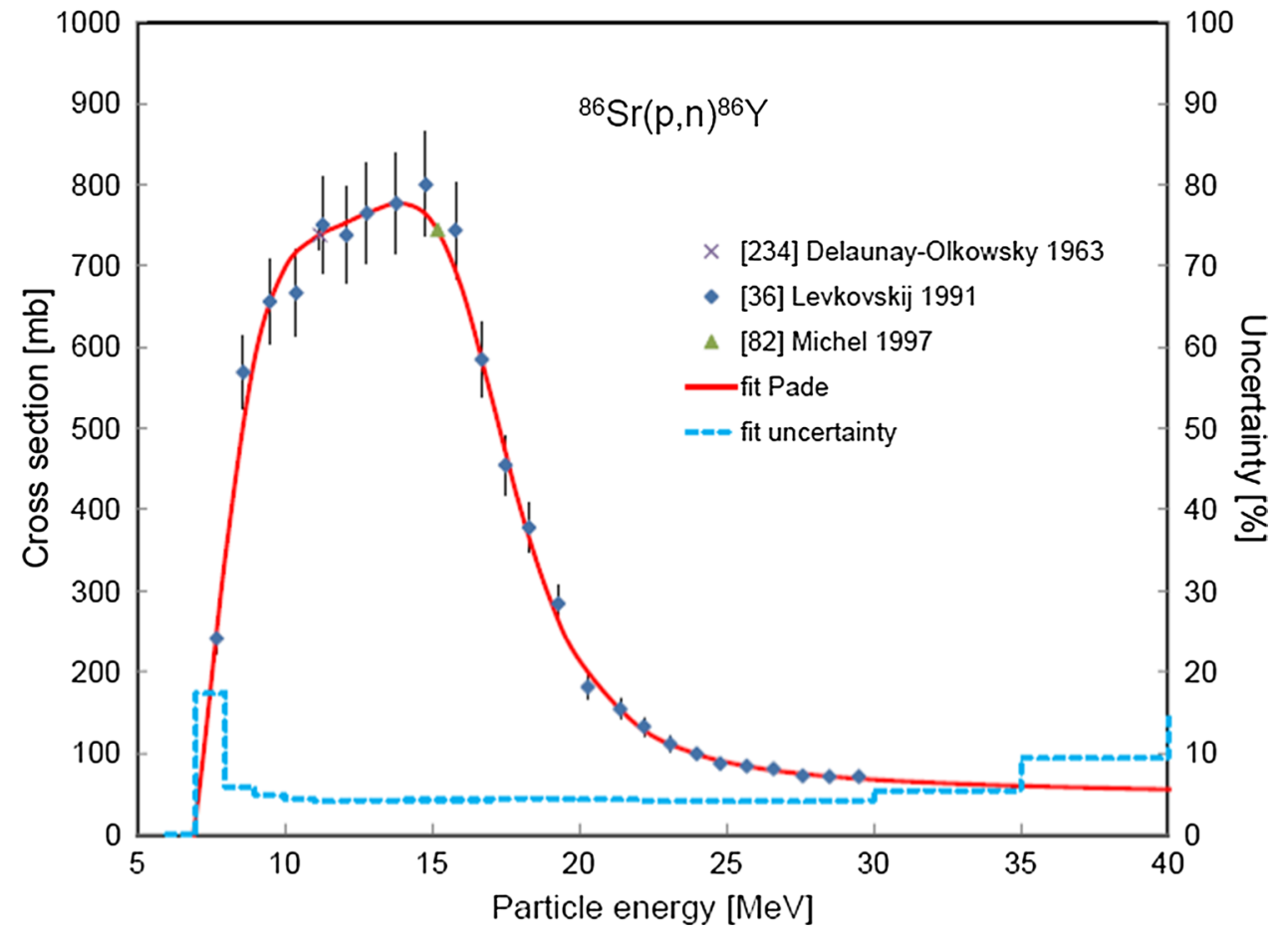




\section{${ }^{88} \operatorname{Sr}(p, 3 n){ }^{86} Y$}

The two experimental datasets available in the literature are shown in Fig. 105 [36, 236] together with the TENDL calculations. Both datasets were used in the statistical fitting procedure. These data and their experimental uncertainties are shown in Fig. 106 together with the Padé fit $(L=8$, $N=15, \chi^{2}=1.27$ ) and estimated uncertainty in percentages, including $4 \%$ systematic uncertainty (right-hand scale).
Fig. 105 Two experimental datasets for the ${ }^{88} \operatorname{Sr}(\mathrm{p}, 3 \mathrm{n}){ }^{86} \mathrm{Y}$ reaction available in the literature [36, 236], and TENDL calculations
Fig. 106 Two experimental datasets for the ${ }^{88} \mathrm{Sr}(\mathrm{p}, 3 \mathrm{n}){ }^{86} \mathrm{Y}$ reaction $[36,236]$ with the Padé fit $\left(L=8, N=15, \chi^{2}=1.27\right.$, solid line) and estimated total uncertainties in percentages, including $4 \%$ systematic uncertainty (dashed line, right-hand scale)
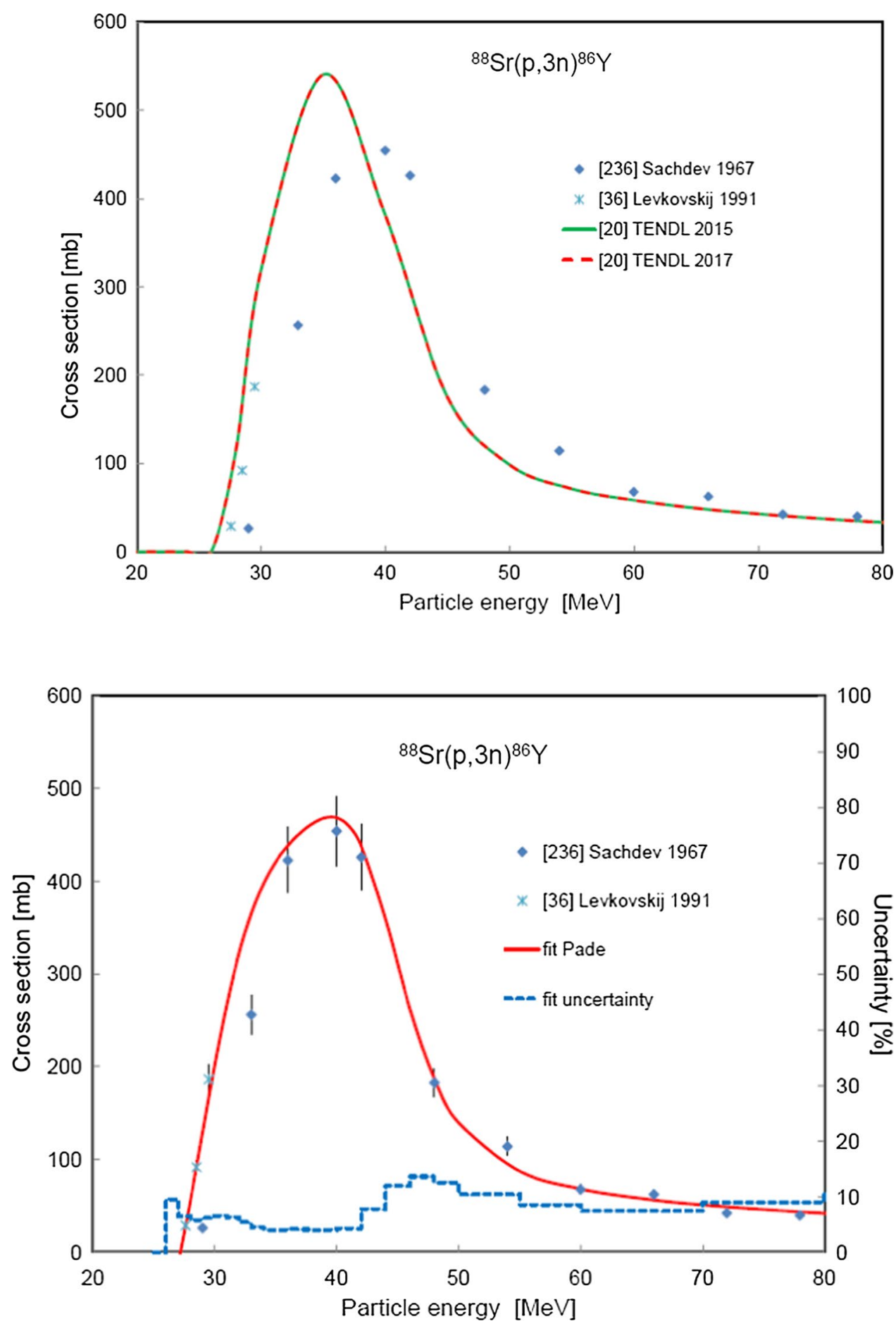
${ }^{85} \mathrm{Rb}(a, 3 n){ }^{86} \mathrm{Y}$

Five experimental datasets available in the literature are shown in Fig. 107 [36, 237-240] together with the TENDL calculations. Three datasets were rejected (Guin et al. [239], Iwata [237], and Agarwal et al. [240] (values refer to direct ground state production only, and are not cumulative). The data points of Demeyer et al. [238] below $45 \mathrm{MeV}$ are discrepant, and were also deleted. Thus, the remaining data points for only two datasets were used in the statistical fitting procedure $[36,238]$. These selected data and their experimental uncertainties are shown in Fig. 108 together with the Padé fit ( $\left.L=8, N=32, \chi^{2}=0.91\right)$ and estimated uncertainty in percentages, including $4 \%$ systematic uncertainty (righthand scale).
Fig. 107 Five experimental datasets for the ${ }^{85} \mathrm{Rb}(\alpha, 3 \mathrm{n})^{86} \mathrm{Y}$ reaction available in the literature [36, 237-240], and TENDL calculations

Fig. 108 Two selected experimental datasets for the ${ }^{85} \mathrm{Rb}(\alpha, 3 \mathrm{n}){ }^{86} \mathrm{Y}$ reaction [36, 238 ] with the Padé fit $(L=8$, $N=32, \chi^{2}=0.91$, solid line) and estimated total uncertainties in percentages, including $4 \%$ systematic uncertainty (dashed line, right-hand scale)
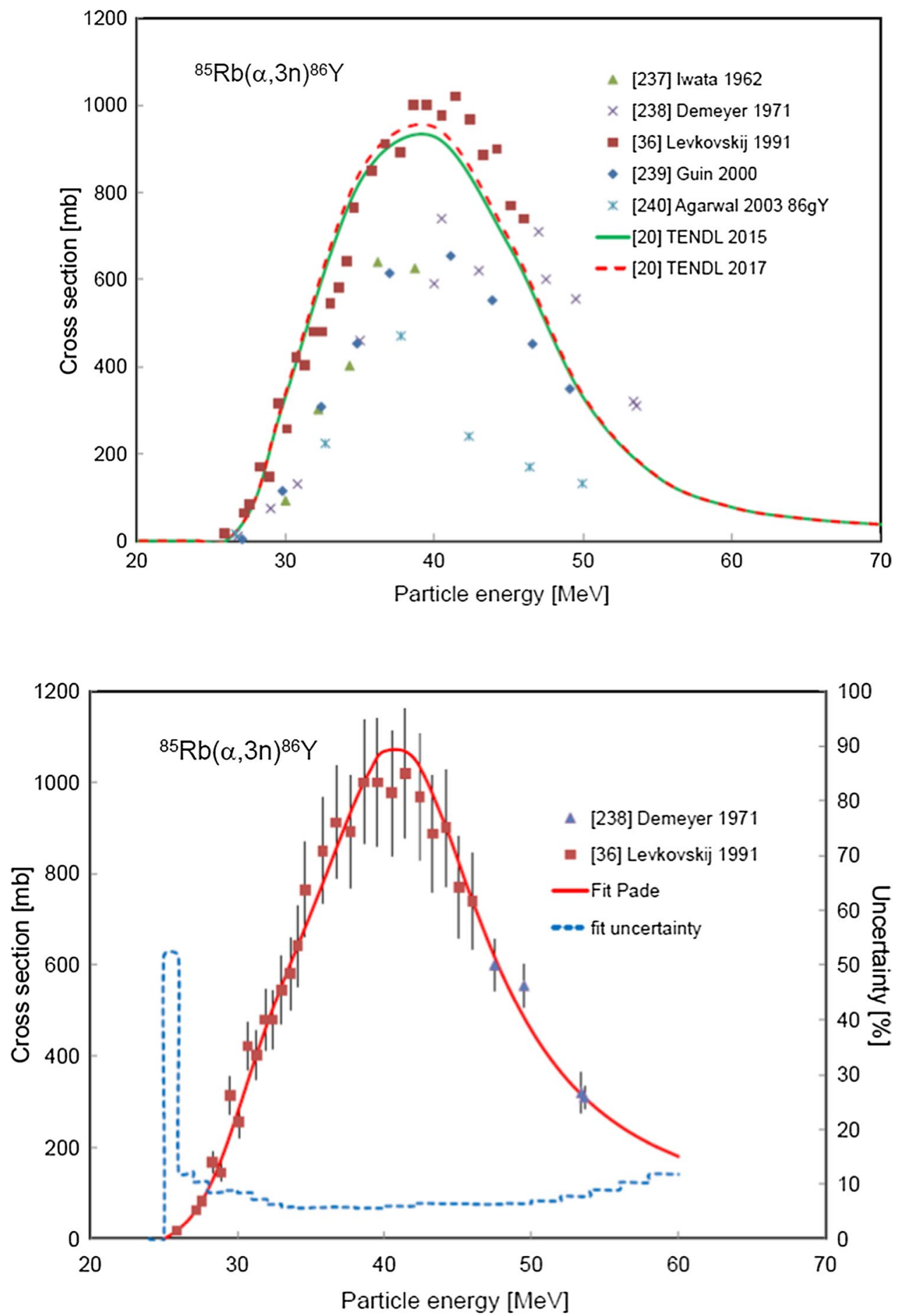
Thick target yields for production of ${ }^{86} \mathrm{Y}$

See Fig. 109.

Fig. 109 Thick target yields calculated from the recommended cross sections for the

${ }^{86} \mathrm{Sr}(\mathrm{p}, \mathrm{n}){ }^{86} \mathrm{Y},{ }^{88} \mathrm{Sr}(\mathrm{p}, 3 \mathrm{n}){ }^{86} \mathrm{Y}$ and

${ }^{85} \mathrm{Rb}(\alpha, 3 \mathrm{n}){ }^{86} \mathrm{Y}$ reactions

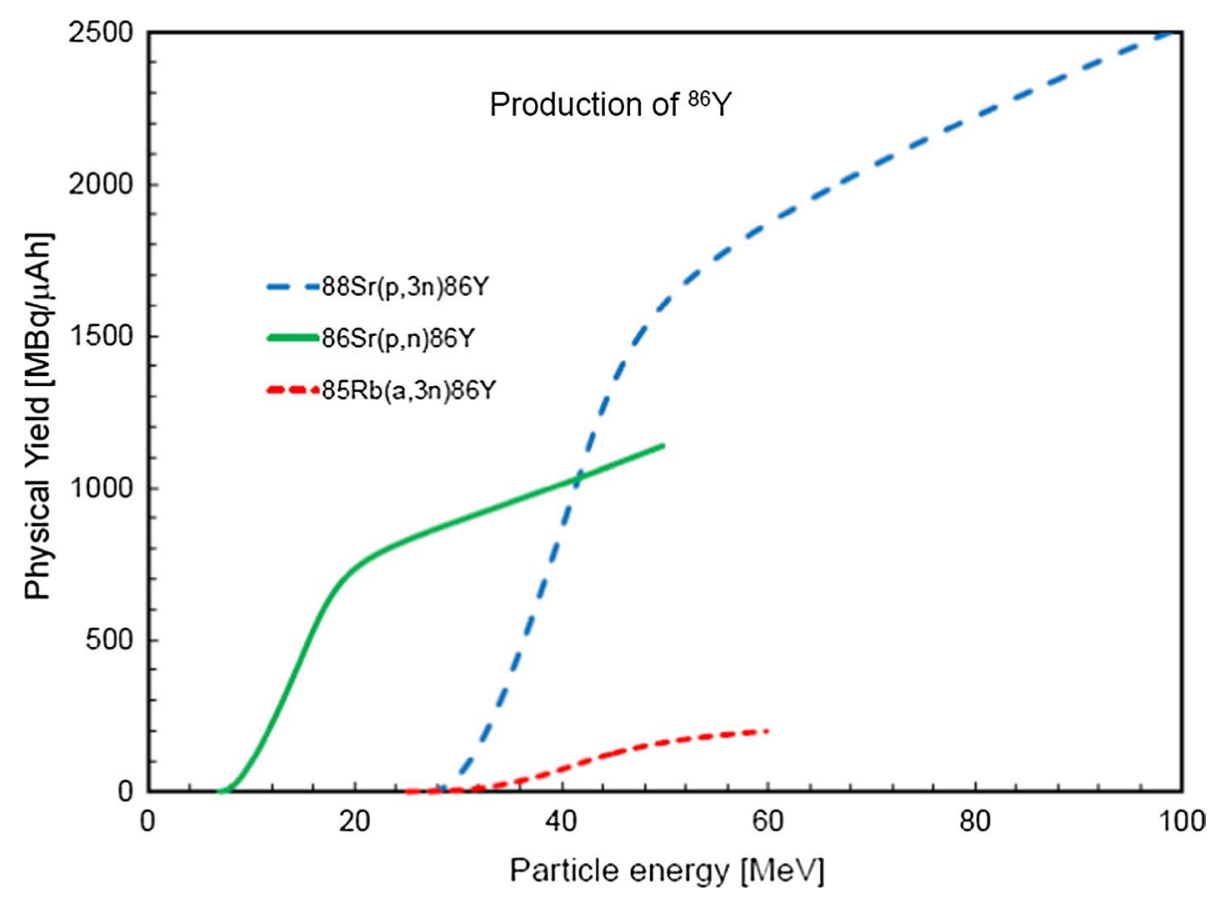




\section{Production of ${ }^{89} \mathrm{Zr}\left(T_{1 / 2}=78.41 \mathrm{~h}\right)$}

Applications: Long-lived positron-emitting ${ }^{89} \mathrm{Zr}$ has been extensively studied with respect to following the in vivo behaviour of therapeutic monoclonal antibodies (mAbs) and other biomolecules with slow biokinetics. One significant disadvantage is the limited number of suitable ${ }^{89} \mathrm{Zr}$ chelating agents and difficulties related to their development.

${ }^{89} \mathrm{Zr}(78.41 \mathrm{~h}): \beta^{+}(22.74 \%)$, and $E_{\gamma}(\mathrm{keV})\left(P_{\gamma}(\%)\right): 909.15$ (99.04).

Evaluations have been made of the ${ }^{89} \mathrm{Y}(\mathrm{p}, \mathrm{n}){ }^{89} \mathrm{Zr}$ and ${ }^{89} \mathrm{Y}(\mathrm{d}, 2 \mathrm{n}){ }^{89} \mathrm{Zr}$ production routes.
${ }^{89} \mathrm{Y}(\mathrm{p}, \mathrm{n}){ }^{89} \mathrm{Zr}$

The sixteen experimental datasets available in the literature are shown in Fig. 110 [36, 56, 82, 110, 234, 241-251] together with the TENDL calculations. Five datasets were rejected (Birattari et al. [244] (energy shift), Blosser and Handley [56] (value too high), Satheesh et al. [250] (energy shift), Delaunay-Olkowsky et al. [234] (value too low), and Saha et al. [242] (values too high)), and the remaining eleven datasets were used in the statistical fitting procedure. The selected data and their experimental uncertainties are shown in Fig. 111 together with the Padé fit $(L=11, N=316$, $\left.\chi^{2}=3.74\right)$ and estimated uncertainty in percentages, including $4 \%$ systematic uncertainty (right-hand scale).
Fig. 110 Sixteen experimental datasets for the ${ }^{89} \mathrm{Y}(\mathrm{p}, \mathrm{n})^{89} \mathrm{Zr}$ reaction available in the literature $[36,56,82,110,234$, 241-251], and TENDL calculations

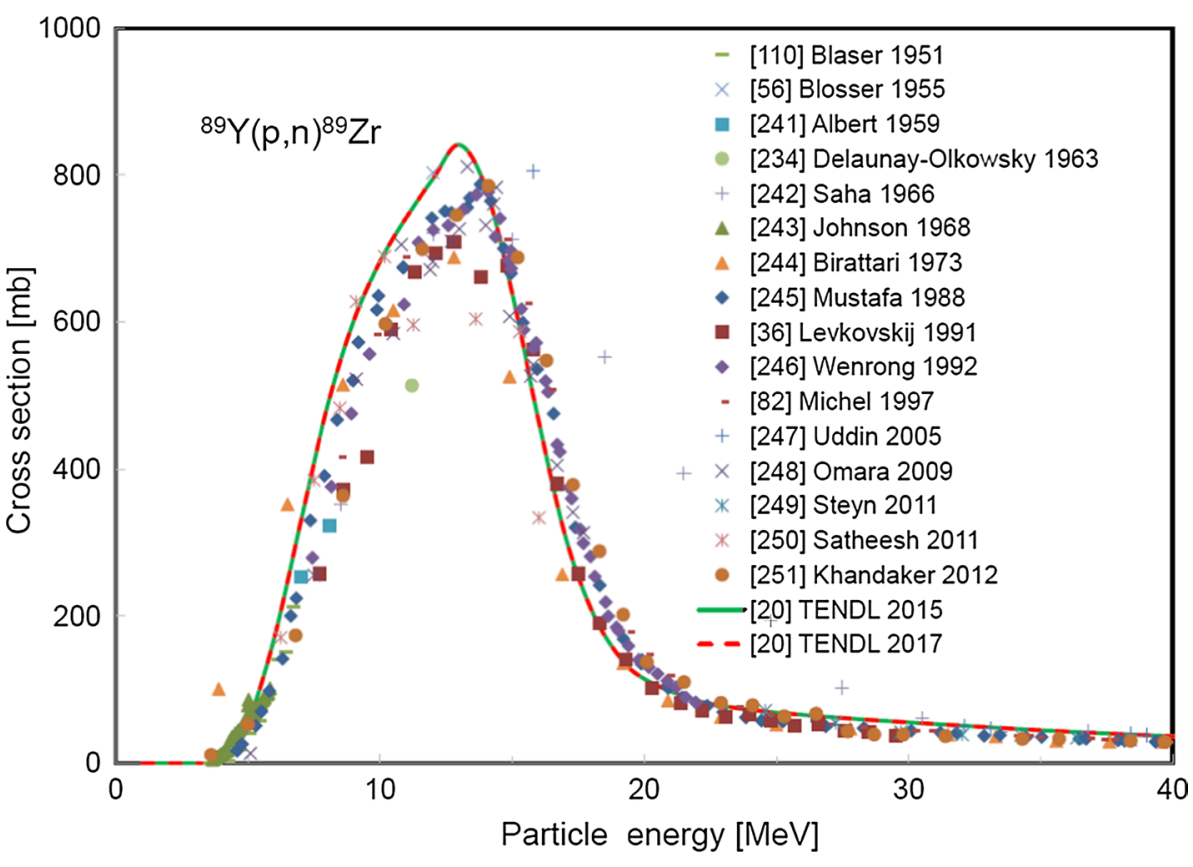


Fig. 111 Eleven selected experimental datasets for the ${ }^{89} \mathrm{Y}(\mathrm{p}, \mathrm{n}){ }^{89} \mathrm{Zr}$ reaction $[36$, $82,110,241,243,245-249$, 251] with the Padé fit $(L=11$, $N=316, \chi^{2}=3.74$, solid line) and estimated total uncertainties in percentages, including $4 \%$ systematic uncertainty (dashed line, right-hand scale)

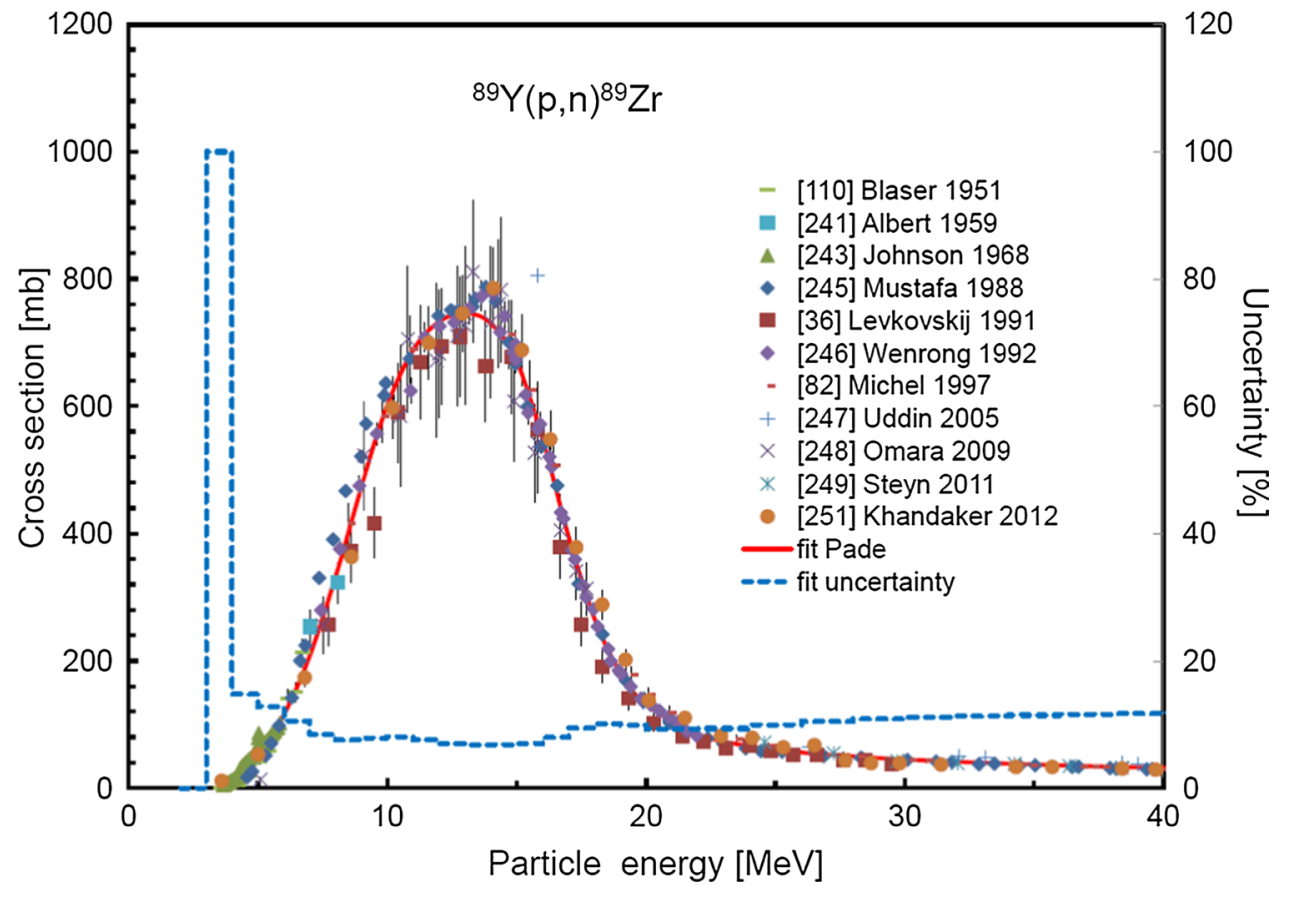


${ }^{89} \mathrm{Y}(\mathrm{d}, 2 \mathrm{n}){ }^{89} \mathrm{Zr}$

The seven experimental datasets available in the literature are shown in Fig. 112 [252-258] together with the TENDL calculations. Two datasets were rejected (La Gamma and Nassiff [253] (values too low), and Degering et al. [255] (energy shift)), and the remaining five sets were used in the statistical fitting procedure. The selected data and their experimental uncertainties are shown in Fig. 113 together with the Padé fit $\left(L=9, N=64, \chi^{2}=2.95\right)$ and estimated uncertainty in percentages, including $4 \%$ systematic uncertainty (right-hand scale).
Fig. 112 Seven experimental datasets for the ${ }^{89} \mathrm{Y}(\mathrm{d}, 2 \mathrm{n})^{89} \mathrm{Zr}$ reaction available in the literature [252-258], and TENDL calculations

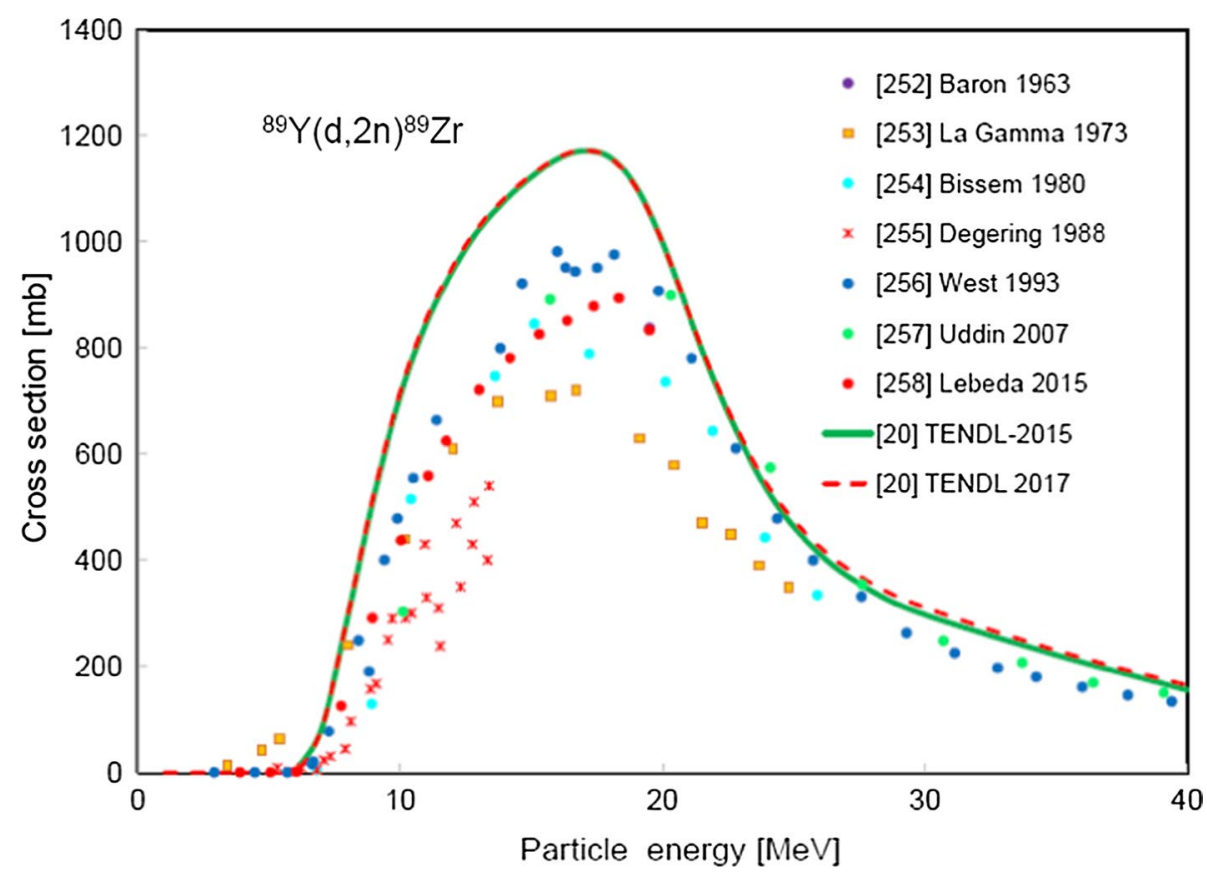

Fig. 113 Five selected experimental datasets for the ${ }^{89} \mathrm{Y}(\mathrm{d}, 2 \mathrm{n}){ }^{89} \mathrm{Zr}$ reaction $[252$, 254, 256-258] with the Padé fit $\left(L=9, N=64, \chi^{2}=2.95\right.$, solid line) and estimated total uncertainties in percentages, including $4 \%$ systematic uncertainty (dashed line, right-hand scale)

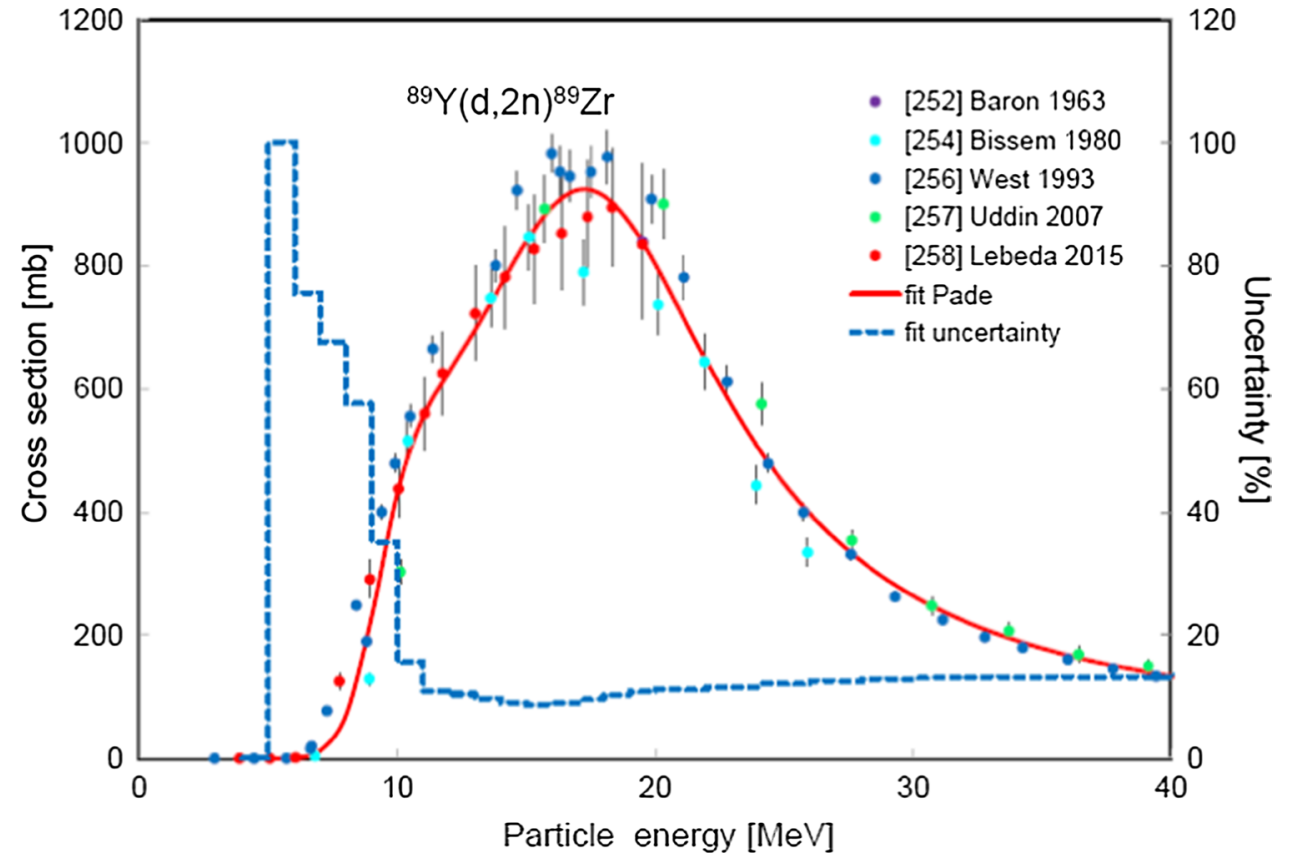


Thick target yields for production of ${ }^{89} \mathrm{Zr}$

See Fig. 114.

Fig. 114 Thick target yields calculated from the recommended cross sections for the ${ }^{89} \mathrm{Y}(\mathrm{p}, \mathrm{n}){ }^{89} \mathrm{Zr}$ and ${ }^{89} \mathrm{Y}(\mathrm{d}, 2 \mathrm{n}){ }^{89} \mathrm{Zr}$ reactions

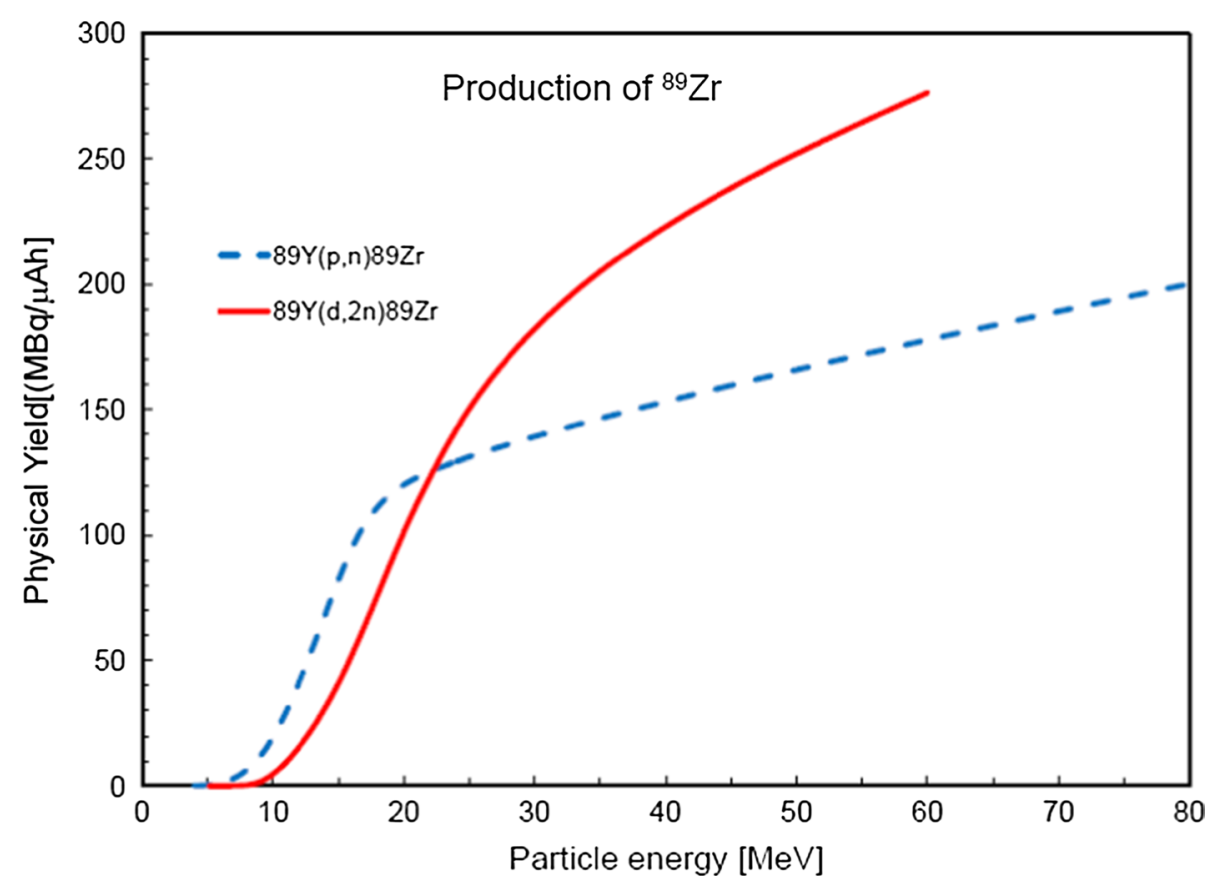




\section{Production of ${ }^{90} \mathrm{Nb}\left(T_{1 / 2}=14.60 \mathrm{~h}\right)$}

Applications: As a non-conventional positron emitter, ${ }^{90} \mathrm{Nb}$ with a half-life of $14.60 \mathrm{~h}$ can be used to visualise and quantify processes with medium and slow kinetics, such as tumour accumulation of antibodies and antibody fragments, or polymers and other nanoparticles. Exhibits promise in immuno-PET, although a search for appropriate chelators is desirable. Also emits several high-energy gamma rays that increase the radiation burden.

${ }^{90} \mathrm{Nb}(14.60 \mathrm{~h}): \beta^{+}(51.2 \%)$, and $E_{\gamma}(\mathrm{keV})\left(P_{\gamma}(\%)\right): 132.716$ (4.13), 141.178 (66.8), 1129.224 (92.7).
Evaluations have been undertaken of the ${ }^{93} \mathrm{Nb}(\mathrm{p}, \mathrm{x}){ }^{90} \mathrm{Nb}$ and ${ }^{89} \mathrm{Y}(\alpha, 3 \mathrm{n}){ }^{90} \mathrm{Nb}$ production.

\section{${ }^{93} \mathrm{Nb}(p, x){ }^{90} \mathrm{Nb}$}

The six experimental datasets available in the literature are shown in Fig. 115 [82, 249, 259-262] together with the TENDL calculations. All datasets were used in the statistical fitting procedure. The data and their experimental uncertainties are shown in Fig. 116 together with the Padé fit $(L=9$, $N=94, \chi^{2}=3.18$ ) and estimated uncertainty in percentages, including $4 \%$ systematic uncertainty (right-hand scale).
Fig. 115 Six experimental datasets for the ${ }^{93} \mathrm{Nb}(\mathrm{p}, \mathrm{x})^{90} \mathrm{Nb}$ reaction available in the literature [82, 249, 259-262], and

TENDL calculations

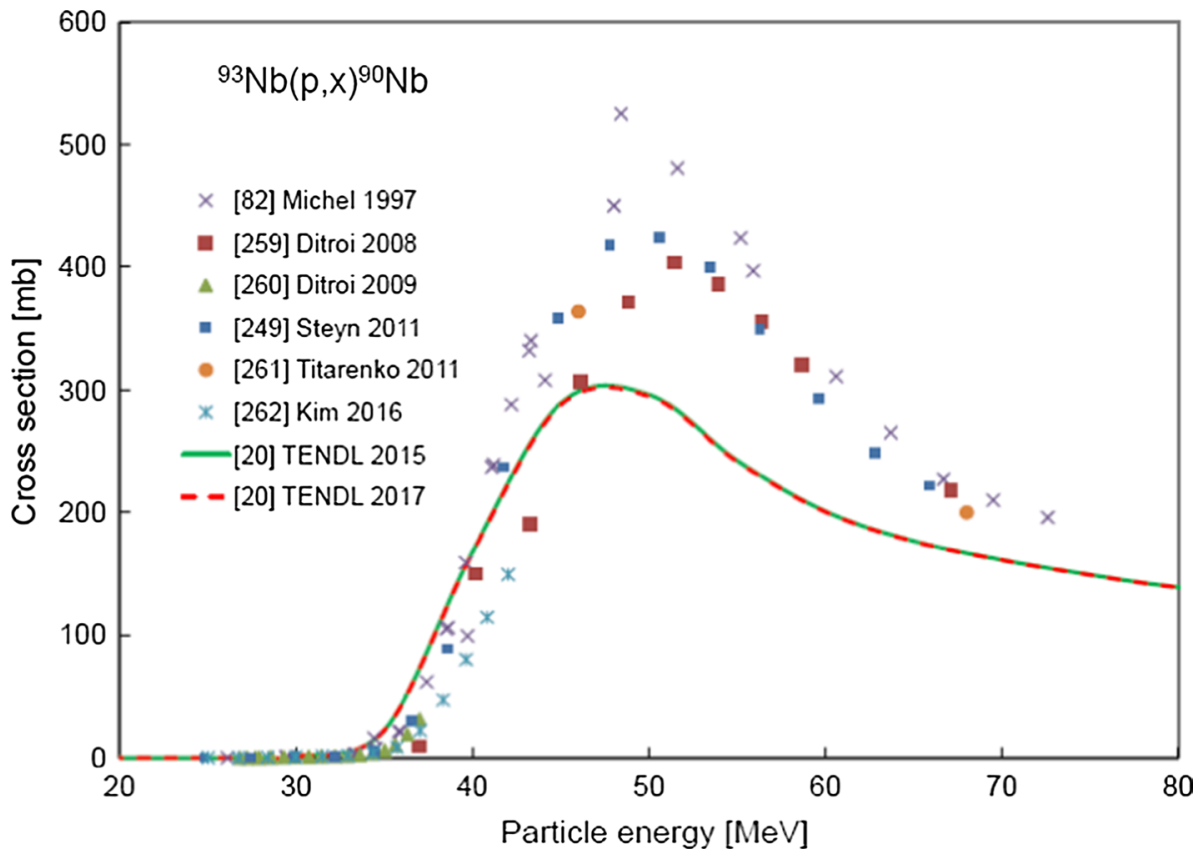


Fig. 116 Six experimental datasets for the ${ }^{93} \mathrm{Nb}(\mathrm{p}, \mathrm{x}){ }^{90} \mathrm{Nb}$ reaction [82, 249, 259-262] with the Padé fit $(L=9, N=94$, $\chi^{2}=3.18$, solid line) and estimated total uncertainties in percentages, including $4 \%$ systematic uncertainty (dashed line, right-hand scale)

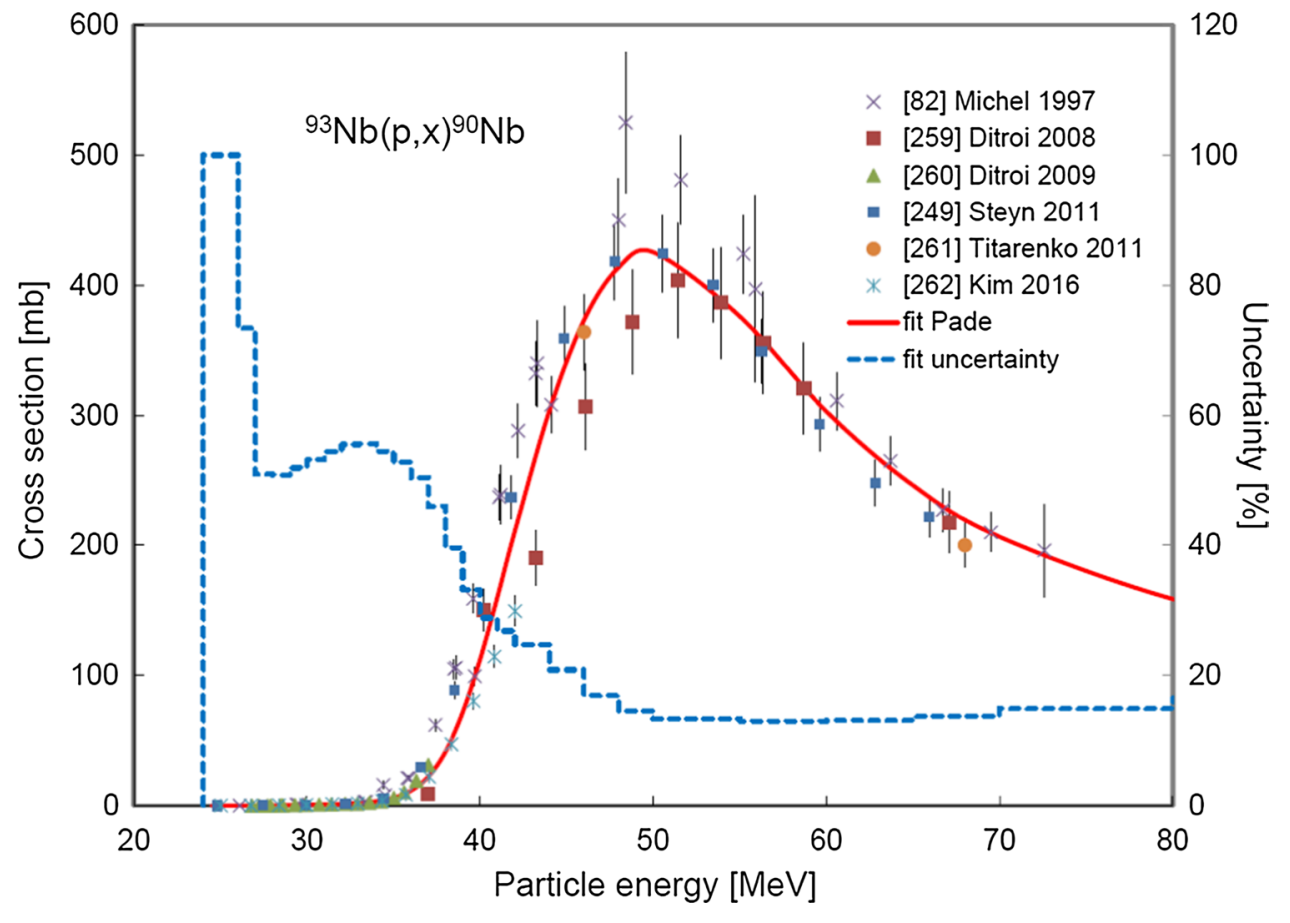


${ }^{89} \mathrm{Y}(a, 3 \mathrm{n}){ }^{90} \mathrm{Nb}$

The six experimental datasets available in the literature are shown in Fig. 117 [36, 263-267] together with the TENDL calculations. Four datasets were rejected (Singh et al. [266], Chaubey and Rizvi [265], Mukherjee et al. [264], and
Smend et al. [263], all systematically lower values), while the remaining two sets were used in the statistical fitting procedure. The selected data and their experimental uncertainties are shown in Fig. 118 together with the Padé fit $(L=16$, $N=33, \chi^{2}=1.29$ ) and estimated uncertainty in percentages, including $4 \%$ systematic uncertainty (right-hand scale).
Fig. 117 Six experimental datasets for the ${ }^{89} \mathrm{Y}(\alpha, 3 \mathrm{n}){ }^{90} \mathrm{Nb}$ reaction available in the literature [36, 263-267], and TENDL calculations
Fig. 118 Two selected experimental datasets for the ${ }^{89} \mathrm{Y}(\alpha, 3 \mathrm{n}){ }^{90} \mathrm{Nb}$ reaction $[36$, 267] with the Padé fit ( $L=16$, $N=33, \chi^{2}=1.29$, solid line) and estimated total uncertainties in percentages, including $4 \%$ systematic uncertainty (dashed line, right-hand scale)
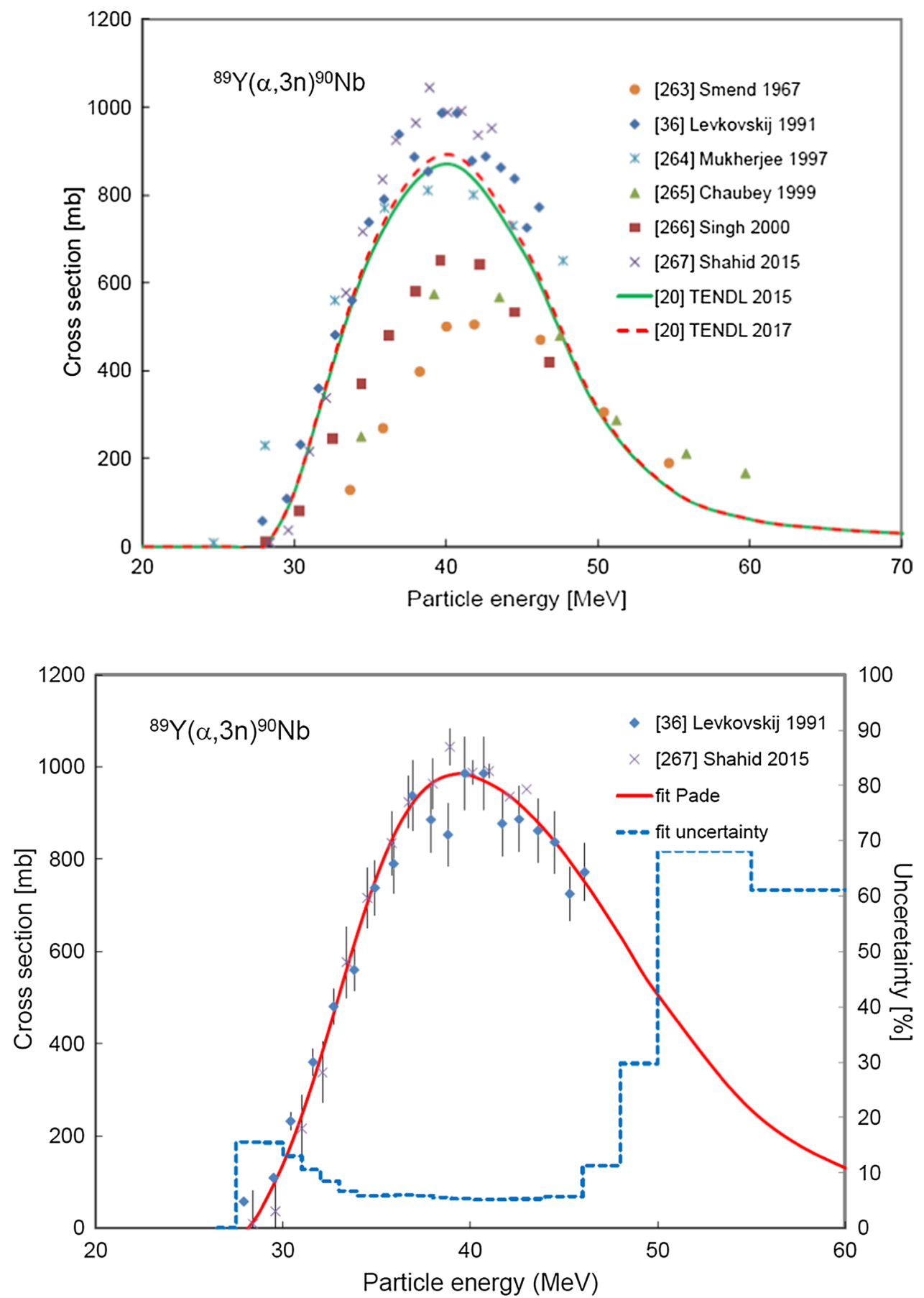


\section{Thick target yields for production of ${ }^{90} \mathrm{Nb}$}

See Fig. 119.

Fig. 119 Thick target yields calculated from the recommended cross sections for the ${ }^{93} \mathrm{Nb}(\mathrm{p}, \mathrm{x})^{90} \mathrm{Nb}$ and

${ }^{89} \mathrm{Y}(\alpha, 3 \mathrm{n}){ }^{90} \mathrm{Nb}$ reactions

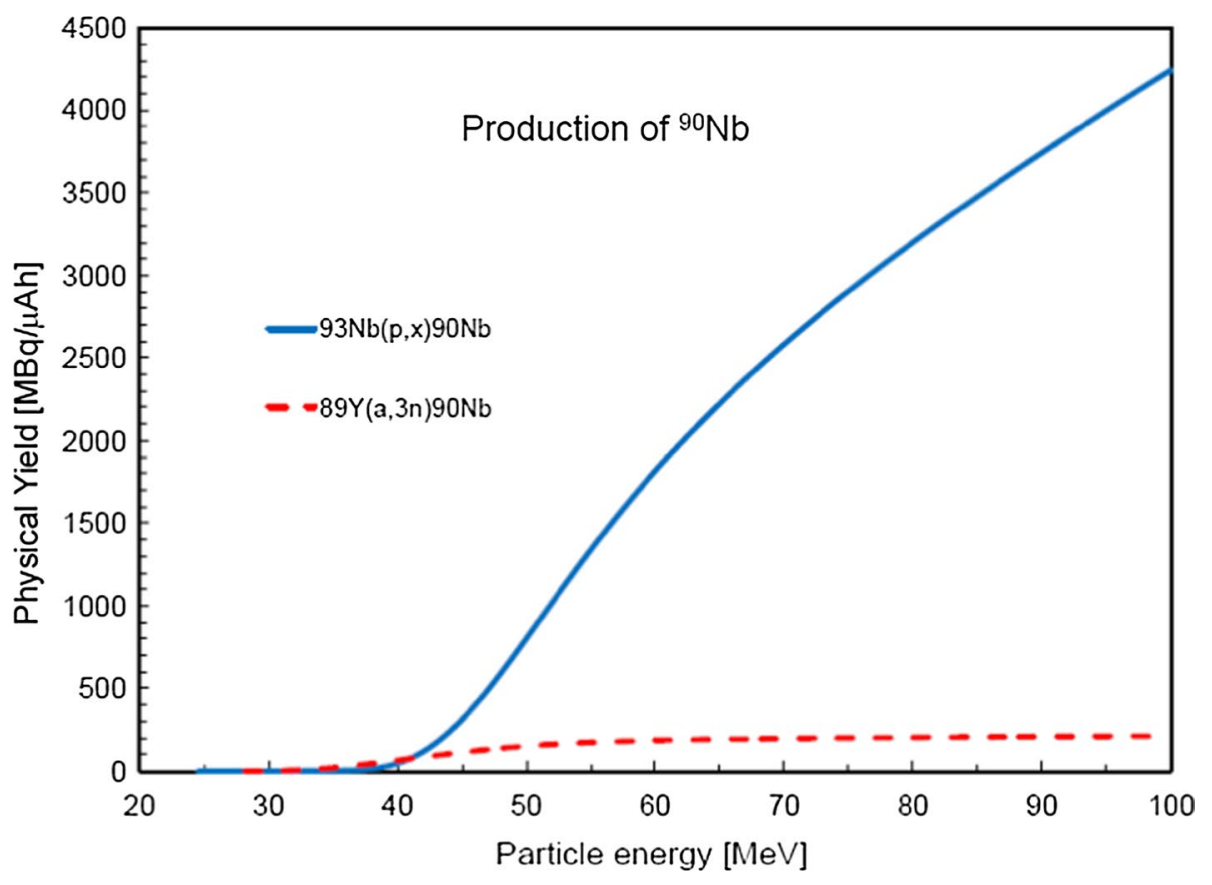




\section{Production of ${ }^{94 \mathrm{~m}} \mathrm{Tc}\left(T_{1 / 2}=52.0 \mathrm{~min}\right)$}

Applications: Gamma-ray emitting ${ }^{99 \mathrm{~m}} \mathrm{Tc}$ is the most widespread medical radionuclide for diagnosis, whereas ${ }^{94 \mathrm{~m}} \mathrm{Tc}$ with a half-life of $52.0 \mathrm{~min}$. is a positron emitter with a positron branch of $70.2 \%$ and $E_{\beta+}(\max )$ of $2.44 \mathrm{MeV}$. Therefore, there has been interest in ${ }^{94 \mathrm{~m}} \mathrm{Tc}$ as a PET analogue to ${ }^{99 \mathrm{~m}} \mathrm{Tc}$ since they both undergo the same chemistry. Obvious disadvantages of ${ }^{94 \mathrm{~m}} \mathrm{Tc}$ are the rather short half-life of $52.0 \mathrm{~min}$., with many accompanying gamma rays and the inability to prepare the pure isomer without also generating significant amounts of ground state ${ }^{94 \mathrm{~g}} \mathrm{Tc}$.

${ }^{94 m} T c(52.0 \mathrm{~min}): \beta^{+}(70.2 \%)$, and $E_{\gamma}(\mathrm{keV})\left(P_{\gamma}(\%)\right): 871.05$ (94.2), 1522.1 (4.5), 1868.68 (5.7).

Evaluations have been made of the ${ }^{92} \mathrm{Mo}(\alpha, \mathrm{x})^{94 \mathrm{~m}} \mathrm{Tc}$ and ${ }^{94} \mathrm{Mo}(\mathrm{p}, \mathrm{n}){ }^{94 \mathrm{~m}} \mathrm{Tc}$ production routes.
${ }^{92} \mathrm{Mo}(a, x){ }^{94 \mathrm{~m}} \mathrm{Tc}$

The four experimental datasets available in the literature are shown in Fig. 120 [36, 268-270] together with the TENDL calculations. Three datasets were rejected (Graf and Münzel [268], Denzler et al. [269], and Ditrói et al. [270], all contradictory sets of data), while the remaining single set of Levkovskij [36] was used in the statistical fitting procedure (and also accepted as a standard for the monitoring of $\alpha$ beams). The selected data and their experimental uncertainties are shown in Fig. 121 together with the Padé fit $(L=12, N=28$, $\chi^{2}=1.33$ ) and estimated uncertainty in percentages, including $4 \%$ systematic uncertainty (right-hand scale).
Fig. 120 Four experimental datasets for the ${ }^{92} \mathrm{Mo}(\alpha, \mathrm{x})^{94 \mathrm{~m}} \mathrm{Tc}$ reaction available in the literature [36, 268-270], and TENDL calculations

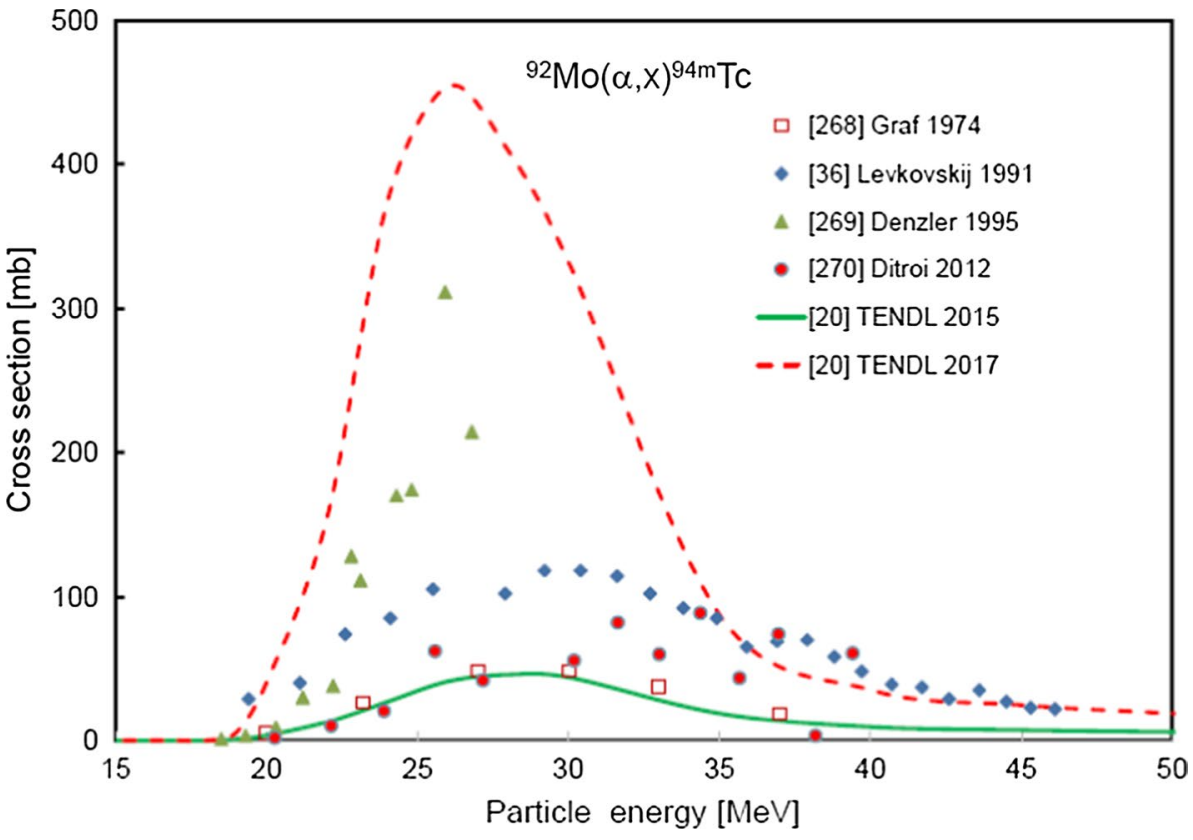


Fig. 121 One selected experimental dataset for the ${ }^{92} \mathrm{Mo}(\alpha, \mathrm{x})^{94 \mathrm{~m}} \mathrm{Tc}$ reaction [36] with the Padé fit $(L=12, N=28$, $\chi^{2}=1.33$, solid line) and estimated total uncertainties in percentages, including $4 \%$ systematic uncertainty (dashed line, right-hand scale)

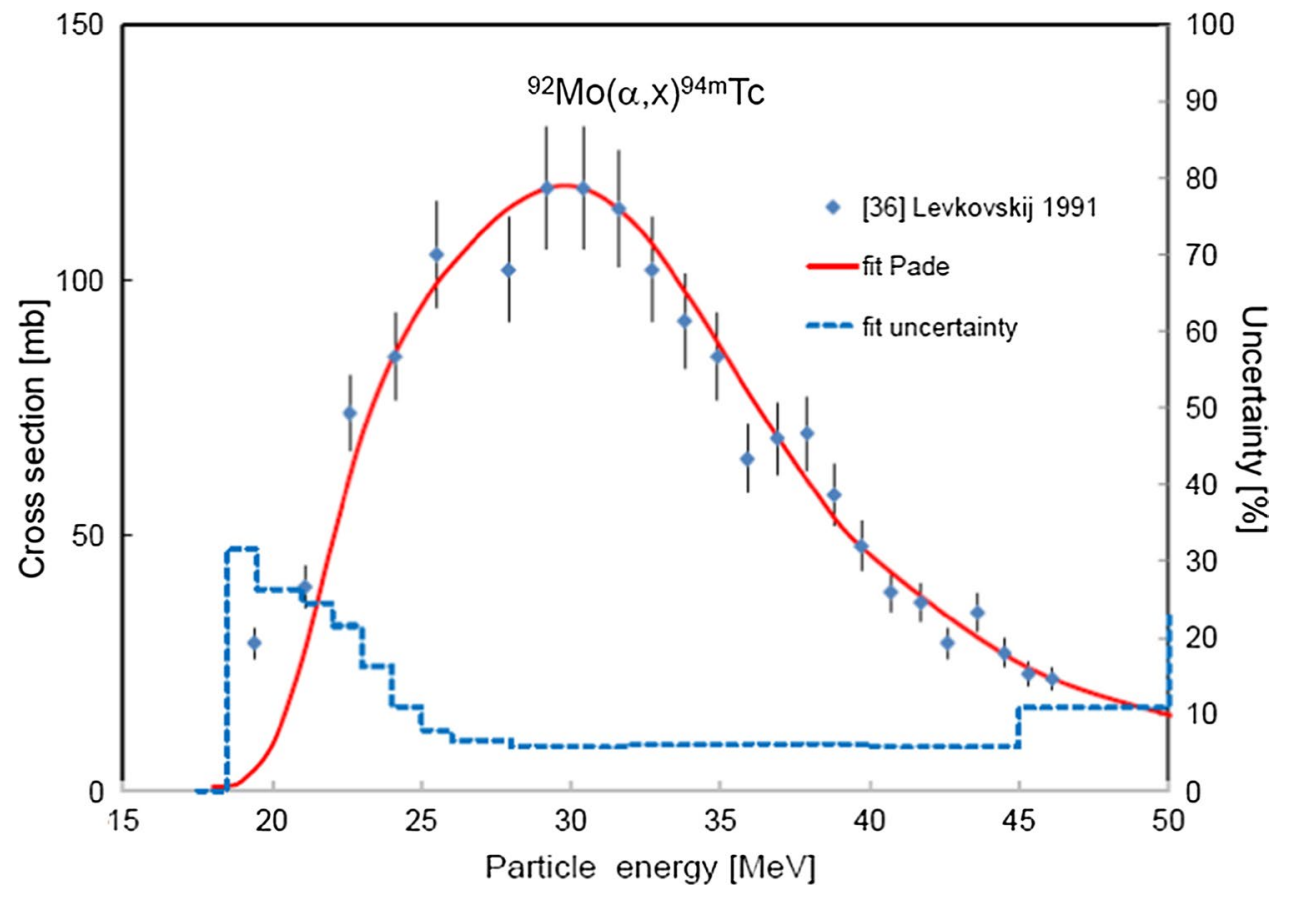


${ }^{94} \mathrm{Mo}(p, \mathrm{n}){ }^{94 \mathrm{~m}} \mathrm{Tc}$

The seven experimental datasets available in the literature are shown in Fig. 122 [36, 142, 271-275] together with the TENDL calculations. All datasets were used in the statistical fitting procedure. These data and their experimental uncertainties are shown in Fig. 123 together with the Padé fit ( $\left.L=9, N=57, \chi^{2}=1.21\right)$ and estimated uncertainty in percentages, including $4 \%$ systematic uncertainty (righthand scale).
Fig. 122 Seven experimental datasets for the ${ }^{94} \mathrm{Mo}(p, n)^{94 \mathrm{~m}} \mathrm{Tc}$ reaction available in the literature [36, 142, 271-275], and TENDL calculations

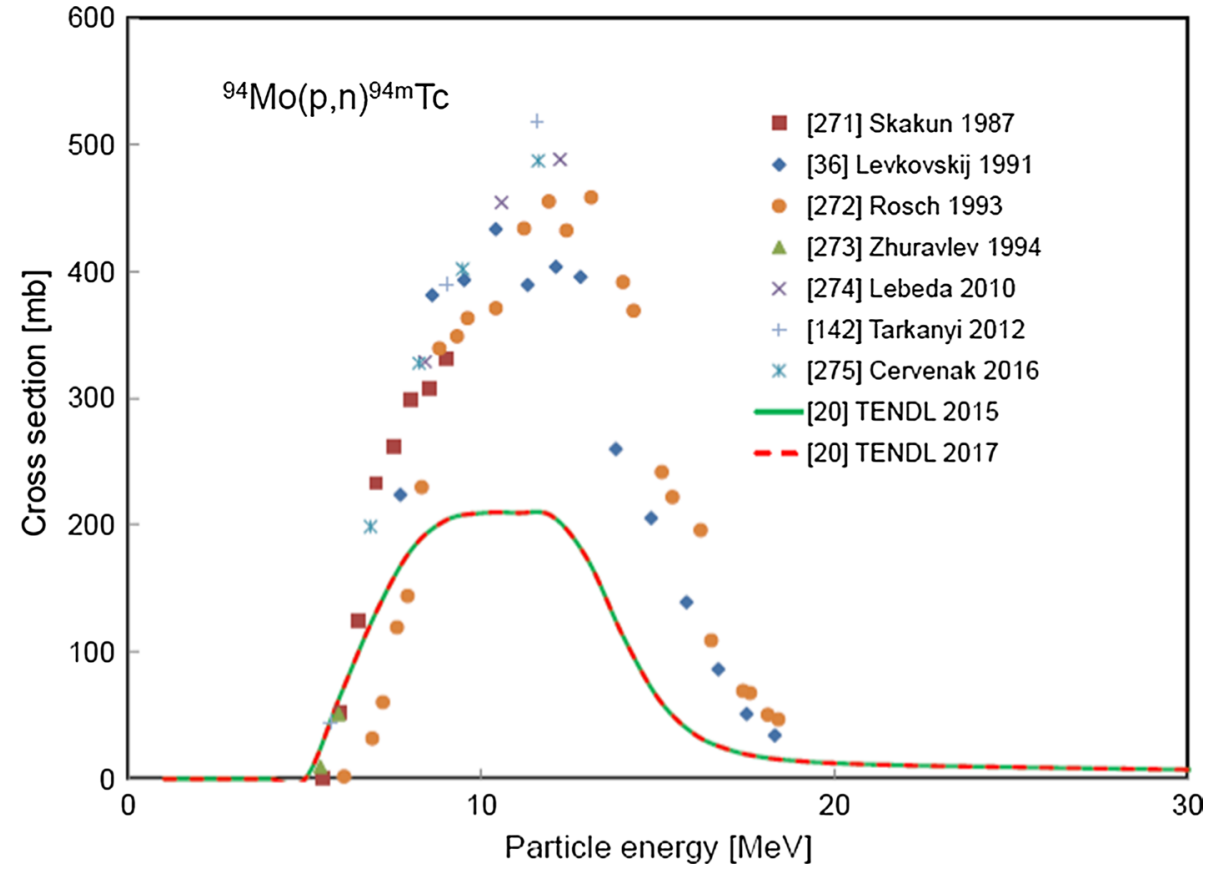

Fig. 123 Seven experimental datasets for the ${ }^{94} \mathrm{Mo}(\mathrm{p}, \mathrm{n})^{94 \mathrm{~m}} \mathrm{Tc}$ reaction [36, 142, 271-275] with the Padé fit $(L=9, N=57$, $\chi^{2}=1.21$, solid line) and estimated total uncertainties in percentages, including $4 \%$ systematic uncertainty (dashed line, right-hand scale)

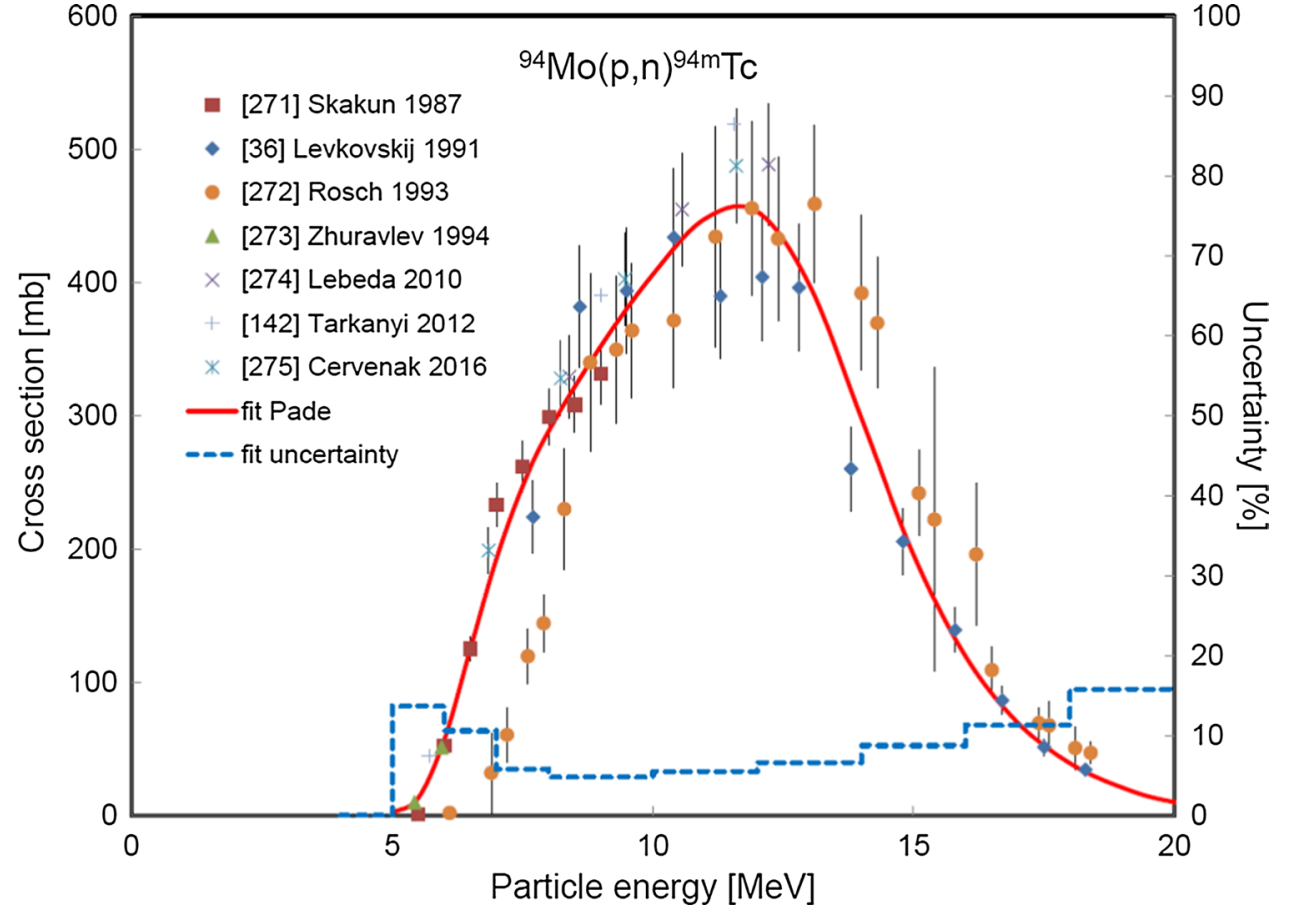


Thick target yields for production of ${ }^{94 \mathrm{~m}} \mathrm{Tc}$

See Fig. 124.

Fig. 124 Thick target yields calculated from the recommended cross sections for the ${ }^{92} \mathrm{Mo}(\alpha, \mathrm{x})^{94 \mathrm{~m}} \mathrm{Tc}$ and

${ }^{94} \mathrm{Mo}(\mathrm{p}, \mathrm{n}){ }^{94 \mathrm{~m}} \mathrm{Tc}$ reactions

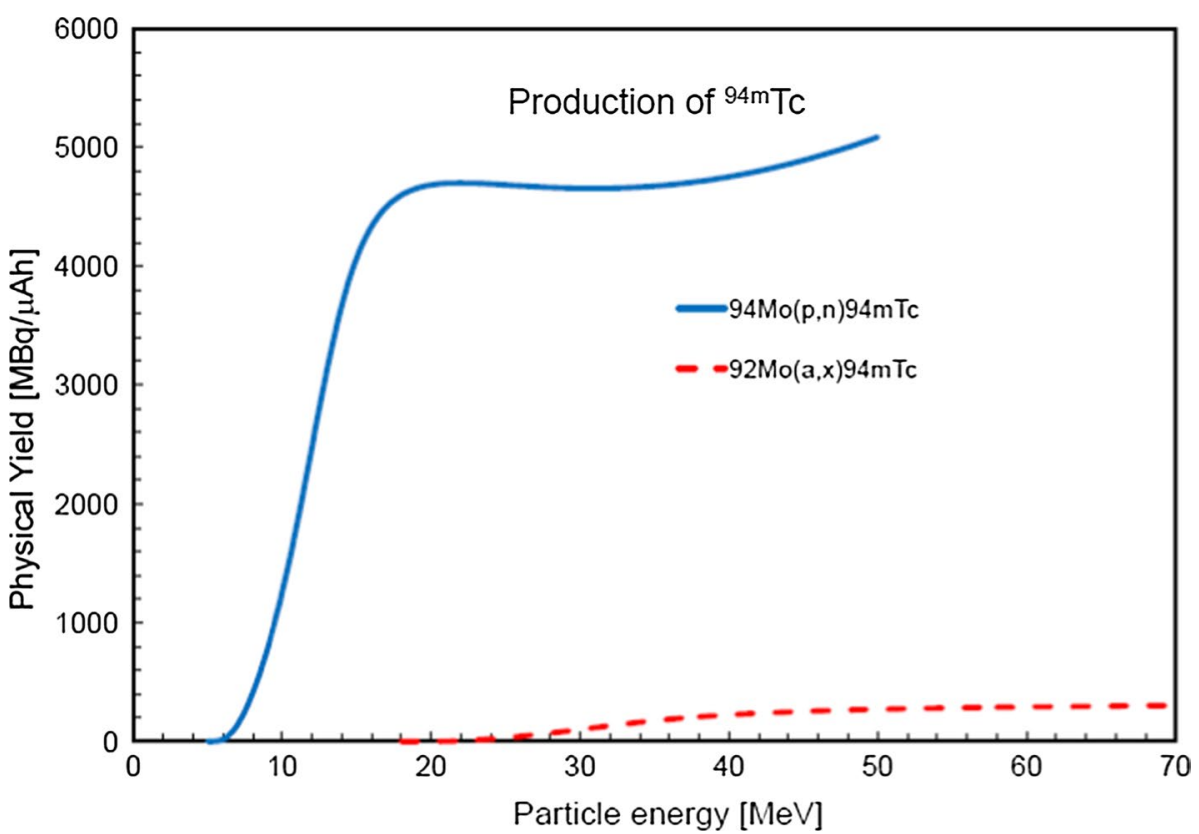


Production of ${ }^{110 \mathrm{~m}} \ln \left(T_{1 / 2}=69.1 \mathrm{~min}\right)$

and longer-lived ${ }^{110} \mathrm{Sn}$ parent $\left(T_{1 / 2}=4.154 \mathrm{~h}\right)$

Applications: ${ }^{110 \mathrm{~m}} \mathrm{In}$ is a positron-emitting analogue for established SPECT ${ }^{111}$ In. Potential to provide more quantitative diagnostic information as well as in vivo quantification of the uptake kinetics of radiopharmaceuticals (e.g., applied along with ${ }^{111}$ In-labelled DTPA-D-Phe1-octeotride for neuroendocrine tumours).

${ }^{110 \mathrm{~m}}$ In can be produced directly and via parent ${ }^{110} \mathrm{Sn}$.

${ }^{110 m} \operatorname{In}(69.1 \mathrm{~min}): \beta^{+}(61.3 \%)$, and $E_{\gamma}(\mathrm{keV})\left(P_{\gamma}(\%)\right): 2129.40$ (2.15), 2211.33 (1.74), 2317.41 (1.285).

${ }^{110} \mathrm{Sn}(4.154 \mathrm{~h}): E_{\gamma}(\mathrm{keV})\left(P_{\gamma}(\%)\right): 280.459$ (97.06).
Evaluations have been made of the ${ }^{\text {nat }} \operatorname{In}(\mathrm{p}, \mathrm{xn}){ }^{110} \mathrm{Sn}$, ${ }^{108} \mathrm{Cd}(\alpha, 2 \mathrm{n}){ }^{110} \mathrm{Sn},{ }^{110} \mathrm{Cd}(\mathrm{p}, \mathrm{n}){ }^{110 \mathrm{~m}} \mathrm{In},{ }^{110} \mathrm{Cd}(\mathrm{d}, 2 \mathrm{n}){ }^{110 \mathrm{~m}} \mathrm{In}$ and ${ }^{107} \mathrm{Ag}(\alpha, \mathrm{n}){ }^{110 \mathrm{~m}}$ In production routes.

${ }^{n a t} \ln (p, x n){ }^{110} S n$

The four experimental datasets available in the literature are shown in Fig. 125 [276-279] together with the TENDL calculations. All datasets were used in the statistical fitting procedure. The data and their experimental uncertainties are shown in Fig. 126 together with the Padé fit $(L=17, N=112$, $\left.\chi^{2}=1.50\right)$ and estimated uncertainty in percentages, including $4 \%$ systematic uncertainty (right-hand scale).
Fig. 125 Four experimental datasets for the ${ }^{\text {nat }} \operatorname{In}(\mathrm{p}, \mathrm{xn}){ }^{110} \mathrm{Sn}$ reaction available in the literature [276-279], and TENDL calculations

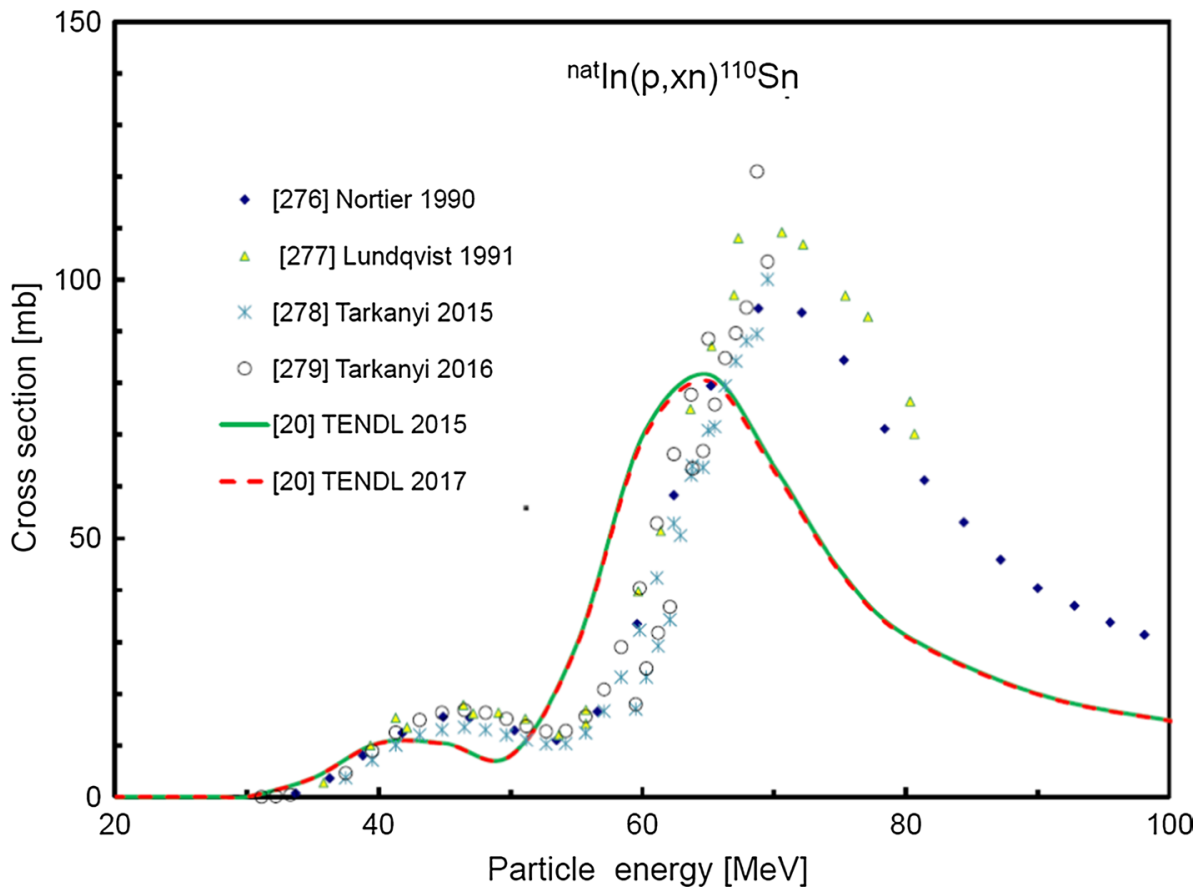


Fig. 126 Four experimental datasets for the ${ }^{\text {nat }} \operatorname{In}(\mathrm{p}, \mathrm{xn}){ }^{110} \mathrm{Sn}$ reaction [276-279] with the Padé fit $(L=17, N=112$, $\chi^{2}=1.50$, solid line) and estimated total uncertainties in percentages, including $4 \%$ systematic uncertainty (dashed line, right-hand scale)

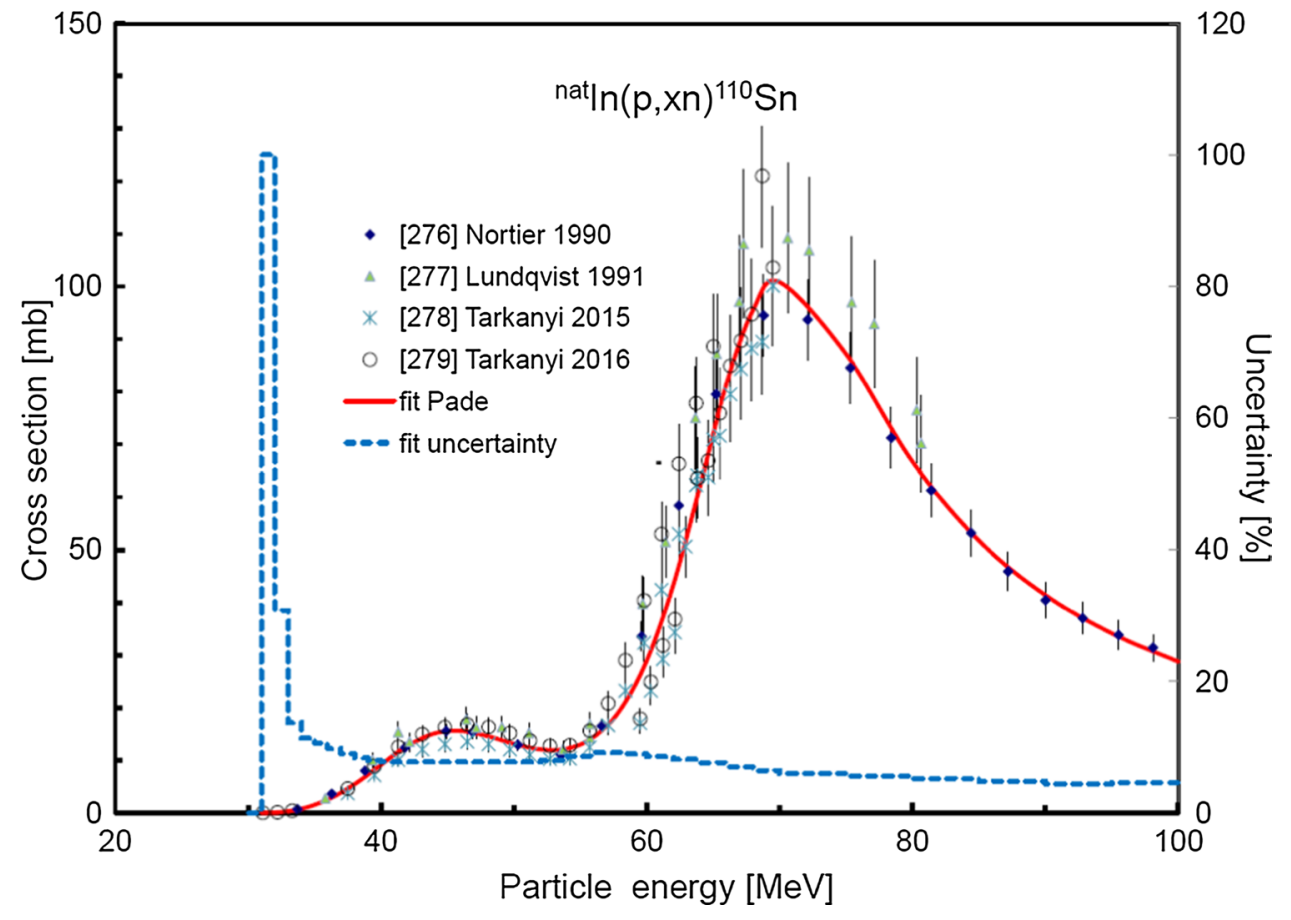


${ }^{108} \mathrm{Cd}(a, 2 n){ }^{110} \mathrm{Sn}$

The four experimental datasets available in the literature are shown in Fig. 127 [280-283] together with the TENDL calculations. One dataset was rejected (Duchemin et al. [282], values too low), while another became available after the evaluation cut-off date and therefore was not included (Ditrói et al. [283]). The two remaining sets were used in the statistical fitting procedure, and these selected data and their experimental uncertainties are shown in Fig. 128 together with the Padé fit $\left(L=10, N=24, \chi^{2}=1.99\right)$ and estimated uncertainty in percentages, including $4 \%$ systematic uncertainty (right-hand scale).
Fig. 127 Four experimental datasets for the ${ }^{108} \mathrm{Cd}(\alpha, 2 \mathrm{n}){ }^{110} \mathrm{Sn}$ reaction available in the literature [280-283], and TENDL calculations
Fig. 128 Two selected experimental datasets for the ${ }^{108} \mathrm{Cd}(\alpha, 2 \mathrm{n}){ }^{110} \mathrm{Sn}$ reaction $[280$, $281]$ with the Padé fit $(L=10$, $N=24, \chi^{2}=1.99$, solid line) and estimated total uncertainties in percentages, including $4 \%$ systematic uncertainty (dashed line, right-hand scale)
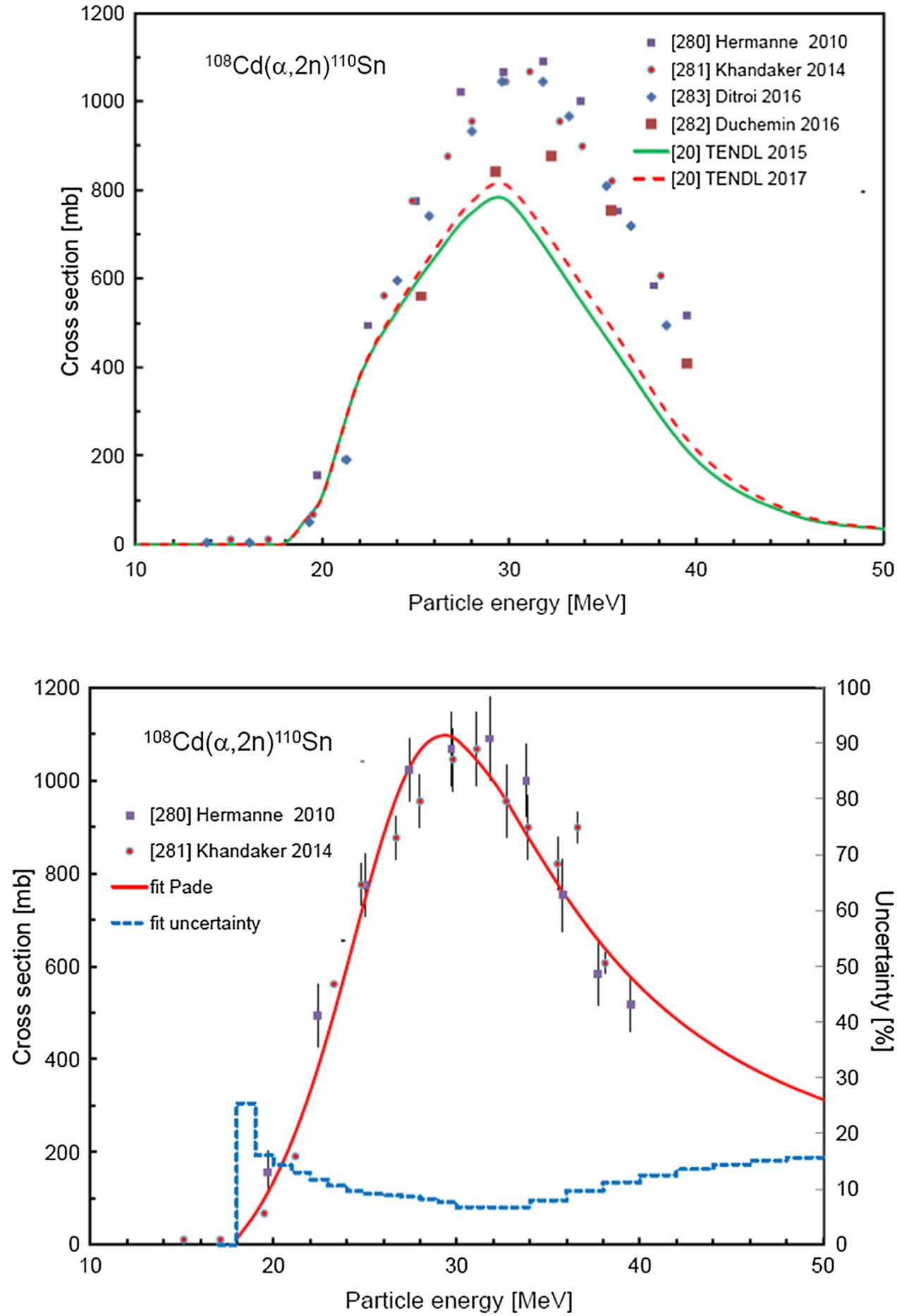


\section{${ }^{110} \mathrm{Cd}(p, n){ }^{110 \mathrm{~m}} \mathrm{In}$}

The three experimental datasets available in the literature for production of the metastable state $[164,284,285]$ and the six datasets for simultaneously produced impurity ground state [276, 285-289] are shown in Fig. 129 together with the TENDL calculations for the separate metastable and ground states. After full assessment, the three datasets were used in the statistical fitting procedure for the metastable state [164, 284, 285]. These data and their experimental uncertainties are shown in Fig. 130 together with the Padé fit $(L=11$, $\left.N=29, \chi^{2}=0.57\right)$ and estimated uncertainty in percentages, including $4 \%$ systematic uncertainty (right-hand scale).
Fig. 129 Three experimental datasets for the ${ }^{110} \mathrm{Cd}(\mathrm{p}, \mathrm{n})^{110 \mathrm{~m}} \mathrm{In}$ reaction available in the literature [164, 284, 285], along with six experimental datasets for the ${ }^{110} \mathrm{Cd}(\mathrm{p}, \mathrm{n})^{110 \mathrm{~g}}$ In reaction [276, 285-289], and TENDL calculations

Fig. 130 Three experimental datasets for the ${ }^{110} \mathrm{Cd}(\mathrm{p}, \mathrm{n}){ }^{110 \mathrm{~m}} \mathrm{In}$ reaction [164, 284, 285] with the Padé fit $(L=11, N=29$, $\chi^{2}=0.57$, solid line) and estimated total uncertainties in percentages, including $4 \%$ systematic uncertainty (dashed line, right-hand scale)
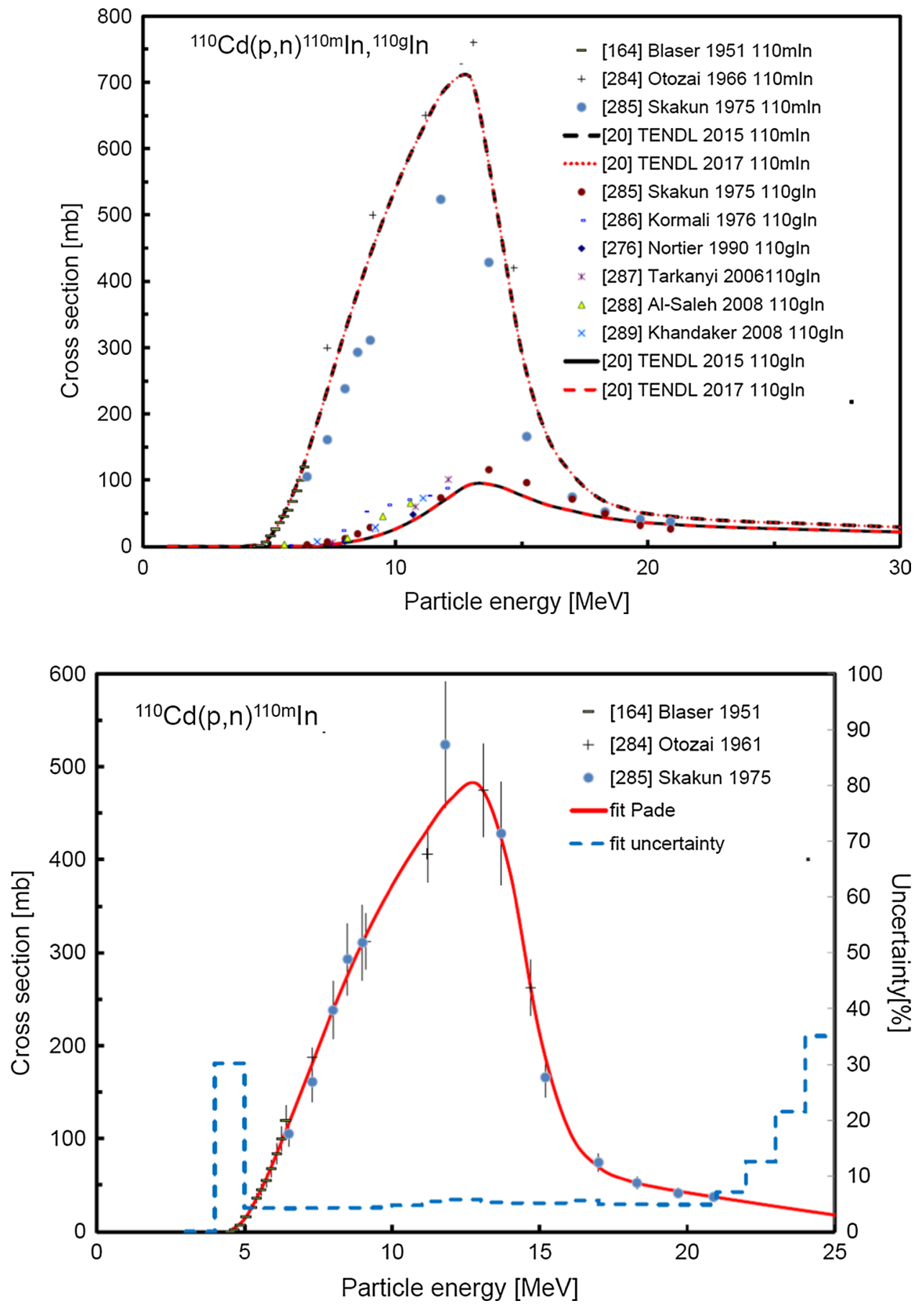


\section{${ }^{110} \mathrm{Cd}(\mathrm{d}, 2 \mathrm{n}){ }^{110 \mathrm{~m}} \mathrm{In}$}

The two experimental datasets available in the literature for production of the metastable state are shown in Fig. 131 [290, 291] together with two datasets for simultaneous production of the contaminating ground state [291, 292] and the TENDL calculations. Cross sections determined by Usher et al. [290] were normalised to the data of Tárkányi et al. [291], and both datasets were used in the statistical fitting procedure for ${ }^{110 \mathrm{~m}}$ In production. These data and their experimental uncertainties are shown in Fig. 132 together with the Padé fit $\left(L=5, N=18, \chi^{2}=0.74\right)$ and estimated uncertainty in percentages, including $4 \%$ systematic uncertainty (righthand scale).
Fig. 131 Two experimental datasets for the ${ }^{110} \mathrm{Cd}(\mathrm{d}, 2 \mathrm{n})^{110 \mathrm{~m}}$ In reaction available in the literature [290, 291] and two experimental datasets for the contaminating ${ }^{110} \mathrm{Cd}(\mathrm{d}, 2 \mathrm{n}){ }^{110 \mathrm{~g}}$ In reaction [291, 292], and their TENDL calculations. All data from Ref. [290] have been normalised with respect to the data of Ref. [291]

Fig. 132 Two experimental datasets for the

${ }^{110} \mathrm{Cd}(\mathrm{d}, 2 \mathrm{n}){ }^{110 \mathrm{~m}} \mathrm{In}$ reaction $[290$, 291] with the Padé fit ( $L=5$, $N=18, \chi^{2}=0.74$, solid line) and estimated total uncertainties in percentages, including $4 \%$ systematic uncertainty (dashed line, right-hand scale)
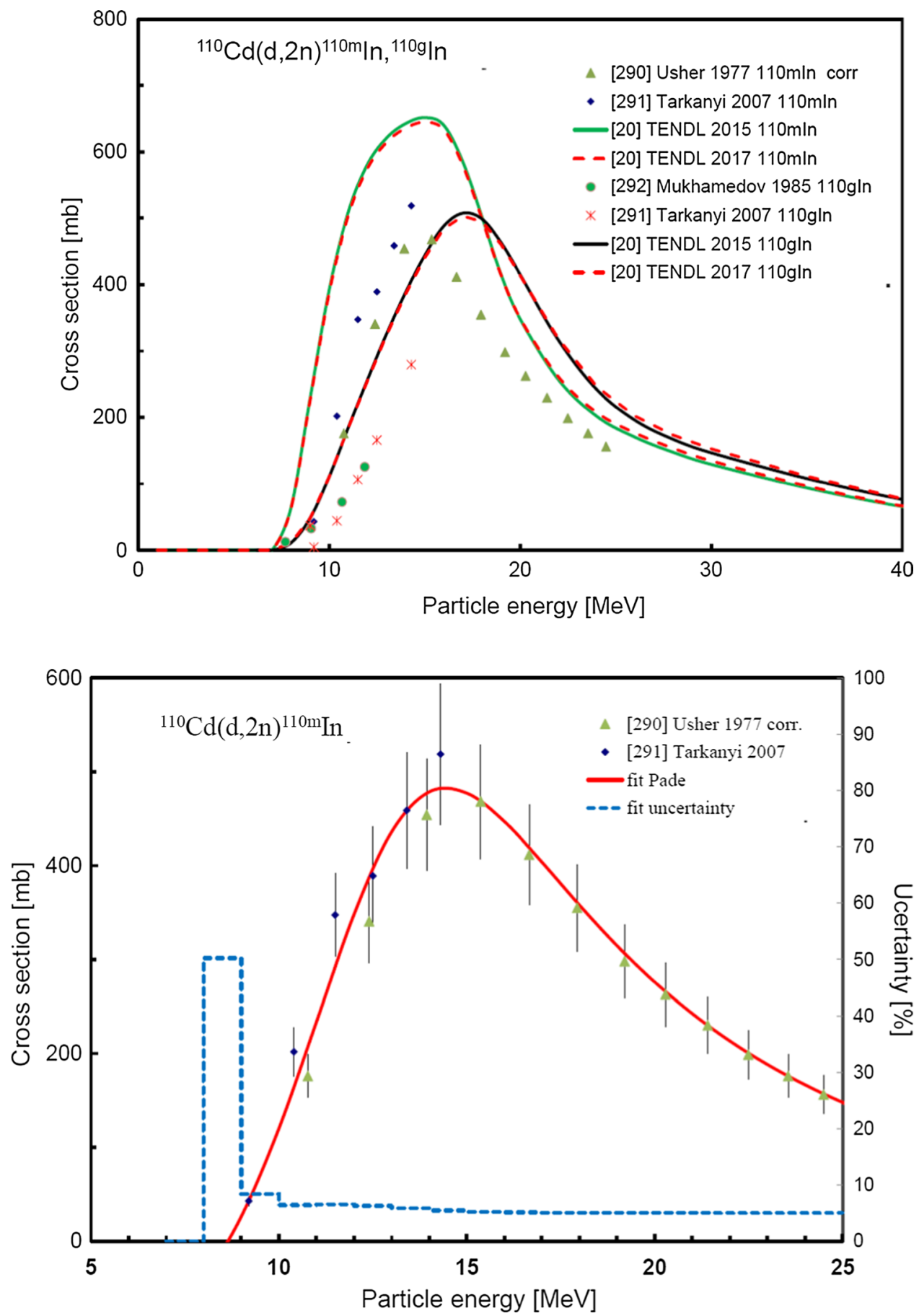


\section{${ }^{107} \mathrm{Ag}(\mathbf{a}, \mathrm{n}){ }^{110 \mathrm{~m}} \mathrm{In}$}

The nine experimental datasets available in the literature are shown in Fig. 133 [178, 278, 293-299] together with the TENDL calculations. Six datasets were rejected (Wasilevsky et al. [295] (discrepant values), Misaelides and Münzel [294] (energy steps too large, can not be controlled), Chaubey et al. [296] (values too high), Fukushima et al. [293] (values too low at higher energy), Patel et al. [297] (values too low), Takács et al. [298] (values too low)), and the remaining three sets were used in the statistical fitting procedure. The selected data and their experimental uncertainties are shown in Fig. 134 together with the Padé fit $L=9, N=32$, $\left.\chi^{2}=1.94\right)$ and estimated uncertainty in percentages, including $4 \%$ systematic uncertainty (right-hand scale).
Fig. 133 Nine experimental datasets for the ${ }^{107} \mathrm{Ag}(\alpha, n)^{110 \mathrm{~m}} \mathrm{In}$ reaction available in the literature [178, 278, 293-299], and TENDL calculations
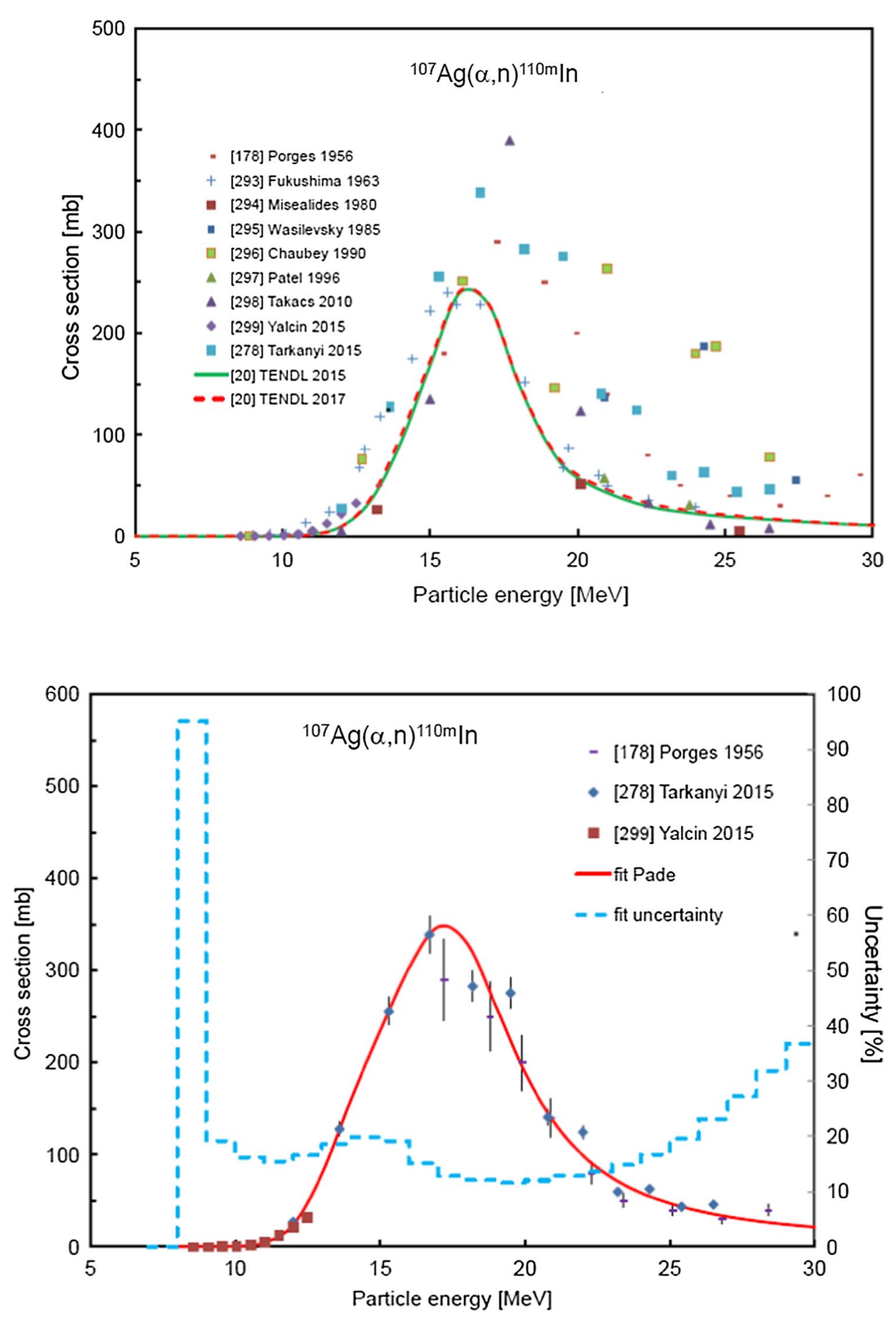

Fig. 134 Three selected experimental datasets for the ${ }^{107} \mathrm{Ag}(\alpha, \mathrm{n}){ }^{110 \mathrm{~m}}$ In reaction [178, 278, 299] with the Padé fit $\left(L=9, N=32, \chi^{2}=1.94\right.$, solid line) and estimated total uncertainties in percentages, including $4 \%$ systematic uncertainty (dashed line, right-hand scale) 


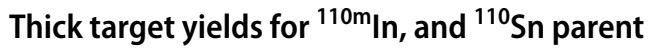

See Figs. 135 and 136.

Fig. 135 Thick target yields calculated from the recommended cross sections for ${ }^{110} \mathrm{Cd}(\mathrm{p}, \mathrm{n}){ }^{110 \mathrm{~m}} \mathrm{In}$, ${ }^{110} \mathrm{Cd}(\mathrm{d}, 2 \mathrm{n}){ }^{110 \mathrm{~m}}$ In and ${ }^{107} \mathrm{Ag}(\alpha, \mathrm{n}){ }^{110 \mathrm{~m}}$ In reactions
Fig. 136 Thick target yields calculated from the recommended cross sections for the ${ }^{\text {nat }} \operatorname{In}(\mathrm{p}, \mathrm{xn}){ }^{110} \mathrm{Sn}$ and ${ }^{108} \mathrm{Cd}(\alpha, 2 \mathrm{n}){ }^{110} \mathrm{Sn}$ reactions
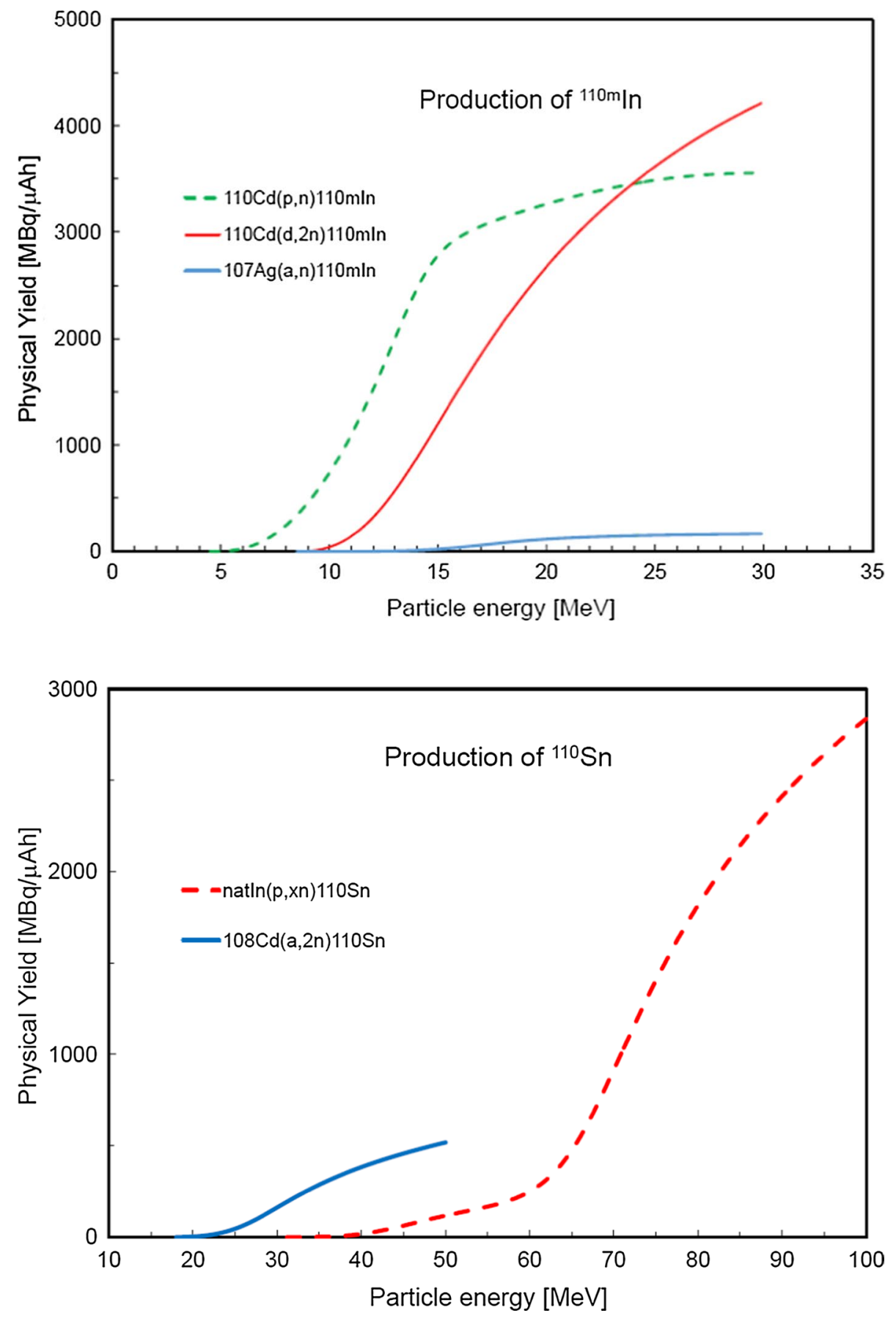


\section{Production of ${ }^{118} \mathrm{Te}$ parent $\left(T_{1 / 2}=6.00 \mathrm{~d}\right)$ of short-lived ${ }^{118} \mathrm{Sb}\left(T_{1 / 2}=3.6 \mathrm{~min}\right)$}

Applications: EC decay of ${ }^{118} \mathrm{Te}$ produces 3.6 min half-life ${ }^{118} \mathrm{Sb}$ daughter, which decays primarily by positron emission and can be used as a flow tracer.

${ }^{118} \mathrm{Sb}(3.6 \mathrm{~min}): \beta^{+}(73.5 \%)$, and $E_{\gamma}(\mathrm{keV})\left(P_{\gamma}(\%)\right): 1229.33$ (2.5).

${ }^{118} \mathrm{Te}(6.00 \mathrm{~d})$ : detected by means of radiation emitted by daughter ${ }^{118} \mathrm{Sb}$.

Evaluations have been undertaken for the ${ }^{115} \mathrm{Sn}(\alpha, \mathrm{n}){ }^{118} \mathrm{Te}$, ${ }^{116} \mathrm{Sn}(\alpha, 2 \mathrm{n}){ }^{118} \mathrm{Te},{ }^{\text {nat }} \mathrm{Sb}(\mathrm{p}, \mathrm{xn}){ }^{118} \mathrm{Te}$ and ${ }^{\mathrm{nat}} \mathrm{Sb}(\mathrm{d}, \mathrm{xn}){ }^{118} \mathrm{Te}$ production routes.
${ }^{115} \mathrm{Sn}(a, n){ }^{118} \mathrm{Te}$

The two experimental datasets available in the literature are shown in Fig. 137 [300, 301] together with the TENDL calculations. These two datasets were used as the basis of the fitting procedure. However, to obtain reasonable crosssection behaviour above $\sim 18 \mathrm{MeV}$, three artificial points were added in accord with the TENDL-2017 calculations for beam energies of 20,25 and $30 \mathrm{MeV}$ given assigned uncertainties of $25 \%$. The data and their experimental uncertainties are shown in Fig. 138 together with the Padé fit $(L=7$, $\left.N=8, \chi^{2}=1.07\right)$ and estimated uncertainty in percentages, including $4 \%$ systematic uncertainty (right-hand scale).
Fig. 137 Two experimental datasets for the ${ }^{115} \mathrm{Sn}(\alpha, \mathrm{n})^{118} \mathrm{Te}$ reaction available in the literature [300, 301], and TENDL calculations

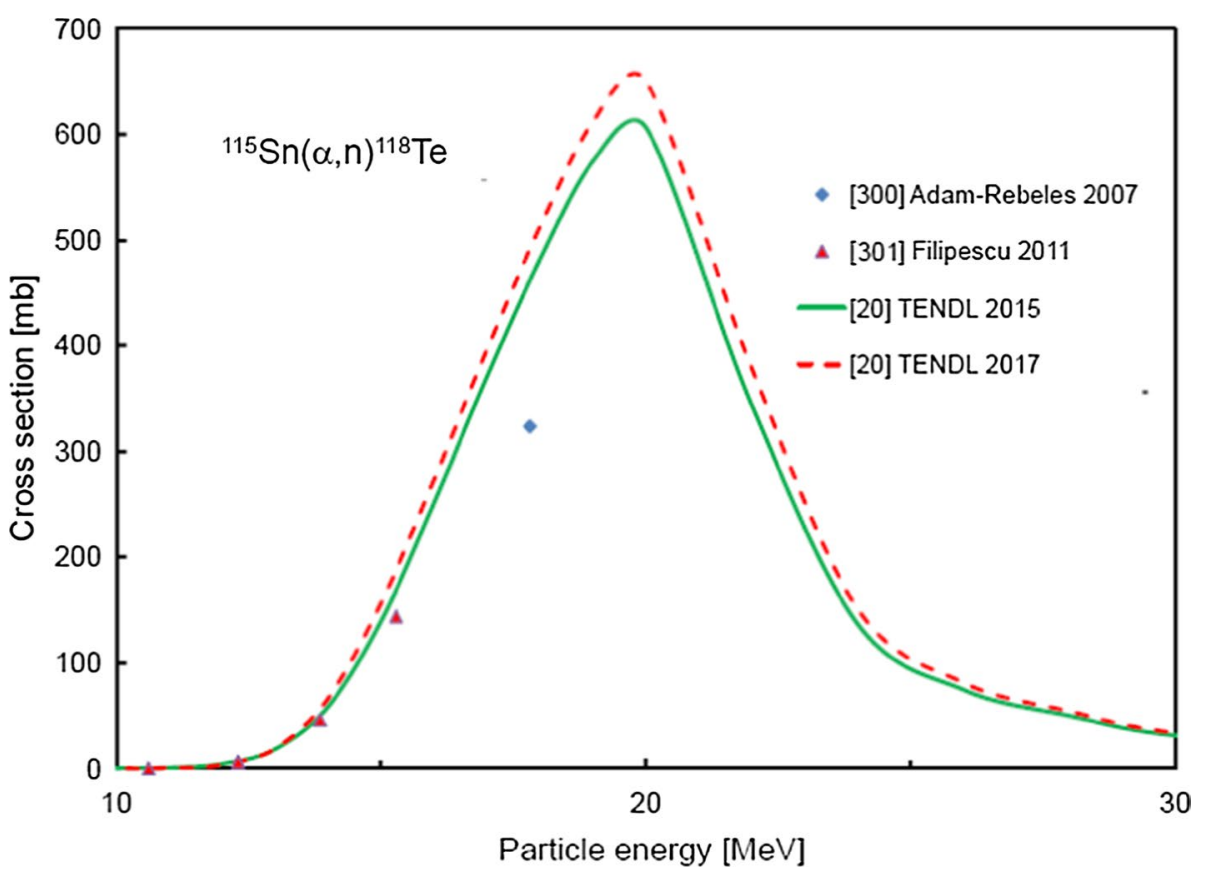


Fig. 138 Two experimental datasets for the ${ }^{115} \mathrm{Sn}(\alpha, \mathrm{n})^{118} \mathrm{Te}$ reaction $[300,301]$ with the

Padé fit $\left(L=7, N=8, \chi^{2}=1.07\right.$, solid line) and estimated total uncertainties in percentages, including 4\% systematic uncertainty (dashed line, right-hand scale)

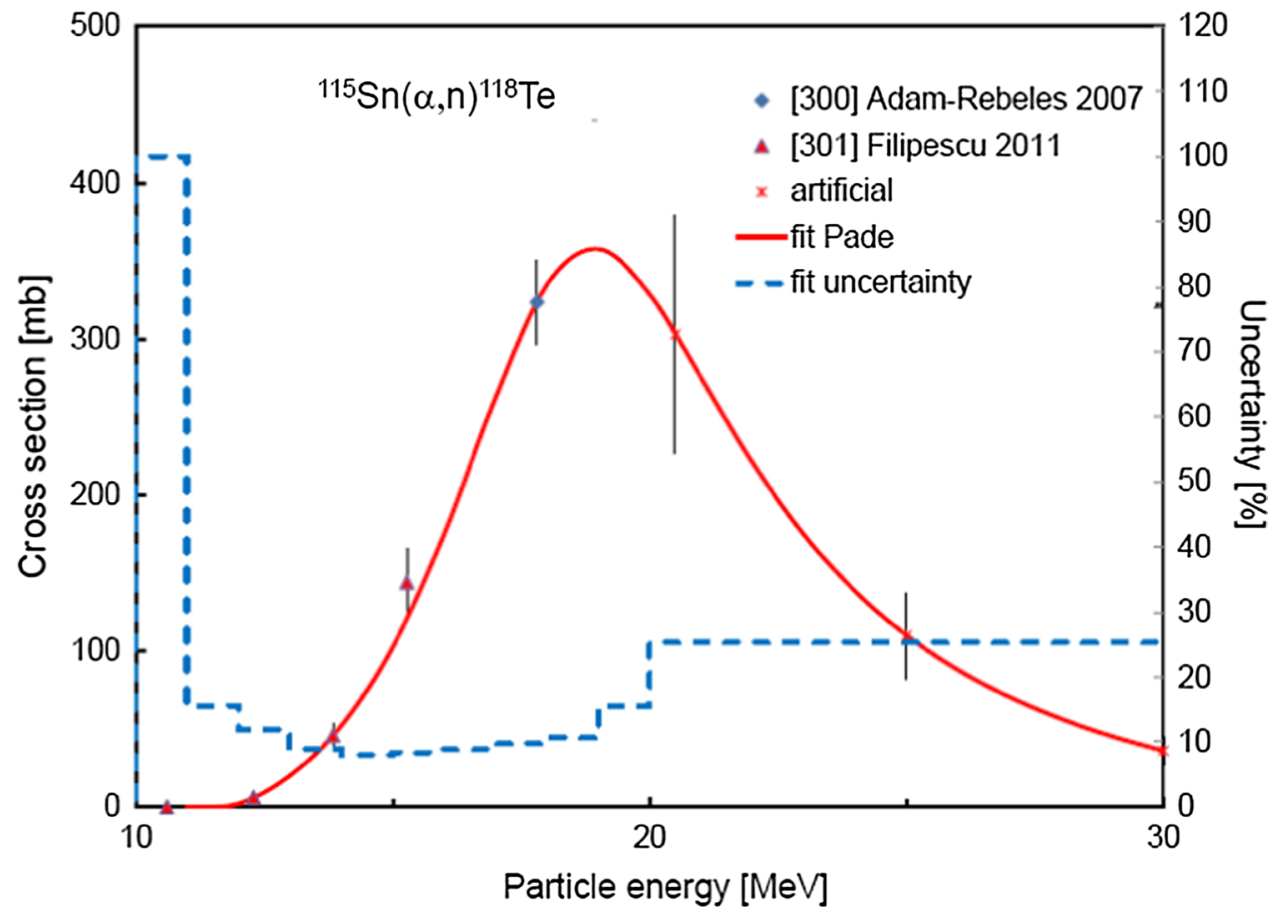




\section{${ }^{116} \mathrm{Sn}(a, 2 \mathrm{n}){ }^{118} \mathrm{Te}$}

The two experimental datasets available in the literature are shown in Fig. 139 [300, 302] together with the TENDL calculations. Both datasets were used in the statistical fitting procedure. The data and their experimental uncertainties are shown in Fig. 140 together with the Padé fit $(L=6, N=13$, $\left.\chi^{2}=1.34\right)$ and estimated uncertainty in percentages, including $4 \%$ systematic uncertainty (right-hand scale).
Fig. 139 Two experimental datasets for the ${ }^{116} \mathrm{Sn}(\alpha, 2 \mathrm{n}){ }^{118} \mathrm{Te}$ reaction available in the literature [300, 302], and TENDL calculations
Fig. 140 Two experimental datasets for the ${ }^{116} \mathrm{Sn}(\alpha, 2 \mathrm{n})^{118} \mathrm{Te}$ reaction $[300,302]$ with the Padé fit $(L=6, N=13$, $\chi^{2}=1.34$, solid line) and estimated total uncertainties in percentages, including $4 \%$ systematic uncertainty (dashed line, right-hand scale)
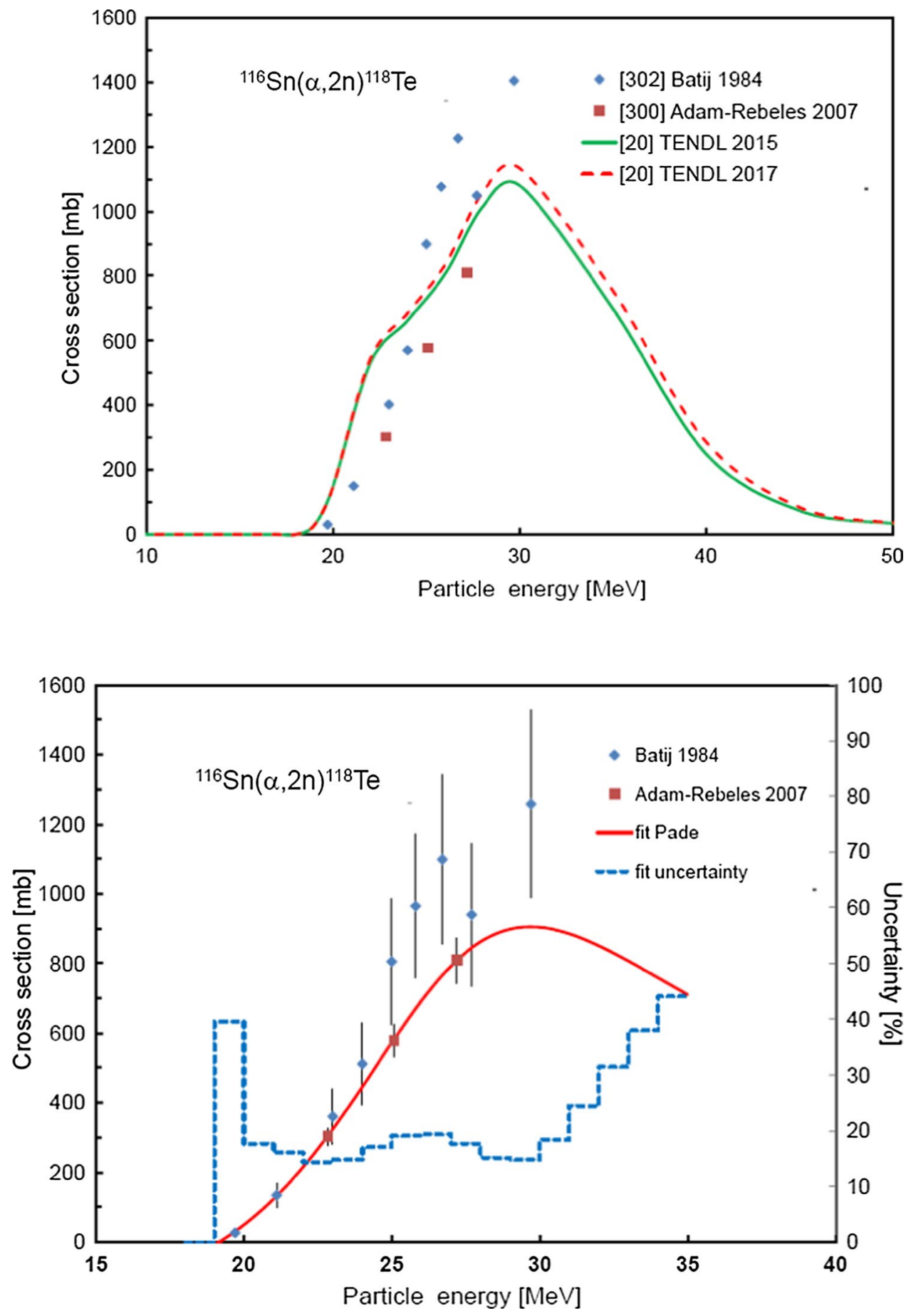


\section{${ }^{\text {nat }} \mathrm{Sb}(\mathrm{p}, \mathrm{xn}){ }^{118} \mathrm{Te}$}

The three experimental datasets available in the literature are shown in Fig. 141 [303-305] together with the TENDL calculations. One dataset was normalised and energy-shifted (Lagunas-Solar et al. [304]), and all three sets were used in the statistical fitting procedure. These data and their experimental uncertainties are shown in Fig. 142 together with the Padé fit $\left(L=12, N=43, \chi^{2}=0.53\right)$ and estimated uncertainty in percentages, including $4 \%$ systematic uncertainty (righthand scale). Contributions of the ${ }^{121} \mathrm{Sb}(\mathrm{p}, 4 \mathrm{n})$ and ${ }^{123} \mathrm{Sb}(\mathrm{p}, 6 \mathrm{n})$ reactions can clearly be distinguished.
Fig. 141 Three experimental datasets for the ${ }^{\text {nat }} \mathrm{Sb}(\mathrm{p}, \mathrm{xn}){ }^{118} \mathrm{Te}$ reaction available in the literature [303-305], and TENDL calculations
Fig. 142 Three experimental datasets for the ${ }^{\text {nat }} \mathrm{Sb}(\mathrm{p}, \mathrm{xn})^{118} \mathrm{Te}$ reaction [303-305] with the Padé fit $(L=12, N=43$, $\chi^{2}=0.53$, solid line) and estimated total uncertainties in percentages, including $4 \%$ systematic uncertainty (dashed line, right-hand scale) - dataset from Ref. [304] has been normalised and energy-shifted
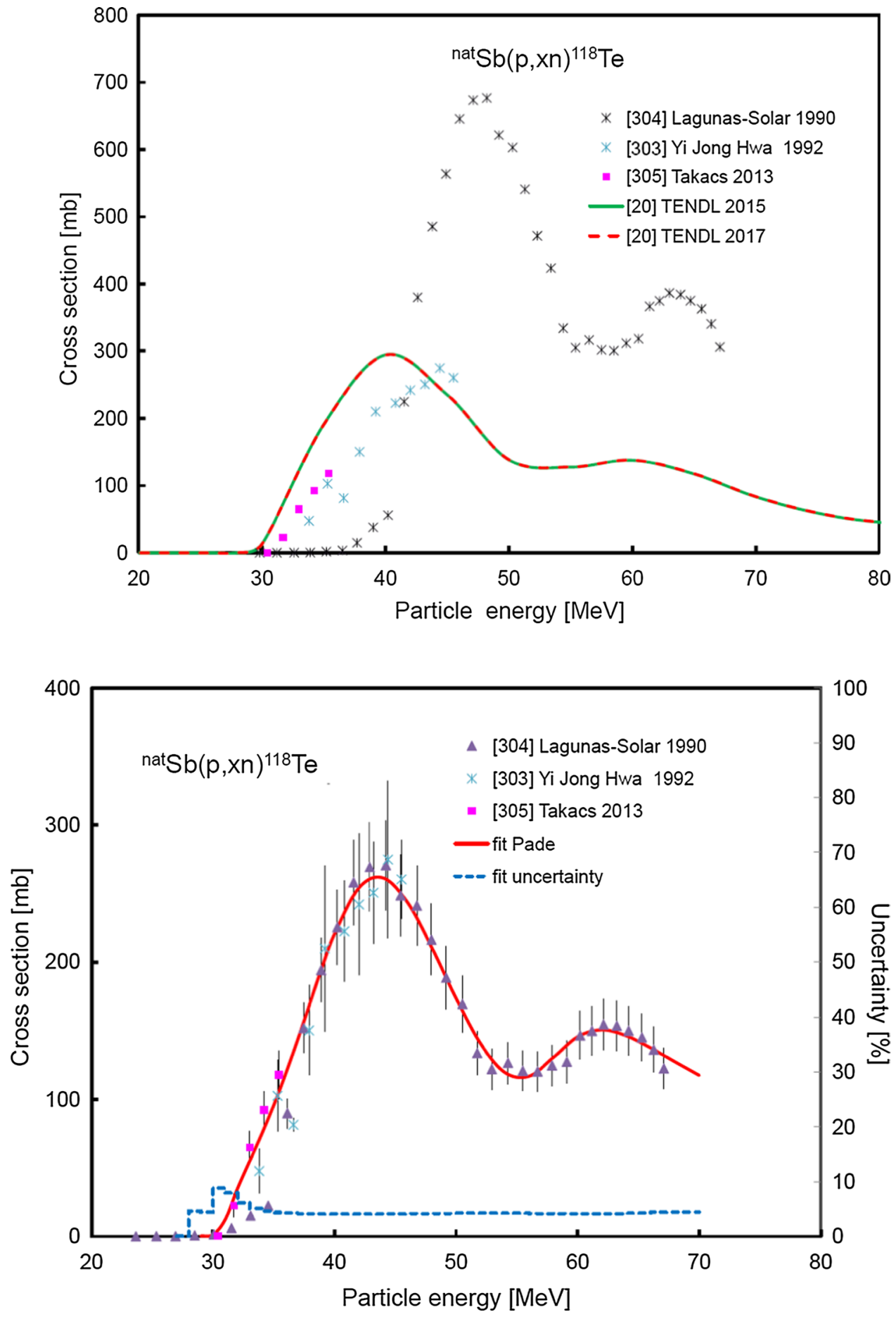


\section{${ }^{\text {nat }} \mathrm{Sb}(\mathrm{d}, \mathrm{xn})^{118} \mathrm{Te}$}

The single experimental dataset available in the literature is shown in Fig. 143 [306] together with the TENDL calculations. This one dataset was used in the statistical fitting procedure. The data and their experimental uncertainties are shown in Fig. 144 together with the Padé fit $(L=4, N=7$, $\left.\chi^{2}=0.52\right)$ and estimated uncertainty in percentages, including $4 \%$ systematic uncertainty (right-hand scale).
Fig. 143 One experimental dataset for the ${ }^{\text {nat }} \mathrm{Sb}(\mathrm{d}, \mathrm{xn}){ }^{118} \mathrm{Te}$ reaction available in the literature [306], and TENDL calculations
Fig. 144 One experimental dataset for the ${ }^{\text {nat }} \mathrm{Sb}(\mathrm{d}, \mathrm{xn})^{118} \mathrm{Te}$ reaction [306] with the Padé fit $\left(L=4, N=7, \chi^{2}=0.52\right.$, solid line) and estimated total uncertainties in percentages, including $4 \%$ systematic uncertainty (dashed line, right-hand scale)
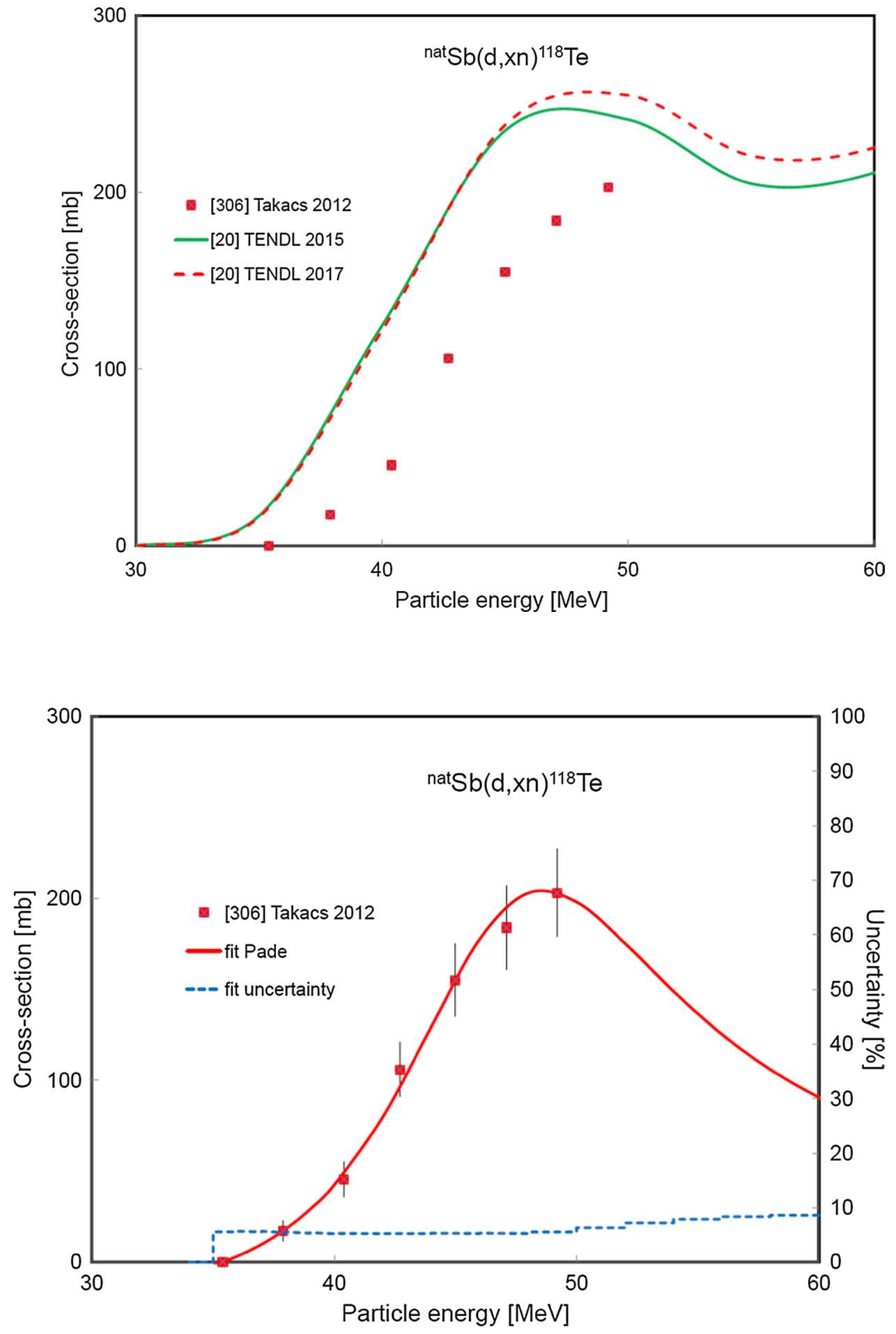
Thick target yields for production of ${ }^{118} \mathrm{Te}$ parent

of short-lived ${ }^{118} \mathrm{Sb}$

See Fig. 145.

Fig. 145 Thick target yields calculated from the rec-

ommended cross sections

for the ${ }^{115} \mathrm{Sn}(\alpha, \mathrm{n}){ }^{118} \mathrm{Te}$,

${ }^{116} \mathrm{Sn}(\alpha, 2 \mathrm{n}){ }^{118} \mathrm{Te}$,

${ }^{n a t} \mathrm{Sb}(\mathrm{p}, \mathrm{xn})^{118} \mathrm{Te}$ and

${ }^{\text {nat }} \mathrm{Sb}(\mathrm{d}, \mathrm{xn}){ }^{118} \mathrm{Te}$ reactions

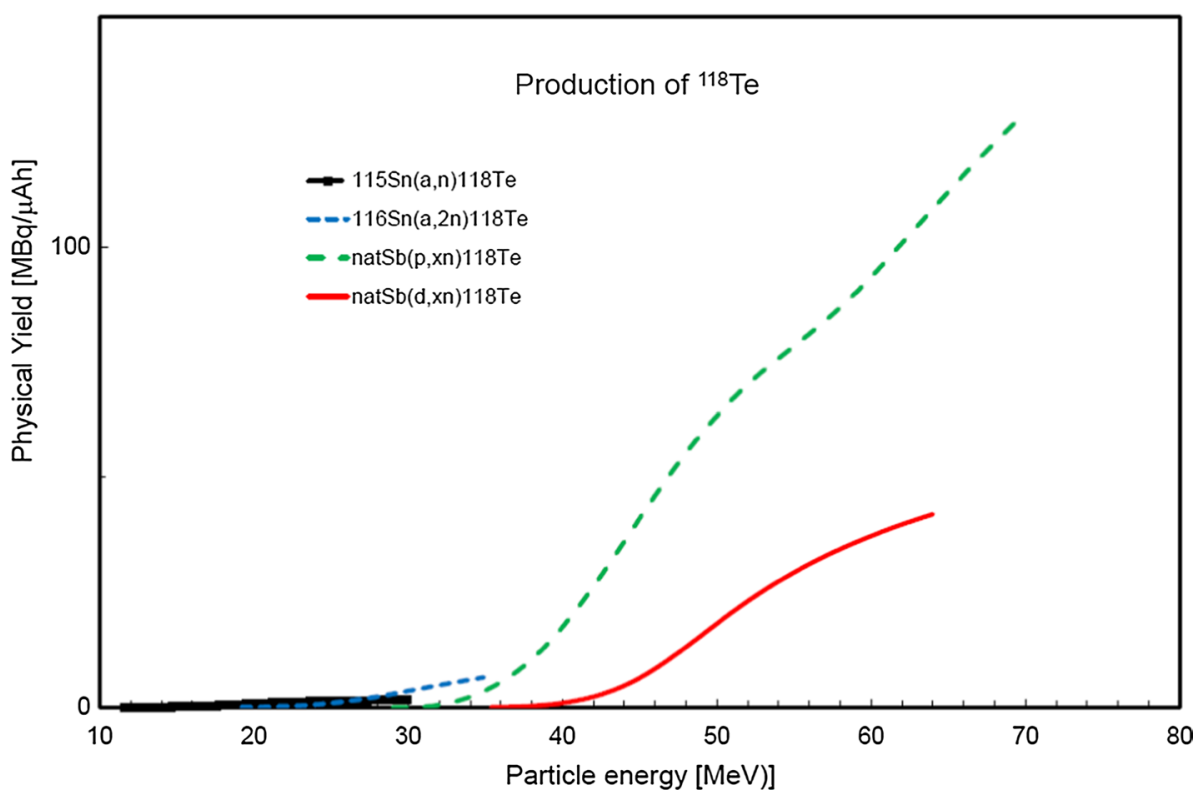




\section{Production of ${ }^{120} \mathrm{I}\left(T_{1 / 2}=81.6 \mathrm{~min}\right)$}

Applications: Positron-emitting ${ }^{120} \mathrm{I}\left(T_{1 / 2}=81.6 \mathrm{~min}\right)$ is a short-lived alternative to ${ }^{124} \mathrm{I}$ and ${ }^{123} \mathrm{I}$. This iodine radionuclide has a positron abundance more than twice that of ${ }^{124} \mathrm{I}$ and a maximum positron energy of $4.593 \mathrm{MeV}$. Can be used for radiohalogenation of molecules with rapid kinetics.

${ }^{120} I(81.6 \mathrm{~min}): \beta^{+}(68.2 \%)$, and $E_{\gamma}(\mathrm{keV})\left(P_{\gamma}(\%)\right): 601.1$ (5.51). 1523.0 (10.9).

Evaluations have been made of the ${ }^{120} \mathrm{Te}(\mathrm{p}, \mathrm{n}){ }^{120} \mathrm{I}$ and ${ }^{122} \mathrm{Te}(\mathrm{p}, 3 \mathrm{n}){ }^{120} \mathrm{I}$ reactions.
${ }^{120} \mathrm{Te}(\mathrm{p}, \mathrm{n}){ }^{120} \mathrm{I}$

The four experimental datasets available in the literature are shown in Fig. 146 [307-310] together with the TENDL calculations. Two datasets were rejected (El-Azony et al. [308] and Ahmed et al. [310], both exhibit energy shift around $10 \mathrm{MeV}$ ), and the remaining two datasets were used in the statistical fitting procedure. The selected data and their experimental uncertainties are shown in Fig. 147 together with the Padé fit ( $\left.L=18, N=38, \chi^{2}=0.806\right)$ and estimated uncertainty in percentages, including $4 \%$ systematic uncertainty (right-hand scale).
Fig. 146 Four experimental datasets for the ${ }^{120} \mathrm{Te}(\mathrm{p}, \mathrm{n}){ }^{120} \mathrm{I}$ reaction available in the literature [307-310], and TENDL calculations

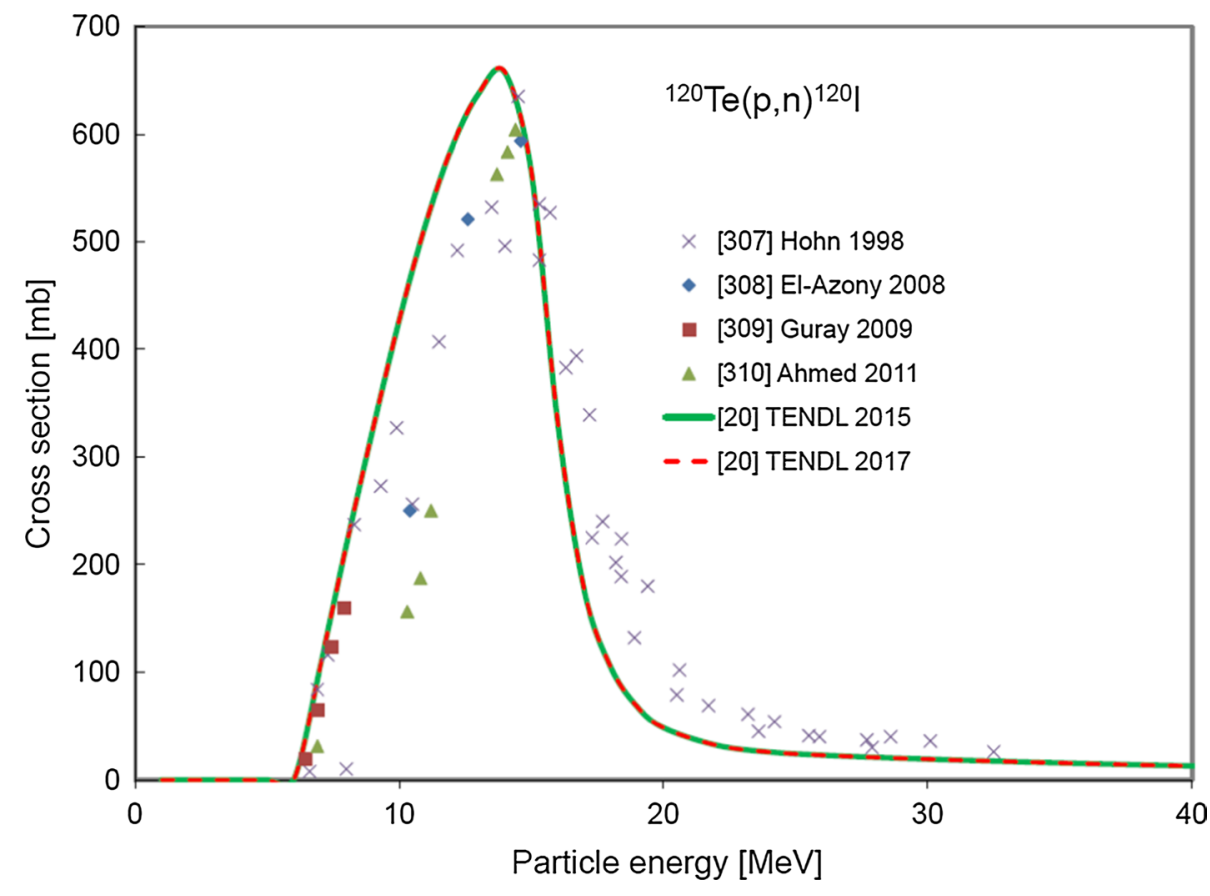


Fig. 147 Two selected experimental datasets for the ${ }^{120} \mathrm{Te}(\mathrm{p}, \mathrm{n}){ }^{120} \mathrm{I}$ reaction $[307$, 309] with the Padé fit $(L=18$, $N=38, \chi^{2}=0.806$, solid line) and estimated total uncertainties in percentages, including $4 \%$ systematic uncertainty (dashed line, right-hand scale)

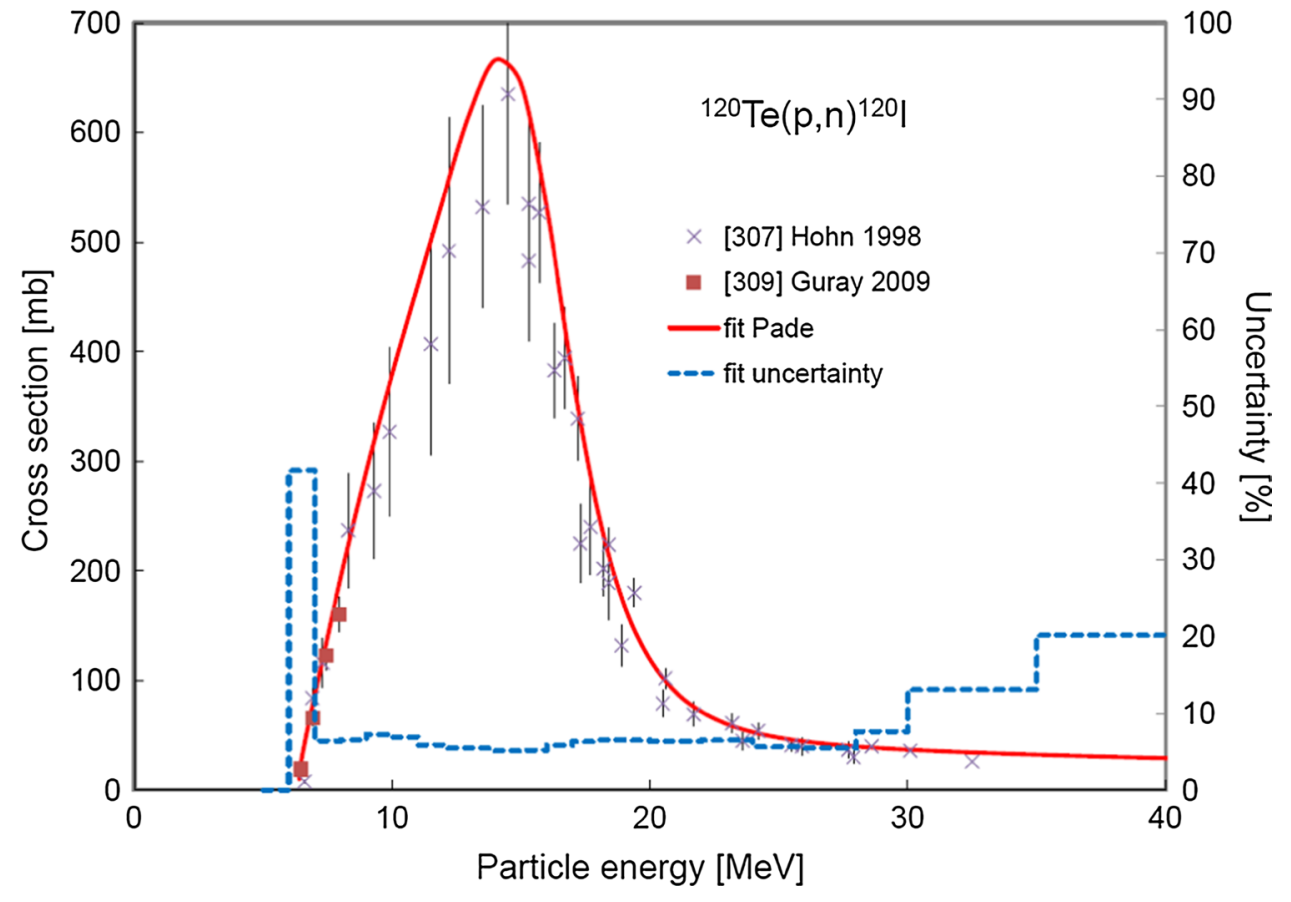




\section{${ }^{122} \mathrm{Te}(p, 3 n){ }^{120} \mathrm{I}$}

The two experimental datasets available in the literature are shown in Fig. 148 [307, 308] together with the TENDL calculations. These two datasets were used in the statistical fitting procedure. The data and their experimental uncertainties are shown in Fig. 149 together with the Padé fit $(L=7$, $\left.N=18, \chi^{2}=0.463\right)$ and estimated uncertainty in percentages, including $4 \%$ systematic uncertainty (right-hand scale).
Fig. 148 Two experimental datasets for the ${ }^{122} \mathrm{Te}(\mathrm{p}, 3 \mathrm{n}){ }^{120} \mathrm{I}$ reaction available in the literature [307, 308], and TENDL calculations
Fig. 149 Two experimental datasets for the ${ }^{122} \mathrm{Te}(\mathrm{p}, 3 \mathrm{n}){ }^{120} \mathrm{I}$ reaction $[307,308]$ with the Padé fit $(L=7, N=18$, $\chi^{2}=0.463$, solid line) and estimated total uncertainties in percentages, including $4 \%$ systematic uncertainty (dashed line, right-hand scale)
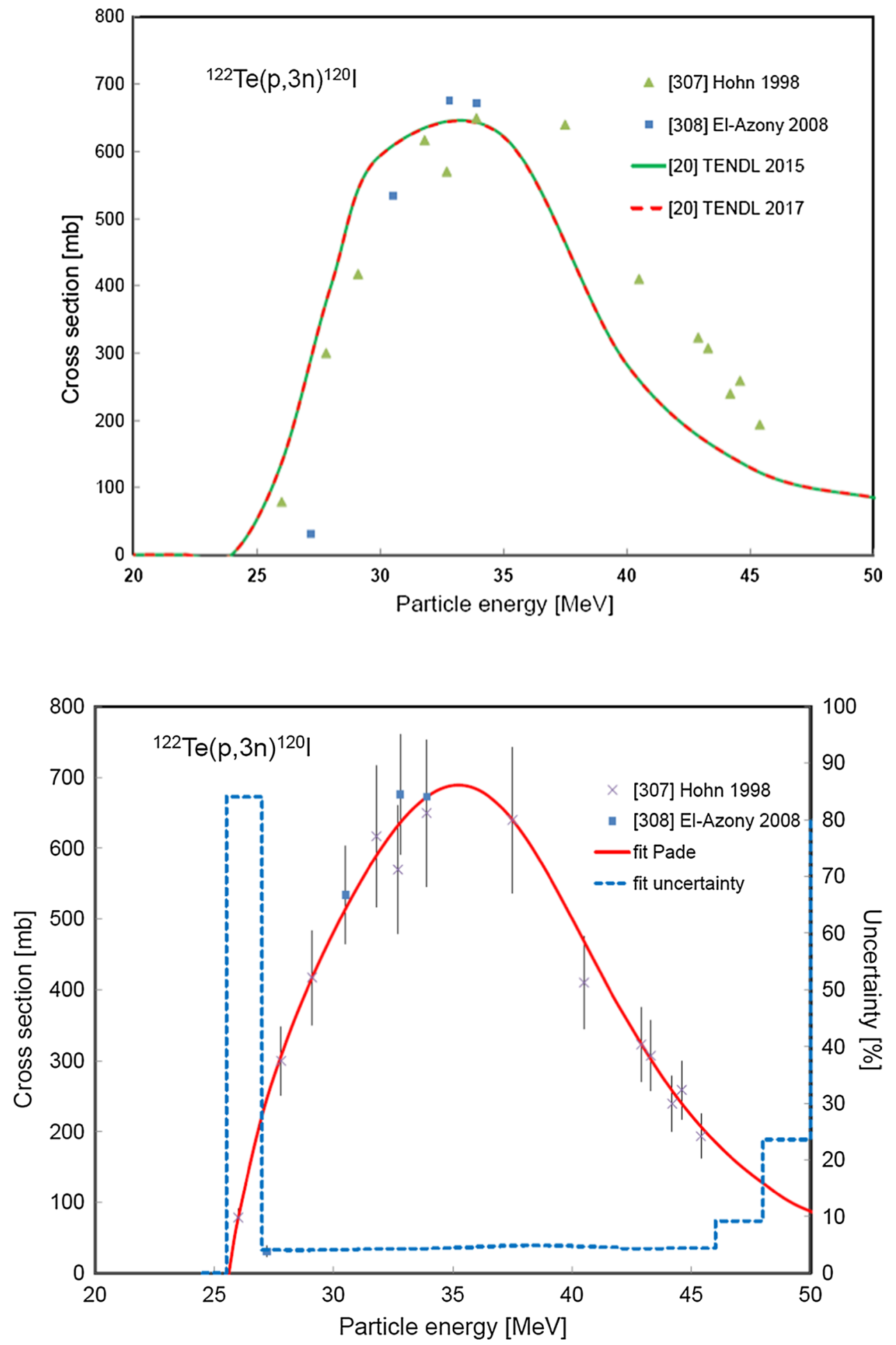


\section{Thick target yields for production of ${ }^{120}$ I}

See Fig. 150.

Fig. 150 Thick target yields calculated from the recommended cross sections for the ${ }^{120} \mathrm{Te}(\mathrm{p}, \mathrm{n})^{120} \mathrm{I}$ and ${ }^{122} \mathrm{Te}(\mathrm{p}, 3 \mathrm{n}){ }^{120} \mathrm{I}$ reactions

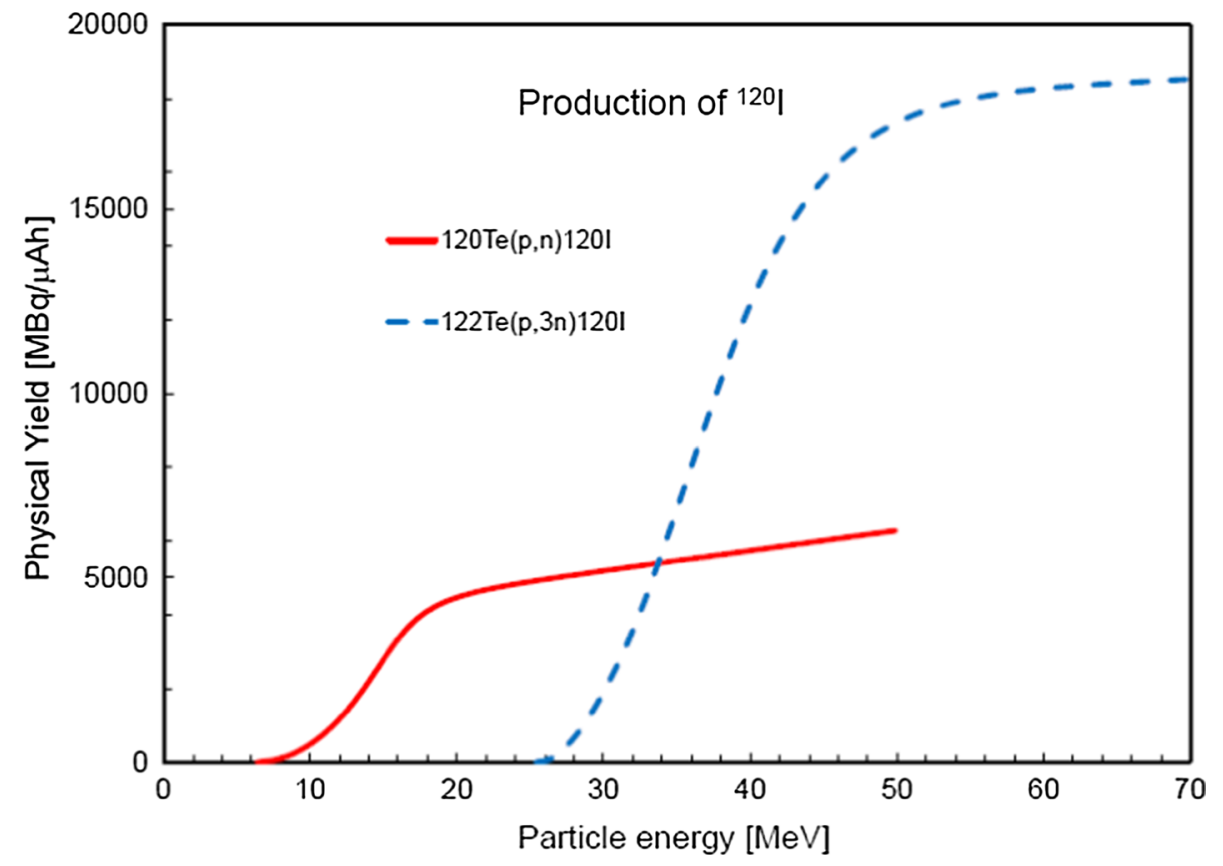


Production of ${ }^{122}$ Xe parent $\left(T_{1 / 2}=20.1 \mathrm{~h}\right)$ of short-lived $^{122}$ I $\left(T_{1 / 2}=3.63 \mathrm{~min}\right)$

Applications: ${ }^{122} \mathrm{I}$ with a half-life of $3.63 \mathrm{~min}$. has potential as a generator-produced positron emitter for various PET studies such as brain and heart perfusion.

${ }^{122} I(3.63 \mathrm{~min})$ : detected through $\beta^{+}(78 \%)$, and $E_{\gamma}(\mathrm{keV})$ $\left(P_{\gamma}(\%)\right): 564.119(18)$.

${ }^{122} \mathrm{Xe}(20.1 \mathrm{~h}): E_{\gamma}(\mathrm{keV})\left(P_{\gamma}(\%)\right): 350.065$ (7.80), and radiation emitted by daughter ${ }^{122} \mathrm{I}$.

Evaluations have been undertaken of the ${ }^{124} \mathrm{Xe}(\mathrm{p}, \mathrm{x})^{122} \mathrm{Xe}$, ${ }^{127} \mathrm{I}(\mathrm{p}, 6 \mathrm{n}){ }^{122} \mathrm{Xe}$ and ${ }^{127} \mathrm{I}(\mathrm{d}, 7 \mathrm{n}){ }^{122} \mathrm{Xe}$ production routes.

\section{${ }^{124} \mathrm{Xe}(p, x){ }^{122} \mathrm{Xe}$}

The two experimental datasets available in the literature are shown in Fig. 151 [311, 312] together with the TENDL calculations. These two datasets were used in the statistical fitting procedure. The data and their experimental uncertainties are shown in Fig. 152 together with the Padé fit $(L=5$, $\left.N=15, \chi^{2}=1.01\right)$ and estimated uncertainty in percentages, including $4 \%$ systematic uncertainty (right-hand scale).
Fig. 151 Two experimental datasets for the ${ }^{124} \mathrm{Xe}(\mathrm{p}, \mathrm{x})^{122} \mathrm{Xe}$ reaction available in the literature $[311,312]$, and TENDL calculations

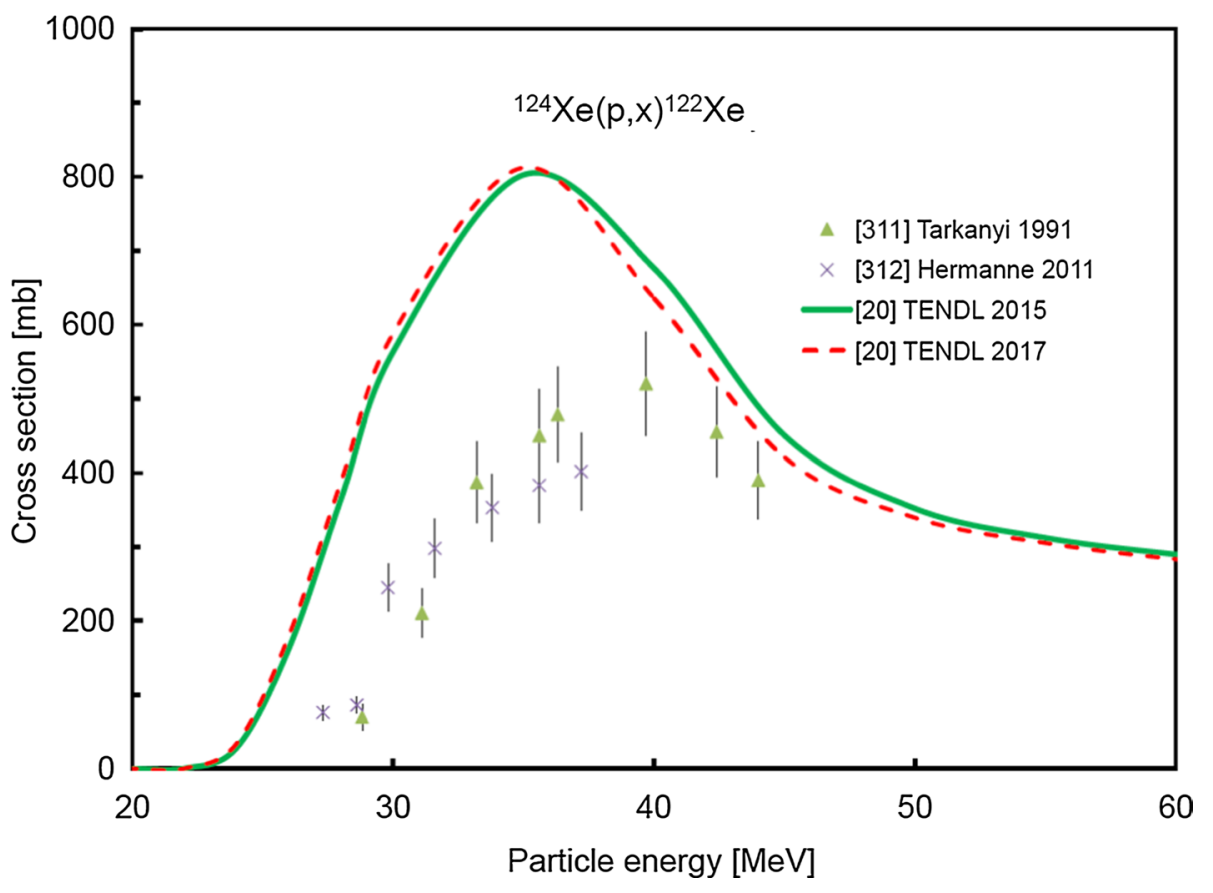


Fig. 152 Two experimental datasets for the ${ }^{124} \mathrm{Xe}(\mathrm{p}, \mathrm{x}){ }^{122} \mathrm{Xe}$ reaction $[311,312]$ with the Padé fit $(L=5, N=15$, $\chi^{2}=1.01$, solid line) and estimated total uncertainties in percentages, including $4 \%$ systematic uncertainty (dashed line, right-hand scale)

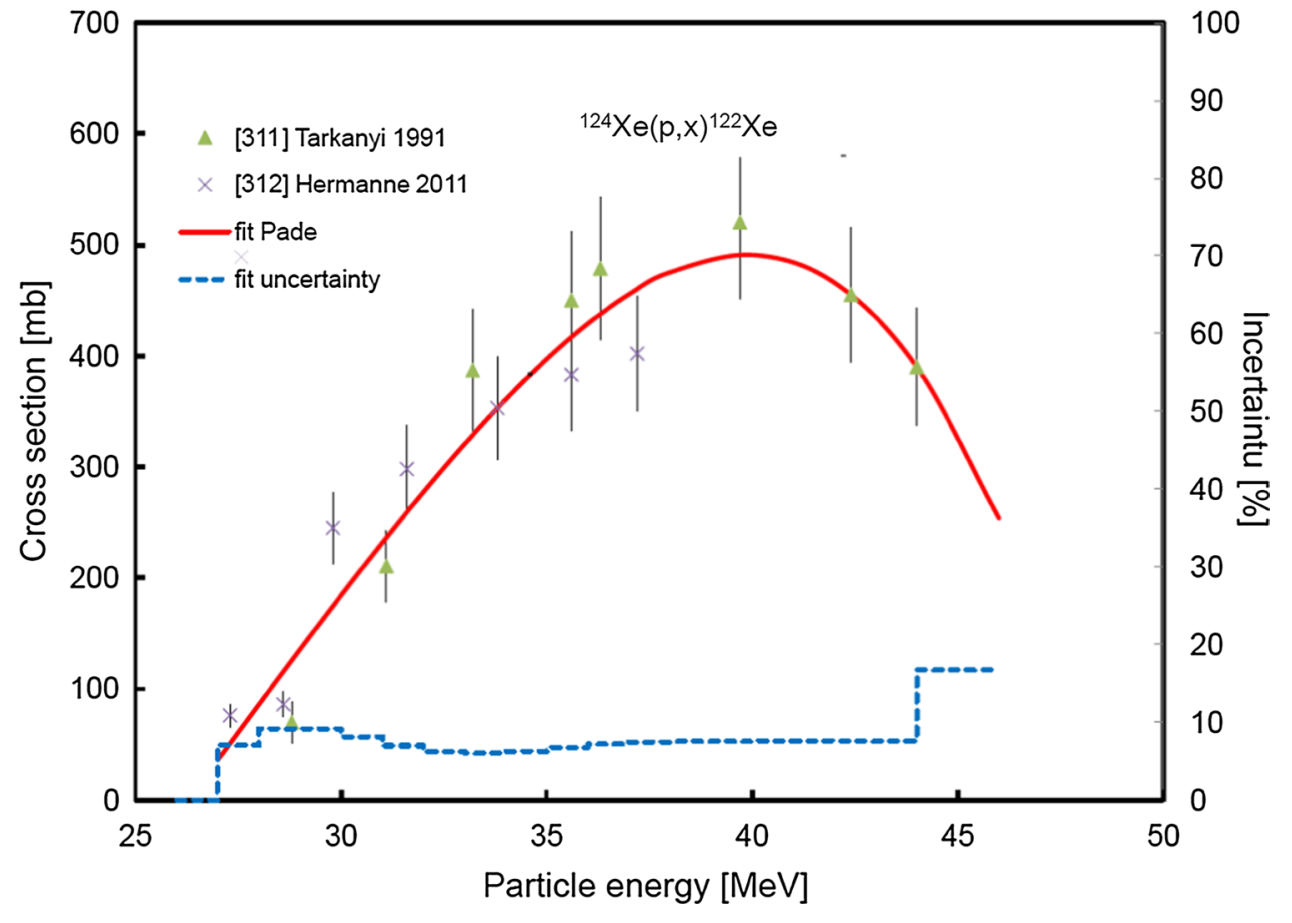




\section{${ }^{127} I(p, 6 n)^{122} X e$}

The three experimental datasets available in the literature are shown in Fig. 153 [313-315] together with the TENDL calculations. All three datasets were used in the statistical fitting procedure. These data and their experimental uncertainties are shown in Fig. 154 together with the Padé fit ( $\left.L=9, N=21, \chi^{2}=0.99\right)$ and estimated uncertainty in percentages, including $4 \%$ systematic uncertainty (right-hand scale).
Fig. 153 Three experimental datasets for the ${ }^{127} \mathrm{I}(\mathrm{p}, 6 \mathrm{n}){ }^{122} \mathrm{Xe}$ reaction available in the literature [313-315], and TENDL calculations

Fig. 154 Three experimental datasets for the ${ }^{127} \mathrm{I}(\mathrm{p}, 6 \mathrm{n})^{122} \mathrm{Xe}$ reaction [313-315] with the Padé fit $(L=7, N=21$, $\chi^{2}=1.03$, solid line) and estimated total uncertainties in percentages, including $4 \%$ systematic uncertainty (dashed line, right-hand scale)
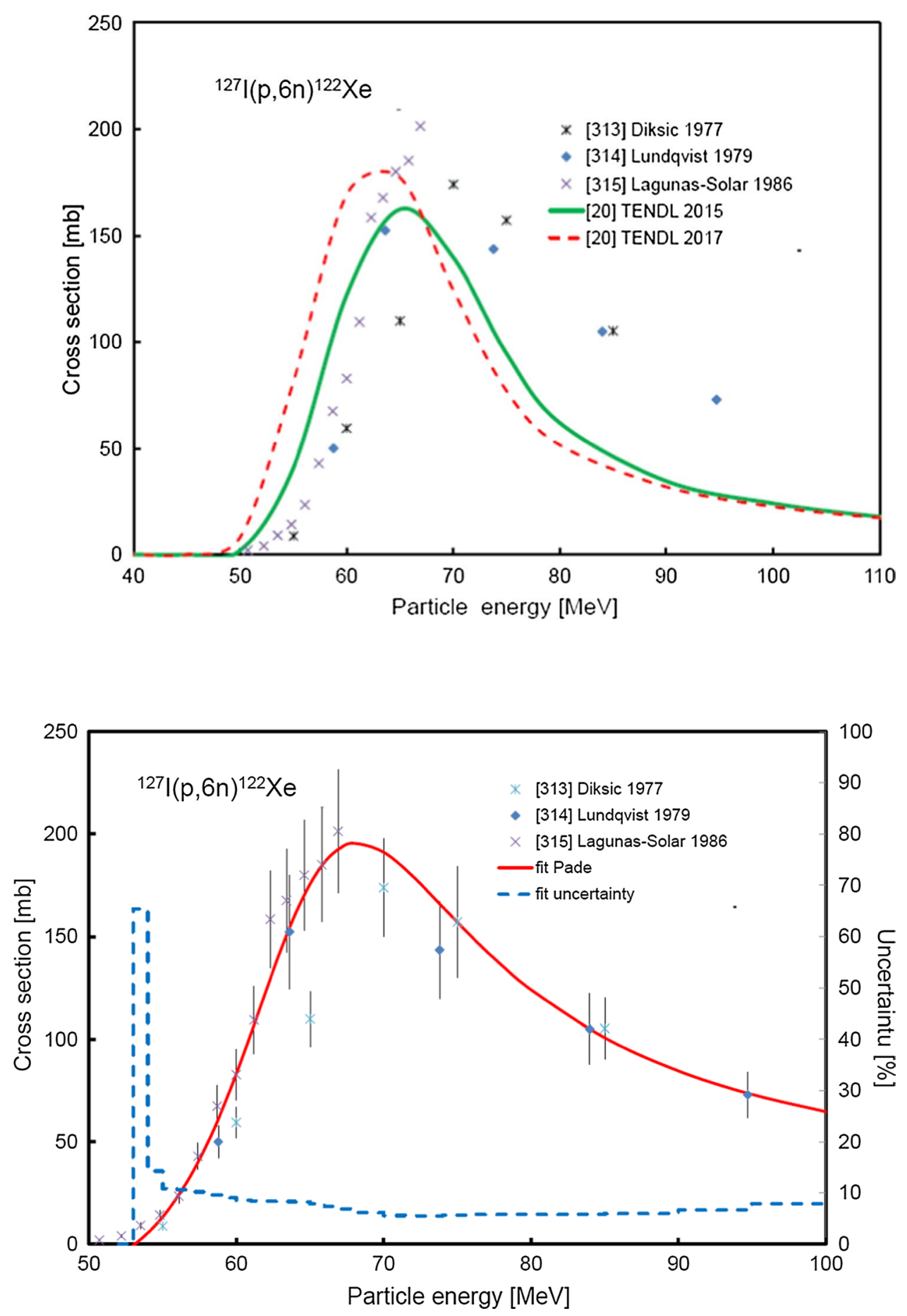
${ }^{127} I(d, 7 n){ }^{122} X e$

The two experimental datasets available in the literature are shown in Fig. 155 [316, 317] together with the TENDL calculations. After correcting the energy scale of Ref. [316], the two datasets were used in the statistical fitting procedure. The selected data and their experimental uncertainties are shown in Fig. 156 together with the Padé fit $(L=12, N=21$, $\left.\chi^{2}=1.31\right)$ and estimated uncertainty in percentages, including $4 \%$ systematic uncertainty (right-hand scale).
Fig. 155 Two experimental datasets for the ${ }^{127} \mathrm{I}(\mathrm{d}, 7 \mathrm{n})^{122} \mathrm{Xe}$ reaction available in the literature [316, 317], and TENDL calculations - with corrected energy scale for Ref. [316] data
Fig. 156 Two experimental datasets for the ${ }^{127} \mathrm{I}(\mathrm{d}, 7 \mathrm{n})^{122} \mathrm{Xe}$ reaction [316, 317] with the Padé fit $(L=12, N=21$, $\chi^{2}=1.31$, solid line) and estimated total uncertainties in percentages, including $4 \%$ systematic uncertainty (dashed line, right-hand scale)
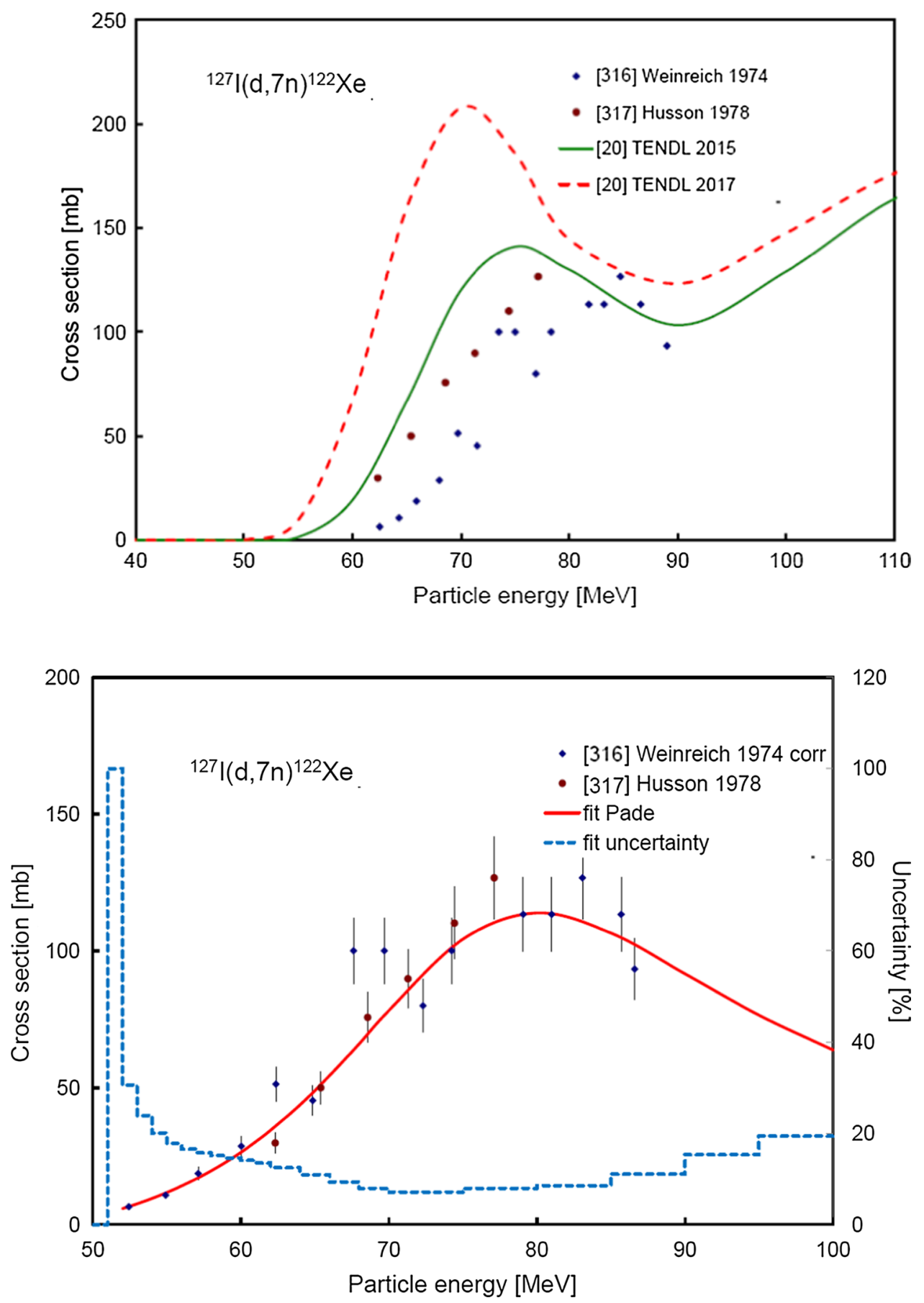
Thick target yields for production of ${ }^{122}$ Xe parent

of short-lived ${ }^{122}$ |

See Fig. 157.

Fig. 157 Thick target yields calculated from the recommended cross sections for the ${ }^{124} \mathrm{Xe}(\mathrm{p}, \mathrm{x}){ }^{122} \mathrm{Xe},{ }^{127} \mathrm{I}(\mathrm{p}, 6 \mathrm{n}){ }^{122} \mathrm{Xe}$ and ${ }^{127} \mathrm{I}(\mathrm{d}, 7 \mathrm{n})^{122} \mathrm{Xe}$ reactions

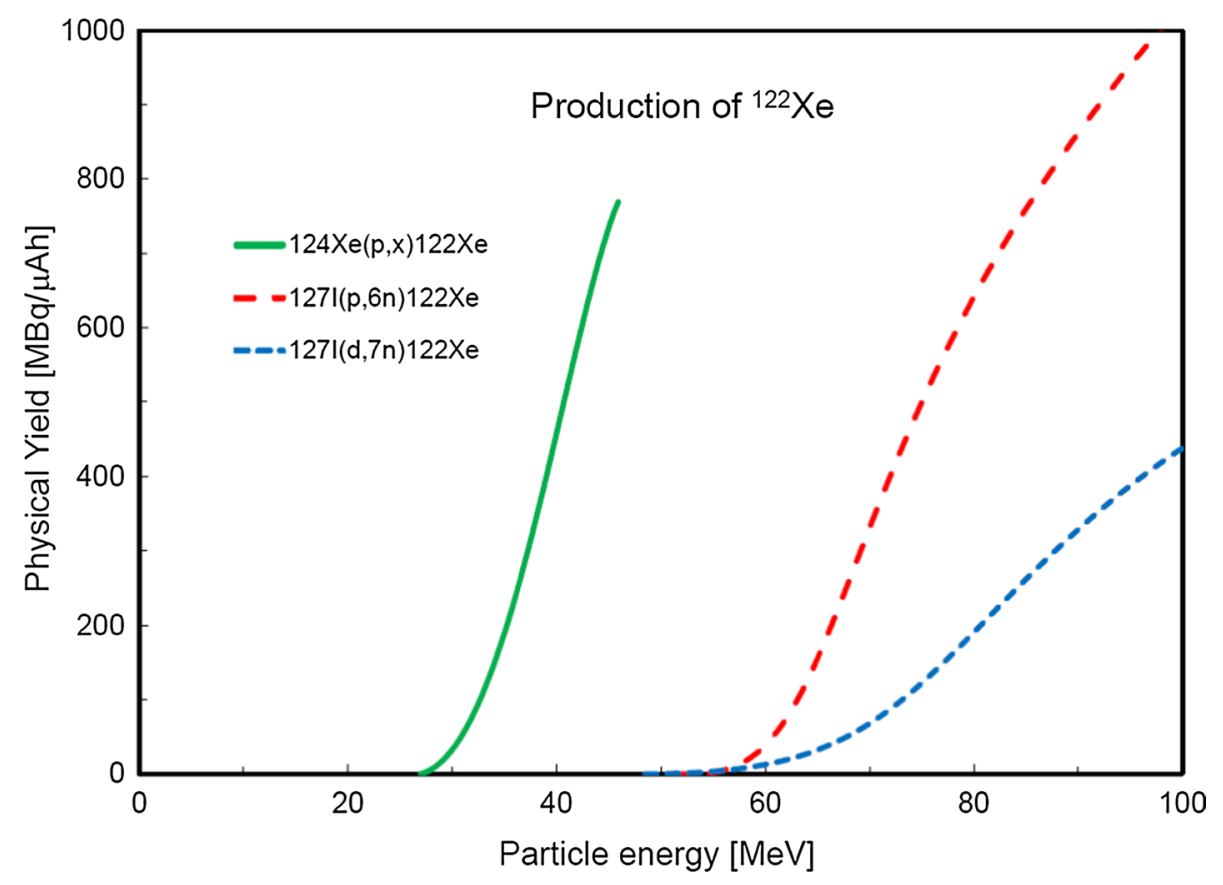


Production of ${ }^{128} \mathrm{Ba}$ parent $\left(T_{1 / 2}=2.43 \mathrm{~d}\right)$ of short-lived ${ }^{128} \mathrm{Cs}\left(T_{1 / 2}=3.66 \mathrm{~min}\right)$

Applications: Short-lived generator-produced ${ }^{128} \mathrm{Cs}$ can be used in a similar manner to ${ }^{82} \mathrm{Rb}$ for myocardial perfusion examinations.

${ }^{128} \operatorname{Cs}(3.66 \mathrm{~min}): \beta^{+}(68.8 \%)$, and $E_{\gamma}(\mathrm{keV})\left(P_{\gamma}(\%)\right): 442.901$ (26.8).

${ }^{128} \mathrm{Ba}(2.43 \mathrm{~d}): E_{\gamma}(\mathrm{keV})\left(P_{\gamma}(\%)\right): 273.44(14.5)$, and by means of radiation from daughter ${ }^{128} \mathrm{Cs}$.
Only the ${ }^{133} \mathrm{Cs}(p, 6 n){ }^{128} \mathrm{Ba}$ reaction has been evaluated.

\section{${ }^{133} \mathrm{Cs}(\mathrm{p}, 6 \mathrm{n}){ }^{128} \mathrm{Ba}$}

The four experimental datasets available in the literature are shown in Fig. 158 [318-321] together with the TENDL calculations. All four datasets were used in the statistical fitting procedure. These data and their experimental uncertainties are shown in Fig. 159 together with the Padé fit $(L=18$, $N=51, \chi^{2}=1.79$ ) and estimated uncertainty in percentages, including $4 \%$ systematic uncertainty (right-hand scale).
Fig. 158 Four experimental datasets for the ${ }^{133} \mathrm{Cs}(\mathrm{p}, 6 \mathrm{n}){ }^{128} \mathrm{Ba}$ reaction available in the literature [318-321], and TENDL calculations

Fig. 159 Four experimental datasets for the ${ }^{133} \mathrm{Cs}(\mathrm{p}, 6 \mathrm{n}){ }^{128} \mathrm{Ba}$ reaction [318-321] with the Padé fit $(L=18, N=51$, $\chi^{2}=1.79$, solid line) and estimated total uncertainties in percentages, including $4 \%$ systematic uncertainty (dashed line, right-hand scale)

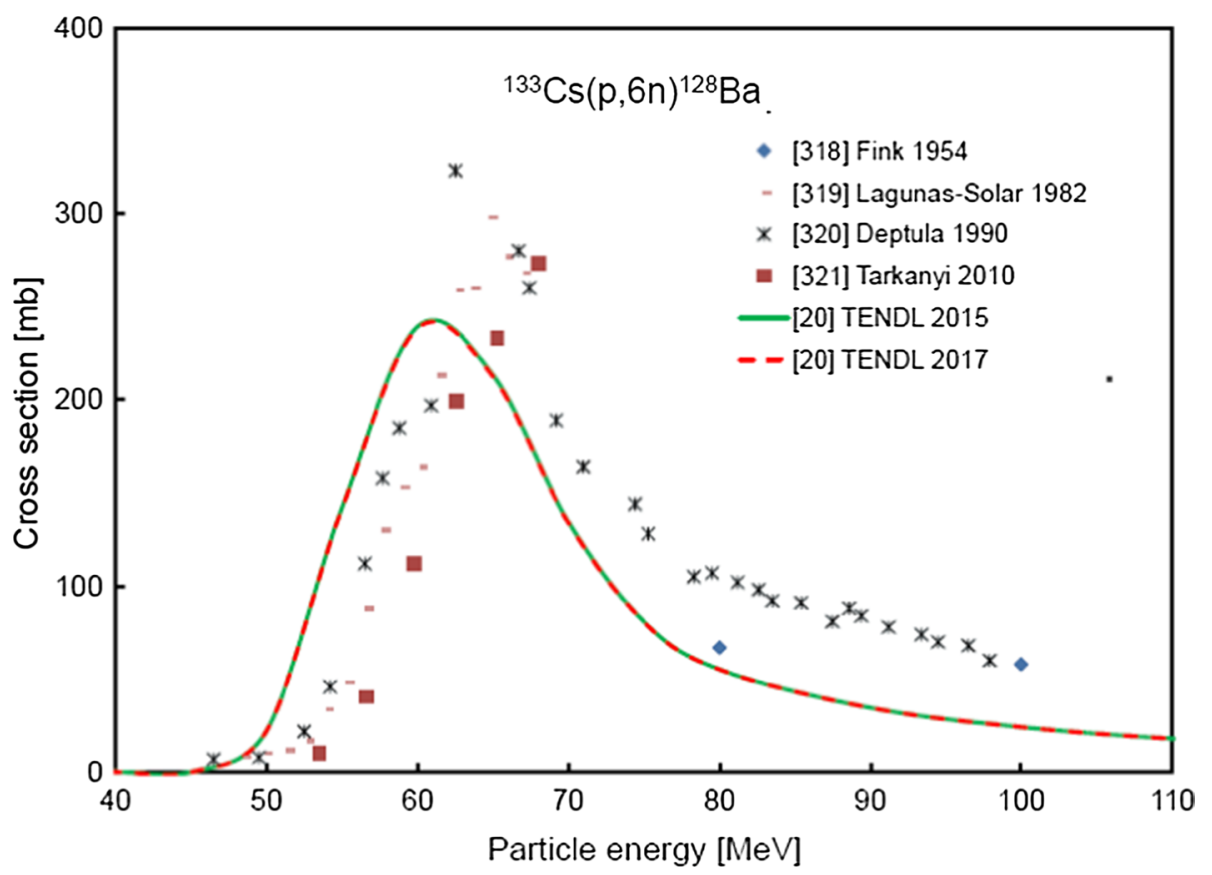


Thick target yields for ${ }^{128} \mathrm{Ba}$ parent of short-lived ${ }^{128} \mathrm{Cs}$

See Fig. 160.

Fig. 160 Thick target yields calculated from the recommended cross sections for the

${ }^{133} \mathrm{Cs}(\mathrm{p}, 6 \mathrm{n}){ }^{128} \mathrm{Ba}$ reaction

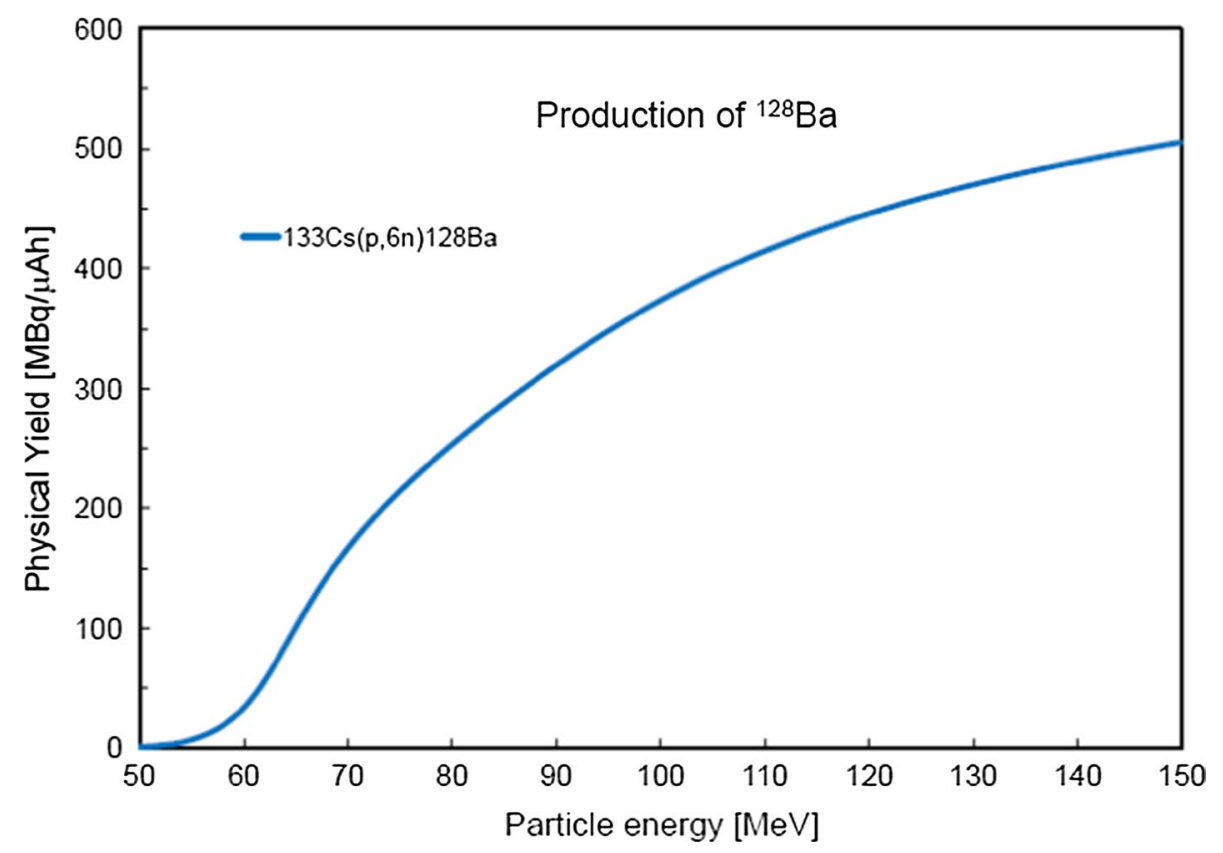


Production of ${ }^{140} \mathrm{Nd}$ parent $\left(T_{1 / 2}=3.37 \mathrm{~d}\right)$

of short-lived ${ }^{140} \operatorname{Pr}\left(T_{1 / 2}=3.39 \mathrm{~min}\right)$

Applications: EC decay of ${ }^{140} \mathrm{Nd}\left(\mathrm{EC}=100 \%, T_{1 / 2}=3.37\right.$ d) produces short-lived ${ }^{140} \operatorname{Pr}\left(T_{1 / 2}=3.39 \mathrm{~min}, \beta^{+}=51.0 \%\right.$, $\left.E_{\beta+}(\max )=2.366 \mathrm{MeV}\right)$, which undergoes $\mathrm{EC} / \beta^{+}$decay to stable ${ }^{140} \mathrm{Ce}$. Parent-daughter ${ }^{140} \mathrm{Nd} /{ }^{140} \mathrm{Pr}$ has been proposed as a radionuclide generator, or as an in vivo generator system for PET studies.

${ }^{140} \operatorname{Pr}(3.39 \mathrm{~min}): \beta^{+}(51.0 \%)$, and $E_{\gamma}(\mathrm{keV})\left(P_{\gamma}(\%)\right): 306.9$ (0.147), 1596.1 (0.49).

${ }^{140} N d(3.37 d)$ : detected by means of radiation emitted by daughter ${ }^{140} \mathrm{Pr}$.
Evaluations have been undertaken of the ${ }^{141} \operatorname{Pr}(\mathrm{p}, 2 \mathrm{n}){ }^{140} \mathrm{Nd}$, ${ }^{141} \mathrm{Pr}(\mathrm{d}, 3 \mathrm{n}){ }^{140} \mathrm{Nd}$ and ${ }^{\mathrm{nat}} \mathrm{Ce}\left({ }^{3} \mathrm{He}, \mathrm{xn}\right){ }^{140} \mathrm{Nd}$ production routes.

${ }^{141} \operatorname{Pr}(p, 2 n){ }^{140} \mathrm{Nd}$

The three experimental datasets available in the literature are shown in Fig. 161 [322-324] together with the TENDL calculations. One dataset was rejected (Hogan [322], values too high), and the remaining two sets were used in the statistical fitting procedure. The selected data and their experimental uncertainties are shown in Fig. 162 together with the Padé fit ( $\left.L=10, N=121, \chi^{2}=0.78\right)$ and estimated uncertainty in percentages, including $4 \%$ systematic uncertainty (righthand scale).
Fig. 161 Three experimental datasets for the ${ }^{141} \operatorname{Pr}(\mathrm{p}, 2 \mathrm{n}){ }^{140} \mathrm{Nd}$ reaction available in the literature [322-324], and TENDL calculations

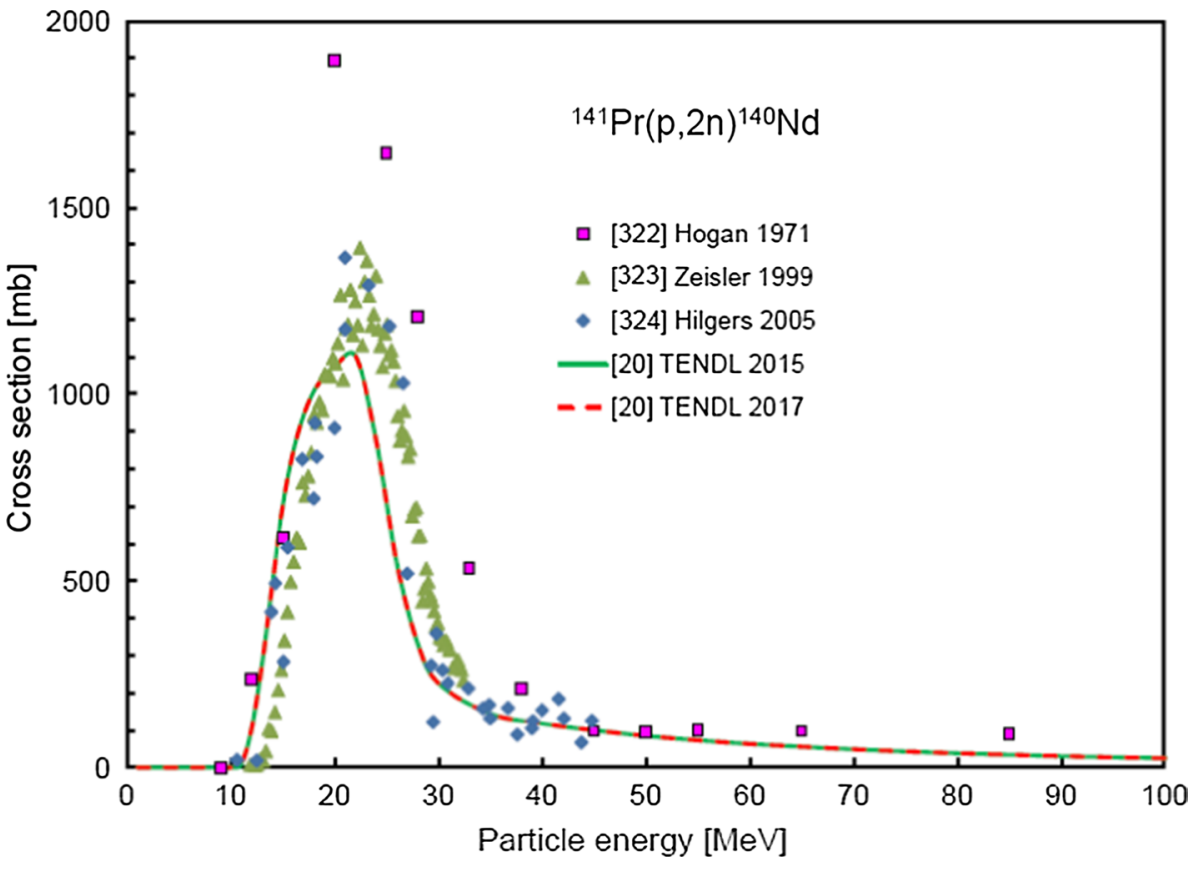


Fig. 162 Two selected experimental datasets for the ${ }^{141} \operatorname{Pr}(\mathrm{p}, 2 \mathrm{n}){ }^{140} \mathrm{Nd}$ reaction $[323$ 324] with the Padé fit ( $L=10$, $N=121, \chi^{2}=0.78$, solid line) and estimated total uncertainties in percentages, including $4 \%$ systematic uncertainty (dashed line, right-hand scale)

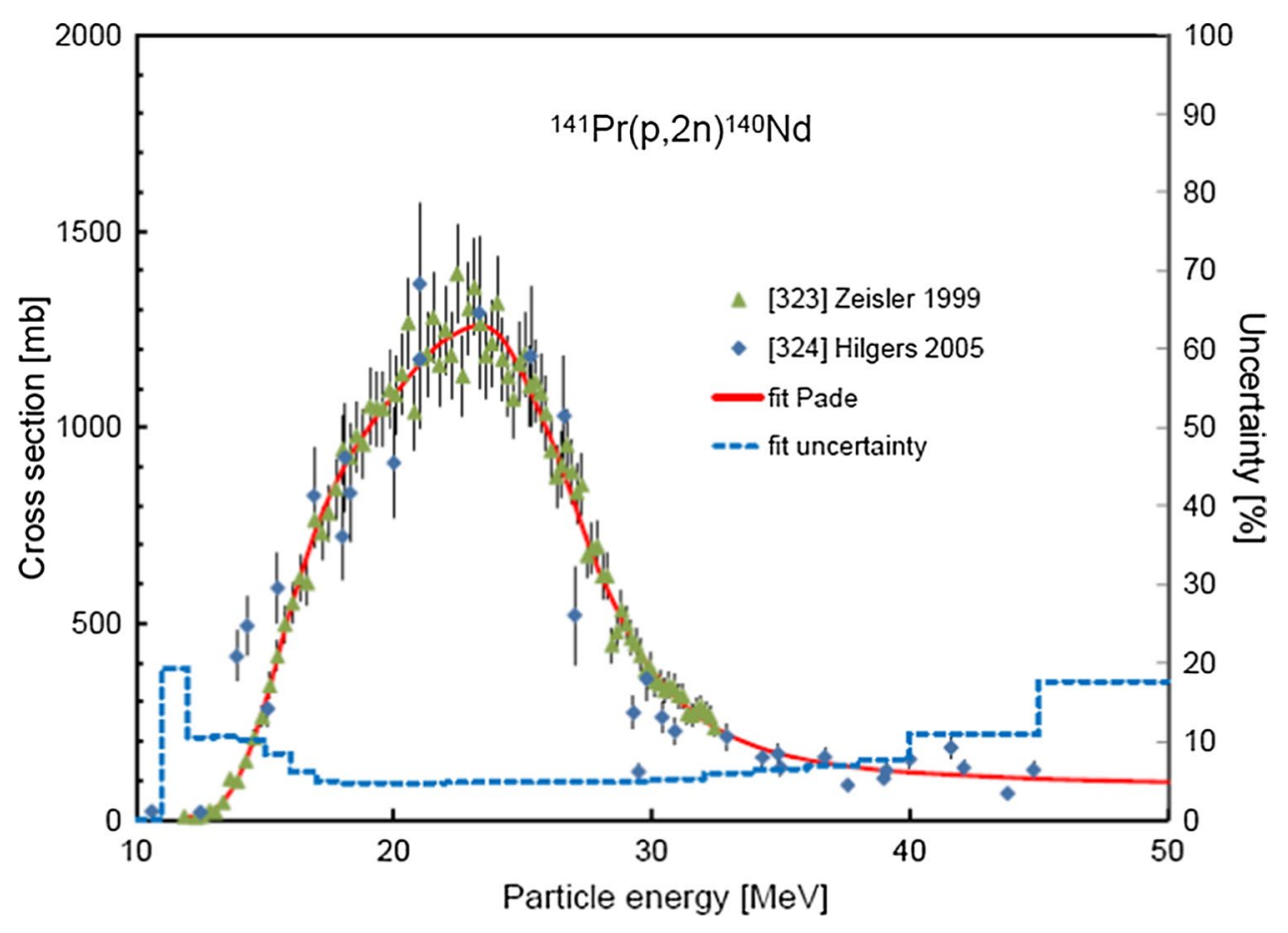


${ }^{141} \operatorname{Pr}(\mathrm{d}, 3 \mathrm{n}){ }^{140} \mathrm{Nd}$

The two experimental datasets available in the literature are shown in Fig. 163 [325, 326] together with the TENDL calculations. Both datasets were used in the statistical fitting procedure, as shown in Fig. 164 together with the Padé fit ( $\left.L=9, N=17, \chi^{2}=0.466\right)$ and estimated uncertainty in percentages, including $4 \%$ systematic uncertainty (right-hand scale).
Fig. 163 Two experimental datasets for the ${ }^{141} \operatorname{Pr}(\mathrm{d}, 3 \mathrm{n}){ }^{140} \mathrm{Nd}$ reaction available in the literature $[325,326]$, and TENDL calculations
Fig. 164 Two experimental datasets for the ${ }^{141} \operatorname{Pr}(\mathrm{d}, 3 \mathrm{n})^{140} \mathrm{Nd}$ reaction $[325,326]$ with the Padé fit $(L=9, N=17$, $\chi^{2}=0.466$, solid line) and estimated total uncertainties in percentages, including $4 \%$ systematic uncertainty (dashed line, right-hand scale)
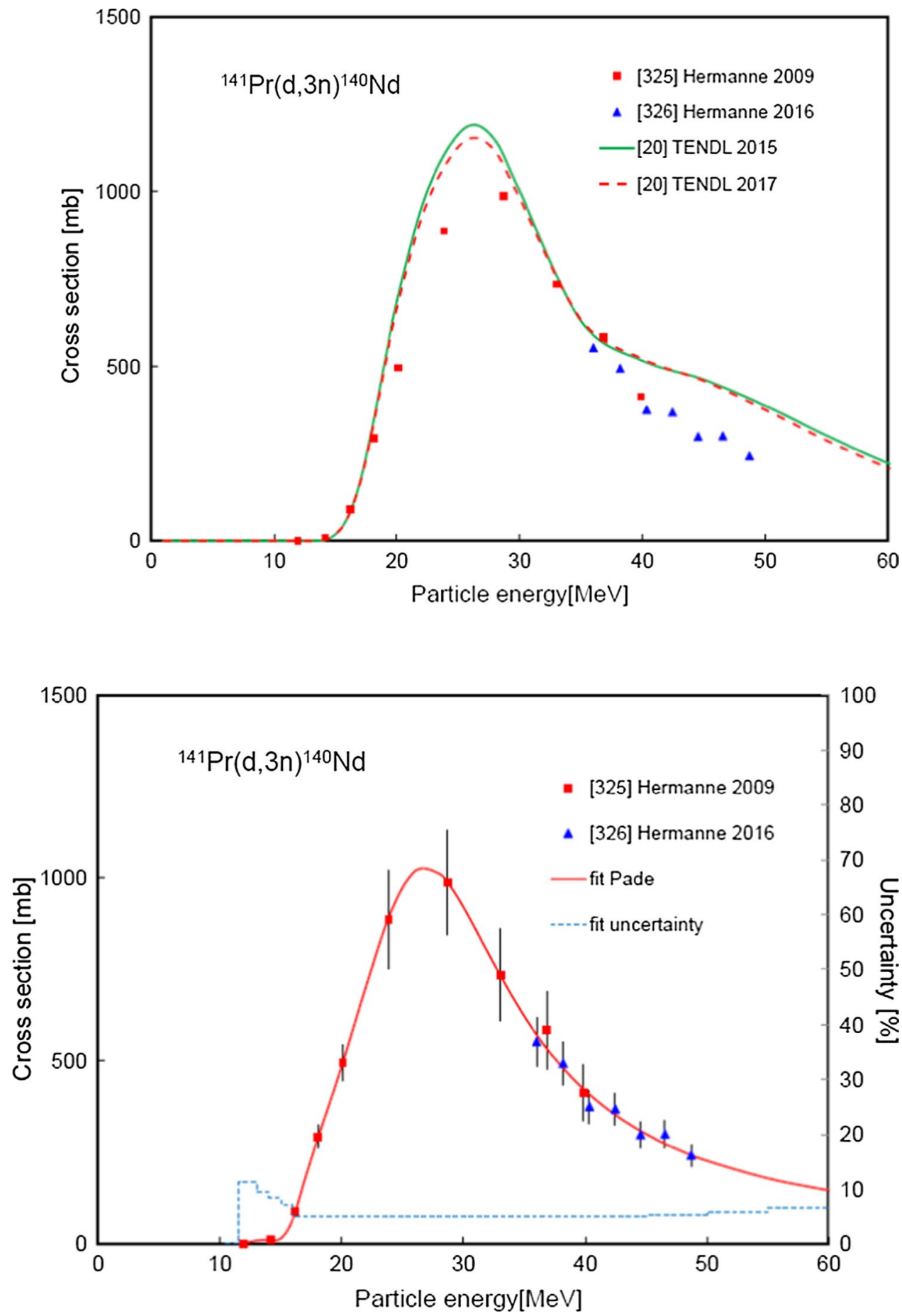


\section{${ }^{\text {nat }} \mathrm{Ce}\left({ }^{3} \mathrm{He}, \mathrm{xn}\right){ }^{140} \mathrm{Nd}$}

Only a single experimental dataset has been found in the literature [324], as illustrated in Fig. 165 together with the TENDL calculations. ${ }^{3} \mathrm{He}$ beams are rarely available for use, and therefore TENDL evaluators have not attempted to improve the description of this complicated channel, in which break-up and transfer effects may dramatically change the shape and magnitude of the calculated cross sections. This dataset and associated experimental uncertainties were adopted in the statistical fitting procedure. The results are shown in Fig. 166 together with the Padé fit $(L=9, N=18$, $\chi^{2}=1.59$, solid line) and estimated uncertainty in percentages, including $4 \%$ systematic uncertainty (right-hand scale).
Fig. 165 One experimental dataset for the ${ }^{\text {nat }} \mathrm{Ce}\left({ }^{3} \mathrm{He}, \mathrm{xn}\right){ }^{140} \mathrm{Nd}$ reaction available in the literature [324], and TENDL calculations
Fig. 166 One experimental dataset for the ${ }^{\text {nat }} \mathrm{Ce}\left({ }^{3} \mathrm{He}, \mathrm{xn}\right){ }^{140} \mathrm{Nd}$ reaction [324] with the Padé fit ( $L=9$, $N=18, \chi^{2}=1.59$, solid line) and estimated total uncertainties in percentages, including $4 \%$ systematic uncertainty (dashed line, right-hand scale)
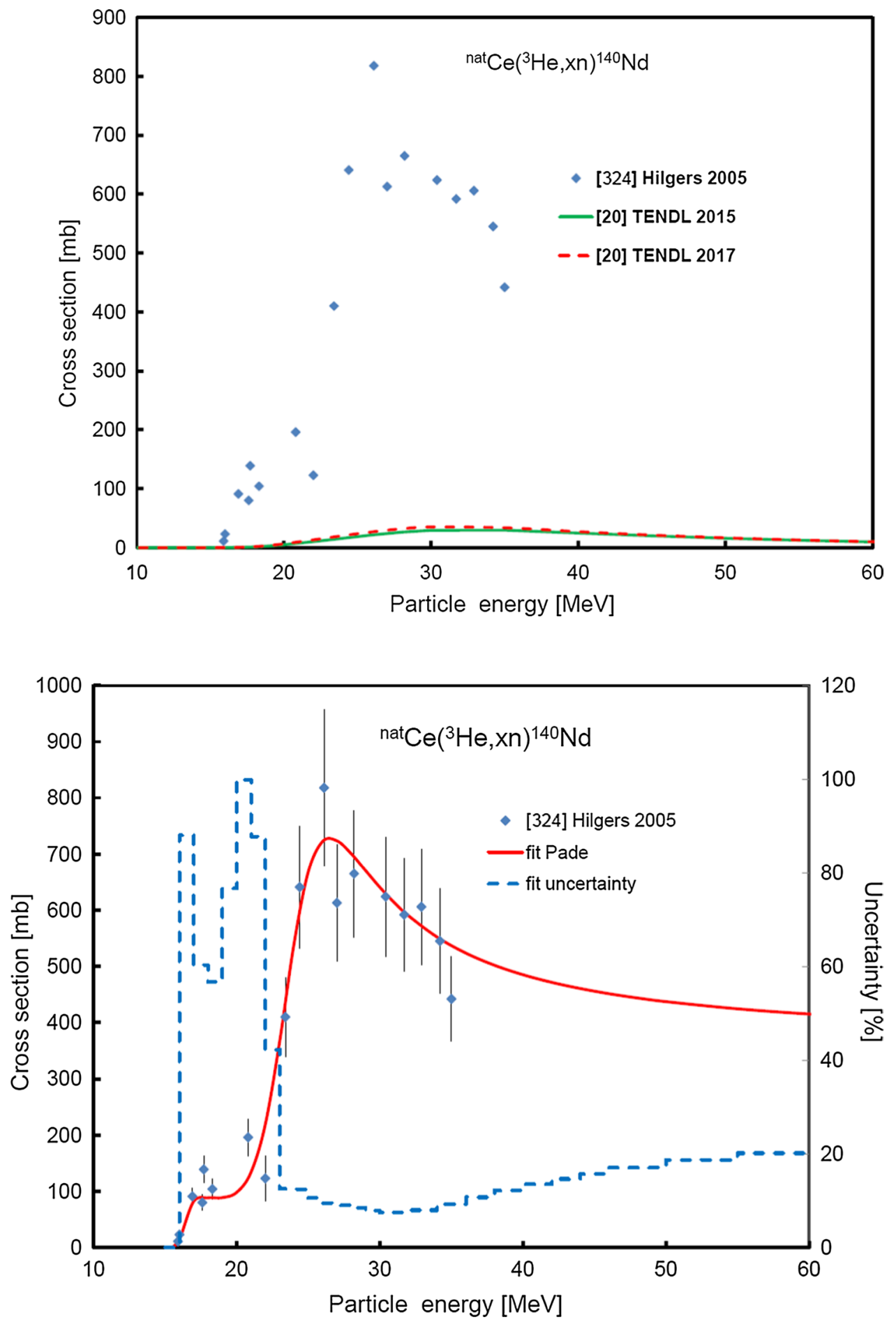
Thick target yields for production of ${ }^{140} \mathrm{Nd}$ parent of short-lived ${ }^{140} \mathrm{Pr}$

See Fig. 167.

Fig. 167 Thick target yields calculated from the recommended cross sections for the ${ }^{141} \operatorname{Pr}(\mathrm{p}, 2 \mathrm{n}){ }^{140} \mathrm{Nd}$,

${ }^{141} \operatorname{Pr}(\mathrm{d}, 3 \mathrm{n}){ }^{140} \mathrm{Nd}$ and

${ }^{\text {nat }} \mathrm{Ce}\left({ }^{3} \mathrm{He}, \mathrm{xn}\right){ }^{140} \mathrm{Nd}$ reactions

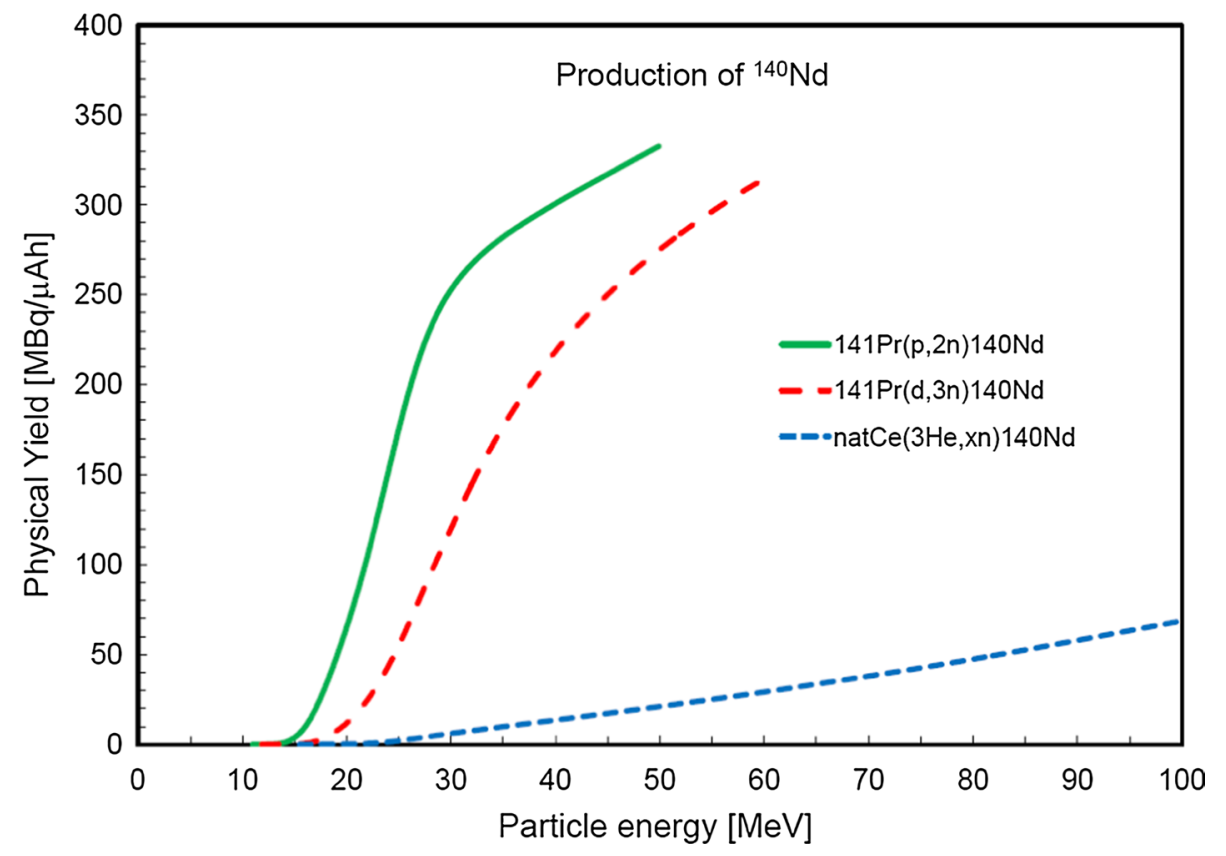




\section{Evaluated excitation functions: an overall assessment}

A significant amount of effort has been expended to assemble, evaluate, analyse and recommend suitable cross-section datasets to ensure that a series of radionuclides may be generated with acceptable purity in an optimum manner for PET imaging. Obviously, confidence in such recommended data is highly dependent on their quality and validity as addressed and improved by in-depth evaluations. As derived, these data can also be assessed to identify those cross sections and their resulting excitation functions that can be improved further by means of additional measurements. Additionally, integral measurements (production thick target yields) are very important and need to be performed in order to assist in the benchmaring and validation of the recommended data.

Improved radionuclidic purity may be an issue that emerges from research-based applications in nuclear medicine. Under such circumstances, excitation functions for these radionuclidic impurities are also required to aid in the optimisation of target composition and energy range of the beam within the target in order to avoid or at least minimise their production. These data requirements have not been addressed in the current studies, but remain an important part of what would normally constitute an evolutionary programme of work that may hopefully lead up to regular medical application.

Some critical comments can be justifiably applied as to the quality of any evaluated cross-section data, particularly when the quoted uncertainties of such recommended excitation functions do not reflect the complexity of various underlying and often ill-defined factors. For example, difficulties arise when attempting to judge the quality of experimental data on the basis of only the original publication(s), as significant details on the measurement and associated data evaluations are required for such an exercise but are often omitted from journal papers. Reported uncertainties do not exceed seven or eight percent in many cases for very different types of reaction and measurement methodology that makes such modest percentages effectively unrealistic. Some laboratories are known to possess greater expertise than others, and/or have a better technical background in cross-section studies. Such personnel and facilities most frequently generate more reliable experimental data that implies greater weight should be applied to their datasets in the evaluation process. However, under such circumstances, less reliable data and their questionable uncertainties are considered in many evaluations with the same weighting, and so distort the final recommended values. The thoroughness of compilation, systematic application of all known corrections, and strictness in data selection also depend strongly on the subjective knowledge and analytical behaviour of individual evaluators. This results in a quality difference between the recommendations of evaluators that is not reflected in the uncertainties of their final sets of recommended nuclear data.

Comprehensive comparisons have been made of the measured cross-section results of the 69 reactions studied in this work with the equivalent evolving predictions of the two TENDL libraries based on theoretical cross-section calculations of the TALYS code system. No important changes would appear to have been introduced into the code between 2015 and 2017 that affect these particular reactions because, in the majority of cases, the results for TENDL-2017 are identical to or only marginally differ from the contents of the TENDL-2015 database. A rather surprising observation is that, where large differences exist, the 2017 database exhibits larger disagreements with the experimental results than the 2015 edition (see for example, Figs. 120 and 155). However, the readily available on-line predictions are in acceptable agreement with the overall shape of the measured and evaluated excitation functions, and are therefore useful for the estimation of other unmeasured nuclear processes. The main shortcomings observed in the TENDL libraries and related TALYS reaction modelling can be identified with the following behaviour:

1. Energy shift of high energy reactions with the emission of multiple particles.

2. Underestimation of the production of some isomeric states which is reflected in the underestimation of cumulative processes.

3. Poor quantification of the magnitude of alpha-particle induced reactions near their maximum and at higher energies.

4. Underestimation of the cross section for a single ${ }^{3} \mathrm{He}-$ induced reaction.

5. Underestimation of the cross section for deuteron induced reactions, especially from the threshold to the maximum of the excitation function.

6. Unexplained strange shapes within some $(\mathrm{p}, \mathrm{n})$ reactions (e.g., plateau near maximum).

Many of these shortcomings are related to known problems in the theoretical modelling that will be shared by results calculated using different reaction codes.

Additional improved experimental studies of particular production routes are clearly merited, as defined by the nature of some of the existing cross-section data to be found throughout Section Results for charged-particle reactions. However, there are also other reactions that require consideration and analyses of the form undertaken above [327, 328], along with the need for better quantified studies of specific positron and X-ray emission probabilities [329]. 
New measurements and evaluations are required of the activation cross sections for proton-induced reactions with energies up to $250 \mathrm{MeV}:{ }^{11} \mathrm{C},{ }^{13} \mathrm{~N},{ }^{14,15} \mathrm{O},{ }^{30} \mathrm{P}$ and ${ }^{38} \mathrm{~K}$. More extensive cross-section studies would also be beneficial to achieve the optimum production of ${ }^{34 \mathrm{~m}} \mathrm{Cl},{ }^{43} \mathrm{Sc},{ }^{45} \mathrm{Ti},{ }^{48} \mathrm{~V}$, ${ }^{49} \mathrm{Cr},{ }^{51} \mathrm{Mn},{ }^{57} \mathrm{Ni},{ }^{75} \mathrm{Br},{ }^{77} \mathrm{Kr},{ }^{81} \mathrm{Rb},{ }^{83} \mathrm{Sr},{ }^{95} \mathrm{Ru},{ }^{121} \mathrm{I}$ and ${ }^{152} \mathrm{~Tb}$. Improved decay data are identified with the need for accurate absolute positron and X-ray emission probabilities for ${ }^{57} \mathrm{Ni}$, ${ }^{72} \mathrm{As},{ }^{73} \mathrm{Se},{ }^{75,76} \mathrm{Br},{ }^{77} \mathrm{Kr},{ }^{81,82 \mathrm{~m}} \mathrm{Rb},{ }^{83} \mathrm{Sr},{ }^{86} \mathrm{Y},{ }^{89} \mathrm{Zr},{ }^{94 \mathrm{~m}} \mathrm{Tc}$ and ${ }^{120} \mathrm{I}$. On balance, reviews of the nuclear data requirements in nuclear medicine would appear to be appropriate approximately every 10 years to ensure a continued and well-defined international focus on ensuring that the necessary technical information is immediately to hand when required.

\section{Conclusions}

Substantial extensions and significant improvements have been made to the IAEA-NDS recommended cross-section database for the production of PET radionuclides. Evaluations were performed on 69 reactions for direct, indirect or generator production of ${ }^{44} \mathrm{Sc},{ }^{44} \mathrm{Ti},{ }^{52} \mathrm{Mn},{ }^{52 \mathrm{~m}} \mathrm{Mn},{ }^{52} \mathrm{Fe}$, ${ }^{55} \mathrm{Co},{ }^{61} \mathrm{Cu},{ }^{62} \mathrm{Cu},{ }^{62} \mathrm{Zn},{ }^{66} \mathrm{Ga},{ }^{68} \mathrm{Ga},{ }^{68} \mathrm{Ge},{ }^{72} \mathrm{As},{ }^{72} \mathrm{Se},{ }^{73} \mathrm{Se}$, ${ }^{76} \mathrm{Br},{ }^{82} \mathrm{Rb},{ }^{82 \mathrm{~m}} \mathrm{Rb},{ }^{82} \mathrm{Sr},{ }^{86} \mathrm{Y},{ }^{89} \mathrm{Zr},{ }^{90} \mathrm{Nb},{ }^{94 \mathrm{~m}} \mathrm{Tc},{ }^{110 \mathrm{~m}} \mathrm{In},{ }^{110} \mathrm{Sn}$, ${ }^{118} \mathrm{Sb},{ }^{118} \mathrm{Te},{ }^{120} \mathrm{I},{ }^{122} \mathrm{I},{ }^{122} \mathrm{Xe},{ }^{128} \mathrm{Cs},{ }^{128} \mathrm{Ba},{ }^{140} \mathrm{Pr}$ and ${ }^{140} \mathrm{Nd}$. A Padé fitting method was applied to the evaluated datasets selected, and uncertainties for all of the recommended data were deduced. The experimental data were compared with theoretical predictions taken from both the TENDL-2015 and TENDL-2017 libraries, sometimes exhibiting significant disagreements in the magnitude and shape of the resulting excitation functions.

As well as a lack of published data for specific reactions, significant disagreements were also found to exist between various equivalent experimental data. All of the recommended cross-section data were used to derive integral or production thick target yields for direct practical application.

All of the numerical reference cross-section data with their corresponding uncertainties and deduced integral thick target yields are available on-line at the IAEA-NDS medical portal www-nds.iaea.org/medportal/ [2] and also at the IAEA-NDS web page www-nds.iaea.org/medical/posit ron_emitters.html.

These evaluated experimental data are important for existing and potential applications in nuclear medicine, and may also have useful roles in other fields of non-energy related nuclear studies.

Acknowledgements Our sincere thanks are extended to all colleagues who have contributed to and worked on this project over the previous 5 years. The studies undertaken and the preparation of this paper would not have been possible without the full support, hard work and efforts of a large number of individuals and institutions. The IAEA is grateful to all participant laboratories for their assistance in the work and support of the CRP meetings and activities. Work described in this paper would not have been possible without IAEA Member State contributions. Studies at ANL were supported by the U.S. Department of Energy, Office of Science, Office of Nuclear Physics, under contract no. DE-AC-06CH11357.

Open Access This article is distributed under the terms of the Creative Commons Attribution 4.0 International License (http://creativeco mmons.org/licenses/by/4.0/), which permits unrestricted use, distribution, and reproduction in any medium, provided you give appropriate credit to the original author(s) and the source, provide a link to the Creative Commons license, and indicate if changes were made.

\section{References}

1. Gul K, Hermanne A, Mustafa MG, Nortier FM, Obložinský P, Qaim SM, Scholten B, Shubin Yu, Takács S, Tárkányi FT, Zhuang Y Charged particle cross-section database for medical radioisotope production: diagnostic radioisotopes and monitor reactions, IAEA report IAEA-TECDOC-1211, May 2001, IAEA, Vienna, Austria. Available online at: www-nds.iaea.org/publi cations/tecdocs/iaea-tecdoc-1211.pdf. Monitor data are available online at: www-nds.iaea.org/medical/medical-old/monitor_react ions.html. Medical isotope production data are online at: wwwnds.iaea.org/medicalportal/

2. IAEA medical portal - charged particle cross section database for medical radioisotope production, Updated 2003-2004, and beyond. Available online at: www-nds.iaea.org/medportal/

3. Nichols AL, Capote R (2012) Summary report of first research coordination meeting on nuclear data for charged-particle monitor reactions and medical isotope production, 3-7 December 2012, IAEA report INDC(NDS)-0630, February 2013, IAEA, Vienna, Austria. Available online at: www-nds.iaea.org/publi cations/indc/indc-nds-0630.pdf

4. NuDat, US National Nuclear Data Center, Brookhaven National Laboratory, USA. Decay data retrieval code available online at: www.nndc.bnl.gov/nudat2/

5. ENSDF: Evaluated Nuclear Structure Data File. Available online at: www.nndc.bnl.gov/ensdf/ Developed and maintained by the International Network of Nuclear Structure and Decay Data Evaluators (NSDD) (see www-nds.iaea.org/nsdd/)

6. Bĕták E, Caldeira AD, Capote R, Carlson BV, Choi HD, Guimarães FB, Ignatyuk AV, Kim SK, Király B, Kovalev SF, Menapace E, Nichols AL, Nortier M, Pompeia P, Qaim SM, Scholten B, Shubin Yu N, Sublet J-Ch, Tárkányi F (2011) Nuclear data for the production of therapeutic radionuclides. In: Qaim SM, Tárkányi F, Capote R (Technical editors) IAEA Technical Reports Series no. 473, IAEA scientific and technical report STI/DOC/010/473, IAEA, Vienna, Austria (2011) ISBN 978-92-0-115010-3. Available online at: www-nds.iaea.org/publications/tecdocs/technicalreports-series-473.pdf Data also available on-line at: www-nds. iaea.org/medportal/

7. Otuka N, Dupont E, Semkova V, Pritychenko B, Blokhin AI, Aikawa M, Babykina S, Bossant M, Cheng G, Dunaeva S, Forrest RA, Fukahori T, Furutachi N, Ganesan S, Geg Z, Gritzay OO, Herman M, Hlaváč S, Kato K, Lalremruata B, Lee YO, Makinaga A, Matsumoto K, Mikhaylyukova M, Pikulina G, Pronyaev VG, Saxena A, Schwerer O, Simakov SP, Sopper N, Suzuki R, Takács S, Tao X, Taova S, Tárkányi F, Varlamov VV, Wang J, Yang SC, Zerkin V, Zhuang Y (2014) Towards a more complete and accurate experimental nuclear reaction data library 
(EXFOR): international collaboration between nuclear reaction data centres. Nucl Data Sheets 120:272-276

8. International Nuclear Information System (INIS), IAEA, Austria, Vienna. www.iaea.org/resources/databases/inis

9. ENDF: International Evaluated Nuclear Data Files. For example: Chadwick MB, Herman M, Obložinský P, et al (2011) ENDF/B-VII.1 nuclear data for science and technology: Cross sections, covariances, fission product yields and decay data. Nucl Data Sheets 112:2887-2996; Koning AJ, Forrest RA, Kellett MA, Mills RW, Henriksson H, Rugama Y (2006) The JEFF3.1 Nuclear Data Library, JEFF report 21. NEA-OECD, Paris, France

10. Burrows TW, Dempsey P (1980) The bibliography of the integral charged particle nuclear data, 4th edn, Suppl. 1-2. BNL report BNL-NCS-50640, Brookhaven, Upton, NY, USA

11. Holden NE, Ravamataram S, Dunford CL (1985, 1986, 1987, 1988, 1989) Integral charged particle nuclear data bibliography, 1st edn, Suppl. 1-5. BNL report BNL-NCS-51771, Brookhaven, Upton, NY, USA

12. Karlstrom I, Christman DR (1975, 1978, 1983) Accelerator produced nuclides for use in biology and medicine. BNL report BNL-50448, vol 1-3, Brookhaven, Upton, NY, USA

13. Dmitriev PP (1986) Radionuclide yield in reactions with protons, deuterons, alpha particles and helium-3. IAEA report INDC(CCP)-263, IAEA, Vienna, Austria. Available online at: https://www-nds.iaea.org/publications/indc/indc-ccp-0263.pdf

14. Gandarias-Cruz D, Okamoto K (1988) Status on compilation of nuclear data for medical radioisotopes produced by accelerators. IAEA report INDC(NDS)-209, IAEA, Vienna, Austria. Available online at: https://www-nds.iaea.org/publications/indc/indcnds-0209.pdf

15. Landolt Börnstein Series Keller KA, Lange J, Münzel H, Pfenning G (1973) Excitation functions for charged-particle induced nuclear reactions. Landolt-Börnstein Series, Group I, vol 5/b, Springer, Berlin

16. Landolt Börnstein New Series. Iljinov AS, Semenov VG, Semenova MP, Sobolevsky NM, Udovenko LV. Production of radionuclides at intermediate energies, Landolt-Börnstein, New Series, Group I, vol. 13, Springer, Berlin-Heidelberg-New York-Subvolume A, 1991: Interactions of protons with targets from He to $\mathrm{Br}$-Sub-volume B, 1992: Interactions of protons with targets from $\mathrm{Kr}$ to $\mathrm{Te}-$ Sub-volume C, 1993: Interactions of protons with targets from I to Am-Sub-volume D, 1994: Interactions of protons with nuclei (supplement to I/13A, B, C). Semenov VG, Semenova MP, Sobolevsky NM, Interaction of deuterons, tritons and ${ }^{3} \mathrm{He}$-nuclei with nuclei in production of radionuclides at intermediate energies, Landolt-Börnstein New Series, Group I, vol 13F, Springer-Verlag, Berlin, Germany (1995). Semenov VG, Semenova MP, Sobolevsky NM, Interaction of particles with targets from $\mathrm{He}$ to $\mathrm{Rb}$ in production of radionuclides at intermediate energies, Landolt-Börnstein New Series, Group I, vol 13G, Springer-Verlag, Berlin, Germany (1996). Semenov VG, Semenova MP, Sobolevsky NM, Interaction of particles with targets in production of radionuclides at intermediate energies, Landolt-Börnstein New Series, Group I, vol 13H, Springer-Verlag, Berlin, Germany (1996)

17. Tobailem J, de Lassus St-Genies CH, Leveque L (1971) Sections Efficaces des Réactions Nucléaires induites par Protons, Deuterons, Particules Alpha, I. Réactions Nucléaires Moniteurs, CEA note CEA-N-1466(1) CEA, France. Tobaliem J, de Lassus St.-Genies CH (1971) Sections Efficaces des Réactions Nucléaires induites par Protons, Deuterons, Particules Alpha, CEA note CEA-N-1466(1) CEA, France

18. Albert P, Blondiaux G, Debrun JL, Giovagnoli A, Valladon M (1987) Handbook on nuclear activation data, 3-2. Activation cross-sections for elements from lithium to sulphur, IAEA
Technical Reports Series no. 273: 479-535, IAEA, Vienna, Austria. 3-3. Thick target yields for the production of radioisotopes, IAEA Technical Reports Series no. 273:537-628. IAEA, Vienna, Austria. Available online at: www-nds.iaea.org/publications/ tecdocs/technical-reports-series-273.pdf

19. Münzel H, Klewe-Nebenius H, Lange J, Pfennig G, Hemberle K (1982) Karlsruhe charged particle reaction data compilation. Physics Data, Karlsruhe

20. Koning AJ, Rochman D, Kopecký J, Sublet J-Ch, Bauge E, Hilaire S, Romain P, Morillon B, Duarte H, van der Marck S, Pomp S, Sjostrand H, Forrest RA, Henriksson H, Cabellos O, Goriely S, Leppanen J, Leeb H, Plompen A, Mills RW, TENDL2015: TALYS-based evaluated nuclear data library, tendl.web. psi.ch/tendl_2015/tendl2015.html. TENDL-2017: TALYS-based evaluated nuclear data library tendl.web.psi.ch/tendl_2017/tendl 2017.html

21. Koning AJ, Rochman D (2012) Modern nuclear data evaluation with the TALYS code system. Nucl Data Sheets 113:2841-2934

22. Joint Committee for Guides in Metrology (JCGM), JCGM 100: Evaluation of measurement data. Guide to the expression of uncertainty in measurement (ISO/IEC Guide 98-3). HTML version of JCGM 100 on which ISO/IEC Guide 98-3:2008 is based can be found at www.iso.org/sites/JCGM/JCGM-introducti on.htm

23. Schumaker LL (1981) Spline function: basic theory. John Wiley, New York, pp 108-118

24. Padé HE (1892) Sur la représentation approchée d' une fonction par des fractions rationnelles. Supplement to Ann Sci l'Ecole Norm Suppl Series 3 9: 3-93

25. Graves-Morris PR (ed) (1973) Padé approximants and their applications. Academic Press, New York

26. Baker GA Jr (1975) Essentials of Padé approximants. Academic Press, New York

27. Vinogradov VN, Gai EV, Rabotnov NS (1987) Analytical approximation of data in nuclear and neutron physics. Energoatomizdat, Moscow (in Russian)

28. Badikov SA, Gai EV (2003) Some sources of the underestimation of evaluated cross-section uncertainties, IAEA report INDC(NDS)-438 117-129, IAEA, Vienna, Austria. Available online at: www-nds.iaea.org/publications/indc/indc-nds-0438.pdf

29. Gai EV (2007) Some algorithms for the nuclear data evaluation and construction of the uncertainty covariance matrices. Prob Atom Sci Technol Ser Nucl Constants 1-2:56-65

30. Hermanne A, Ignatyuk AV, Capote R, Carlson BV, Engle JW, Kellett MA, Kibedi T, Kim G, Kondev FG, Hussain M, Lebeda O, Luca A, Nagai Y, Naik H, Nichols AL, Nortier FM, Suryanarayana SV, Takács S, Tárkányi FT, Verpelli M (2018) Reference cross sections for charged-particle monitor reactions. Nucl Data Sheets 148:338-382

31. Bonardi M (1987) The contribution to nuclear data for biomedical radioisotope production from the Milan Cyclotron Laboratory, Consultants' Meeting on Data Requirements for Medical Radioisotope Production, 20-24 April 1987, Tokyo, Japan (Okamoto K, ed), IAEA report INDC(NDS) 195:98-112, IAEA, Vienna, Austria (1988). Available online at: https://www-nds. iaea.org/publications/indc/indc-nds-0195.pdf

32. Otuka N, Takács S (2015) Definitions of radioisotope thick target yields. Radiochim Acta 103:1-6

33. de Waal TJ, Peisach M, Pretorius R (1971) Activation cross sections for proton-induced reactions on calcium isotopes up to 5.6 MeV. J Inorg Nucl Chem 33:2783-2789. EXFOR: O0748

34. Cheng CW, King JD (1979) Cross sections for the ${ }^{42} \mathrm{Ca}(\mathrm{p}, \gamma)^{43} \mathrm{Sc}$, ${ }^{42} \mathrm{Ca}(\alpha, \mathrm{n}){ }^{45} \mathrm{Ti}$ and ${ }^{44} \mathrm{Ca}(\mathrm{p}, \mathrm{n}){ }^{44} \mathrm{Sc}$ reactions. J Phys G: Nucl Phys 5:1261-1266

35. Mitchell LW, Anderson MR, Kennett SR, Sargood DG (1982) Cross sections and thermonuclear reaction rates for ${ }^{42} \mathrm{Ca}(\mathrm{p}$, 
$\gamma)^{43} \mathrm{Sc},{ }^{44} \mathrm{Ca}(\mathrm{p}, \gamma){ }^{45} \mathrm{Sc},{ }^{44} \mathrm{Ca}(\mathrm{p}, \mathrm{n}){ }^{44} \mathrm{Sc}$ and ${ }^{45} \mathrm{Sc}(\mathrm{p}, \mathrm{n}){ }^{45} \mathrm{Ti}$. Nucl Phys A 380:318-334. EXFOR: A0307

36. Levkovskij VN (1991) Activation cross sections for the nuclides of medium mass region $(A=40-100)$ with protons and $\alpha$-particles at medium $(E=10-50 \mathrm{MeV})$ energies. Experiment and systematics), INTER-VESTI, Moscow. EXFOR: A0510

37. Krajewski S, Cydzik I, Abbas K, Bulgheroni A, Simonelli F, Holzwarth U, Bilewicz A (2013) Cyclotron production of ${ }^{44} \mathrm{Sc}$ for clinical application. Radiochim Acta 101:333-338. EXFOR: O2136

38. Braccini S (2016) Unpublished information on studies at University Bern (private communication)

39. Duchemin C, Guertin A, Haddad F, Michel N, Métivier V (2015) Production of scandium- $44 \mathrm{~m}$ and scandium- $44 \mathrm{~g}$ with deuterons on calcium-44: cross section measurements and production yield calculations. Phys Med Biol 60:6847-6864

40. de Waal TJ, Peisach M, Pretorius R (1971) Activation cross sections for deuteron-induced reactions on calcium isotopes up to 5.5 MeV. Radiochim. Acta 15:123-127. EXFOR: R0045

41. McGee T, Rao CL, Saha GB, Yaffe L (1970) Nuclear interactions of ${ }^{45} \mathrm{Sc}$ and ${ }^{68} \mathrm{Zn}$ with protons of medium energy. Nucl Phys A 150:11-29. EXFOR: B0053

42. Ejnisman R, Goldman ID, Pascholati PR, Da Cruz MT, Olivera RM, Norman EB, Zlimen I, Wietfeldt FE, Larimer RM, Chan YD, Lesko KT, Garcia A (1996) Cross sections for ${ }^{45} \mathrm{Sc}(\mathrm{p}, 2 \mathrm{n})^{44} \mathrm{Ti}$ and related reactions. Phys Rev C 54:2047-2050. EXFOR: C0438

43. Daraban L, Adam-Rebeles R, Hermanne A, Tárkányi F, Takács $\mathrm{S}$ (2009) Cross sections for ${ }^{45} \mathrm{Sc}(\mathrm{p}, 2 \mathrm{n})^{44} \mathrm{Ti}$ and related reactions: Study of the excitation functions for ${ }^{43} \mathrm{~K},{ }^{43,44,44 \mathrm{~m}} \mathrm{Sc}$ and ${ }^{44} \mathrm{Ti}$ by proton irradiation on ${ }^{45} \mathrm{Sc}$ up to $37 \mathrm{MeV}$. Nucl Instrum Methods Phys Res B 267:755-759. EXFOR: D4211

44. Hermanne A, Adam-Rebeles R, Tárkányi F, Takács S, Takács MP, Csikái J, Ignatyuk A (2012) Cross sections of deuteron induced reactions on ${ }^{45} \mathrm{Sc}$ up to $50 \mathrm{MeV}$ : experiments and comparison with theoretical codes. Nucl Instrum Methods Phys Res B 270:106-115. EXFOR: D4257

45. Tanaka S, Furukawa M, Chiba M (1972) Nuclear reactions of nickel with protons up to $56 \mathrm{MeV}$. J Inorg Nucl Chem 34:24192426. EXFOR: B0020

46. Steyn GF, Mills SJ, Nortier FM, Simpson BRS, Meyer BR (1990) Production of ${ }^{52} \mathrm{Fe}$ via proton-induced reactions on manganese and nickel. Appl Radiat Isot 41:315-325. EXFOR: A0497

47. Titarenko Yu E, Batyaev VF, Titarenko AYu, Butko MA, Pavlov KV, Florya SN, Tikhonov RS, Zhivun VM, Ignatyuk AV, Mashnik SG, Leray S, Boudard A, Cugnon J, Mancusi D, Yariv Y, Nishihara K, Matsuda N, Kumawat H, Mank G, Gudowski W (2011) Measurement and simulation of the cross sections for nuclide production in Nb-93 and Ni-nat targets irradiated with 0.04- to 2.6-GeV protons. Yadernaya Fizika 74:548-560. EXFOR: A0906

48. Hermanne A, Adam-Rebeles R, Tárkányi F, Takács S, Ditrói F (2015) Proton and deuteron induced reactions on ${ }^{\text {nat }} \mathrm{Ga}$ : Experimental and calculated excitation functions. Nucl Instrum Methods Phys Res B 359:145-154. EXFOR: D4343

49. Suzuki K (1985) Production of ${ }^{52} \mathrm{Fe}$ by the ${ }^{55} \mathrm{Mn}(\mathrm{p}, 4 \mathrm{n}){ }^{52} \mathrm{Fe}$ reaction and milking of ${ }^{52 \mathrm{~m}} \mathrm{Mn}$ from ${ }^{52} \mathrm{Fe}$. Radioisotopes 34:537-542. EXFOR: E2397

50. Deptula C, Kim SH, Knotek O, Mikolajewski S, Popinenkova LM, Rurarz E, Zaitseva NG (1990) Excitation functions and yields of some nuclear reactions induced by $100 \mathrm{MeV}$ energy protons in $\mathrm{Mn}$ and Co targets-production of Fe-52. Nukleonika 35:49-61. EXFOR: A0573

51. Zaitseva NG, Deptula C, Knotek O, Kim SK, Mikolaevsky S, Mikecz P, Rurarz E, Khalkin VA, Konov VA, Popinenkova LM (1991) Cross sections for the $100 \mathrm{MeV}$ proton-induced nuclear reactions and yields of some radionuclides used in nuclear medicine. Radiochim Acta 54:57-72. EXFOR: A0918

52. Akiha F, Aburai T, Nozaki T, Murakami Y (1972) Yield of ${ }^{52} \mathrm{Fe}$ for the reactions of ${ }^{3} \mathrm{He}$ and on chromium. Radiochim Acta 18:108-111

53. Chowdhury DP, Pal S, Saha SK, Gangadharan S (1995) Determination of cross section of $\alpha$-induced nuclear reaction on natural $\mathrm{Cr}$ and $\mathrm{Zr}$ by stacked foil activation for thin layer activation analysis. Nucl Instrum Methods Phys Res B103:261-266. EXFOR: A0204

54. Hermanne A, Adam-Rebeles R, Tárkányi F, Takács S (2015) Alpha particle induced reactions on ${ }^{\text {nat }} \mathrm{Cr}$ up to $39 \mathrm{MeV}$. Nucl Instrum Methods Phys Res B356-357:28-41. EXFOR: D4335

55. Boehm F, Marmier P, Preiswerk P (1952) Relative cross sections for the excitation of isomers and ground states by $(\mathrm{p}, \mathrm{n})$ reaction. Helv Phys Acta 25:599-604. EXFOR: O2127

56. Blosser HG, Handley TH (1955) Survey of (p, n) reactions at 12 MeV. Phys Rev 100:1340-1344. EXFOR: B0052

57. Taketani H, Alford WP (1962) (p,n) Cross Sections on $\mathrm{Ti}^{47}, \mathrm{~V}^{51}$, $\mathrm{Cr}^{52}, \mathrm{Co}^{59}$, and $\mathrm{Cu}^{63}$ from 4 to $6.5 \mathrm{MeV}$. Phys Rev 125:291-294. EXFOR: B0051

58. Wing J, Huizenga JR (1962) ( $\mathrm{p}, \mathrm{n})$ Cross sections of $\mathrm{V}^{51}, \mathrm{Cr}^{52}$, $\mathrm{Cu}^{63}, \mathrm{Cu}^{65}, \mathrm{Ag}^{107}, \mathrm{Ag}^{109}, \mathrm{Cd}^{111}, \mathrm{Cd}^{114}$, and $\mathrm{La}^{139}$ from 5 to 10.5 MeV. Phys Rev 128:280-290. EXFOR: T0124

59. Barrandon JN, Debrun JL, Kohn A, Spear RH (1975) Étude du dosage de $\mathrm{Ti}, \mathrm{V}, \mathrm{Cr}, \mathrm{Fe}, \mathrm{Ni}, \mathrm{Cu}$ et $\mathrm{Zn}$ par activation avec des protons d'énergie limitée a $20 \mathrm{MeV}$. Nucl Instrum Methods 127:269-278. EXFOR: O0086

60. Muminov VA, Mukhammedov S, Vasidov A (1980) Possibilities of proton-activation analysis for determining the content of elements from short-lived radionuclides. Atomnaya Energiya 49:101-105. EXFOR: A0085

61. Skakun EA, Baty VG, Rakivnenko Yu N, Rastrepin OA (1986) Investigation of cross sections of reactions ${ }^{52} \mathrm{Cr}(\mathrm{p}, \mathrm{n})^{52 \mathrm{~g} 52 \mathrm{~m}} \mathrm{Mn}$ and ${ }^{54} \mathrm{Cr}(\mathrm{p}, \mathrm{n}){ }^{54} \mathrm{Mn}$ at incident energies of $5-9 \mathrm{MeV}$. Izv Rossiiskoi Akademii Nauk Ser Fiz 50:2043. EXFOR: A0292

62. West HI, Lanier RG Jr, Mustafa MG (1987) ${ }^{52} \mathrm{Cr}(\mathrm{p}$, $\mathrm{n}{ }^{52 \mathrm{~g}} \mathrm{Mn},{ }^{52 \mathrm{~m}} \mathrm{Mn}$ and ${ }^{52} \mathrm{Cr}(\mathrm{d}, 2 \mathrm{n}){ }^{52 \mathrm{~g}} \mathrm{Mn},{ }^{52 \mathrm{~m}} \mathrm{Mn}$ excitation functions. Phys Rev C 35:2067-2076. EXFOR: C0336

63. Cogneau M, Gilly L, Cara J (1966) Absolute cross sections and excitation functions for deuteron induced reactions on chromium between 2 and $12 \mathrm{MeV}$. Nucl Phys 79:203-208. EXFOR: O2033

64. Tanaka S, Furukawa M (1959) Excitation functions for (p, n) reactions with titanium, vanadium, chromium, iron and nickel up to $E_{p}=14$ MeV. J Phys Soc Jpn 14:1269-1275. EXFOR: B0043

65. Lindner B, James RA (1959) Cross sections for nuclear reactions involving nuclear isomers. Phys Rev 114:322-325. EXFOR: C0698

66. Antropov AE, Gusev VP, Zarubin PP, Ioannu PD, Padalko VYu (1980) Measurement of the total cross section for the $(p, n)$ reaction on medium mass atomic nuclei with $6 \mathrm{MeV}$ protons. 30th Conference nuclear spectroscopy and nuclear structure, USSR, Leningrad, p 316. EXFOR: A0072

67. Buchholz M, Spahn I, Scholten B, Coenen HH (2013) Crosssection measurements for the formation of manganese- 52 and its isolation with a non-hazardous eluent. Radiochim Acta 101:491500. EXFOR: O2176

68. Wooten AL, Lewis BC, Lapi SE (2015) Cross-sections for (p, $x$ ) reactions on natural chromium for the production of ${ }^{52,52 \mathrm{~m}, 54} \mathrm{Mn}$ radioisotopes. Appl Radiat Isot 96:154-161. EXFOR: C2145

69. Zherebchevsky VI, Alekseev IE, Gridnev KA, Krymov EB, Lazareva TV, Maltsev NA, Panin RB, Prokofyev NA, Torilov SY, Shtamburg AI (2016) The study of the nuclear reactions for the production of antimony isotopes. Izv Rossiiskoi Akademii Nauk Ser Fiz 80:975. EXFOR: F1298 
70. Burgus WH, Cowan GA, Hadley JW, Hess W, Shull T, Stevenson ML, York HF (1954) Cross sections for the reactions $\mathrm{Ti}^{48}(\mathrm{~d}, 2 \mathrm{n})$ $\mathrm{V}^{48} ; \mathrm{Cr}^{52}(\mathrm{~d}, 2 \mathrm{n}) \mathrm{Mn}^{52}$; and $\mathrm{Fe}^{56}(\mathrm{~d}, 2 \mathrm{n}) \mathrm{Co}^{56}$. Phys Rev 95:750-751. EXFOR: D4102

71. Xiaowu C, Zhenxia W, Zhenjie W, Jinqing Y (1966) Some measurements of deuteron induced excitation function at $13 \mathrm{MeV}$. Acta Physica Sinica 22:250-252. EXFOR: S0060

72. Nassiff SJ, Münzel H (1973) Cross sections for the reactions ${ }^{66} \mathrm{Zn}(\mathrm{d}, \mathrm{n}){ }^{67} \mathrm{Ga},{ }^{52} \mathrm{Cr}(\mathrm{d}, 2 \mathrm{n}){ }^{52 \mathrm{~g}} \mathrm{Mn}$ and ${ }^{186} \mathrm{~W}(\mathrm{~d}, 2 \mathrm{n}){ }^{186} \mathrm{Re}$. Radiochim Acta 19:97-99. EXFOR: A0202

73. Hermanne A, Adam RR, Tárkányi F, Takács S, Takács M, Ignatyuk A (2011) Cross sections of deuteron induced reactions on ${ }^{\text {nat }} \mathrm{Cr}$ up to $50 \mathrm{MeV}$ : Experiments and comparison with theoretical codes. Nucl Instrum Methods Phys Res B 269:2563-2571. EXFOR: D4252

74. Kaufman S (1960) Reactions of protons with Ni-58 and Ni-60. Phys Rev 117:1532-1538. EXFOR: B0055

75. Ewart HA, Blann M (1964) Private communication. EXFOR: C1012. Ewart HA, Blann M (1964) Data taken from McGowen et al., ORNL report ORNL-CPX-2. EXFOR: C1012

76. Haasbroek FJ, Steyn J, Neirinckx RD, Burdzik GF, Cogneau M, Wanet P (1977) Excitation functions and thick target yields for radioisotopes induced in natural $\mathrm{Mg} \mathrm{Co}, \mathrm{Ni}$ and $\mathrm{Ta} \mathrm{By}$ medium energy protons. Council of Scientific and Industrial Research, report no. 89, 1976, Pretoria, South Africa. Int J Appl Radiat Isot 28:533-534. EXFOR: B0098

77. Brinkman GA, Helmer J, Lindner L (1977) Nickel and copper foils as monitors for cyclotron beam intensities. Radiochem Radioanal Lett 28:9-19. EXFOR: D0162

78. Michel R, Weigel H, Herr W (1978) Proton-induced reactions on nickel with energies between 12 and $45 \mathrm{MeV}$. Z Phys A 286:393400. EXFOR: B0083

79. Michel R, Brinkmann G (1980) On the depth-dependent production of radionuclides (A between 44 and 59) by solar protons in extraterrestrial matter. J Radioanal Chem 59:467-510. EXFOR: A0145

80. Tárkányi F, Szelecsényi F, Kopecký P (1991) Excitation functions of proton induced nuclear reactions on natural nickel for monitoring beam energy and intensity. Appl Radiat Isot 42:513517. EXFOR: D4002

81. Sonck M, Van Hoyweghen J, Hermanne A (1996) Determination of the external beam energy of a variable energy multiparticle cyclotron. Appl Radiat Isot 47:445-449. EXFOR: D0393

82. Michel R, Bodemann R, Busemann H, Daunke R, Gloris M, Lange H-J, Klug B, Krins A, Leya I, Lüpke M, Neumann S, Reinhardt H, Schnatz-Buttgen M, Herpers U, Schiekel T, Sudbrock F, Holmqvist B, Conde H, Malmborg P, Suter M, Dittrich-Hannen B, Kubik P-W, Synal H-A, Filges D (1997) Cross sections for the production of residual nuclides by low- and medium-energy protons from the target elements $\mathrm{C}, \mathrm{N}, \mathrm{O}, \mathrm{Mg}, \mathrm{Al}, \mathrm{Si}, \mathrm{Ca}, \mathrm{Ti}$, $\mathrm{V}, \mathrm{Mn}, \mathrm{Fe} \mathrm{Co}, \mathrm{Ni}, \mathrm{Cu}, \mathrm{Sr}, \mathrm{Y}, \mathrm{Zr}, \mathrm{Nb}, \mathrm{Ba}$ and Au. Nucl Instrum Methods Phys Res B 129:153-193. EXFOR: O0276

83. Reimer P, Qaim SM (1998) Excitation functions of proton induced reactions on highly enriched ${ }^{58} \mathrm{Ni}$ with special relevance to the production of ${ }^{55} \mathrm{Co}$ and ${ }^{57} \mathrm{Co}$. Radiochim Acta 80:113-120. EXFOR: D4078

84. Al-Saleh FS, Al Mugren KS, Azzam A (2007) Excitation functions of $(\mathrm{p}, \mathrm{x})$ reactions on natural nickel between proton energies of 2.7 and $27.5 \mathrm{MeV}$. Appl Radiat Isot 65:104-113. EXFOR: $\mathrm{O} 1503$

85. Khandaker MU, Kim K, Lee M, Kim KS, Kim G (2011) Excitation functions of $(\mathrm{p}, \mathrm{x})$ reactions on natural nickel up to $40 \mathrm{MeV}$. Nucl Instrum Methods Phys Res B 269:1140-1149. EXFOR: D7002

86. Jost CU, Griswold JR, Bruffey SH, Mirzadeh S, Stracener DW, Williams CL (2013) Measurement of cross sections for the
${ }^{232} \mathrm{Th}(\mathrm{p}, 4 \mathrm{n}){ }^{229} \mathrm{~Pa}$ reaction at low proton energies. Conf Proc Am Inst Phys 1525:520-524. EXFOR: C2048

87. Amjed N, Tárkányi F, Hermanne A, Ditrói F, Takács S, Hussain M (2014) Activation cross-sections of proton induced reactions on natural Ni up to $65 \mathrm{MeV}$. Appl Radiat Isot 92:73-84. EXFOR: D4301

88. Vlasov NA, Kalinin SP, Ogloblin AA, Pankramov VM, Rudakov VP, Serikov IN, Sidorov VA (1957) Excitation functions for the reactions Mg-24(d, $\alpha) \mathrm{Na}-22, \mathrm{Fe}-54(\mathrm{~d}, \alpha) \mathrm{Mn}-52, \mathrm{Fe}-54(\mathrm{~d}, \mathrm{n})$ Co-55, and Zn-66(d,2n)Ga-66. Atomnaya Energiya 2:189-192. EXFOR: F1220

89. Clark JW, Fulmer CB, Williams IR (1969) Excitation function for radioactive nuclides produced by deuteron-induced reactions in iron. Phys Rev 179:1104-1108. EXFOR: T0199

90. Coetzee PP, Peisach M (1972) Activation cross sections for deuteron-induced reactions on some elements of the first transition series, up to $5.5 \mathrm{MeV}$. Radiochim Acta 17:1-6. EXFOR: D4054

91. Zhenlan T, Fuying Z, Huiyuan Q, Gongoing W (1984) Excitation function of deuteron induced reactions on natural iron. Atom Energy Sci Technol 18:506. EXFOR: S0015

92. Zhao Wenrong L, Weixiang Hanlin, Yu, Jiantao C (1995) Excitation functions for reactions induced by deuteron in iron. Chin $\mathrm{J}$ Nucl Phys (Beijing) 17:163. EXFOR: S0044

93. Zaman MR, Qaim SM (1996) Excitation functions of (d, n) and $(\mathrm{d}, \alpha)$ reactions on ${ }^{54} \mathrm{Fe}$ : relevance to the production of high purity ${ }^{55} \mathrm{Co}$ at a small cyclotron. Radiochim Acta 75:59-63. EXFOR: D4131

94. Hermanne A, Sonck M, Takács S, Tárkányi F (2000) Experimental study of excitation functions for some reactions induced by deuterons $(10-50 \mathrm{MeV})$ on natural Fe and Ti. Nucl Instrum Methods Phys Res B161-163:178-185. EXFOR: D4082

95. Nakao M, Hori J, Ochiai K, Kubota N, Sato S, Yamauchi M, Ishioka NS, Nishitani T (2006) Measurements of deuteron-induced activation cross-sections for IFMIF accelerator structural materials. Nucl Instrum Methods Phys Res A 562:785-788. EXFOR: E1988

96. Király B, Takács S, Ditrói F, Tárkányi F, Hermanne A (2009) Evaluated activation cross sections of longer-lived radionuclides produced by deuteron induced reactions on natural iron up to $10 \mathrm{MeV}$. Nucl Instrum Methods Phys Res B 267:15-22. EXFOR: D4185

97. Zavorka L, Šimečková E, Honusek M, Katovsky K (2011) The activation of Fe by deuterons of energies up to $20 \mathrm{MeV}$. J Korean Phys Soc 59:1961-1964

98. Cohen BL, Newman E (1955) ( $p$, pn) And $(p, 2 n)$ cross sections in medium weight elements. Phys Rev 99:718-723. EXFOR: B0050

99. Williams IR, Fulmer CB (1967) Excitation functions for radioactive isotopes produced by protons below $60 \mathrm{MeV}$ on $\mathrm{Al}, \mathrm{Fe}$, and $\mathrm{Cu}$. Phys Rev 162:1055-1061. EXFOR: B0073

100. Jenkins IL, Wain AG (1970) Excitation functions for the bombardment of ${ }^{56} \mathrm{Fe}$ with protons. J Inorg Nucl Chem 32:14191425. EXFOR: B0041

101. Brodzinski RL, Rancitelli LA, Cooper JA, Wogman NA (1971) High-energy proton spallation of iron. Phys Rev C 4:1257-1265. EXFOR: C0272

102. Michel R, Brinkmann G, Weigel H, Herr W (1979) Measurement and hybrid-model analysis of proton-induced reactions with $\mathrm{V}$, Fe and Co. Nucl Phys A 322:40-60. EXFOR: A0146

103. Lagunas-Solar MC, Jungerman JA (1979) Cyclotron production of carrier-free Cobalt-55, a new positron-emitting label for bleomycin. Int J Appl Radiat Isot 30:25-32. EXFOR: A0182

104. Zhuravlev BV, Grusha OV, Ivanova SP, Trykova VI, Shubin Y (1984) Analysis of neutron spectra in 22-MeV proton interactions with nuclei. Yad Fiz 39:264-271. EXFOR: A0271 
105. Zhao Wenrong L, Weixiang Hanlin, Yu (1993) Measurement of cross sections by bombarding Fe with protons up to $19 \mathrm{MeV}$. Chin J Nucl Phys (Beijing) 15:337-340. EXFOR: A0600

106. Daum E (1997) Investigation of light ion induced activation cross sections in iron. 1.1 Proton induced activation cross sections. Germany/IAEA report INDC(GER)-043 (1997) 4-5, IAEA, Vienna, Austria. Available online at: https://www-nds.iaea.org/ publications/indc/indc-ger-0043.pdf. PhD thesis, Forschungszentrum Karlsruhe report no. 5833. EXFOR: O0498

107. Ditrói F, Tárkányi F, Csikái J, Uddin MS, Hagiwara M, Baba M (2004) Investigation of activation cross sections of the proton induced nuclear reactions on natural iron at medium energies. In: International conference on nuclear data for science and technology, vol 769. Santa Fe, NM, USA, p 1011. EXFOR: D4181

108. Al-Abyad M, Comsan MNH, Qaim SM (2009) Excitation functions of proton-induced reactions on ${ }^{\text {nat }} \mathrm{Fe}$ and enriched ${ }^{57} \mathrm{Fe}$ with particular reference to the production of ${ }^{57} \mathrm{Co}$. Appl Radiat Isot 67:122-128. EXFOR: D0500

109. Kim K, Khandaker MU, Naik H, Kim G (2014) Excitation functions of proton induced reactions on ${ }^{\text {nat }} \mathrm{Fe}$ in the energy region up to $45 \mathrm{MeV}$. Nucl Instrum Methods Phys Res B 322:63-69. EXFOR: D7007

110. Blaser JP, Boehm F, Marmier P, Scherrer P (1951) Anregungsfunktionen und Wirkungsquerschnitte der ( $\mathrm{p}, \mathrm{n})$-Reaktion (II). Helv Phys Acta 24:441-464. EXFOR: P0033

111. Johnson CH, Trail CC, Galonsky A (1964) Thresholds for $(\mathrm{p}, \mathrm{n})$ reactions on 26 intermediate-weight nuclei. Phys Rev 136:B1719-B1729. EXFOR: T0126

112. Tingwell CIW, Hansper VY, Tims SG, Scott AF, Sargood DG (1988) Cross sections of proton induced reactions on ${ }^{61} \mathrm{Ni}$. Nucl Phys A 480:162-174. EXFOR: F0833

113. Szelecsényi F, Blessing G, Qaim SM (1993) Excitation functions of proton induced nuclear reactions on enriched ${ }^{61} \mathrm{Ni}$ and ${ }^{64} \mathrm{Ni}$ : Possibility of production of no-carrier-added ${ }^{61} \mathrm{Cu}$ and ${ }^{64} \mathrm{Cu}$ at a small cyclotron. Appl Radiat Isot 44:575-580. EXFOR: D4020

114. Singh BP, Sharma MK, Musthafa MM, Bhardwaj HD, Prasad $\mathrm{R}$ (2006) A study of pre-equilibrium emission in some protonand alpha-induced reactions. Nucl Instrum Methods Phys Res A 562:717-720. EXFOR: D6012

115. Hermanne A, Adam-Rebeles R, Tárkányi F, Takács S (2015) Excitation functions of proton induced reactions on ${ }^{\text {nat }} O$ s up to $65 \mathrm{MeV}$ : experiments and comparison with results from theoretical codes. Nucl Instrum Methods Phys Res B 45:58-68. EXFOR: D4324

116. Uddin MS, Chakraborty AK, Spellerberg S, Shariff MA, Das S, Rashid MA, Spahn I, Qaim SM (2016) Experimental determination of proton induced reaction cross sections on ${ }^{\text {nat }} \mathrm{Ni}$ near threshold energy. Radiochim Acta 104:305-314. EXFOR: D0802

117. Uddin MS, Sudar S, Spahn I, Shariff MA, Qaim SM (2016) Excitation function of the ${ }^{60} \mathrm{Ni}(\mathrm{p}, \gamma){ }^{61} \mathrm{Cu}$ reaction from threshold to $16 \mathrm{MeV}$. Phys Rev C 93:044606. EXFOR: D0808

118. Cogneau M, Gilly LJ, Cara J (1967) Absolute cross sections and excitation functions for deuteron-induced reactions on the nickel isotopes between 2 and $12 \mathrm{MeV}$. Nucl Phys A 99:686-694. EXFOR: D0076

119. Takács S, Sonck M, Azzam A, Hermanne A, Tárkányi F (1997) Activation cross section measurements of deuteron induced reactions on ${ }^{\text {nat }} \mathrm{Ni}$ with special reference to beam monitoring and production of ${ }^{61} \mathrm{Cu}$ for medical purpose. Radiochim Acta 76:15-24. EXFOR: D4045

120. Hermanne A, Tárkányi F, Takács S, Kovalev SF, Ignatyuk A (2007) Activation cross sections of the ${ }^{64} \mathrm{Ni}(\mathrm{d}, 2 \mathrm{n})$ reaction for the production of the medical radionuclide ${ }^{64} \mathrm{Cu}$. Nucl Instrum Methods Phys Res B 258:308-312. EXFOR: D4182

121. Hermanne A, Takács S, Adam-Rebeles R, Tárkányi F, Takács MP (2013) New measurements and evaluation of database for deuteron induced reaction on $\mathrm{Ni}$ up to $50 \mathrm{MeV}$. Nucl Instrum Methods Phys Res B 299:8-23. EXFOR: D4282

122. Cohen BL, Newman E, Charpie RA, Handley TH (1954) (p, pn) and (p, $\alpha$ ) excitation functions. Phys Rev 94:620-624. EXFOR: B0072

123. Szelecsényi F, Kovács Z, Suzuki K, Okada K, van der Walt TN, Steyn GF, Mukherjee S (2005) Production possibility of ${ }^{61} \mathrm{Cu}$ using proton induced nuclear reactions on zinc for PET studies. J Radioanal Nucl Chem 263:539-546. EXFOR: E1975

124. Uddin MS, Khandaker MU, Kim KS, Lee YS, Kim GN (2007) Excitation functions of the proton induced nuclear reactions on

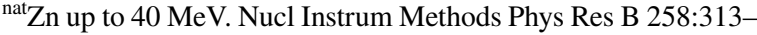
320. EXFOR: O1600

125. Asad AH, Chan S, Morandeau L, Cryer D, Smith SV, Price RI (2014) Excitation functions of ${ }^{\text {nat }} \mathrm{Zn}(p, x)$ nuclear reactions with proton beam energy below $18 \mathrm{MeV}$. Appl Radiat Isot 94:67-71. EXFOR: D0734

126. Gyurky G, Fulop Z, Halasz Z, Kiss GG, Szǔcs T (2014) Direct study of the $\alpha$-nucleus optical potential at astrophysical energies using the ${ }^{64} \mathrm{Zn}(p, \alpha){ }^{61} \mathrm{Cu}$ reaction. Phys Rev C 90:052801. EXFOR: D4319

127. Ghoshal SN (1950) An experimental verification of the theory of compound nucleus. Phys Rev 80:939-942. EXFOR: B0017

128. Greene MW, Lebowitz E (1972) Proton reactions with copper for auxiliary cyclotron beam monitoring. Int J Appl Radiat Isot 23:342-344. EXFOR: B0074

129. Grütter A (1982) Excitation functions for radioactive isotopes produced by proton bombardment of $\mathrm{Cu}$ and $\mathrm{Al}$ in the energy range of 16 to $70 \mathrm{MeV}$. Nucl Phys A 383:98-108. EXFOR: D0505

130. Greenwood LR, Smither RK (1984) Measurement of Cu spallation cross sections at IPNS, US Dept. of Energy, Fusion Energy Series, vol 18, no. 0046, p 11. EXFOR: C0537

131. Kopecký J (1985) Proton beam monitoring via the $\mathrm{Cu}(\mathrm{p}$, $\mathrm{x}){ }^{58} \mathrm{Co},{ }^{63} \mathrm{Cu}(\mathrm{p}, 2 \mathrm{n}){ }^{62} \mathrm{Zn}$ and ${ }^{65} \mathrm{Cu}(\mathrm{p}, \mathrm{n}){ }^{65} \mathrm{Zn}$ reactions in copper. Int J Appl Radiat Isot 36:657-661. EXFOR: A0333

132. Aleksandrov VN, Semenova MP, Semenov VG (1987) Production cross section of radionuclides in $(\mathrm{p}, \mathrm{x})$ reactions at copper and nickel nuclei. Atomnaya Energiya 62:411-413. EXFOR: A0351

133. Mills SJ, Steyn GF, Nortier FM (1992) Experimental and theoretical excitation functions of radionuclides produced in proton bombardment of copper up to $200 \mathrm{MeV}$. Appl Radiat Isot 43:1019-1030. EXFOR: A0507

134. Hermanne A, Szelecsényi F, Sonck M, Takács S, Tárkányi F, van den Winkel P (1999) New cross section data on ${ }^{68} \mathrm{Zn}(\mathrm{p}, 2 \mathrm{n}){ }^{67} \mathrm{Ga}$ and ${ }^{n a t} \mathrm{Zn}(\mathrm{p}, \mathrm{xn}){ }^{67} \mathrm{Ga}$ nuclear reactions for the development of a reference data base. J Radioanal Nucl Chem 240:623-630. EXFOR: D4088

135. Szelecsényi F, Tárkányi F, Takács $\mathrm{S}$, Hermanne A, Sonck M, Shubin Yu, Mustafa MG, Zhuang Y (2001) Excitation function for the ${ }^{\text {nat }} \mathrm{Ti}(\mathrm{p}, \mathrm{x})^{48} \mathrm{~V}$ nuclear process: evaluation and new measurements for practical applications. Nucl Instrum Methods Phys Res B 174:47-64. EXFOR: D4083

136. Takács S, Tárkányi F, Sonck M, Hermanne A (2002) New crosssections and intercomparison of proton monitor reactions on $\mathrm{Ti}$, $\mathrm{Ni}$ and $\mathrm{Cu}$. Nucl Instrum Methods Phys Res B 188:106-111. EXFOR: D4106

137. Uddin MS, Hagiwara M, Tárkányi F, Ditrói F, Baba M (2004) Experimental studies on the proton-induced activation reactions of molybdenum in the energy range $22-67 \mathrm{MeV}$. Appl Radiat Isot 60:911-920. EXFOR: E1894

138. Buthelezi EZ, Nortier FM, Schroeder IW (2006) Excitation functions for the production of ${ }^{82} \mathrm{Sr}$ by proton bombardment of ${ }^{n a t} \mathrm{Rb}$ at energies up to $100 \mathrm{MeV}$. Appl Radiat Isot 64:915-924. EXFOR: O1424 
139. Al-Saleh FS, Al-Harbi AA, Azzam A (2006) Excitation functions of proton induced nuclear reactions on natural copper using a medium-sized cyclotron. Radiochim Acta 94:391-396. EXFOR: D0422

140. Khandaker MU, Uddin MS, Kim KS, Lee YS, Kim GN (2007) Measurement of cross-sections for the (p, xn) reactions in natural molybdenum. Nucl Instrum Methods Phys Res B 262:171-181. EXFOR: D04461

141. Siiskonen T, Huikari J, Haavisto T, Bergman J, Heselius S-J, Lill J-O, Lönnroth T, Peräjärvi K (2009) Excitation functions of proton-induced reactions in ${ }^{\text {nat }} \mathrm{Cu}$ in the energy range 7-17 MeV. Appl Radiat Isot 67:2037-2039. EXFOR: D0549

142. Tárkányi F, Ditrói F, Hermanne A, Takács S, Ignatyuk AV (2012) Investigation of activation cross-sections of proton induced nuclear reactions on ${ }^{\text {nat }} \mathrm{Mo}$ up to $40 \mathrm{MeV}$ : new data and evaluation. Nucl Instrum Methods Phys Res B 280:45-73. EXFOR: D4264

143. Hermanne A, Tárkányi F, Takács S (2013) Experiments onTi, Cu and $\mathrm{Ni}$ foils with protons of 36,25 and $17 \mathrm{MeV}$ at VUB cyclotron (private communication)

144. Lebeda $O$ (2014) Cross-section values for proton and deuteron beam monitoring (private communication at Second Research Coordination Meeting, IAEA, Vienna, Austria)

145. Shahid M, Kim K, Naik H, Zaman M, Yang SC, Kim GN (2015) Measurement of excitation functions in proton induced reactions on natural copper from their threshold to $43 \mathrm{MeV}$. Nucl Instrum Methods Phys Res B 342:305-313. EXFOR: D7015

146. Lebeda $\mathrm{O}$ (2016) New cross-sections for the ${ }^{\text {nat }} \mathrm{Ti}(p, x)^{48} \mathrm{~V}-{ }^{46} \mathrm{Sc}$ reactions (private communication)

147. Bartell FO, Helmholz AC, Softky SD, Stewart DB (1950) Excitation functions for spallation reactions on $\mathrm{Cu}$. Phys Rev 80:10061010. EXFOR: P0069

148. Fulmer CB, Williams IR (1970) Excitation functions for radioactive nuclides produced by deuteron-induced reactions in copper. Nucl Phys A 155:40-48. EXFOR: B0121

149. Takács S, Tárkányi F, Király B, Hermanne A, Sonck M (2006) Evaluated activation cross sections of longer-lived radionuclides produced by deuteron-induced reaction on natural copper. Nucl Instrum Methods Phys Res B 251:56-65. EXFOR: D4176

150. Šimečková E, Bém P, Honusek M, Stefanik M, Fischer U, Simakov SP, Forrest RA, Koning AJ, Sublet J-Ch, Avrigeanu M, Roman FL, Avrigeanu VA (2011) Low and medium energy deuteron-induced reactions on ${ }^{63,65} \mathrm{Cu}$ nuclei. Phys Rev C 84:014605. EXFOR: D0653

151. Adam-Rebeles R, Van den Winkel P, Hermanne A (2011) Activation cross section of deuteron induced reactions on natural thallium for the production of ${ }^{203} \mathrm{~Pb}$. J Korean Phys Soc 59:19751978. EXFOR: D4249

152. Adam-Rebeles R, Van den Winkel P, Hermanne A, Tárkányi F, Takács S (2012) Experimental excitation functions of deuteron induced reactions on natural thallium up to $50 \mathrm{MeV}$. Nucl Instrum Methods Phys Res B 288:94-101. EXFOR: D4270

153. Hermanne A, Adam-Rebeles R, Van den Winkel P, Tárkányi F, Takács S (2014) Activation of ${ }^{112} \mathrm{Cd}$ by deuteron induced reactions up to $50 \mathrm{MeV}$ : an alternative for ${ }^{111}$ In production? Nucl Instrum Methods Phys Res B 339:26-33. EXFOR: D4314

154. Tanaka S (1960) Reactions of nickel with alpha-particles. J Phys Soc Jpn 15:2159-2167. EXFOR: P0037

155. Neirinckx RD (1977) Excitation function for the ${ }^{60} \mathrm{Ni}(\alpha, 2 \mathrm{n}){ }^{62} \mathrm{Zn}$ reaction and production of ${ }^{62} \mathrm{Zn}$ bleomycin. Int J Appl Radiat Isot 28:808-809. EXFOR: B0164

156. Muramatsu H, Shirai E, Nakahara H, Murakami Y (1978) Alpha particle bombardment of natural nickel target for the production of ${ }^{61} \mathrm{Cu}$. Int J Appl Radiat Isot 29:611-614. EXFOR: B0128
157. Michel R, Brinkmann G, Stuck R (1983) Integral excitation functions of $\alpha$-induced reactions on titanium, iron and nickel. Radiochim Acta 32:173-189. EXFOR: A0148

158. Takács S, Tárkányi F, Kovács Z (1996) Excitation function of alpha-particle induced nuclear reactions on natural nickel. Nucl Instrum Methods Phys Res B 113:424-428. EXFOR: D4050

159. Singh NL, Mukherjee S, Gadkari MS (2005) Excitation functions of alpha induced reactions on natural nickel up to $50 \mathrm{MeV}$. Int. J. Mod. Phys. E 14:611-629. EXFOR: O1287

160. Yadav A, Singh PP, Sharma MK, Singh DP, Unnati SB, Prasad R, Musthafa MM (2008) Large pre-equilibrium contribution in $\alpha+{ }^{\text {nat }} \mathrm{Ni}$ interactions at $\approx 8-40 \mathrm{MeV}$. Phys Rev C78:044606. EXFOR: D6067

161. Antropov AE, Zarubin PP, Aleksandrov Yu A, Gorshkov IY (1985) Study of the cross section for the reactions (p, n), $(\alpha$, $\mathrm{pn}),(\alpha, \mathrm{xn})$ on medium weight nuclei. In: 35 th Conference nuclear spectroscopy and nuclear structure, Leningrad, p 369. EXFOR: O0076

162. Antropov AE, Gusev VP, Zhuravlev Y, Zarubin PP, Kolozhvary AA, Smirnov AV (1992) Total cross sections of (p, n) reaction on the nuclei of isotopes nickel and zinc at $E(p)=5-6 \mathrm{MeV}$. Izv Rossiiskoi Akademii Nauk Ser Fiz 56:198. EXFOR: A0543

163. Piel H, Qaim SM, Stocklin G (1992) Excitation function of (p, $\mathrm{xn}$ )-reactions on ${ }^{\text {nat }} \mathrm{Ni}$ and highly enriched ${ }^{62} \mathrm{Ni}$ : possibility of production of medically important radioisotope ${ }^{62} \mathrm{Cu}$ at a small cyclotron. Radiochim Acta 57:1-5. EXFOR: D0056

164. Blaser JP, Boehm F, Marmier P, Peaslee DC (1951) Fonctions d'excitation de la reaction (p, n). Helv Phys Acta 24:3-38. EXFOR: B0048

165. Howe HA (1958) (p, n) cross sections of copper and zinc. Phys Rev 109:2083-2085. EXFOR: B0060

166. Hille M, Hille P, Uhl M, Weisz W (1972) Excitation functions of $(\mathrm{p}, \mathrm{n})$ and $(\alpha, \mathrm{n})$ reactions on $\mathrm{Ni}, \mathrm{Cu}$ and $\mathrm{Zn}$. Nucl Phys A 198:625-640. EXFOR: B0058

167. Little FE, Lagunas-Solar MC (1983) Cyclotron production of ${ }^{67} \mathrm{Ga}$. Cross sections and thick-target yields for the ${ }^{67} \mathrm{Zn}(\mathrm{p}, \mathrm{n})$ and ${ }^{68} \mathrm{Zn}(\mathrm{p}, 2 \mathrm{n})$ reactions. Int J Appl Radiat Isot 34:631-637. EXFOR: A0321

168. Kopecký $\mathrm{P}$ (1990) Cross sections and production yields of ${ }^{66} \mathrm{Ga}$ and ${ }^{67} \mathrm{Ga}$ for proton reactions in natural zinc. Appl Radiat Isot 41:606-608. EXFOR: D0089 \#1

169. Tárkányi F, Szelecsényi F, Kovács Z, Sudar S (1990) Excitation functions of proton induced nuclear reactions on enriched ${ }^{66} \mathrm{Zn}$, ${ }^{67} \mathrm{Zn}$ and ${ }^{68} \mathrm{Zn}$ production of ${ }^{67} \mathrm{Ga}$ and ${ }^{66} \mathrm{Ga}$. Radiochim Acta 50:19-26. EXFOR: D4004

170. Hermanne A, Walravens N, Cicchelli O (192) Optimization of isotope production by cross section determination. In: Qaim SM (ed) International conference on nuclear data for science and technology, 13-17 May 1991, Jülich, Germany. Springer-Verlag, Berlin, Germany, pp 616-618. EXFOR: A0494

171. Nortier FM, Mills SJ, Steyn GF (1991) Excitation functions and yields of relevance to the production of ${ }^{67} \mathrm{Ga}$ by proton bombardment of ${ }^{\text {nat }} \mathrm{Zn}$ and ${ }^{\text {nat }} \mathrm{Ge}$ up to $100 \mathrm{MeV}$. Appl Radiat Isot 42:353-359. EXFOR: A0498

172. Szelecsényi F, Boothe TE, Tavano E, Plitnikas ME, Feijoo Y, Takács S, Tárkányi F, Szǔcs Z (1994) New cross section data for 66-67-68 $\mathrm{Zn}+\mathrm{p}$ reactions up to $26 \mathrm{MeV}$. Int. Conf. Nucl. Data for Science and Technology, Gatlinburg, Tennessee, USA (1994) 393. EXFOR: D40250

173. Hermanne A (1997) Evaluated cross section and thick target yield data of $\mathrm{Zn}+\mathrm{p}$ processes for practical applications (private communication). EXFOR: D4093

174. Szelecsényi F, Boothe TE, Takács S, Tárkányi F, Tavano E (1998) Evaluated cross section and thick target yield data bases of $\mathrm{Zn}+\mathrm{p}$ processes for practical applications. Appl Radiat Isot 49:1005-1032. EXFOR: C0506 
175. Szelecsényi F, Kovács Z, van der Walt TN, Steyn GF, Suzuki $\mathrm{K}$, Okada K (2003) Investigation of the ${ }^{\text {nat }} \mathrm{Zn}(\mathrm{p}, \mathrm{x})^{62} \mathrm{Zn}$ nuclear process up to $70 \mathrm{MeV}$ : a new $62 \mathrm{Zn} / 62 \mathrm{Cu}$ generator. Appl Radiat Isot 58:377-384. EXFOR: D4117

176. Szelecsényi F, Steyn GF, Kovács Z, van der Walt TN, Suzuki K, Okada K, Mukai K (2005) New cross-section data for the ${ }^{66} \mathrm{Zn}(\mathrm{p}$, n) ${ }^{66} \mathrm{Ga},{ }^{68} \mathrm{Zn}(\mathrm{p}, 3 \mathrm{n}){ }^{66} \mathrm{Ga}$, ${ }^{\text {nat }} \mathrm{Zn}(\mathrm{p}, \mathrm{x}){ }^{66} \mathrm{Ga},{ }^{68} \mathrm{Zn}(\mathrm{p}, 2 \mathrm{n}){ }^{67} \mathrm{Ga}$ and nat $\mathrm{Zn}(\mathrm{p}, \mathrm{x}){ }^{67} \mathrm{Ga}$ nuclear reactions up to $100 \mathrm{MeV}$. Nucl Instrum Methods Phys Res B 234:375-386. EXFOR: E1935

177. Al-Saleh FS, Al Mugren KS, Azzam A (2007) Excitation function measurements and integral yields estimation for ${ }^{\text {nat }} \mathrm{Zn}(\mathrm{p}$, $\mathrm{x})$ reactions, at low energies. Appl Radiat Isot 65:1101-1107. EXFOR: O1547

178. Porges KG (1956) Alpha excitation functions of silver and copper. Phys Rev 101:225-230. EXFOR: R0039

179. Porile NT, Morrison DL (1959) Reactions of $\mathrm{Cu}^{63}$ and $\mathrm{Cu}^{65}$ with alpha particles. Phys Rev 116:1193-1200. EXFOR: B0156

180. Bryant EA, Cochran DRF, Knight JD (1963) Excitation functions of reactions of 7 to $24 \mathrm{MeV} \mathrm{He}^{3}$ Ions with $\mathrm{Cu}^{63}$ and $\mathrm{Cu}^{65}$. Phys Rev 130:1512-1522. EXFOR: B0079

181. Stelson PH, McGowan FK (1964) Cross sections for $(\alpha, n)$ reactions for medium weight nuclei. Phys Rev 133:B911-B919. EXFOR: P0058

182. Zhukova OA, Kanasevich VI, Laptev SV, Chursin GP (1970) Excitation functions of $\alpha$-particle induced reactions on copper isotopes at energies up to $30 \mathrm{MeV}$. Izv Akad Nauk Kaz SSR Ser Fiz-Mat 4:1

183. Nassiff SJ, Nassiff W (1983) Cross sections and thick target yields of alpha-particle induced reactions. IAEA contract 2499/ R1/RB. EXFOR: D0046

184. Rizvi IA, Ansari MA, Gautam RP, Singh RKY, Chaubey AK (1987) Excitation functions studies of ( $\alpha$, xpyn) reactions for 63,65Cu and pre-equilibrium effect. J. Phys. Soc. Japan 56:31353144. EXFOR: D0090

185. Zweit J, Sharma H, Downey S (1987) Production of gallium-66, a short -lived positron emitting radionuclide. Appl Radiat Isot 38:499-501. EXFOR: D0119

186. Bhardwaj HD, Gautam AK, Prasad R (1988) Measurement and analysis of excitation functions for alpha-induced reactions in copper. Pramana J Phys 31:109-123. EXFOR: A0465

187. Mohan Rao AV, Mukherjee S, Rama Rao J (1991) Alpha particle induced reactions on copper and tantalum. Pramana J. Phys. $36: 115-123$

188. Bonesso O, Ozafran MJ, Mosca HO, Vazquez ME, Capurro OA, Nassiff SJ (1991) Study of pre-equilibrium effects on $\alpha$-induced reactions on copper. J Radioanal Nucl Chem 152:189-197. EXFOR: D0092

189. Tárkányi F, Szelecsényi F, Kopecký P (1992) Cross section data for proton, $\mathrm{He}$ and $\alpha$-particle induced reactions on ${ }^{\text {nat }} \mathrm{Ni}$, ${ }^{\text {nat }} \mathrm{Cu}$ and ${ }^{\text {nat }} \mathrm{Ti}$ for monitoring beam performance. In: Qaim SM (ed) International conference on nuclear data for science and technology, 13-17 May 1991, Jülich, Germany. Springer-Verlag, Berlin, pp 529-532. EXFOR: D4080

190. Singh NL, Patel BJ, Somayajulu DRS, Chintalapudi SN (1994) Analysis of the excitation functions of ( $\alpha$, xnyp) reactions on natural copper. Pramana J Phys 42:349-363. EXFOR: D0099

191. Tárkányi F, Szelecsényi F, Takács S, Hermanne A, Sonck M, Thielemans A, Mustafa MG, Shubin Y, Zhuang Y (2000) New experimental data, compilation and evaluation for the ${ }^{\text {nat }} \mathrm{Cu}(\alpha$, $\mathrm{x})^{66} \mathrm{Ga},{ }^{\text {nat }} \mathrm{Cu}(\alpha, \mathrm{x}){ }^{67} \mathrm{Ga}$ and ${ }^{\text {nat }} \mathrm{Cu}(\alpha, \mathrm{x}){ }^{65} \mathrm{Zn}$ monitor reactions. Nucl Instrum Methods Phys Res B 168:144-168. EXFOR: D4085

192. Szelecsényi F, Suzuki K, Kovács Z, Takei M, Okada K (2001) Alpha beam monitoring via ${ }^{\text {nat }} \mathrm{Cu}+$ alpha processes in the energy range from 40 to $60 \mathrm{MeV}$. Nucl Instrum Methods Phys Res B 184:589-596. EXFOR: E1996
193. Takács S, Tárkányi F, Hermanne A (2007) Cross section measurements of nuclear reactions on $\mathrm{Cu}$ and $\mathrm{Ti}$ target by alpha bombardment for monitoring use (private communication)

194. Adam-Rebeles R, Hermanne A, Van den Winkel P, Tárkányi F, Takács S, Daraban L (2008) Alpha induced reactions on ${ }^{114} \mathrm{Cd}$ and ${ }^{116} \mathrm{Cd}$ : An experimental study of excitation functions. Nucl Instrum Methods Phys Res B 266:4731-4737. EXFOR: D4204

195. Szelecsényi F, Kovács Z, Nagatsu K, Fukumura K, Suzuki K, Mukai K (2012) Investigation of direct production of ${ }^{68} \mathrm{Ga}$ with low energy multiparticle accelerator. Radiochim Acta 100:5-11. EXFOR: D4276

196. Shahid M, Kim K, Kim G, Zaman M, Nadeem M (2015) Measurement of excitation functions in alpha induced reactions on ${ }^{\text {nat }} \mathrm{Cu}$. Nucl Instrum Methods Phys Res B 358:160-167. EXFOR: D7016

197. Kotelnikova GV, Lovchikova GN, Sal`nikov OA, Simakov SP, Trufanov AM, Fetisov NI (1980) The investigation of neutron energy spectra for ${ }^{68} \mathrm{Zn}(\mathrm{p}, \mathrm{n}){ }^{68} \mathrm{Ga}$ reaction. Fiz-Energy Inst. Obninsk report no. 1141, 1. EXFOR: A0223

198. Esat MT, Spear RH, Zyskind JL, Shapiro MH, Fowler WA, Davidson JM (1981) Test of global Hauser-Feshbach calculations for proton-induced reactions on ${ }^{68} \mathrm{Zn}$. Phys Rev C 23:1822-1825. EXFOR: C0650

199. Vinogradov VM, Zhuravlev Y, Zarubin PP, Kolozhvari AA, Sergeev VO, Sitnikova IV (1993) Excitation functions of (p,n) reactions on zinc isotopes in the range of $E(p)$ from 4.9 to $5.9 \mathrm{MeV}$. Izv Rossiiskoi Akademii Nauk Ser Fiz 57(5):154. EXFOR: A0841

200. Zhuravlev Y, Zarubin PP, Zeic Yu V, Kolozhvari AA, Chelgunov IV (1995) Excitation functions of (p,n) reactions on nuclei of isotopes $\mathrm{Zn}$ from $E(p)=5.6$ To $6.8 \mathrm{MeV}$. Izv Rossiiskoi Akademii Nauk Ser Fiz 59:118. EXFOR: A0550

201. Mukherjee S, Kumar BB, Singh NL (1997) Excitation functions of alpha particle induced reactions on aluminium and copper. Pramana J Phys 49:253-261. EXFOR: O1180

202. Navin A, Tripathi V, Blumenfeld Y, Nanal V, Simenel C, Casandjian JM, de France G, Raabe R, Bazin D, Chatterjee A, Dasgupta M, Kailas S, Lemmon RC, Mahata K, Pillay RG, Pollacco EC, Ramachandran K, Rejmund M, Shrivastava A, Sida JL, Tryggestad E (2004) Direct and compound reactions induced by unstable helium beams near the Coulomb barrier. Phys Rev C 70:044601. EXFOR: D6021

203. Porile NT, Tanaka S, Amano H, Furukawa M, Iwata S, Yagi $\mathrm{M}$ (1963) Nuclear reactions of $\mathrm{Ga}^{69}$ and $\mathrm{Ga}^{71}$ with $13-56 \mathrm{MeV}$ protons. Nucl Phys 43:500-522. EXFOR: P0014

204. Adam-Rebeles R, Hermanne A, Van den Winkel P, De Vis L, Waegeneer R, Tárkányi F, Takács S, Takács MP (2013) ${ }^{68} \mathrm{Ge} /{ }^{68} \mathrm{Ga}$ production revisited: excitation curves, target preparation and chemical separation-purification. Radiochim Acta 101:481-489. EXFOR: D4321

205. Nozaki T, Itoh Y, Ogawa K (1979) Yield of ${ }^{73}$ Se for various reactions and its chemical processing. Int J Appl Radiat Isot 30:595-599

206. Mushtaq A, Qaim SM, Stocklin G (1988) Production of ${ }^{73}$ Se via $(\mathrm{p}, 3 \mathrm{n})$ and $(\mathrm{d}, 4 \mathrm{n})$ reactions on arsenic. Appl Radiat Isot 39:10851091. EXFOR: A0467

207. Fassbender M, de Villiers D, Nortier M, van der Walt N (2001) The ${ }^{\text {nat }} \operatorname{Br}(\mathrm{p}, \mathrm{x})^{73,75} \mathrm{Se}$ nuclear processes: a convenient route for the production of radioselenium tracers relevant to amino acid labelling. Appl Radiat Isot 54:905-913. EXFOR: D0168

208. de Villiers D, Nortier FM, Richter W (2002) Experimental and theoretical excitation functions for ${ }^{\text {nat }} \mathrm{Br}(\mathrm{p}, \mathrm{x})$ reactions. Appl Radiat Isot 57:907-913. EXFOR: O1022

209. Basile D, Birattari C, Bonardi M, Goetz L, Sabbioni E, Salomone A (1981) Excitation functions and production of arsenic 
radioisotopes for environmental toxicology and biomedical purposes. Int J Appl Radiat Isot 32:403-410. EXFOR: A0190

210. Horiguchi T, Kumahora H, Inoue H, Yoshizawa Y (1983) Excitation functions of $\mathrm{Ge}\left(\mathrm{p}\right.$, xnyp) reactions and production of ${ }^{68} \mathrm{Ge}$. Int J Appl Radiat Isot 34:1531-1535. EXFOR: E1968

211. Spahn I, Steyn GF, Nortier FM, Coenen HH, Qaim SM (2007) Excitation functions of ${ }^{\text {nat }} \mathrm{Ge}(\mathrm{p}, \mathrm{xn})^{71,72,73,74} \mathrm{As}$ reactions up to $100 \mathrm{MeV}$ with a focus on the production of ${ }^{72} \mathrm{As}$ for medical and ${ }^{73}$ As for environmental studies. Appl Radiat Isot 65:1057-1064. EXFOR: D0454

212. Takács S, Takács MP, Hermanne A, Tárkányi F, Adam-Rebeles R (2014) Excitation functions of longer lived radionuclides formed by deuteron irradiation of germanium. Nucl Instrum Methods Phys Res B 336:81-95. EXFOR: D4309

213. Brodovitch JC, Hogan JJ, Burns KI (1976) The pre-equilibrium statistical model: comparison of calculations with two (p, xn) reactions. J Inorg Nucl Chem 38:1581-1586. EXFOR: C2016

214. Qaim SM, Mushtaq A, Uhl M (1988) Isomeric cross-section ratio for the formation of ${ }^{73 \mathrm{~m}, \mathrm{~g}} \mathrm{Se}$ in various nuclear processes. Phys Rev C 38:645-650. EXFOR: O1041

215. Guillaume M, Lambrecht RM, Wolf AP (1978) Cyclotron isotopes and radiopharmaceuticals-XXVII. Se-73. Int J Appl Radiat Isot 29:411-417. EXFOR: B0152

216. Nozaki T, Iwamoto M, Itoh Y (1979) Production of ${ }^{77} \mathrm{Br}$ by various nuclear reactions. Int J Appl Radiat Isot 30:79-83. EXFOR: A0184

217. Paans AMJ, Welleweerd J, Vaalburg W, Reiffers S, Woldring MG (1980) Excitation functions for the production of bromine-75: A potential nuclide for the labelling of radiopharmaceuticals. Int $\mathbf{J}$ Appl Radiat Isot 31:267-273. EXFOR: A0253

218. Kovács Z, Blessing G, Qaim SM, Stocklin G (1985) Production of ${ }^{75} \mathrm{Br}$ via the ${ }^{76} \mathrm{Se}(\mathrm{p}, 2 \mathrm{n}){ }^{75} \mathrm{Br}$ reaction at a compact cyclotron. Int J Appl Radiat Isot 36:635-642. EXFOR: D0083

219. Hassan HE, Qaim SM, Shubin Yu, Azzam A, Morsy M, Coenen HH (2004) Experimental studies and nuclear model calculations on proton-induced reactions on ${ }^{\text {nat }} \mathrm{Se},{ }^{76} \mathrm{Se}$ and ${ }^{77} \mathrm{Se}$ with particular reference to the production of the medically interesting radionuclides ${ }^{76} \mathrm{Br}$ and ${ }^{77} \mathrm{Br}$. Appl Radiat Isot 60:899-909. EXFOR: D0164

220. Janssen AGM, Bosch RLP, de Goiej JJM, Theelen HMJ (1980) The reactions ${ }^{77} \mathrm{Se}(\mathrm{p}, \mathrm{n})$ and ${ }^{78} \mathrm{Se}(\mathrm{p}, 2 \mathrm{n})$ as production routes for ${ }^{77} \mathrm{Br}$. Int J Appl Radiat Isot 31:405-409. EXFOR: A0255

221. Spahn I, Steyn GF, Vermeulen C, Kovács Z, Szelecsényi F, Coenen HH, Qaim SM (2009) New cross section measurements for production of the positron emitters ${ }^{75} \mathrm{Br}$ and ${ }^{76} \mathrm{Br}$ via intermediate energy proton induced reactions. Radiochim Acta 97:535-541. EXFOR: D0568

222. Alfassi ZB, Weinreich R (1982) The production of positron emitters ${ }^{75} \mathrm{Br}$ and ${ }^{76} \mathrm{Br}$ : Excitation functions and yields for ${ }^{3} \mathrm{He}$ and $\alpha$-particle induced nuclear reactions on arsenic. Radiochim Acta 30:67-71. EXFOR: A0232

223. Hermanne A, Sonck M, Van Hoyweghen J, Terriere D, Mertens J (1994) Optimisation of radiobromine production from As-based targets through cross section determination. In: International conference on nuclear data for science and technology, Gatlinburg, Tennessee, USA, p 1039

224. Breunig K, Spahn I, Hermanne A, Spellerberg S, Scholten B, Coenen HH (2017) Cross section measurements of ${ }^{75} \mathrm{As}(\alpha$, $\mathrm{xn})^{76,77,78} \mathrm{Br}$ and ${ }^{75} \mathrm{As}(\alpha, \mathrm{x}){ }^{74} \mathrm{As}$ nuclear reactions using the monitor radionuclides ${ }^{67} \mathrm{Ga}$ and ${ }^{66} \mathrm{Ga}$ for beam evaluation. Radiochim Acta 105:431-439

225. Horiguchi T, Noma H, Yoshizawa Y, Takemi H, Hasai H, Kiso Y (1980) Excitation functions of proton induced nuclear reactions on ${ }^{85} \mathrm{Rb}$. Int J Appl Radiat Isot 31:141-151. EXFOR: B0111

226. Deptula C, Khalkin VA, Kim SH, Knotek O, Konov VA, Mikecz P, Poponenkova LM, Rurarz E, Zaitseva NG (1990) Excitation functions and yields for medically generator $\mathrm{Sr}^{82}-\mathrm{Rb}^{82}, \mathrm{Xe}^{123}$ $\mathrm{I}^{123}$ and $\mathrm{Bi}^{201}-\mathrm{Pb}^{201}-\mathrm{Tl}^{201}$ obtained with $100 \mathrm{MeV}$ protons. Nukleonika 35:3-47. EXFOR: O0306

227. Lagunas-Solar MC (1992) Radionuclide production with $>70-\mathrm{MeV}$ proton accelerators: current and future prospects. Nucl Instrum Methods Phys Res B 69:452-462. EXFOR: C0780

228. Gilabert E, Lavielle B, Neumann S, Gloris M, Michel R, Schiekel Th, Sudbrock F, Herpers U (1998) Cross sections for the protoninduced production of krypton isotopes from $\mathrm{Rb}, \mathrm{Sr}, \mathrm{Y}$, and $\mathrm{Zr}$ for energies up to $1600 \mathrm{MeV}$. Nucl Instrum Methods Phys Res B 145:293-319. EXFOR: O0505

229. Ido T, Hermanne A, Ditrói F, Szǔcs Z, Mahunka I, Tárkányi F (2002) Excitation functions of proton induced nuclear reactions on ${ }^{\text {nat }} \mathrm{Rb}$ from 30 to $70 \mathrm{MeV}$. Implication for the production of ${ }^{82} \mathrm{Sr}$ and other medically important $\mathrm{Rb}$ and $\mathrm{Sr}$ radioisotopes. Nucl Instrum Methods Phys Res B194:369-388. EXFOR: D4114

230. Kastleiner S, Qaim SM, Nortier FM, Blessing G, van der Walt TN, Coenen HH (2002) Excitation functions of ${ }^{85} \mathrm{Rb}(\mathrm{p}, \mathrm{xn}){ }^{85 \mathrm{~m}, \mathrm{~g}, 83,82,81} \mathrm{Sr}$ reactions up to $100 \mathrm{MeV}$ : integral tests of cross section data,comparison of production routes of ${ }^{83} \mathrm{Sr}$ and thick target yield of ${ }^{82} \mathrm{Sr}$. Appl Radiat Isot 56:685-695. EXFOR: D4127

231. Kovács Z, Tárkányi F, Qaim SM, Stocklin G (1991) Excitation functions for the formation of some radioisotopes of rubidium in proton induced nuclear reactions on ${ }^{\text {nat }} \mathrm{Kr},{ }^{82} \mathrm{Kr}$ and ${ }^{83} \mathrm{Kr}$ with special reference to the production of ${ }^{81} \mathrm{Rb}\left({ }^{81 \mathrm{~m}} \mathrm{Kr}\right)$ generator radionuclide. Appl Radiat Isot 42:329-335. EXFOR: A0489

232. Steyn GF, Mills SJ, Nortier FM, Haasbroek FJ (1991) Integral excitation functions for (nat) $\mathrm{Kr}+\mathrm{p}$ up to $116 \mathrm{MeV}$ and optimization of the production of ${ }^{81} \mathrm{Rb}$ for ${ }^{81 \mathrm{~m}} \mathrm{Kr}$ generators. Appl Radiat Isot 42:361-370. EXFOR: A0499

233. Ditrói F, Tárkányi F, Takács S, Doczi R, Hermanne A, Ignatyuk AV (2012) Study of excitation function of deuteron induced reactions on ${ }^{\text {nat }} \mathrm{Kr}$ up to $20 \mathrm{MeV}$. Appl Radiat Isot 70:574-582. EXFOR: D4262

234. Delaunay-Olkowsky J, Strohal P, Cindro N (1963) Total reaction cross section of proton induced reactions. Nucl Phys 47:266-272. EXFOR: O2103

235. Rösch F, Qaim SM, Stocklin G (1993) Nuclear data relevant to the production of the positron emitting radioisotope ${ }^{86} \mathrm{Y}$ via the ${ }^{86} \mathrm{Sr}(\mathrm{p}, \mathrm{n})$ - and ${ }^{\mathrm{nat}} \mathrm{Rb}\left({ }^{3} \mathrm{He}, \mathrm{xn}\right)$-processes. Radiochim Acta 61:1-8. EXFOR: D4030

236. Sachdev DR, Porile NT, Yaffe L (1967) Reactions of ${ }^{88} \mathrm{Sr}$ with protons of energies 7-85 MeV. Can J Chem 45:1149-1160. EXFOR: B0069

237. Iwata SJ (1962) Isomeric cross section ratios in alpha-particle reactions. J Phys Soc Jpn 17:1323-1333. EXFOR: P0064

238. Demeyer A, Chevarier N, Chevarier A, Tran MD (1971) Reculs moyens, rapports isomeriques et fonctions d'excitation pour les reactions induites par des particules alpha sur le rubidium. J de Physique 32:583-593. EXFOR: O2038

239. Guin R, Das SK, Saha SK (2000) Cross-sections and linear momentum transfer in $\alpha$-induced reactions on ${ }^{85} \mathrm{Rb}$. Radiochim Acta 88:435-438. EXFOR: A0111

240. Agarwal A, Bhardwaj MK, Rizvi IA, Chaubey AK (2003) Measurement and analysis of excitation function for alpha induced reactions with rubidium. Indian J Pure Appl Phys 41:829-832. EXFOR: D0122

241. Albert RD (1959) (p, n) cross section and proton opticalmodel parameters in the 4 to $5.5 \mathrm{MeV}$ energy region. Phys Rev 115:925-927. EXFOR: T01300

242. Saha GB, Porile NT, Yaffe L (1966) (p, xn) and (p, pxn) reactions of yttrium-89 with 5-85-MeV protons. Phys Rev 144:962-971. EXFOR: T0195 
243. Johnson CH, Kernell RL, Ramavataram S (1968) The ${ }^{89} Y(p$, n) ${ }^{89} \mathrm{Zr}$ cross section near the first two analogue resonances. Nucl Phys A 107:21-34. EXFOR: C0774

244. Birattari C, Gadioli E, Gadioli Erba E, Grassi Strini AM, Strini G, Tagliaferri G (1973) Pre-equilibrium processes in (p, n) reactions. Nucl Phys A 201:579-592. EXFOR: B0018

245. Mustafa MG, West HI Jr, O’Brien H, Lanier RG, Benhamou M, Tamura T (1988) Measurements and a direct-reaction-plusHauser-Feshbach analysis of ${ }^{89} \mathrm{Y}(\mathrm{p}, \mathrm{n}){ }^{89} \mathrm{Zr},{ }^{89} \mathrm{Y}(\mathrm{p}, 2 \mathrm{n}){ }^{88} \mathrm{Zr}$, and ${ }^{89} \mathrm{Y}(\mathrm{p}, \mathrm{pn}){ }^{88} \mathrm{Y}$ reactions up to $40 \mathrm{MeV}$. Phys Rev C $38: 1624$ 1637. EXFOR: A0403

246. Wenrong Zhao, Shen Qingbiao Lu, Weixiang Hanlin, Yu (1992) Investigation of ${ }^{89} \mathrm{Y}(\mathrm{p}, \mathrm{n}){ }^{89} \mathrm{Zr},{ }^{89} \mathrm{Y}(\mathrm{p}, 2 \mathrm{n}){ }^{88} \mathrm{Zr}$ and ${ }^{89} \mathrm{Y}(\mathrm{p}, \mathrm{pn})^{88} \mathrm{Y}$ reactions up to $22 \mathrm{MeV}$. Chin J Nucl Phys (Beijing) 14:7. EXFOR: S0040

247. Uddin MS, Hagiwara M, Baba M, Tárkányi F, Ditrói F (2005) Experimental studies on excitation functions of the protoninduced activation reactions on yttrium. Appl Radiat Isot 63:367-374. EXFOR: E1934

248. Omara HM, Hassan KF, Kandil SA, Hegazy FE, Saleh ZA (2009) Proton induced reactions on ${ }^{89} \mathrm{Y}$ with particular reference to the production of the medically interesting radionuclide ${ }^{89} \mathrm{Zr}$. Radiochim Acta 97:467-471. EXFOR: D0584

249. Steyn GF, Vermeulen C, Szelecsényi F, Kovács Z, Suzuki K, Fukumura T, Nagatsu K (2011) Excitation functions of proton induced reactions on ${ }^{89} \mathrm{Y}$ and ${ }^{93} \mathrm{Nb}$ with emphasis on the production of selected radio-zirconiums. J. Korean Phys. Soc. 59:1991-1994. EXFOR: D0629

250. Satheesh B, Musthafa MM, Singh BP, Prasad R (2011) Nuclear isomers ${ }^{90 \mathrm{~m}, \mathrm{~g}} \mathrm{Zr},{ }^{89 \mathrm{~m}, \mathrm{~g}} \mathrm{Y}$ and ${ }^{85 \mathrm{~m}, \mathrm{~g}} \mathrm{Sr}$ formed by bombardment of ${ }^{89} \mathrm{Y}$ with proton beams of energies from 4 to $40 \mathrm{MeV}$. Int J Mod Phys E 20:2119-2132. EXFOR: D6178

251. Khandaker MU, Kim K, Lee M-W, Kim K-S, Kim G, Otuka $\mathrm{N}$ (2012) Investigations of ${ }^{89} \mathrm{Y}(\mathrm{p}, \mathrm{x})^{86,88,89 \mathrm{~g}} \mathrm{Zr},{ }^{86 \mathrm{~m}+\mathrm{g}, 87 \mathrm{~g}, 87 \mathrm{~m}, 88 \mathrm{~g}} \mathrm{Y}$, ${ }^{85 \mathrm{~g}} \mathrm{Sr}$, and ${ }^{84 \mathrm{~g}} \mathrm{Rb}$ nuclear processes up to $42 \mathrm{MeV}$. Nucl Instrum Methods Phys Res B 271:72-81. EXFOR: D0675

252. Baron N, Cohen BL (1963) Activation cross-section survey of deuteron-induced reactions. Phys Rev 129:2636-2642. EXFOR: D4059

253. La Gamma AM, Nassiff SJ (1973) Excitation functions for deuteron-induced reactions on ${ }^{89}$ Y. Radiochim Acta 19:161-162. EXFOR: F0843

254. Bissem HH, Georgi R, Scobel W, Ernst J, Kaba M, Rama Rao J, Strohe H (1980) Entrance and exit channel phenomena in d- and ${ }^{3}$ He-induced pre-equilibrium decay. Phys Rev C 22:1468-1484. EXFOR: A0347

255. Degering D, Unterricker S, Stolz W (1988) Excitation function of the ${ }^{89} \mathrm{Y}(\mathrm{d}, 2 \mathrm{n})^{89} \mathrm{Zr}$ reaction. J Radioanal Nucl Chem Lett 127:7-11. EXFOR: O1107

256. West HI, Brien HO, Lanier RG, Nagle RJ, Mustafa MG (1993) Measurements of the excitation functions of the isobaric chain ${ }^{87} \mathrm{Y},{ }^{87 \mathrm{~m}} \mathrm{Y},{ }^{87 \mathrm{~g}} \mathrm{Y}$, and ${ }^{87 \mathrm{~m}} \mathrm{Sr}$. Some excitation functions of proton and deuteron induced reactions on ${ }^{89} \mathrm{Y}$. University of California Lawrence Radiation Laboratory report, vol 5, no.115738, p 1. EXFOR: C1159

257. Uddin MS, Baba M, Hagiwara M, Tárkányi F, Ditrói F (2007) Experimental determination of deuteron-induced activation cross sections of yttrium. Radiochim Acta 95:187-192. EXFOR: E2051

258. Lebeda O, Štursa J, Ráliš J (2015) Experimental cross-sections of deuteron-induced reaction on ${ }^{89} \mathrm{Y}$ up to $20 \mathrm{MeV}$; comparison of ${ }^{\text {nat }} \mathrm{Ti}(\mathrm{d}, \mathrm{x}) 48 \mathrm{~V}$ and ${ }^{27} \mathrm{Al}(\mathrm{d}, \mathrm{x})^{24} \mathrm{Na}$ monitor reactions. Nucl Instrum Methods Phys Res B 360:118-128. EXFOR: D0777

259. Ditrói F, Takács S, Tárkányi F, Baba M, Corniani E, Shubin YuN (2008) Study of proton induced reactions on niobium targets up to $70 \mathrm{MeV}$. Nucl Instrum Methods Phys Res B 266:5087-5100. EXFOR: D4212 \#1

260. Ditrói F, Hermanne A, Corniani E, Takács S, Tárkányi F, Csikái J, Shubin YuN (2009) Investigation of proton induced reactions on niobium at low and medium energies. Nucl Instrum Methods Phys Res B 267:3364-3374. EXFOR: D4228 \#1

261. Titarenko YE, Batyaev VF, Titarenko AY, Butko MA, Pavlov KV, Florya SN, Tikhonov RS, Zhivun VM, Ignatyuk AV, Mashnik SG, Leray S, Boudard A, Cugnon J, Mancusi D, Yariv Y, Nishihara K, Matsuda N, Kumawat H, Mank G, Gudowski W (2011) Measurement and simulation of the cross sections for nuclide production in $\mathrm{Nb}-93$ and $\mathrm{Ni}$-nat targets irradiated with $0.04-$ to $2.6-\mathrm{GeV}$ protons. Yadernaya Fizika 74:561-573. EXFOR: A0906

262. Kim GN (2016) Unpublished information (private communication)

263. Smend F, Weirauch W, Schmidt-Ott W-D, Flammersfeld A (1967) Wirkungsquerschnitte und Isomerenverhaltnis fur die Reaktionen $89-\mathrm{Y}(\alpha, 3 \mathrm{n}) 90 \mathrm{~g}-\mathrm{Nb}$ und $89-\mathrm{Y}(\alpha, 3 \mathrm{n}) 90 \mathrm{~m}-\mathrm{Nb}$. Z Phys 207:28-34. EXFOR: O1366

264. Mukherjee S, Kumar BB, Rashid MH, Chintalapudi SN (1997) $\alpha$-particle induced reactions on yttrium and terbium. Phys Rev C 55:2556-2562. EXFOR: O1114

265. Chaubey AK, Rizvi IA (1999) Non-equilibrium effects in alpha induced reactions in some natural elements. Indian J Pure Appl Phys 37:791-793. EXFOR: D6217

266. Singh NL, Gadkari MS, Chintalapudi SN (2000) Measurement and analysis of alpha particle induced reactions on yttrium. Phys Scr 61:550-554. EXFOR: D0067

267. Shahid M, Kim K, Naik H, Zaman M, Kim G, Yang S-C, Song $\mathrm{T}-\mathrm{Y}$ (2015) Measurement of excitation functions in alphainduced reactions on yttrium. Nucl Instrum Methods Phys Res B 342:158-165. EXFOR: D7014

268. Graf HP, Münzel H (1974) Excitation functions for $\alpha$-particle reactions with molybdenum isotopes. J Inorg Nucl Chem 36:3647-3657. EXFOR: B0040

269. Denzler F-O, Rosch F, Qaim SM (1995) Excitation functions of $\alpha$-particle induced nuclear reactions on highly enriched ${ }^{92} \mathrm{Mo}$ : comparative evaluation of production routes for ${ }^{94 \mathrm{~m}} \mathrm{Tc}$. Radiochim Acta 68:13-20. EXFOR: D4016

270. Ditrói F, Hermanne A, Tárkányi F, Takács S, Ignatyuk AV (2012) Investigation of the $\alpha$-particle induced nuclear reactions on natural molybdenum. Nucl Instrum Methods Phys Res B 285:125141. EXFOR: D4269

271. Skakun EA, Batij VG, Rakivnenko YuN, Rastrepin OA (1987) Excitation functions and isomer ratios for up-to-9-MeV proton interactions with $\mathrm{Zr}$ and Mo isotope nuclei. Yad Fiz 46:28-39. EXFOR: A0338

272. Rosch F, Qaim SM (1993) Nuclear data relevant to the production of the positron emitting technetium isotope ${ }^{94 \mathrm{~m}} \mathrm{Tc}$ via the ${ }^{94} \mathrm{Mo}(\mathrm{p}, \mathrm{n})$-reaction. Radiochim Acta 62:115-121. EXFOR: D4021

273. Zhuravlev Y, Zarubin PP, Kolozhvari AA (1994) Excitation functions of $(\mathrm{p}, \mathrm{n})$ reaction on the Mo isotope nuclei in the energy interval from thresholds to 7.2 MeV. Izv. Rossiiskoi Akademii Nauk Ser Fiz 58:106. EXFOR: A0575

274. Lebeda O, Pruszynski M (2010) New measurement of excitation functions for $(\mathrm{p}, \mathrm{x})$ reactions on ${ }^{\text {nat }} \mathrm{Mo}$ with special regard to the formation of ${ }^{95 \mathrm{~m}} \mathrm{Tc},{ }^{96 \mathrm{~m}+\mathrm{g}} \mathrm{Tc},{ }^{99 \mathrm{~m}} \mathrm{Tc}$ and ${ }^{99} \mathrm{Mo}$. Appl Radiat Isot 68:2355-2365. EXFOR: D0615

275. Cervenák J, Lebeda O (2016) Experimental cross-sections for proton-induced nuclear reactions on ${ }^{\text {nat }}$ Mo. Nucl Instrum Methods Phys Res B 380:32-49. EXFOR: D0805

276. Nortier FM, Mills SJ, Steyn GF (1990) Excitation functions and production rates of relevance to the production of ${ }^{111}$ In by proton 
bombardment of ${ }^{\text {nat }} \mathrm{Cd}$ and ${ }^{\text {nat }}$ In up to $100 \mathrm{MeV}$. Appl Radiat Isot 41:1201-1208. EXFOR: A0500

277. Lundqvist H, Scott-Robson S, Einarsson L, Maimborg P (1991) ${ }^{110} \mathrm{Sn} /{ }^{110} \mathrm{In}$ - a new generator system for positron emission tomography. Appl Radiat Isot 42:447-450

278. Tárkányi F, Takács S, Ditrói F, Hermanne A, Baba M, Mohsena BMA, Ignatyuk AV (2015) New cross section data and review of production routes of medically used ${ }^{110 \mathrm{~m}}$ In. Nucl Instrum Methods Phys Res B 351:6-15. EXFOR: D4327

279. Tárkányi F, Ditrói F, Hermanne A, Takács S, Baba M (2016) Investigation of activation cross sections of proton induced reactions on indium up to $70 \mathrm{MeV}$ for practical applications. Appl Radiat Isot 107:391-400. EXFOR: D4333

280. Hermanne A, Daraban L, Adam-Rebeles R, Ignatyuk A, Tárkányi F, Takács S (2010) Alpha induced reactions on ${ }^{\text {nat }} \mathrm{Cd}$ up to 38.5 MeV Experimental and theoretical studies of the excitation function. Nucl Instrum Methods Phys Res B268:1376-1391. EXFOR: D4231001

281. Khandaker MU, Kim K, Lee MW, Kim GN (2014) Investigation of activation cross-sections of alpha-induced nuclear reactions on natural cadmium. Nucl Instrum Methods Phys Res B 333:80-91. EXFOR: D7018

282. Duchemin C, Essayan M, Guertin A, Haddad F, Michel N, Metivier V (2016) How to produce high specific activity tin117m using alpha particle beam. Appl Radiat Isot 115:113-124. EXFOR: O2314

283. Ditrói F, Takács S, Haba H, Komori Y, Aikawa M (2016) Cross section measurement of alpha particle induced nuclear reactions on natural cadmium up to $52 \mathrm{MeV}$. Appl Radiat Isot 118:266276. EXFOR: D4359

284. Otozai K, Kume S, Mito A, Okamura H, Tsujino R, Kanchiku Y, Katoh T, Gotoh H (1966) Excitation functions for the reactions induced by protons on $\mathrm{Cd}$ up to $37 \mathrm{MeV}$. Nucl. Phys. 80:335348. EXFOR: P0019

285. Skakun EA, Kljucharev AP, Rakivnenko YN, Romanij A (1975) Excitation functions of (p,n)- and (p,2n)-reactions on cadmium isotopes. Izv Akademii Nauk SSSR Ser Fiz 39:24-30. EXFOR: A0001

286. Kormali SM, Swindle DL, Schweikert EA (1976) Charged particle activation of medium Z-elements. II. Proton excitation functions. J Radioanal Chem 31:437-450. EXFOR: D4073

287. Tárkányi F, Király B, Ditrói F, Takács S, Csikái J, Hermanne A, Uddin MS, Hagiwara M, Baba M, Ido T, Shubin YuN, Kovalev SF (2006) Activation cross-sections on cadmium: proton induced nuclear reactions up to $80 \mathrm{MeV}$. Nucl Instrum Methods Phys Res B 245:379-394. EXFOR: D4170

288. Al-Saleh FS (2008) Cross sections of proton induced nuclear reactions on natural cadmium leading to the formation of radionuclides of indium. Radiochim Acta 96:461-465. EXFOR: D0467

289. Khandaker MU, Kim K, Lee MW, Kim KS, Kim GN, Cho YS, Lee YO (2008) Production cross-sections for the residual radionuclides from the ${ }^{\text {nat }} \mathrm{Cd}(\mathrm{p}, \mathrm{x})$ nuclear processes. Nucl Instrum Methods Phys Res B 266:4877-4887. EXFOR: D0516

290. Usher OH, Maceiras E, Saravi MC, Nassiff SJ (1977) Production cross section and isomeric ratios for the isomeric pair In$110 \mathrm{~m} / \mathrm{In}-110 \mathrm{~g}$ formed in $\mathrm{Cd}(\mathrm{d}, \mathrm{xn})$ reactions. Radiochim Acta 24:55-57. EXFOR: D4064001

291. Tárkányi F, Király B, Ditrói F, Takács S, Csikái J, Hermanne A, Uddin MS, Hagiwara M, Baba M, Ido T, Shubin YuN, Kovalev SF (2007) Activation cross sections on cadmium: Deuteron induced nuclear reactions up to $40 \mathrm{MeV}$. Nucl Instrum Methods Phys Res B 259:817-828. EXFOR: D4179001

292. Mukhammedov S, Pardayev E (1958) The excitation functions of $(\mathrm{d}, 2 \mathrm{n})$ type nuclear reactions on the isotopes of cadmium and lead. Izv Akad Nauk Uzb SSR Ser Fiz-Mat 5:81. EXFOR: D0727
293. Fukushima S, Hayashi S, Kume S, Okamura H, Otozai K, Sakamoto K, Yoshizawa Y (1963) Excitation functions for the reactions induced by alpha particles on ${ }^{107} \mathrm{Ag}$. Nucl Phys 41:275-290. EXFOR: E1874

294. Misaelides P, Münzel H (1980) Excitation functions for ${ }^{3} \mathrm{He}$ - and $\alpha$-induced reactions with ${ }^{107} \mathrm{Ag}$ and ${ }^{109} \mathrm{Ag}$. J Inorg Nucl Chem 42:937-948. EXFOR: A0319

295. Wasilevsky C, De la Vega Vedoya M, Nassiff SJ (1985) Isomer yield ratios and cross sections for ${ }^{110} \operatorname{In}(4.9 \mathrm{~h}) /{ }^{110} \operatorname{In}(69 \mathrm{~min})$ and ${ }^{108} \operatorname{In}(58 \mathrm{~min}) /{ }^{108} \operatorname{In}(39.6 \mathrm{~min})$ produced by alpha reactions on silver. J Radioanal Nucl Chem 95:29-44. EXFOR: A0314

296. Chaubey AK, Bhardwaj MK, Gautam RP, Singh RKY, Afzal Ansari M, Rizvi IA, Singh H (1990) Pre-equilibrium decay process in the alpha induced reactions of silver isotopes. Appl Radiat Isot 41:401-405. EXFOR: D6208

297. Patel HB, Gadkari MS, Dave B, Singh NL, Mukherjee S (1996) Analysis of the excitation function of alpha-particle-induced reactions on natural silver. Can J Phys 74:618-625. EXFOR: D0065

298. Takács S, Hermanne A, Tárkányi F, Ignatyuk A (2010) Crosssections for alpha particle produced radionuclides on natural silver. Nucl Instrum Methods Phys Res B 268:2-12. EXFOR: D4217

299. Yalçin C, Gyurky G, Rauscher T, Kiss GG, Őzkan N, Güray RT, Halasz Z, Szǔcs T, Fülöp Z, Farkas J, Korkulu Z, Somorjai E (2015) Test of statistical model cross section calculations for $\alpha$-induced reactions on ${ }^{107} \mathrm{Ag}$ at energies of astrophysical interest. Phys Rev C 91:034610. EXFOR: D4328

300. Adam-Rebeles R, Hermanne A, Takács S, Tárkányi F, Kovalev SF, Ignatyuk A (2007) Alpha induced reactions on ${ }^{\text {nat }} \mathrm{Sn}$ : An experimental study of excitation functions and possible production pathways. Nucl Instrum Methods Phys Res B 260:672-684. EXFOR: D4189

301. Filipescu D, Avrigeanu V, Glodariu T, Mihai C, Bucurescu D, Ivascu M, Cata-Danil I, Stroe L, Sima O, Cata-Danil G, Deleanu D, Ghita DG, Marginean N, Marginean R, Negret A, Pascu S, Sava T, Suliman G, Zamfir NV (2011) Cross sections for $\alpha$-particle induced reactions on ${ }^{115,116} \mathrm{Sn}$ around the Coulomb barrier. Phys Rev C 83:064609. EXFOR: D0652

302. Batij VG, Baskova KA, Kuz`menko VA, Makuni BM, Rastrepin OA, Skakun EA, Chugaj TV, Shavtvalov LJ (1984) Excitation functions of ${ }^{116,117} \mathrm{Sn}(\alpha, \mathrm{xn})$ reactions in the energy range under $30 \mathrm{MeV}$. IN: 34th Conference nuclear spectroscopy and nuclear structure. Alma-Ata, USSR, p 355. EXFOR: A0217

303. Yi JH, Miller DA (1992) Cross section of natSb(p, x) reactions for 30-46 MeV. Appl Radiat Isot 43:1103-1106. EXFOR: C0092

304. Lagunas-Solar MC, Haff RP, Carvacho OF, Yang ST, Yano Y (1990) Cyclotron production of PET radionuclides: 118Sb (3.5 $\min$; beta $+75 \%$; EC $25 \%$ ) from high-energy protons on natural Sb targets. Appl Radiat Isot 41:521-529. EXFOR: C0094

305. Takács S, Takács MP, Hermanne A, Tarkányi F, Adam-Rebeles R (2013) Cross sections of proton induced reactions on ${ }^{\text {nat }} \mathrm{Sb}$. Nucl Instrum Methods Phys Res B 297:44-57. EXFOR: D4279

306. Takács S, Takács MP, Hermanne A, Tárkányi F, Adam-Rebeles $R$ (2012) Cross sections of deuteron-induced reactions on ${ }^{\text {nat }} \mathrm{Sb}$ up to $50 \mathrm{MeV}$. Nucl Instrum Methods Phys Res B 278:93-105. EXFOR: D4265

307. Hohn A, Coenen HH, Qaim SM (1998) Nuclear data relevant to the production of ${ }^{120 \mathrm{~g}} \mathrm{I}$ via the ${ }^{120} \mathrm{Te}(\mathrm{p}, \mathrm{n})$-process at a small-sized cyclotron. Appl Radiat Isot 49:1493-1496. EXFOR: D4121

308. El-Azony K, Suzuki K, Fukumura T, Szelecsényi F, Kovács $\mathrm{Z}$ (2008) Proton induced reactions on natural tellurium up to $63 \mathrm{MeV}$ : data validation and investigation of possibility of ${ }^{124} \mathrm{I}$ production. Radiochim Acta 96:763-769. EXFOR: D0502

309. Güray RT, Őzkan N, Yalçin C, Palumbo A, deBoer R, Görres J, Leblanc PJ, O’Brien S, Strandberg E, Tan WP, Wiescher M, 
Fülöp Z, Somorjai E, Lee HY, Greene JP (2009) Measurements of proton-induced reaction cross sections on ${ }^{120} \mathrm{Te}$ for the astrophysical p process. Phys Rev C 80:035804. EXFOR: C1742

310. Ahmed AM, Hassan HE, Hassan KF, Khalaf AM, Saleh ZA (2011) Cross sections for the formation of radioiodines in proton bombardment of natural tellurium with particular reference to the validation of data for the production of ${ }^{123} \mathrm{I}$. Radiochim Acta 99:317-323. EXFOR: D0647

311. Tárkányi F, Qaim SM, Stocklin G, Sajjad M, Lambrecht RM (1991) Nuclear reaction cross sections relevant to the production of the ${ }^{122} \mathrm{Xe} \rightarrow{ }^{122}$ I generator system using highly enriched ${ }^{124} \mathrm{Xe}$ and a medium-sized cyclotron. Appl Radiat Isot 42:229-233. EXFOR: A0487

312. Hermanne A, Tárkányi F, Takács S, Adam-Rebeles R, Ignatyuk A, Spellerberg S, Schweikert R (2011) Limitation of the longlived ${ }^{121} \mathrm{Te}$ contaminant in production of ${ }^{123} \mathrm{I}$ through the ${ }^{124} \mathrm{Xe}(\mathrm{p}, \mathrm{x})$ route. Appl Radiat Isot 69:358-368. EXFOR: D4238

313. Diksic M, Yaffe L (1977) A study of ${ }^{127} I(p, x n)$ and ${ }^{127} I(p, p x n)$ reactions with special emphasis on production of ${ }^{123} \mathrm{Xe}$. J Inorg Nucl Chem 39:1299-1302. EXFOR: B0081

314. Lundqvist H, Malmborg P, Langstrom B, Suparb Na Chiengmai (1979) Simple production of ${ }^{77} \mathrm{Br}$ - and ${ }^{123} \mathrm{I}$ - and their use in the labelling of $\left[{ }^{77} \mathrm{Br}\right] \mathrm{BrUdR}$ and $\left[{ }^{123} \mathrm{I}\right] \mathrm{IUdR}$. Appl Radiat Isot 30:39-43. EXFOR: D0116 \#1

315. Lagunas-Solar MC, Carvacho OF, Liu Bo-Li, Jin Y, Sun Zhao Xiang (1986) Cyclotron production of high-purity ${ }^{123}$ I. A revision of excitation functions, thin-target and cumulative yields for ${ }^{127} \mathrm{I}(\mathrm{p}, \mathrm{xn})$ reactions. Appl Radiat Isot 37:823-833. EXFOR: A0363

316. Weinreich R, Schult O, Stocklin G (1974) Production of ${ }^{123}$ I via the ${ }^{123} \mathrm{Xe}(\beta+, \mathrm{EC}){ }^{123} \mathrm{I}$ process. Int J Appl Radiat Isot 25:535543. EXFOR: B0143

317. Husson JP, Legoux Y, Liang CF (1978) Study of the rate of formation of short-lived iodine and xenon isotopes at the synchrocyclotron of Orsay for use in medicine. Inst Phys Nucl Orsay 27(78):1-11. EXFOR: A0345

318. Fink RW, Wiig EO (1954) Reactions of cesium with protons at 60, 80, 100, 150, and $240 \mathrm{MeV}$. Phys Rev 96:185-187. EXFOR: $\mathrm{C} 2000$

319. Lagunas-Solar MC, Little FE, Moore HA Jr. (1982) Cyclotron production of ${ }^{128} \mathrm{Cs}$ (3.62 min). A new positron-emitting radionuclide for medical applications. Appl. Radiat. Isot 33:619-628. EXFOR: A0320

320. Deptula C, Kim SH, Knotek O, Mikolajewski S, Popinenkova LM, Rurarz E, Zaitseva NG (1990) Production of ${ }^{128,131} \mathrm{Ba},{ }^{132} \mathrm{Cs}$ in proton induced reactions on a Cs target and of ${ }^{127,129} \mathrm{Cs}$ in 3,4He induced reactions on I target. Nukleonika 35:63-77. EXFOR: A0572

321. Tárkányi F, Hermanne A, Takács S, Ditrói F, Király B, Yamazaki H, Baba M, Mohammadi A, Ignatyuk AV (2010) New measurements and evaluation of excitation functions for (p, xn), (p, pxn) and (p,2pxn) reactions on ${ }^{133} \mathrm{Cs}$ up to $70 \mathrm{MeV}$ proton energy. Appl Radiat Isot 68:47-58. EXFOR: D4226

322. Hogan JJ (1971) Study of the ${ }^{141} \operatorname{Pr}(\mathrm{p}, \mathrm{xn})$ reaction from $10-85$ MeV. J Inorg Nucl Chem 33:3627-3641. EXFOR: C0321

323. Zeisler S, Becker D (1999) Production of the ${ }^{140} \mathrm{Nd}:{ }^{140} \mathrm{Pr}$ radionuclide generator for biomedical studies. Journal of Labelled Compounds and Radiopharmaceuticals 42(Suppl. 1):S921-923 (abstracts from 13th Int. Symp. Radiopharm. Chem., 27 June-1 July 1999, St. Louis, Missouri, USA)

324. Hilgers K, Shubin YuN, Coenen HH, Qaim SM (2005) Experimental measurements and nuclear model calculations on the excitation functions of ${ }^{\text {nat }} \mathrm{Ce}\left({ }^{3} \mathrm{He}, \mathrm{xn}\right)$ and ${ }^{141} \mathrm{Pr}(\mathrm{p}, \mathrm{xn})$ reactions with special reference to production of the therapeutic radionuclide ${ }^{140} \mathrm{Nd}$. Radiochim Acta 93:553-560. EXFOR: O1352

325. Hermanne A, Tárkányi F, Takács S, Ditrói F, Baba M, Ohtshuki T, Spahn I, Ignatyuk AV (2009) Excitation functions for production of medically relevant radioisotopes in deuteron irradiations of Pr and Tm targets. Nucl Instrum Methods Phys Res B 267:727-736. EXFOR: D4209

326. Hermanne A, Tárkányi F, Takács S, Ditrói F (2016) Extension of excitation functions up to $50 \mathrm{MeV}$ for activation products in deuteron irradiations of Pr and Tm targets. Nucl Instrum Methods Phys Res B 383:81-88. EXFOR: D4355

327. Nichols AL, Qaim SM, Capote Noy R (2011) Summary report of a technical meeting on intermediate-term nuclear data needs for medical applications: cross sections and decay data, 22-26 August 2011. IAEA Headquarters, IAEA report INDC(NDS)-0596, September 2011, IAEA, Vienna, Austria. Available online at: https://www-nds.iaea.org/publications/indc/ indc-nds-0596.pdf

328. Qaim SM (2017) Nuclear data for production and medical application of radionuclides: present status and future needs. Nucl Med Biol 44:31-49

329. Capote Noy R, Nichols AL (2008) Summary report of a consultants' meeting on high-precision beta-intensity measurements and evaluations for specific PET radioisotopes, 3-5 September 2008. IAEA Headquarters, IAEA report INDC(NDS)-0535, December 2008, IAEA, Vienna, Austria. Available online at: www-nds.iaea. org/publications/indc/indc-nds-0535.pdf 